\title{
2016 ESC Guidelines for the diagnosis and treatment of acute and chronic heart failure
}

\section{The Task Force for the diagnosis and treatment of acute and chronic heart failure of the European Society of Cardiology (ESC)}

\section{Developed with the special contribution of the Heart Failure Association (HFA) of the ESC}

\begin{abstract}
Authors/Task Force Members: Piotr Ponikowski* (Chairperson) (Poland), Adriaan A. Voors* (Co-Chairperson) (The Netherlands), Stefan D. Anker (Germany), Héctor Bueno (Spain), John G. F. Cleland (UK), Andrew J. S. Coats (UK), Volkmar Falk (Germany), José Ramón González-Juanatey (Spain), Veli-Pekka Harjola (Finland), Ewa A. Jankowska (Poland), Mariell Jessup (USA), Cecilia Linde (Sweden), Petros Nihoyannopoulos (UK), John T. Parissis (Greece), Burkert Pieske (Germany), Jillian P. Riley (UK), Giuseppe M. C. Rosano (UK/Italy), Luis M. Ruilope (Spain), Frank Ruschitzka (Switzerland), Frans H. Rutten (The Netherlands), Peter van der Meer (The Netherlands)
\end{abstract}

Document Reviewers: Gerasimos Filippatos (CPG Review Coordinator) (Greece), John J. V. McMurray (CPG Review Coordinator) (UK), Victor Aboyans (France), Stephan Achenbach (Germany), Stefan Agewall (Norway), Nawwar Al-Attar (UK), John James Atherton (Australia), Johann Bauersachs (Germany), A. John Camm (UK), Scipione Carerj (Italy), Claudio Ceconi (Italy), Antonio Coca (Spain), Perry Elliott (UK), Çetin Erol (Turkey), Justin Ezekowitz (Canada), Covadonga Fernández-Golfín (Spain), Donna Fitzsimons (UK), Marco Guazzi (Italy),

* Corresponding authors: Piotr Ponikowski, Department of Heart Diseases, Wroclaw Medical University, Centre for Heart Diseases, Military Hospital, ul. Weigla 5, 50-981 Wroclaw, Poland, Tel: +48 261660 279, Tel/Fax: +48 261660 237, E-mail: piotrponikowski@4wsk.pl.

Adriaan Voors, Cardiology, University of Groningen, University Medical Center Groningen, Hanzeplein 1, PO Box $30.001,9700$ RB Groningen, The Netherlands, Tel: +31 50 3612355 , Fax: +3150 3614391, E-mail: a.a.voors@umcg.nl.

ESC Committee for Practice Guidelines (CPG) and National Cardiac Societies document reviewers: listed in the Appendix.

ESC entities having participated in the development of this document:

Associations: Acute Cardiovascular Care Association (ACCA), European Association for Cardiovascular Prevention and Rehabilitation (EACPR), European Association of Cardiovascular Imaging (EACVI), European Heart Rhythm Association (EHRA), Heart Failure Association (HFA).

Councils: Council on Cardiovascular Nursing and Allied Professions, Council for Cardiology Practice, Council on Cardiovascular Primary Care, Council on Hypertension.

Working Groups: Cardiovascular Pharmacotherapy, Cardiovascular Surgery, Myocardial and Pericardial Diseases, Myocardial Function, Pulmonary Circulation and Right Ventricular Function, Valvular Heart Disease.

The content of these European Society of Cardiology (ESC) Guidelines has been published for personal and educational use only. No commercial use is authorized. No part of the ESC Guidelines may be translated or reproduced in any form without written permission from the ESC. Permission can be obtained upon submission of a written request to John Wiley \& Sons, the publisher of the European Journal of Heart Failure and the party authorized to handle such permissions on behalf of the ESC (permissions@wiley.com).

Disclaimer. The ESC Guidelines represent the views of the ESC and were produced after careful consideration of the scientific and medical knowledge and the evidence available at the time of their publication. The ESC is not responsible in the event of any contradiction, discrepancy and/or ambiguity between the ESC Guidelines and any other official recommendations or guidelines issued by the relevant public health authorities, in particular in relation to good use of healthcare or therapeutic strategies. Health professionals are encouraged to take the ESC Guidelines fully into account when exercising their clinical judgment, as well as in the determination and the implementation of preventive, diagnostic or therapeutic medical strategies; however, the ESC Guidelines do not override, in any way whatsoever, the individual responsibility of health professionals to make appropriate and accurate decisions in consideration of each patient's health condition and in consultation with that patient and, where appropriate and/or necessary, the patient's caregiver. Nor do the ESC Guidelines exempt health professionals from taking into full and careful consideration the relevant official updated recommendations or guidelines issued by the competent public health authorities, in order to manage each patient's case in light of the scientifically accepted data pursuant to their respective ethical and professional obligations. It is also the health professional's responsibility to verify the applicable rules and regulations relating to drugs and medical devices at the time of prescription.

The article has been co-published with permission in European Heart Journal and European Journal of Heart Failure. All rights reserved in respect of European Heart Journal. (C) European Society of Cardiology 2016. All rights reserved. For permissions please email: permissions@wiley.com. 
Maxime Guenoun (France), Gerd Hasenfuss (Germany), Gerhard Hindricks (Germany), Arno W. Hoes (The Netherlands), Bernard lung (France), Tiny Jaarsma (Sweden), Paulus Kirchhof (UK/Germany), Juhani Knuuti (Finland), Philippe Kolh (Belgium), Stavros Konstantinides (Germany/Greece), Mitja Lainscak (Slovenia), Patrizio Lancellotti (Belgium), Gregory Y. H. Lip (UK), Francesco Maisano (Switzerland), Christian Mueller (Switzerland), Mark C. Petrie (UK), Massimo F. Piepoli (Italy), Silvia G. Priori (Italy), Adam Torbicki (Poland), Hiroyuki Tsutsui (Japan), Dirk J. van Veldhuisen (The Netherlands), Stephan Windecker (Switzerland), Clyde Yancy (USA), Jose Luis Zamorano (Spain)

The disclosure forms of all experts involved in the development of these guidelines are available on the ESC website http://www.escardio.org/guidelines.

Keywords

Guidelines - Heart failure $\bullet$ Natriuretic peptides • Ejection fraction • Diagnosis • Pharmacotherapy • Neuro-hormonal antagonists • Cardiac resynchronization therapy • Mechanical circulatory support • Transplantation • Arrhythmias • Co-morbidities • Hospitalization • Multidisciplinary management

\section{Table of Contents}

Abbreviations and acronyms ................ 893

1. Preamble . . . . . . . . . . . . . . . . . . . . . . 896

2. Introduction . . . . . . . . . . . . . . . . . . . . 898

3. Definition, epidemiology and prognosis . . . . . . . . 898

3.1 Definition of heart failure . . . . . . . . . . . . . . . 898

3.2 Terminology . . . . . . . . . . . . . . . . . . . . . . 899

3.2.1 Heart failure with preserved, mid-range and reduced ejection fraction . . . . . . . . . . . . . . . . . . . . 899 3.2.2 Terminology related to the time course of heart

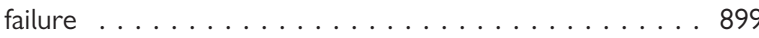
3.2.3 Terminology related to the symptomatic severity of heart failure . . . . . . . . . . . . . . . . . . 900

3.3 Epidemiology, aetiology and natural history of heart failure 900

3.4 Prognosis . . . . . . . . . . . . . . . . . . . . . . . . . . . . . . . . . . 900

4. Diagnosis . . . . . . . . . . . . . . . . . . . . . . 900

4.1 Symptoms and signs . . . . . . . . . . . . . . . . 900

4.2 Essential initial investigations: natriuretic peptides,

electrocardiogram, and echocardiography . . . . . . . . . . . 901

4.3 Algorithm for the diagnosis of heart failure . . . . . . . 902

4.3.1 Algorithm for the diagnosis of heart failure in the non-acute setting . . . . . . . . . . . . . 902

4.3.2 Diagnosis of heart failure with preserved ejection fraction . . . . . . . . . . . . . . . . . . . . . 902

5. Cardiac imaging and other diagnostic tests . . . . . . . . . 904

5.1 Chest X-ray . . . . . . . . . . . . . . . . . . . . . . . 904

5.2 Transthoracic echocardiography ... . . . . . . . . . . . 904

5.2.1 Assessment of left ventricular systolic function . . . . 904

5.2.2 Assessment of left ventricular diastolic function . . . 905

5.2.3 Assessment of right ventricular function and

pulmonary arterial pressure . . . . . . . . . . . . . . 905

5.3 Transoesophageal echocardiography . . . . . . . . . 905

5.4 Stress echocardiography . . . . . . . . . . . . . . . . 905

5.5 Cardiac magnetic resonance . . . . . . . . . . . . . . 905

5.6 Single-photon emission computed tomography and radionuclide ventriculography . . . . . . . . . . . . . . . 905

5.7 Positron emission tomography . . . . . . . . . . . . . 905

5.8 Coronary angiography . . . . . . . . . . . . . . . . . 906
5.9 Cardiac computed tomography . . . . . . . . . . . . . . 906

5.10 Other diagnostic tests . . . . . . . . . . . . . . . . 907

5.10.1 Genetic testing in heart failure . . . . . . . . . . 907

6. Delaying or preventing the development of overt heart

failure or preventing death before the onset of symptoms . . . . 908

7. Pharmacological treatment of heart failure with reduced ejection fraction . . . . . . . . . . . . . . . . . . . . . 909

7.1 Objectives in the management of heart failure . . . . . 909

7.2 Treatments recommended in all symptomatic patients

with heart failure with reduced ejection fraction . . . . . . . . 910

7.2.1 Angiotensin-converting enzyme inhibitors . . . . . . 910

7.2 .2 Beta-blockers. . . . . . . . . . . . . . . . . . 910

7.2.3 Mineralocorticoid/aldosterone receptor antagonists . 910

7.3 Other treatments recommended in selected symptomatic patients with heart failure with reduced ejection fraction . . 910

7.3 .1 Diuretics . . . . . . . . . . . . . . . . . . . . . . . . 910

7.3.2 Angiotensin receptor neprilysin inhibitor . . . . . . . 913

$7.3 .3 I_{\mathrm{f}}$-channel inhibitor . . . . . . . . . . . . . . 914

7.3.4 Angiotensin II type I receptor blockers . . . . . . . . 914

7.3.5 Combination of hydralazine and isosorbide

dinitrate . . . . . . . . . . . . . . . . . . . . . . . . . 914

7.4 Other treatments with less certain benefits in

symptomatic patients with heart failure with reduced ejection fraction . . . . . . . . . . . . . . . . . . . . . . 914

7.4.1 Digoxin and other digitalis glycosides . . . . . . . . 914

7.4 .2 n-3 polyunsaturated fatty acids . . . . . . . . . . 915

7.5 Treatments not recommended (unproven benefit) in

symptomatic patients with heart failure with reduced ejection fraction . . . . . . . . . . . . . . . . . . 915

7.5.1 3-Hydroxy-3-methylglutaryl-coenzyme A reductase inhibitors ('statins') . . . . . . . . . . . . . . . . . 915

7.5.2 Oral anticoagulants and antiplatelet therapy . . . . . 915

7.5 .3 Renin inhibitors . . . . . . . . . . . . . . . 915

7.6 Treatments not recommended (believed to cause harm) in symptomatic patients with heart failure with reduced ejection fraction. . . . . . . . . . . . . . . . . . 916

7.6.1 Calcium-channel blockers . . . . . . . . . . . . 916 
8. Non-surgical device treatment of heart failure with reduced ejection fraction . . . . . . . . . . . . . 916

8.1 Implantable cardioverter-defibrillator . . . . . . . . . . 916

8.1.1 Secondary prevention of sudden cardiac death . . . . 916

8.1.2 Primary prevention of sudden cardiac death . . . . . 917

8.2 Cardiac resynchronization therapy . . . . . . . . . . . 918

8.3 Other implantable electrical devices . . . . . . . . . . . 919

9. Treatment of heart failure with preserved ejection fraction . . 919

9.1 Effect of treatment on symptoms in heart failure with

preserved ejection fraction. . . . . . . . . . . 920

9.2 Effect of treatment on hospitalization for heart failure in

heart failure with preserved ejection fraction . . . . . . . 920

9.3 Effect of treatment on mortality in heart failure with

preserved ejection fraction. . . . . . . . . . . . . . 920

9.4 Other considerations . . . . . . . . . . . . . . . . 920

10. Arrhythmias and conductance disturbances . . . . . . . . 920

10.1 Atrial fibrillation . . . . . . . . . . . . . . . . 921

10.1.1 Prevention of atrial fibrillation in patients with heart

failure . . . . . . . . . . . . . . . . 921

10.1.2 Management of new-onset, rapid atrial fibrillation in

patients with heart failure . . . . . . . . . . . . . . . . 921

10.1.3 Rate control . . . . . . . . . . . . . . . . . 921

10.1.4 Rhythm control . . . . . . . . . . . . . . . . 922

10.1.5 Thromboembolism prophylaxis . . . . . . . . . 923

10.2 Ventricular arrhythmias . . . . . . . . . . . . . . 923

10.3 Symptomatic bradycardia, pauses and

atrio-ventricular block . . . . . . . . . . . . . . . . . . . . 924

11. Co-morbidities . . . . . . . . . . . . . . . 925

11.1 Heart failure and co-morbidities . . . . . . . . 925

11.2 Angina and coronary artery disease . . . . . . . . . 925

11.2.1 Pharmacological management . . . . . . . . . . . 925

11.2.2 Myocardial revascularization . . . . . . . . . . 925

11.3 Cachexia and sarcopenia (for frailty, please refer to

Section 14) . . . . . . . . . . . . . . . . 926

11.4 Cancer . . . . . . . . . . . . . . . . . 926

11.5 Central nervous system (including depression, stroke and

autonomic dysfunction) . . . . . . . . . . . . . . 927

11.6 Diabetes . . . . . . . . . . . . . . . . . . . . . . . . . . . . . . . . . . . .

11.7 Erectile dysfunction . . . . . . . . . . . . . . . . . . . . . . . . . . . . 928

11.8 Gout and arthritis . . . . . . . . . . . . . . . . 928

11.9 Hypokalaemia and hyperkalaemia . . . . . . . . . . . . . . . . . 928

11.10 Hyperlipidaemia . . . . . . . . . . . . . . . . 928

11.11 Hypertension . . . . . . . . . . . . . . . . . 928

11.12 Iron deficiency and anaemia . . . . . . . . . . . . 929

11.13 Kidney dysfunction (including chronic kidney disease,

acute kidney injury, cardio-renal syndrome, and prostatic

obstruction) . . . . . . . . . . . . . . . . . 930

11.14 Lung disease (including asthma and chronic obstructive

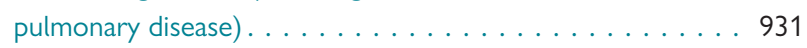

11.15 Obesity. . . . . . . . . . . . . . . . . . 931

11.16 Sleep disturbance and sleep-disordered

breathing . . . . . . . . . . . . . . . . . . 931

11.17 Valvular heart disease. . . . . . . . . . . . . . 932

11.17.1 Aortic stenosis . . . . . . . . . . . . . . . . 932

11.17.2 Aortic regurgitation . . . . . . . . . . . . . . . . . . . . . . . . . . .

11.17.3 Mitral regurgitation . . . . . . . . . . . . . . 932

11.17.4 Tricuspid regurgitation . . . . . . . . . . . . 932

12. Acute heart failure . . . . . . . . . . . . . . . . . . 933

12.1 Definition and classification . . . . . . . . . . . . 933

12.2 Diagnosis and initial prognostic evaluation . . . . . . . 934

12.3 Management . . . . . . . . . . . . . . . . . 938

12.3.1 Identification of precipitants/causes leading to decompensation that needs urgent management . . . . . 938 12.3.2 Criteria for hospitalization in ward vs intensive care/coronary care unit . . . . . . . . . . . . . . . . 939

12.3.3 Management of the early phase . . . . . . . . . 939

12.3.4 Management of patients with cardiogenic shock . . 944

12.4 Management of evidence-based oral therapies . . . . . 944

12.5 Monitoring of clinical status of patients hospitalized due

to acute heart failure . . . . . . . . . . . . . 945

12.6 Criteria for discharge from hospital and follow-up in

high-risk period . . . . . . . . . . . . . . . . . . . . 945

12.7 Goals of treatment during the different stages of

management of acute heart failure . . . . . . . . . . . . 945

13. Mechanical circulatory support and heart transplantation . . 946

13.1 Mechanical circulatory support . . . . . . . . . . . 946

13.1.1 Mechanical circulatory support in acute heart

failure . . . . . . . . . . . . . . . . 946

13.1.2 Mechanical circulatory support in end-stage chronic

heart failure . . . . . . . . . . . . . . . . . . . . 946

13.2 Heart transplantation. . . . . . . . . . . . . . . . . 948

14. Multidisciplinary team management . . . . . . . . . . . . . . 949

14.1 Organization of care . . . . . . . . . . . . . . . . . 949

14.2 Discharge planning . . . . . . . . . . . . . . . . 951

14.3 Lifestyle advice . . . . . . . . . . . . . . . . . . 951

14.4 Exercise training . . . . . . . . . . . . . . . . 951

14.5 Follow-up and monitoring . . . . . . . . . . . . . 951

14.6 The older adult, frailty and cognitive impairment . . . . 952

14.7 Palliative and end-of-life care . . . . . . . . . . . . . . . . . . . . . . . . . . . . .

15. Gaps in evidence . . . . . . . . . . . . . . . . . . 953

16. To do and not to do messages from the Guidelines. . . . . . 954

17. Web Addenda . . . . . . . . . . . . . . . . . . . . 955

18. Appendix . . . . . . . . . . . . . . . . . . 956

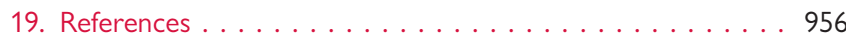

\section{Abbreviations and acronyms}

\section{ACC/AHA}

ACCF/AHA

ACE

ACEI

ACS

AF

AHF

AHI

AIDS

AKI

Aldo-DHF

$A L$

ALT
American College of Cardiology/American Heart Association

American College of Cardiology Foundation/ American Heart Association

angiotensin-converting enzyme

angiotensin-converting enzyme inhibitor

acute coronary syndrome

atrial fibrillation

acute heart failure

apnoea/hypopnoea index

acquired immunodeficiency syndrome

acute kidney injury

aldosterone receptor blockade in diastolic heart failure

amyloid light chain

alanine aminotransferase 
AMI

AMICA

ANP

ANS

ARB

ARNI

ARVC

AST

ASV

ATLAS

ATTR

AV

AVP

b.i.d.

BioPACE

BiPAP

BiVAD

BLOCK-HF

BMI

BNP

BP

bpm

BSA

BTB

BTC

BTD

BTR

BTT

BUN

CABANA

CABG

CAD

CARE-HF

CASTLE-AF

CCB

CCM

CCS

$\mathrm{CCU}$

$\mathrm{CHA}_{2} \mathrm{DS}_{2}$-VASc acute myocardial infarction

Atrial fibrillation Management In Congestive heart failure with Ablation

A-type natriuretic peptide

autonomic nervous system

angiotensin receptor blocker

angiotensin receptor neprilysin inhibitor

arrhythmogenic right ventricular

cardiomyopathy

aspartate aminotransferase

assisted servo-ventilation

Assessment of Treatment with Lisinopril And

Survival

transthyretin-mediated amyloidosis

atrio-ventricular

arginine vasopressin

bis in die (twice daily)

Biventricular Pacing for Atrio-ventricular

Block to Prevent Cardiac Desynchronization

bilevel positive airway pressure

biventricular assist device

Biventricular versus Right Ventricular Pacing in

Heart Failure Patients with Atrio-ventricular

Block

body mass index

B-type natriuretic peptide

blood pressure

beats per minute

body surface area

bridge to bridge

bridge to candidacy

bridge to decision

bridge to recovery

bridge to transplantation

blood urea nitrogen

Catheter ABlation versus ANtiarrhythmic drug therapy for Atrial fibrillation

coronary artery bypass graft/grafting

coronary artery disease

CArdiac REsynchronization in Heart Failure

Catheter Ablation versus Standard conven-

tional Treatment in patients with LEft ven-

tricular dysfunction and Atrial Fibrillation

calcium-channel blocker

cardiac contractility modulation

Canadian Cardiovascular Society

coronary care unit

Congestive heart failure or left ventricular dys-

function, Hypertension, Age $\geq 75$ (doubled),

Diabetes, Stroke (doubled)-Vascular disease,

Age 65-74, Sex category (female)

CHARM-Alternative Candesartan in heart failure assessment of reduction in mortality and morbidity

CHARM-Added
Candesartan Cilexetil in Heart Failure Assessment of Reduction in Mortality and Morbidity
CHARM-Preserved

Candesartan Cilexetil in Heart Failure Assessment of Reduction in Mortality and Morbidity cardiac index

CI-AKI
CIBIS II
CK
CKD
CK-MB
CMP
CMR
COMPANION

CONFIRM-HF

CONSENSUS

COPD

COPERNICUS

COX-2 inhibitor

CPAP

CPG

CRT

CRT-D

CRT-P

CSA

CSR

CT

CYP3A4

DCM

DES

DHA

DIG-PEF

DNA

DOSE

DPD

DPP4i

DT

$\mathrm{e}^{\prime}$

ECG

Echo-CRT

ECLS

ECMO

ED

EF

eGFR

EHRA

EMA

EMB

EMF contrast-induced acute kidney injury

Cardiac Insufficiency Bisoprolol Study II

creatine kinase

chronic kidney disease

creatine kinase MB

cardiomyopathy

cardiac magnetic resonance

Comparison of Medical Therapy, Pacing, and

Defibrillation in Heart Failure

Ferric CarboxymaltOse evaluatioN on per-

Formance in patients with IRon deficiency

in coMbination with chronic Heart Failure

Cooperative North Scandinavian Enalapril

Survival Study

chronic obstructive pulmonary disease

Carvedilol Prospective Randomized Cumulative Survival

cyclooxygenase-2 inhibitor

continuous positive airway pressure

Committee for Practice Guidelines

cardiac resynchronization therapy

defibrillator with cardiac resynchronization

therapy

pacemaker with cardiac resynchronization

therapy

central sleep apnoea

Cheyne-Stokes respiration

computed tomography

cytochrome P450 3A4

dilated cardiomyopathy

desmin

docosahexaenoic acid

ancillary Digitalis Investigation Group trial

deoxyribonucleic acid

Diuretic Optimization Strategies Evaluation

3,3-diphosphono-1,2-propanodicarboxylic acid

dipeptidyl peptidase-4 inhibitor

destination therapy

early diastolic tissue velocity

electrocardiogram

Echocardiography Guided Cardiac Resynchronization Therapy

extracorporeal life support

extracorporeal membrane oxygenation

emergency department

ejection fraction

estimated glomerular filtration rate

European Heart Rhythm Association

European Medicines Agency

endomyocardial biopsy

endomyocardial fibrosis 
EMPHASIS-HF

EPA

EPHESUS

ESC

EU

EULAR

Ex-DHF

FACIT-Pal

FAIR-HF

FCM

$\mathrm{FiO}_{2}$

GFR

GGTP

$\mathrm{GH}$

GLS

GLP-1

HAS-BLED

HbA1c
HCM
HES
HF
HFA
HFmrEF
HFpEF
HFrEF
H-ISDN
HIVIAIDS
HR
Hs troponin
IABP
IABP-SHOCK
IABP-SHOCK II
ICD
ICU
IHD
IL
INH
INTERMACS
IN-TIME
IPD
I-PRESERVE
i.v.
IVC

Eplerenone in Mild Patients Hospitalization and Survival Study in Heart Failure

eicosapentaenoic acid

Eplerenone Post-Acute Myocardial Infarction Heart Failure Efficacy and Survival Study

European Society of Cardiology

European Union

European League Against Rheumatism

Exercise training in Diastolic Heart Failure

Functional Assessment of Chronic Illness

Therapy - Palliative Care

Ferinject Assessment in Patients with Iron

Deficiency and Chronic Heart Failure

ferric carboxymaltose

fraction of inspired oxygen

glomerular filtration rate

gamma-glutamyl transpeptidase

growth hormone

global longitudinal strain

glucagon-like peptide 1

Hypertension, Abnormal renal/liver function (1 point each), Stroke, Bleeding history or predisposition, Labile international normalized ratio, Elderly ( $>65$ years), Drugs/alcohol concomitantly (1 point each)

glycated haemoglobin

hypertrophic cardiomyopathy

hypereosinophilic syndrome

heart failure

Heart Failure Association

heart failure with mid-range ejection fraction

heart failure with preserved ejection fraction

heart failure with reduced ejection fraction

hydralazine and isosorbide dinitrate

human immunodeficiency virus/acquired

immune deficiency syndrome

heart rate

high sensitivity troponin

intra-aortic balloon pump

IntraAortic Balloon Pump in Cardiogenic Shock

IntraAortic Balloon Pump in Cardiogenic

Shock II

implantable cardioverter-defibrillator

intensive care unit

ischaemic heart disease

interleukin

Interdisciplinary Network for Heart Failure

Interagency Registry for Mechanically

Assisted Circulatory Support

Implant-based multiparameter telemonitor-

ing of patients with heart failure

individual patient data

Irbesartan in Heart Failure with Preserved

Ejection Fraction Study

intravenous

inferior vena cava
IVRT

KCCQ

LA

LAE

LAVI

LBBB

LGE

LMNA

LMWH

LV

LVAD

LVEDP

LVEDV

LVEF

LVESV

LVID

LVMI

LVSD

MADIT-CRT

MCS

MERIT-HF

MR

MRA

MR-proANP

MV

MV A-Wave

MV E-Wave

MYBPC3

MYH7

n-3 PUFA

NEP

NOAC

$N P$

NPPV

NSAID

NSTE-ACS

NT-proBNP

NYHA

o.d.

OMT

OSA

$\mathrm{PaCO}_{2}$

$\mathrm{PAH}$

$\mathrm{PaO}_{2}$

PARADIGM-HF

PARAMOUNT

$\mathrm{PCl}$ isovolumetric relaxation time

Kansas City Cardiomyopathy Questionnaire

left atrial/atrium

left atrial enlargement

left atrial volume index

left bundle branch block

late gadolinium enhancement

lamin A/C

low-molecular-weight heparin

left ventricular/left ventricle

left ventricular assist device

left ventricular end diastolic pressure

left ventricular end diastolic volume

left ventricular ejection fraction

left ventricular end systolic volume

left ventricular internal dimension

left ventricular mass index

left ventricular systolic dysfunction

Multicenter Automatic Defibrillator Implantation Trial with Cardiac Resynchronization

Therapy

mechanical circulatory support

Metoprolol CR/XL Randomised Intervention

Trial in Congestive Heart Failure

mineralocorticoid receptor/magnetic

resonance

mineralocorticoid receptor antagonist

mid-regional pro A-type natriuretic peptide

mitral valve

mitral valve late diastolic flow

mitral valve early diastolic flow

cardiac myosin binding protein $C$

cardiac $\beta$-myosin heavy chain

n-3 polyunsaturated fatty acid

neprilysin

non-vitamin $\mathrm{K}$ antagonist oral anticoagulant

natriuretic peptide

non-invasive positive pressure ventilation

non-steroidal anti-inflammatory drug

non-ST elevation acute coronary syndrome

$\mathrm{N}$-terminal pro-B type natriuretic peptide

New York Heart Association

omne in die (once daily)

optimal medical therapy

obstructive sleep apnoea

partial pressure of carbon dioxide in arterial blood

pulmonary arterial hypertension

partial pressure of oxygen in arterial blood

Prospective Comparison of ARNI with ACEI

to Determine Impact on Global Mortality and

Morbidity in Heart Failure Trial

LCZ696 Compared to Valsartan in Patients

With Chronic Heart Failure and Preserved

Left-ventricular Ejection Fraction

percutaneous coronary intervention 
PCWP

PDE5I

Peak $\mathrm{VO}_{2}$

PEP-CHF

PET

PLN

PPV

PRISMA 7

PROTECT ॥

PS-PEEP

PV

PVR

QALY

QRS

RA

RAAS

RAFT

RALES

RCT

RELAX

REVERSE

RV

RVAD

SADHART

SAVE

SBP

SCD-HeFT

SDB

SENIORS

SERVE-HF

SHIFT

SIGNIFY

SOLVD

SPECT pulmonary capillary wedge pressure

phosphodiesterase 5 inhibitor

peak oxygen uptake

Perindopril in Elderly People with Chronic Heart Failure

positron emission tomography

phospholamban

positive pressure ventilation

seven-item, self-completion questionnaire to identify older adults with moderate to severe disabilities

Prospective, Multi-center, Randomized Controlled Trial of the IMPELLA RECOVER

LP 2.5 System Versus Intra Aortic Balloon Pump (IABP) in Patients Undergoing Non Emergent High Risk PCI

pressure-support positive end-expiratory pressure

pulmonary vein

pulmonary vascular resistance

quality-adjusted life year

$\mathrm{Q}, \mathrm{R}$, and $\mathrm{S}$ waves (combination of three of the graphical deflections)

right atrium/atrial

renin-angiotensin-aldosterone system

Resynchronization-Defibrillation for Ambulatory Heart Failure Trial

Randomized Aldactone Evaluation Study

randomized controlled trial

Phosphodiesterase-5 Inhibition to Improve

Clinical Status and Exercise Capacity in

Diastolic Heart Failure

REsynchronization reVErses Remodeling in Systolic left vEntricular dysfunction

right ventricular/ventricle

right ventricular assist device

Sertraline Antidepressant Heart Attack

Randomized Trial

Survival After Veno-arterial ECMO

systolic blood pressure

Sudden Cardiac Death in Heart Failure Trial sleep-disordered breathing

Study of the Effects of Nebivolol Intervention on Outcomes and Rehospitalisations in Seniors with Heart Failure

Treatment of sleep-disordered breathing with predominant central sleep apnoea with adaptive Servo-ventilation in patients with chronic heart failure

Systolic Heart failure treatment with the $I_{f}$ inhibitor ivabradine Trial

Study Assessing the Morbidity-Mortality Benefits of the $I_{f}$ Inhibitor Ivabradine in Patients with Coronary Artery Disease Studies of Left Ventricular Dysfunction single-photon emission computed tomography

\begin{tabular}{|c|c|}
\hline $\mathrm{SpO}_{2}$ & transcutaneous oxygen saturation \\
\hline SPPB & Short Physical Performance Battery \\
\hline SPRINT & $\begin{array}{l}\text { Systolic Blood Pressure Intervention } \\
\text { Trial }\end{array}$ \\
\hline STEMI & $\begin{array}{l}\text { ST segment elevation myocardial } \\
\text { infarction }\end{array}$ \\
\hline $\mathrm{STICH}$ & $\begin{array}{l}\text { Surgical Treatment for Ischemic Heart } \\
\text { Failure }\end{array}$ \\
\hline STS & structured telephone support \\
\hline TAPSE & tricuspid annular plane systolic excursion \\
\hline TAVI & transaortic valve implantation \\
\hline TDI & tissue Doppler imaging \\
\hline TECOS & $\begin{array}{l}\text { Trial Evaluating Cardiovascular Outcomes } \\
\text { with Sitagliptin }\end{array}$ \\
\hline TEHAF & $\begin{array}{l}\text { Telemonitoring in Patients with Heart } \\
\text { Failure }\end{array}$ \\
\hline Tele-HF & $\begin{array}{l}\text { Telemonitoring to Improve Heart } \\
\text { Failure Outcomes }\end{array}$ \\
\hline TIA & transient ischaemic attack \\
\hline TIBC & total iron-binding capacity \\
\hline t.i.d. & ter in die (three times a day) \\
\hline TIM-HF & $\begin{array}{l}\text { Telemedical Interventional Monitoring in } \\
\text { Heart Failure }\end{array}$ \\
\hline TOE & transoesophageal echocardiography \\
\hline TOPCAT & $\begin{array}{l}\text { Treatment of Preserved Cardiac Function } \\
\text { Heart Failure with an Aldosterone } \\
\text { Antagonist }\end{array}$ \\
\hline TR & tricuspid regurgitation \\
\hline TRV & tricuspid regurgitation velocity \\
\hline TSAT & transferrin saturation \\
\hline $\mathrm{TSH}$ & thyroid-stimulating hormone \\
\hline TTE & transthoracic echocardiography \\
\hline TTN & titin \\
\hline ULT & urate lowering therapy \\
\hline VAD & ventricular assist device \\
\hline Val-HeFT & Valsartan Heart Failure Trial \\
\hline VE-VCO 2 & $\begin{array}{l}\text { ventilatory equivalent ratio for carbon } \\
\text { dioxide }\end{array}$ \\
\hline VT & ventricular tachycardia \\
\hline VV interval & interventricular pacing interval \\
\hline WBC & white blood cells \\
\hline WISH & $\begin{array}{l}\text { Weight Monitoring in Patients with Severe } \\
\text { Heart Failure }\end{array}$ \\
\hline WRF & worsening renal function \\
\hline
\end{tabular}

\section{Preamble}

Guidelines summarize and evaluate all available evidence on a particular issue at the time of the writing process, with the aim of assisting health professionals in selecting the best management strategies for an individual patient with a given condition, taking into account the impact on outcome, as well as the riskbenefit ratio of particular diagnostic or therapeutic means. Guidelines and recommendations should help health professionals to make decisions in their daily practice. However, the final decisions concerning an individual patient must be made by the responsible health professional(s) in consultation with the patient and caregiver as appropriate. 
A great number of Guidelines have been issued in recent years by the European Society of Cardiology (ESC) as well as by other societies and organisations. Because of the impact on clinical practice, quality criteria for the development of guidelines have been established in order to make all decisions transparent to the user. The recommendations for formulating and issuing ESC Guidelines can be found on the ESC website (http://www.escardio.org/Guidelines\&-Education/Clinical-Practice-Guidelines/Guidelines-development/ Writing-ESC-Guidelines). ESC Guidelines represent the official position of the ESC on a given topic and are regularly updated.

Members of this Task Force were selected by the ESC to represent professionals involved with the medical care of patients with this pathology. Selected experts in the field undertook a comprehensive review of the published evidence for management (including diagnosis, treatment, prevention and rehabilitation) of a given condition according to ESC Committee for Practice Guidelines (CPG) policy. A critical evaluation of diagnostic and therapeutic procedures was performed, including assessment of the risk-benefit ratio. Estimates of expected health outcomes for larger populations were included, where data exist. The level of evidence and the strength of the recommendation of particular management options were weighed and graded according to predefined scales, as outlined in Tables 1.1 and 1.2.

The experts of the writing and reviewing panels provided declarations of interest forms for all relationships that might be perceived as real or potential sources of conflicts of interest. These forms were compiled into one file and can be found on the ESC website (http:// www.escardio.org/guidelines). Any changes in declarations of interest that arise during the writing period must be notified to the ESC and updated. The Task Force received its entire financial support from the ESC without any involvement from the healthcare industry.

The ESC CPG supervises and coordinates the preparation of new Guidelines produced by task forces, expert groups or consensus panels. The Committee is also responsible for the endorsement process of these Guidelines. The ESC Guidelines undergo extensive review by the CPG and external experts. After appropriate revisions the Guidelines are approved by all the experts involved in the Task Force. The finalized document is approved by the CPG for publication in the European Heart Journal. The Guidelines were developed after careful consideration of the scientific and medical knowledge and the evidence available at the time of their dating.

The task of developing ESC Guidelines covers not only integration of the most recent research, but also the creation of educational tools and implementation programmes for the recommendations. To implement the guidelines, condensed pocket guidelines versions, summary slides, booklets with essential messages, summary cards for non-specialists, and an electronic version for digital applications (smartphones, etc.) are produced. These versions are abridged and thus, if needed, one should always refer to the full text version, which is freely available on the ESC website. The National Cardiac Societies of the ESC are encouraged to endorse, translate and implement all ESC Guidelines. Implementation programmes are needed because

\section{Table I.2 Level of evidence}

\begin{tabular}{|l|l|}
\hline $\begin{array}{l}\text { Level of } \\
\text { evidence A }\end{array}$ & $\begin{array}{l}\text { Data derived from multiple randomized } \\
\text { clinical trials or meta-analyses. }\end{array}$ \\
\hline $\begin{array}{l}\text { Level of } \\
\text { evidence B }\end{array}$ & $\begin{array}{l}\text { Data derived from a single randomized } \\
\text { clinical trial or large non-randomized } \\
\text { studies. }\end{array}$ \\
\hline Level of \\
evidence C & $\begin{array}{l}\text { Consensus of opinion of the experts and/ } \\
\text { or small studies, retrospective studies, } \\
\text { registries. }\end{array}$ \\
\hline
\end{tabular}

Table I.I Classes of recommendations

\begin{tabular}{|c|c|c|}
\hline $\begin{array}{l}\text { Classes of } \\
\text { recommendations }\end{array}$ & Definition & $\begin{array}{c}\text { Suggested wording to } \\
\text { use }\end{array}$ \\
\hline Class I & $\begin{array}{l}\text { Evidence and/or general agreement } \\
\text { that a given treatment or } \\
\text { procedure is beneficial, useful, } \\
\text { effective. }\end{array}$ & $\begin{array}{l}\text { Is recommended/is } \\
\text { indicated }\end{array}$ \\
\hline Class II & $\begin{array}{l}\text { Conflicting evidence and/or a } \\
\text { divergence of opinion about the } \\
\text { usefulness/efficacy of the given } \\
\text { treatment or procedure. }\end{array}$ & \\
\hline Class IIa & $\begin{array}{l}\text { Weight of evidence/opinion is in } \\
\text { favour of usefulness/efficacy. }\end{array}$ & Should be considered \\
\hline Class IIb & $\begin{array}{l}\text { Usefulness/efficacy is less well } \\
\text { established by evidence/opinion. }\end{array}$ & May be considered \\
\hline Class III & $\begin{array}{l}\text { Evidence or general agreement that } \\
\text { the given treatment or procedure } \\
\text { is not useful/effective; and in some } \\
\text { cases may be harmful. }\end{array}$ & Is not recommended \\
\hline
\end{tabular}


it has been shown that the outcome of disease may be favourably influenced by the thorough application of clinical recommendations.

Surveys and registries are needed to verify that real-life daily practice is in keeping with what is recommended in the guidelines, thus completing the loop between clinical research, writing of guidelines, disseminating them and implementing them into clinical practice.

Health professionals are encouraged to take the ESC Guidelines fully into account when exercising their clinical judgment, as well as in the determination and the implementation of preventive, diagnostic or therapeutic medical strategies. However, the ESC Guidelines do not override in any way whatsoever the individual responsibility of health professionals to make appropriate and accurate decisions in consideration of each patient's health condition and in consultation with that patient and the patient's caregiver where appropriate and/or necessary. It is also the health professional's responsibility to verify the rules and regulations applicable to drugs and devices at the time of prescription.

\section{Introduction}

The aim of all the ESC Guidelines is to help health professionals to make decisions in their everyday life based on the best available evidence. We will soon be celebrating the 30th anniversary of clinical trials that for the first time incontrovertibly demonstrated that the miserable outcome of patients with heart failure (HF) can be markedly improved. ${ }^{2}$ Since then, in the area of HF management we have witnessed and celebrated numerous highs, which have definitely outnumbered several lows, all of which have allowed us to unravel the pathophysiology of this clinical syndrome, but more importantly has led to better care of our patients. ${ }^{3}$ In the year 2016, no one would any longer dispute that, by applying all evidence-based discoveries, HF is now becoming a preventable and treatable disease.

The aim of this document is to provide practical, evidence-based guidelines for the diagnosis and treatment of HF. The principal changes from the 2012 guidelines relate to:

(i) a new term for patients with $\mathrm{HF}$ and a left ventricular ejection fraction (LVEF) that ranges from 40 to $49 \%$ - 'HF with midrange EF (HFmrEF)'; we believe that identifying HFmrEF as a separate group will stimulate research into the underlying characteristics, pathophysiology and treatment of this population;

(ii) clear recommendations on the diagnostic criteria for HF with reduced EF (HFrEF), HFmrEF and HF with preserved EF (HFpEF);

(iii) a new algorithm for the diagnosis of $\mathrm{HF}$ in the non-acute setting based on the evaluation of HF probability;

(iv) recommendations aimed at prevention or delay of the development of overt HF or the prevention of death before the onset of symptoms;

(v) indications for the use of the new compound sacubitril/ valsartan, the first in the class of angiotensin receptor neprilysin inhibitors (ARNIs);

(vi) modified indications for cardiac resynchronization therapy (CRT);

(vii) the concept of an early initiation of appropriate therapy going along with relevant investigations in acute HF that follows the 'time to therapy' approach already well established in acute coronary syndrome (ACS); (viii) a new algorithm for a combined diagnosis and treatment approach of acute HF based on the presence/absence of congestion/hypoperfusion.

We followed the format of the previous ESC 2012 HF Guidelines. Therapeutic recommendations state the treatment effect supported by the class and level of recommendation in tabular format; in the case of chronic HF due to left ventricular systolic dysfunction (LVSD) the recommendations focus on mortality and morbidity outcomes. Detailed summaries of the key evidence supporting generally recommended treatments have been provided. For diagnostic recommendations a level of evidence $C$ has been typically decided upon, because for the majority of diagnostic tests there are no data from randomized controlled trials (RCTs) showing that they will lead to reductions in morbidity and/or mortality. Practical guidance is provided for the use of the important disease-modifying drugs and diuretics. When possible, other relevant guidelines, consensus statements and position papers have been cited to avoid unduly lengthy text. All tables should be read in conjunction with their accompanying text and not read in isolation.

This document is the result of extensive interactions between the Task Force, the review team and the ESC Committee for Practice Guidelines. It represents a consensus of opinion of all of the experts involved in its development. Concurrently to the development of the 2016 ESC Guidelines on HF, the group writing the "2016 ACC/AHA/HFSA Focused Update on New Pharmacological Therapy for Heart Failure" independently developed its recommendations on new pharmacotherapy for Heart Failure. Both working groups/Task Force independently surveyed the evidence, arrived at similar conclusions, and constructed similar, but not identical, recommendations. Given the concordance, the respective organizations simultaneously issued aligned recommendations on the use of these new treatments to minimize confusion and improve the care of patients with HF.

\section{Definition, epidemiology and prognosis}

\subsection{Definition of heart failure}

$\mathrm{HF}$ is a clinical syndrome characterized by typical symptoms (e.g. breathlessness, ankle swelling and fatigue) that may be accompanied by signs (e.g. elevated jugular venous pressure, pulmonary crackles and peripheral oedema) caused by a structural and/or functional cardiac abnormality, resulting in a reduced cardiac output and/ or elevated intracardiac pressures at rest or during stress.

The current definition of HF restricts itself to stages at which clinical symptoms are apparent. Before clinical symptoms become apparent, patients can present with asymptomatic structural or functional cardiac abnormalities [systolic or diastolic left ventricular (LV) dysfunction], which are precursors of HF. Recognition of these precursors is important because they are related to poor outcomes, and starting treatment at the precursor stage may reduce mortality in patients with asymptomatic systolic LV dysfunction ${ }^{4,5}$ (for details see Section 6).

Demonstration of an underlying cardiac cause is central to the diagnosis of HF. This is usually a myocardial abnormality causing systolic and/or diastolic ventricular dysfunction. However, abnormalities of the valves, pericardium, endocardium, heart rhythm and 
conduction can also cause HF (and more than one abnormality is often present). Identification of the underlying cardiac problem is crucial for therapeutic reasons, as the precise pathology determines the specific treatment used (e.g. valve repair or replacement for valvular disease, specific pharmacological therapy for HF with reduced EF, reduction of heart rate in tachycardiomyopathy, etc).

\subsection{Terminology}

\subsubsection{Heart failure with preserved, mid-range and reduced ejection fraction}

The main terminology used to describe HF is historical and is based on measurement of the LVEF. HF comprises a wide range of patients, from those with normal LVEF [typically considered as $\geq 50 \%$; HF with preserved EF (HFpEF)] to those with reduced LVEF [typically considered as < $40 \%$; HF with reduced EF (HFrEF)] (Table 3.1). Patients with an LVEF in the range of 40-49\% represent a 'grey area', which we now define as HFmrEF (Table 3.1). Differentiation of patients with HF based on LVEF is important due to different underlying aetiologies, demographics, co-morbidities and response to therapies. ${ }^{6}$ Most clinical trials published after 1990 selected patients based on LVEF [usually measured using echocardiography, a radionuclide technique or cardiac magnetic resonance (CMR)], and it is only in patients with HFrEF that therapies have been shown to reduce both morbidity and mortality.

The diagnosis of HFpEF is more challenging than the diagnosis of HFrEF. Patients with HFpEF generally do not have a dilated LV, but instead often have an increase in LV wall thickness and/or increased left atrial (LA) size as a sign of increased filling pressures. Most have additional 'evidence' of impaired LV filling or suction capacity, also classified as diastolic dysfunction, which is generally accepted as the likely cause of HF in these patients (hence the term 'diastolic HF'). However, most patients with HFrEF (previously referred to as 'systolic HF') also have diastolic dysfunction, and subtle abnormalities of systolic function have been shown in patients with HFpEF. Hence the preference for stating preserved or reduced LVEF over preserved or reduced 'systolic function'.

In previous guidelines it was acknowledged that a grey area exists between HFrEF and HFpEF.' These patients have an LVEF that ranges from 40 to $49 \%$, hence the term HFmrEF. Identifying HFmrEF as a separate group will stimulate research into the underlying characteristics, pathophysiology and treatment of this group of patients. Patients with HFmrEF most probably have primarily mild systolic dysfunction, but with features of diastolic dysfunction (Table 3.1).

Patients without detectable LV myocardial disease may have other cardiovascular causes for HF (e.g. pulmonary hypertension, valvular heart disease, etc.). Patients with non-cardiovascular pathologies (e.g. anaemia, pulmonary, renal or hepatic disease) may have symptoms similar or identical to those of HF and each may complicate or exacerbate the HF syndrome.

\subsubsection{Terminology related to the time course of heart failure}

In these guidelines, the term HF is used to describe the symptomatic syndrome, graded according to the New York Heart Association (NYHA) functional classification (see Section 3.2.3 and Web Table 3.2), although a patient can be rendered asymptomatic by treatment. In these guidelines, a patient who has never exhibited the typical symptoms and/or signs of HF and with a reduced LVEF is described as having asymptomatic LV systolic dysfunction. Patients who have had HF for some time are often said to have 'chronic HF'. A treated patient with symptoms and signs that have remained generally unchanged for at least 1 month is said to be 'stable'. If chronic stable HF deteriorates, the patient may be described as 'decompensated' and this may happen suddenly or slowly, often leading to hospital admission, an event of considerable prognostic importance. New-onset ('de novo') HF may also present acutely, for example, as a consequence of acute myocardial infarction (AMI), or in a subacute (gradual) fashion, for example, in patients with a dilated cardiomyopathy (DCM), who often have symptoms for weeks or months before the diagnosis becomes clear. Although symptoms and signs of HF may resolve, the underlying cardiac dysfunction may not, and patients remain at the risk of recurrent 'decompensation'.

Occasionally, however, a patient may have HF due to a problem that resolves completely (e.g. acute viral myocarditis, takotsubo cardiomyopathy or tachycardiomyopathy). Other patients, particularly those with 'idiopathic' DCM, may also show substantial or even complete recovery of LV systolic function with modern diseasemodifying therapy [including angiotensin-converting enzyme inhibitor (ACEI), beta-blocker, mineralocorticoid receptor antagonist

\section{Table 3.I Definition of heart failure with preserved (HFpEF), mid-range (HFmrEF) and reduced ejection fraction (HFrEF)}

\begin{tabular}{|c|c|c|c|c|}
\hline \multicolumn{2}{|c|}{ Type of HF } & HFrEF & HFmrEF & HFpEF \\
\hline \multirow{3}{*}{ 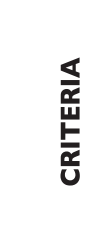 } & $\mathbf{I}$ & Symptoms \pm Signs ${ }^{\mathrm{a}}$ & Symptoms \pm Signs ${ }^{\mathrm{a}}$ & Symptoms \pm Signs ${ }^{\mathrm{a}}$ \\
\hline & 2 & LVEF $<40 \%$ & LVEF $40-49 \%$ & LVEF $\geq 50 \%$ \\
\hline & 3 & - & $\begin{array}{l}\text { I. Elevated levels of natriuretic peptides } \\
\text { 2. At least one additional criterion: } \\
\text { a. relevant structural heart disease (LVH and/or LAE), } \\
\text { b. diastolic dysfunction (for details see Section 4.3.2). }\end{array}$ & $\begin{array}{l}\text { I. Elevated levels of natriuretic peptides }{ }^{\mathrm{b}} \text {; } \\
\text { 2. At least one additional criterion: } \\
\text { a. relevant structural heart disease (LVH and/or LAE), } \\
\text { b. diastolic dysfunction (for details see Section 4.3.2). }\end{array}$ \\
\hline
\end{tabular}

$\mathrm{BNP}=\mathrm{B}$-type natriuretic peptide; $\mathrm{HF}=$ heart failure; $\mathrm{HFmrEF}=$ heart failure with mid-range ejection fraction; $\mathrm{HFpEF}=$ heart failure with preserved ejection fraction; $\mathrm{HFrEF}=$ heart failure with reduced ejection fraction; LAE = left atrial enlargement; LVEF = left ventricular ejection fraction; LVH = left ventricular hypertrophy; NT-proBNP = N-terminal pro-B type natriuretic peptide.

${ }^{\text {a }}$ igns may not be present in the early stages of HF (especially in HFpEF) and in patients treated with diuretics.

${ }^{b} \mathrm{BNP}>35 \mathrm{pg} / \mathrm{ml}$ and/or NT-proBNP> $125 \mathrm{pg} / \mathrm{mL}$. 
(MRA), ivabradine and/or CRT]. 'Congestive HF' is a term that is sometimes used, and may describe acute or chronic HF with evidence of volume overload. Many or all of these terms may be accurately applied to the same patient at different times, depending upon their stage of illness.

\subsubsection{Terminology related to the symptomatic severity of heart failure}

The NYHA functional classification (Web Table 3.2) has been used to describe the severity of symptoms and exercise intolerance. However, symptom severity correlates poorly with many measures of $L V$ function; although there is a clear relationship between the severity of symptoms and survival, patients with mild symptoms may still have an increased risk of hospitalization and death. ${ }^{8-10}$

Sometimes the term 'advanced HF' is used to characterize patients with severe symptoms, recurrent decompensation and severe cardiac dysfunction. ${ }^{11}$ The American College of Cardiology Foundation/American Heart Association (ACCF/AHA) classification describes stages of HF development based on structural changes and symptoms (Web Table 3.3). ${ }^{12}$ The Killip classification may be used to describe the severity of the patient's condition in the acute setting after myocardial infarction (see Section 12). ${ }^{13}$

\subsection{Epidemiology, aetiology and natural history of heart failure}

The prevalence of HF depends on the definition applied, but is approximately $1-2 \%$ of the adult population in developed countries, rising to $\geq 10 \%$ among people $>70$ years of age. ${ }^{14-17}$ Among people $>65$ years of age presenting to primary care with breathlessness on exertion, one in six will have unrecognized HF (mainly HFpEF). ${ }^{18,19}$ The lifetime risk of HF at age 55 years is $33 \%$ for men and $28 \%$ for women. ${ }^{16}$ The proportion of patients with HFpEF ranges from 22 to $73 \%$, depending on the definition applied, the clinical setting (primary care, hospital clinic, hospital admission), age and sex of the studied population, previous myocardial infarction and the year of publication. ${ }^{17,18,20-30}$

Data on temporal trends based on hospitalized patients suggest that the incidence of HF may be decreasing, more for HFrEF than for HFpEF. ${ }^{31,32}$ HFpEF and HFrEF seem to have different epidemiological and aetiological profiles. Compared with HFrEF, patients with HFpEF are older, more often women and more commonly have a history of hypertension and atrial fibrillation (AF), while a history of myocardial infarction is less common. ${ }^{32,33}$ The characteristics of patients with HFmrEF are between those with HFrEF and HFpEF, ${ }^{34}$ but further studies are needed to better characterize this population.

The aetiology of HF is diverse within and among world regions. There is no agreed single classification system for the causes of HF, with much overlap between potential categories (Table 3.4). Many patients will have several different pathologies_cardiovascular and non-cardiovascular - that conspire to cause HF. Identification of these diverse pathologies should be part of the diagnostic workup, as they may offer specific therapeutic opportunities.

Many patients with HF and ischaemic heart disease (IHD) have a history of myocardial infarction or revascularization. However, a normal coronary angiogram does not exclude myocardial scar (e.g. by CMR imaging) or impaired coronary microcirculation as alternative evidence for IHD.
In clinical practice, a clear distinction between acquired and inherited cardiomyopathies remains challenging. In most patients with a definite clinical diagnosis of $\mathrm{HF}$, there is no confirmatory role for routine genetic testing, but genetic counselling is recommended in patients with hypertrophic cardiomyopathy (HCM), 'idiopathic' DCM or arrhythmogenic right ventricular cardiomyopathy (ARVC) (see Section 5.10.1), since the outcomes of these tests may have clinical implications.

Over the last 30 years, improvements in treatments and their implementation have improved survival and reduced the hospitalization rate in patients with $\mathrm{HFrEF}$, although the outcome often remains unsatisfactory. The most recent European data (ESC-HF pilot study) demonstrate that 12-month all-cause mortality rates for hospitalized and stable/ambulatory HF patients were $17 \%$ and $7 \%$, respectively, and the 12 -month hospitalization rates were $44 \%$ and $32 \%$, respectively. ${ }^{35}$ In patients with HF (both hospitalized and ambulatory), most deaths are due to cardiovascular causes, mainly sudden death and worsening HF. All-cause mortality is generally higher in HFrEF than HFpEF. ${ }^{35,36}$ Hospitalizations are often due to non-cardiovascular causes, particularly in patients with HFpEF. Hospitalization for cardiovascular causes did not change from 2000 to 2010, whereas those with non-cardiovascular causes increased. ${ }^{31}$

\subsection{Prognosis}

Estimation of prognosis for morbidity, disability and death helps patients, their families and clinicians decide on the appropriate type and timing of therapies (in particular, decisions about a rapid transition to advanced therapies) and assists with planning of health and social services and resources.

Numerous prognostic markers of death and/or HF hospitalization have been identified in patients with HF (Web Table 3.5). However, their clinical applicability is limited and precise risk stratification in HF remains challenging.

In recent decades, several multivariable prognostic risk scores have been developed for different populations of patients with $\mathrm{HF}^{36-41}$ and some are available as interactive online applications. Multivariable risk scores may help predict death in patients with HF, but remain less useful for the prediction of subsequent HF hospitalizations. ${ }^{37,38}$ A systematic review examining 64 prognostic models $^{37}$ along with a meta-analysis and meta-regression study of 117 prognostic models ${ }^{38}$ revealed only a moderate accuracy of models predicting mortality, whereas models designed to predict the combined endpoint of death or hospitalization, or only hospitalization, had an even poorer discriminative ability.

\section{Diagnosis}

\subsection{Symptoms and signs}

Symptoms are often non-specific and do not, therefore, help discriminate between $\mathrm{HF}$ and other problems (Table 4.1). ${ }^{42-46}$ Symptoms and signs of HF due to fluid retention may resolve quickly with diuretic therapy. Signs, such as elevated jugular venous pressure and displacement of the apical impulse, may be more specific, but are harder to detect and have poor reproducibility. ${ }^{18,46,47}$ Symptoms and signs may be particularly difficult to identify and interpret in obese individuals, in the elderly and in patients with chronic lung disease. ${ }^{48-50}$ Younger patients with HF often have a different aetiology, clinical presentation and outcome compared with older patients. ${ }^{51,52}$ 
Table 3.4 Aetiologies of heart failure

\begin{tabular}{|c|c|c|}
\hline \multicolumn{3}{|c|}{ DISEASED MYOCARDIUM } \\
\hline \multirow{5}{*}{$\begin{array}{l}\text { Ischaemic heart } \\
\text { disease }\end{array}$} & Myocardial scar & \\
\hline & Myocardial stunning/hibernation & \\
\hline & Epicardial coronary artery disease & \\
\hline & Abnormal coronary microcirculation & \\
\hline & Endothelial dysfunction & \\
\hline \multirow[t]{4}{*}{ Toxic damage } & Recreational substance abuse & Alcohol, cocaine, amphetamine, anabolic steroids. \\
\hline & Heavy metals & Copper, iron, lead, cobalt. \\
\hline & Medications & $\begin{array}{l}\text { Cytostatic drugs (e.g. anthracyclines), immunomodulating drugs (e.g. interferons monoclonal } \\
\text { antibodies such as trastuzumab, cetuximab), antidepressant drugs, antiarrhythmics, non-steroidal } \\
\text { anti-Inflammatory drugs, anaesthetics. }\end{array}$ \\
\hline & Radiation & \\
\hline \multirow{2}{*}{$\begin{array}{l}\text { Immune-mediated } \\
\text { and inflammatory } \\
\text { damage }\end{array}$} & Related to infection & Bacteria, spirochaetes, fungi, protozoa, parasites (Chagas disease), rickettsiae, viruses (HIV/AIDS). \\
\hline & Not related to infection & $\begin{array}{l}\text { Lymphocytic/giant cell myocarditis, autoimmune diseases (e.g. Graves' disease, rheumatoid } \\
\text { arthritis, connective tissue disorders, mainly systemic lupus erythematosus), hypersensitivity and } \\
\text { eosinophilic myocarditis (Churg-Strauss). }\end{array}$ \\
\hline \multirow[t]{2}{*}{ Infiltration } & Related to malignancy & Direct infiltrations and metastases. \\
\hline & Not related to malignancy & $\begin{array}{l}\text { Amyloidosis, sarcoidosis, haemochromatosis (iron), glycogen storage diseases (e.g. Pompe disease), } \\
\text { lysosomal storage diseases (e.g. Fabry disease). }\end{array}$ \\
\hline \multirow[t]{2}{*}{$\begin{array}{l}\text { Metabolic } \\
\text { derangements }\end{array}$} & Hormonal & $\begin{array}{l}\text { Thyroid diseases, parathyroid diseases, acromegaly, GH deficiency, hypercortisolaemia, Conn's } \\
\text { disease,Addison disease, diabetes, metabolic syndrome, phaeochromocytoma, pathologies related } \\
\text { to pregnancy and peripartum. }\end{array}$ \\
\hline & Nutritional & $\begin{array}{l}\text { Deficiencies in thiamine, L-carnitine, selenium, iron, phosphates, calcium, complex malnutrition } \\
\text { (e.g. malignancy,AIDS, anorexia nervosa), obesity. }\end{array}$ \\
\hline Genetic abnormalities & Diverse forms & $\begin{array}{l}\text { HCM, DCM, LV non-compaction,ARVC, restrictive cardiomyopathy (for details see respective } \\
\text { expert documents), muscular dystrophies and laminopathies. }\end{array}$ \\
\hline \multicolumn{3}{|c|}{ ABNORMAL LOADING CONDITIONS } \\
\hline \multicolumn{3}{|l|}{ Hypertension } \\
\hline \multirow{2}{*}{$\begin{array}{l}\text { Valve and } \\
\text { myocardium } \\
\text { structural defects }\end{array}$} & Acquired & Mitral, aortic, tricuspid and pulmonary valve diseases. \\
\hline & Congenital & Atrial and ventricular septum defects and others (for details see a respective expert document). \\
\hline \multirow{2}{*}{$\begin{array}{l}\text { Pericardial and } \\
\text { endomyocardial } \\
\text { pathologies }\end{array}$} & Pericardial & $\begin{array}{l}\text { Constrictive pericarditis } \\
\text { Pericardial effusion }\end{array}$ \\
\hline & Endomyocardial & HES, EMF, endocardial fibroelastosis. \\
\hline High output states & & Severe anaemia, sepsis, thyrotoxicosis, Paget's disease, arteriovenous fistula, pregnancy. \\
\hline Volume overload & & Renal failure, iatrogenic fluid overload. \\
\hline \multicolumn{3}{|l|}{ ARRHYTHMIAS } \\
\hline Tachyarrhythmias & & Atrial, ventricular arrhythmias. \\
\hline Bradyarrhythmias & & Sinus node dysfunctions, conduction disorders. \\
\hline
\end{tabular}

ARVC = arrhythmogenic right ventricular cardiomyopathy; DCM = dilated cardiomyopathy; $\mathrm{EMF}=$ endomyocardial fibrosis; $\mathrm{GH}=$ growth hormone; $\mathrm{HCM}=$ hypertrophic cardiomyopathy; HES = hypereosinophilic syndrome; HIVIAIDS = human immunodeficiency virus/acquired immune deficiency syndrome; $L V=$ left ventricular.

A detailed history should always be obtained. HF is unusual in an individual with no relevant medical history (e.g. a potential cause of cardiac damage), whereas certain features, particularly previous myocardial infarction, greatly increase the likelihood of HF in a patient with appropriate symptoms and signs. ${ }^{42-45}$

At each visit, symptoms and signs of HF need to be assessed, with particular attention to evidence of congestion. Symptoms and signs are important in monitoring a patient's response to treatment and stability over time. Persistence of symptoms despite treatment usually indicates the need for additional therapy, and worsening of symptoms is a serious development (placing the patient at risk of urgent hospital admission and death) and merits prompt medical attention.

\subsection{Essential initial investigations: natriuretic peptides, electrocardiogram and echocardiography}

The plasma concentration of natriuretic peptides (NPs) can be used as an initial diagnostic test, especially in the non-acute setting when echocardiography is not immediately available. Elevated NPs help establish an initial working diagnosis, identifying those who require further cardiac investigation; patients with values below the cutpoint for the exclusion of important cardiac dysfunction do not require echocardiography (see also Section 4.3 and Section 12). Patients with normal plasma NP concentrations are unlikely to have HF. The upper limit of normal in the non-acute setting for 
Table 4.I Symptoms and signs typical of heart failure

\begin{tabular}{|l|l|}
\hline Symptoms & Signs \\
\hline Typical & More specific \\
\hline Breathlessness & Elevated jugular venous pressure \\
Orthopnoea & Hepatojugular reflux \\
Paroxysmal nocturnal dyspnoea & Third heart sound (gallop rhythm) \\
Reduced exercise tolerance & Laterally displaced apical impulse \\
Fatigue, tiredness, increased time & \\
to recover after exercise & \\
Ankle swelling & \\
\hline Less typical & Less specific \\
\hline Nocturnal cough & Weight gain (>2 kg/week) \\
Wheezing & Weight loss (in advanced HF) \\
Bloated feeling & Tissue wasting (cachexia) \\
Loss of appetite & Cardiac murmur \\
Confusion (especially in the & Peripheral oedema (ankle, sacral, \\
elderly) & scrotal) \\
Depression & Pulmonary crepitations \\
Palpitations & Reduced air entry and dullness to \\
Dizziness & percussion at lung bases (pleural \\
Syncope & effusion) \\
Bendopnea & Tachycardia \\
& Irregular pulse \\
& Tachypnoea \\
& Cheyne Stokes respiration \\
& Hepatomegaly \\
& Ascites \\
& Cold extremities \\
Oliguria \\
Narrow pulse pressure \\
\hline
\end{tabular}

$\mathrm{HF}=$ heart failure

B-type natriuretic peptide (BNP) is $35 \mathrm{pg} / \mathrm{mL}$ and for $\mathrm{N}$-terminal pro-BNP (NT-proBNP) it is $125 \mathrm{pg} / \mathrm{mL}$; in the acute setting, higher values should be used [BNP $<100 \mathrm{pg} / \mathrm{mL}$, NT-proBNP $<300 \mathrm{pg} /$ $\mathrm{mL}$ and mid-regional pro A-type natriuretic peptide (MR-proANP) $<120 \mathrm{pmol} / \mathrm{L}$ ]. Diagnostic values apply similarly to HFrEF and HFpEF; on average, values are lower for HFpEF than for HFrEF. ${ }^{54,55}$ At the mentioned exclusionary cut-points, the negative predictive values are very similar and high $(0.94-0.98)$ in both the non-acute and acute setting, but the positive predictive values are lower both in the non-acute setting $(0.44-0.57)$ and in the acute setting $(0.66-0.67) .{ }^{54,56-61}$ Therefore, the use of NPs is recommended for ruling-out HF, but not to establish the diagnosis.

There are numerous cardiovascular and non-cardiovascular causes of elevated NPs that may weaken their diagnostic utility in HF. Among them, AF, age and renal failure are the most important factors impeding the interpretation of NP measurements. ${ }^{55}$ On the other hand, NP levels may be disproportionally low in obese patients $^{62}$ (see also Section 12.2 and Table 12.3).

An abnormal electrocardiogram (ECG) increases the likelihood of the diagnosis of HF, but has low specificity. ${ }^{18,46,63,64}$ Some abnormalities on the ECG provide information on aetiology (e.g. myocardial infarction), and findings on the ECG might provide indications for therapy (e.g. anticoagulation for AF, pacing for bradycardia, CRT if broadened QRS complex) (see Sections 8 and 10). HF is unlikely in patients presenting with a completely normal ECG (sensitivity $89 \%) .{ }^{43}$ Therefore, the routine use of an ECG is mainly recommended to rule out HF.
Echocardiography is the most useful, widely available test in patients with suspected HF to establish the diagnosis. It provides immediate information on chamber volumes, ventricular systolic and diastolic function, wall thickness, valve function and pulmonary hypertension. ${ }^{65-74}$ This information is crucial in establishing the diagnosis and in determining appropriate treatment (see Sections 5.2-5.4 for details on echocardiography).

The information provided by careful clinical evaluation and the above mentioned tests will permit an initial working diagnosis and treatment plan in most patients. Other tests are generally required only if the diagnosis remains uncertain (e.g. if echocardiographic images are suboptimal or an unusual cause of $\mathrm{HF}$ is suspected) (for details see Sections 5.5-5.10).

\subsection{Algorithm for the diagnosis of heart failure}

\subsubsection{Algorithm for the diagnosis of heart failure in the non-acute setting}

An algorithm for the diagnosis of $\mathrm{HF}$ in the non-acute setting is shown in Figure 4.1. The diagnosis of HF in the acute setting is discussed in Section 12.

For patients presenting with symptoms or signs for the first time, non-urgently in primary care or in a hospital outpatient clinic (Table 4.1), the probability of HF should first be evaluated based on the patient's prior clinical history [e.g. coronary artery disease (CAD), arterial hypertension, diuretic use], presenting symptoms (e.g. orthopnoea), physical examination (e.g. bilateral oedema, increased jugular venous pressure, displaced apical beat) and resting ECG. If all elements are normal, $\mathrm{HF}$ is highly unlikely and other diagnoses need to be considered. If at least one element is abnormal, plasma NPs should be measured, if available, to identify those who need echocardiography (an echocardiogram is indicated if the NP level is above the exclusion threshold or if circulating NP levels cannot be assessed). ${ }^{55-60,75-78}$

\subsubsection{Diagnosis of heart failure with preserved ejection fraction}

The diagnosis of HFpEF remains challenging. LVEF is normal and signs and symptoms for HF (Table 4.1) are often non-specific and do not discriminate well between $\mathrm{HF}$ and other clinical conditions. This section summarizes practical recommendations necessary for proper diagnosis of this clinical entity in clinical practice.

The diagnosis of chronic HFpEF, especially in the typical elderly patient with co-morbidities and no obvious signs of central fluid overload, is cumbersome and a validated gold standard is missing. To improve the specificity of diagnosing HFpEF, the clinical diagnosis needs to be supported by objective measures of cardiac dysfunction at rest or during exercise. The diagnosis of HFpEF requires the following conditions to be fulfilled (see Table 3.1):

- The presence of symptoms and/or signs of HF (see Table 4.1)

- A 'preserved' EF (defined as LVEF $\geq 50 \%$ or $40-49 \%$ for HFmrEF)

- Elevated levels of NPs (BNP $>35 \mathrm{pg} / \mathrm{mL}$ and/or NT-proBNP $>125 \mathrm{pg} / \mathrm{mL}$ )

- Objective evidence of other cardiac functional and structural alterations underlying HF (for details, see below) 


\section{PATIENT WITH SUSPECTED HFa}

(non-acute onset)

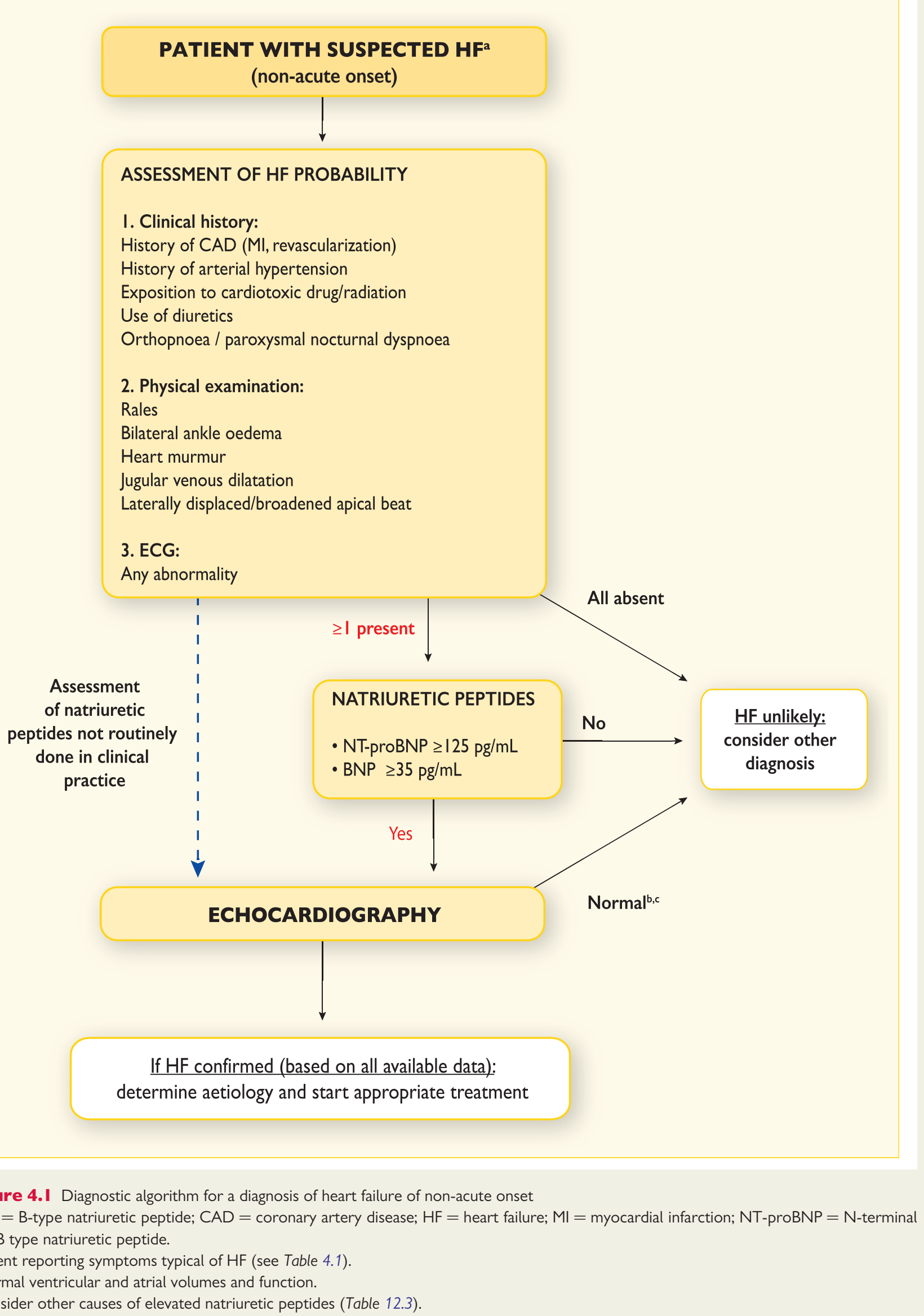


- In case of uncertainty, a stress test or invasively measured elevated LV filling pressure may be needed to confirm the diagnosis (for details, see below).

The initial assessment consists of a clinical diagnosis compatible with the algorithm presented above and the assessment of LVEF by echocardiography. The cut-off of $50 \%$ for a diagnosis of HFpEF is arbitrary; patients with an LVEF between 40 and $49 \%$ are often classified as HFpEF in clinical trials. ${ }^{79}$ However, in the present guidelines, we define HFpEF as an LVEF $\geq 50 \%$ and consider patients with an LVEF between 40 and $49 \%$ as a grey area, which could be indicated as HFmrEF. Clinical signs and symptoms are similar for patients with HFrEF, HFmrEF and HFpEF. Typical demographics and co-morbidities are provided in Web Table 4.2. The resting ECG may reveal abnormalities such as AF, LV hypertrophy and repolarisation abnormalities. A normal ECG and/or plasma concentrations of $\mathrm{BNP}<35 \mathrm{pg} / \mathrm{mL}$ and/or NT-proBNP $<125 \mathrm{pg} / \mathrm{mL}$ make a diagnosis of HFpEF, HFmrEF or HFrEF unlikely.

The next step comprises an advanced workup in case of initial evidence of HFpEF/HFmrEF and consists of objective demonstration of structural and/or functional alterations of the heart as the underlying cause for the clinical presentation. Key structural alterations are a left atrial volume index $(\mathrm{LAVI})>34 \mathrm{~mL} / \mathrm{m}^{2}$ or a left ventricular mass index $\left(\right.$ LVMI) $\geq 115 \mathrm{~g} / \mathrm{m}^{2}$ for males and $\geq 95 \mathrm{~g} / \mathrm{m}^{2}$ for females. ${ }^{65,67,72}$ Key functional alterations are an $E / e^{\prime} \geq 13$ and a mean e' septal and lateral wall $<9 \mathrm{~cm} / \mathrm{s}^{65,67,70,72,80-84}$ Other (indirect) echocardiographically derived measurements are longitudinal strain or tricuspid regurgitation velocity (TRV). ${ }^{72,82}$ An overview of normal and abnormal values for echocardiographic parameters related to diastolic function is presented in Web Table 4.3. Not all of the recommended values are identical to those published in previous guidelines, because of the inclusion of new data published in recent reports, in particular by Cabarello et al. ${ }^{70}$

A diastolic stress test can be performed with echocardiography, typically using a semi-supine bicycle ergometer exercise protocol with assessment of LV (E/e') and pulmonary artery pressures (TRV), systolic dysfunction (longitudinal strain), stroke volume and cardiac output changes with exercise. ${ }^{85,86}$ Different dynamic exercise protocols are available, with semi-supine bicycle ergometry and echocardiography at rest and submaximal exercise being used most often. ${ }^{85}$ Exercise-induced increases in E/e' beyond diagnostic cut-offs (i.e. $>13$ ), but also other indirect measures of systolic and diastolic function, such as longitudinal strain or TRV, are used. Alternatively, invasive haemodynamics at rest with assessment of filling pressures [pulmonary capillary wedge pressure (PCWP) $\geq 15 \mathrm{mmHg}$ or left ventricular end diastolic pressure (LVEDP) $\geq 16 \mathrm{mmHg}$ ] followed by exercise haemodynamics if below these thresholds, with assessment of changes in filling pressures, pulmonary artery systolic pressure, stroke volume and cardiac output, can be performed. ${ }^{87}$

The diagnosis of HFpEF in patients with AF is difficult. Since AF is associated with higher NP levels, the use of NT-proBNP or BNP for diagnosing HFpEF probably needs to be stratified by the presence of sinus rhythm (with lower cut-offs) vs. AF (higher cut-offs). LAVI is increased by $A F$, and functional parameters of diastolic dysfunction are less well established in AF, and other cut-off values probably apply. On the other hand, AF might be a sign of the presence of HFpEF, and patients with $\mathrm{AF}$ and $\mathrm{HFpEF}$ often have similar patient characteristics. In addition, patients with HFpEF and AF might have more advanced HF compared with patients with HFpEF and sinus rhythm.
Patients with HFpEF are a heterogeneous group with various underlying aetiologies and pathophysiological abnormalities. Based on specific suspected causes, additional tests can be performed (Web Table 4.4). ${ }^{71,88-94}$ However, they can only be recommended if the results might affect management.

\section{Cardiac imaging and other diagnostic tests}

Cardiac imaging plays a central role in the diagnosis of HF and in guiding treatment. Of several imaging modalities available, echocardiography is the method of choice in patients with suspected HF, for reasons of accuracy, availability (including portability), safety and cost. ${ }^{68,69,72}$ Echocardiography may be complemented by other modalities, chosen according to their ability to answer specific clinical questions and taking account of contraindications to and risks of specific tests. ${ }^{71,73}$

In general, imaging tests should only be performed when they have a meaningful clinical consequence. The reliability of the outcomes is highly dependent on the imaging modality, the operator and centre experience and imaging quality. Normal values may vary with age, sex and imaging modality.

\subsection{Chest X-ray}

A chest $X$-ray is of limited use in the diagnostic work-up of patients with suspected HF. It is probably most useful in identifying an alternative, pulmonary explanation for a patient's symptoms and signs, i.e. pulmonary malignancy and interstitial pulmonary disease, although computed tomography (CT) of the chest is currently the standard of care. For the diagnosis of asthma or chronic obstructive pulmonary disease (COPD), pulmonary function testing with spirometry is needed. The chest $\mathrm{X}$-ray may, however, show pulmonary venous congestion or oedema in a patient with $\mathrm{HF}$, and is more helpful in the acute setting than in the non-acute setting. ${ }^{49,64}$ It is important to note that significant LV dysfunction may be present without cardiomegaly on the chest X-ray. ${ }^{49,64}$

\subsection{Transthoracic echocardiography}

Echocardiography is a term used here to refer to all cardiac ultrasound imaging techniques, including two-dimensional/threedimensional echocardiography, pulsed and continuous wave Doppler, colour flow Doppler, tissue Doppler imaging (TDI) contrast echocardiography and deformation imaging (strain and strain rate).

Transthoracic echocardiography (TTE) is the method of choice for assessment of myocardial systolic and diastolic function of both left and right ventricles.

\subsubsection{Assessment of left ventricular systolic function}

For measurement of LVEF, the modified biplane Simpson's rule is recommended. LV end diastolic volume (LVEDV) and LV end systolic volume (LVESV) are obtained from apical four- and two-chamber views. This method relies on accurate tracing of endocardial borders. In case of poor image quality, contrast agents should be used to improve endocardial delineation. ${ }^{72}$ Measurement of regional wall motion abnormalities might be particularly relevant for patients suspected of CAD or myocarditis.

The Teichholz and Quinones methods of calculating LVEF from linear dimensions, as well as a measurement of fractional shortening, are not recommended, as they may result in inaccuracies, 
particularly in patients with regional LV dysfunction and/or LV remodelling. Three-dimensional echocardiography of adequate quality improves the quantification of LV volumes and LVEF and has the best accuracy compared with values obtained through CMR. ${ }^{95}$

Doppler techniques allow the calculation of haemodynamic variables, such as stroke volume index and cardiac output, based on the velocity time integral at the LV outflow tract area.

In recent years, tissue Doppler parameters ( $\mathrm{S}$ wave) and deformation imaging techniques (strain and strain rate) have been shown to be reproducible and feasible for clinical use, especially in detecting subtle abnormalities in systolic function in the preclinical stage; however, measurements may vary among vendors and software versions. ${ }^{74}$

\subsubsection{Assessment of left ventricular diastolic function}

LV diastolic dysfunction is thought to be the underlying pathophysiological abnormality in patients with HFpEF and perhaps HFmrEF, and thus its assessment plays an important role in diagnosis. Although echocardiography is at present the only imaging technique that can allow for the diagnosis of diastolic dysfunction, no single echocardiography variable is sufficiently accurate to be used in isolation to make a diagnosis of LV diastolic dysfunction. Therefore, a comprehensive echocardiography examination incorporating all relevant twodimensional and Doppler data is recommended (see Section 4.3.2).

\subsubsection{Assessment of right ventricular function and pulmonary arterial pressure}

An obligatory element of echocardiography examination is the assessment of right ventricle (RV) structure and function, including $\mathrm{RV}$ and right atrial (RA) dimensions, an estimation of RV systolic function and pulmonary arterial pressure. Among parameters reflecting RV systolic function, the following measures are of particular importance: tricuspid annular plane systolic excursion (TAPSE; abnormal TAPSE $<17 \mathrm{~mm}$ indicates RV systolic dysfunction) and tissue Doppler-derived tricuspid lateral annular systolic velocity $\left(\mathrm{s}^{\prime}\right)$ ( $\mathrm{s}^{\prime}$ velocity $<9.5 \mathrm{~cm} / \mathrm{s}$ indicates RV systolic dysfunction). ${ }^{72,96}$ Systolic pulmonary artery pressure is derived from an optimal recording of maximal tricuspid regurgitant jet and the tricuspid systolic gradient, together with an estimate of RA pressure on the basis of inferior vena cava (IVC) size and its breathing-related collapse. ${ }^{97} \mathrm{RV}$ size should be routinely assessed by conventional twodimensional echocardiography using multiple acoustic windows, and the report should include both qualitative and quantitative parameters. In laboratories with experience in three-dimensional echocardiography, when knowledge of RV volumes may be clinically important, three-dimensional measurement of RV volumes is recommended. ${ }^{95}$ Three-dimensional speckle tracking echocardiography may be an additional quantitative method to assess RV function in specialised centres. ${ }^{98}$

\subsection{Transoesophageal echocardiography}

Transoesophageal echocardiography (TOE) is not needed in the routine diagnostic assessment of HF; however, it may be valuable in some clinical scenarios of patients with valve disease, suspected aortic dissection, suspected endocarditis or congenital heart disease and for ruling out intracavitary thrombi in AF patients requiring cardioversion. When the severity of mitral or aortic valve disease does not match the patient's symptoms using TTE alone, a TOE examination should be performed.

\subsection{Stress echocardiography}

Exercise or pharmacological stress echocardiography may be used for the assessment of inducible ischaemia and/or myocardium viability ${ }^{99}$ and in some clinical scenarios of patients with valve disease (e.g. dynamic mitral regurgitation, low-flow-low-gradient aortic stenosis). ${ }^{99,100}$ There are also suggestions that stress echocardiography may allow the detection of diastolic dysfunction related to exercise exposure in patients with exertional dyspnoea, preserved LVEF and inconclusive diastolic parameters at rest. ${ }^{85,86}$

\subsection{Cardiac magnetic resonance}

CMR is acknowledged as the gold standard for the measurements of volumes, mass and EF of both the left and right ventricles. It is the best alternative cardiac imaging modality for patients with nondiagnostic echocardiographic studies (particularly for imaging of the right heart) and is the method of choice in patients with complex congenital heart diseases. ${ }^{91,101,102}$

CMR is the preferred imaging method to assess myocardial fibrosis using late gadolinium enhancement (LGE) along with T1 mapping and can be useful for establishing HF aetiology. ${ }^{91,103}$ For example, CMR with LGE allows differentiation between ischaemic and non-ischaemic origins of HF and myocardial fibrosis/scars can be visualized. In addition, CMR allows the characterization of myocardial tissue of myocarditis, amyloidosis, sarcoidosis, Chagas disease, Fabry disease non-compaction cardiomyopathy and haemochromatosis. ${ }^{91,101,103,104}$

CMR may also be used for the assessment of myocardial ischaemia and viability in patients with HF and CAD (considered suitable for coronary revascularization). However, limited evidence from RCTs has failed to show that viability assessed by CMR or other means identified patients who obtained clinical benefit from revascularization. $^{105-107}$

Clinical limitations of CMR include local expertise, lower availability and higher costs compared with echocardiography, uncertainty about safety in patients with metallic implants (including cardiac devices) and less reliable measurements in patients with tachyarrhythmias. Claustrophobia is an important limitation for CMR. Linear gadoliniumbased contrast agents are contraindicated in individuals with a glomerular filtration rate (GFR) $<30 \mathrm{~mL} / \mathrm{min} / 1.73 \mathrm{~m}^{2}$, because they may trigger nephrogenic systemic fibrosis (this may be less of a concern with newer cyclic gadolinium-based contrast agents). ${ }^{108}$

\subsection{Single-photon emission computed tomography and radionuclide ventriculography}

Single-photon emission CT (SPECT) may be useful in assessing ischaemia and myocardial viability. ${ }^{109}$ Gated SPECT can also yield information on ventricular volumes and function, but exposes the patient to ionizing radiation. 3,3-diphosphono-1,2-propanodicarboxylic acid (DPD) scintigraphy may be useful for the detection of transthyretin cardiac amyloidosis. ${ }^{110}$

\subsection{Positron emission tomography}

Positron emission tomography (PET) (alone or with $\mathrm{CT}$ ) may be used to assess ischaemia and viability, but the flow tracers (N-13 ammonia or O-15 water) require an on-site cyclotron. ${ }^{92,111}$ Rubidium is an alternative tracer for ischaemia testing with PET, which can be produced locally at relatively low cost. Limited availability, radiation exposure and cost are the main limitations. 


\subsection{Coronary angiography}

Indications for coronary angiography in patients with HF are in concordance with the recommendations of other relevant ESC guidelines. ${ }^{112-114}$ Coronary angiography is recommended in patients with HF who suffer from angina pectoris recalcitrant to medical therapy, ${ }^{115}$ provided the patient is otherwise suitable for coronary revascularization. Coronary angiography is also recommended in patients with a history of symptomatic ventricular arrhythmia or aborted cardiac arrest. Coronary angiography should be considered in patients with $\mathrm{HF}$ and intermediate to high pre-test probability of $\mathrm{CAD}$ and the presence of ischaemia in non-invasive stress tests in order to establish the ischaemic aetiology and CAD severity.

\subsection{Cardiac computed tomography}

The main use of cardiac CT in patients with $\mathrm{HF}$ is as a non-invasive means to visualize the coronary anatomy in patients with HF with low intermediate pre-test probability of CAD or those with equivocal non-invasive stress tests in order to exclude the diagnosis of CAD, in the absence of relative contraindications. However, the test is only required when its results might affect a therapeutic decision.

The most important clinical indications for the applicability of certain imaging methods in patients with suspected or confirmed HF are shown in the recommendations table.

\section{Recommendations for cardiac imaging in patients with suspected or established heart failure}

\begin{tabular}{|c|c|c|c|}
\hline Recommendations & Class $^{\mathrm{a}}$ & Level $^{\mathrm{b}}$ & $\operatorname{Ref}^{c}$ \\
\hline $\begin{array}{l}\text { TTE is recommended for the assessment of myocardial structure and function in subjects with suspected HF in order to establish } \\
\text { a diagnosis of either HFrEF, HFmrEF or HFpEF. }\end{array}$ & I & C & \\
\hline $\begin{array}{l}\text { TTE is recommended to assess LVEF in order to identify patients with HF who would be suitable for evidence-based } \\
\text { pharmacological and device (ICD, CRT) treatment recommended for HFrEF. }\end{array}$ & I & C & \\
\hline $\begin{array}{l}\text { TTE is recommended for the assessment of valve disease, right ventricular function and pulmonary arterial pressure in patients with } \\
\text { an already established diagnosis of either HFrEF, HFmrEF or HFpEF in order to identify those suitable for correction of valve disease. }\end{array}$ & $\mathbf{I}$ & C & \\
\hline $\begin{array}{l}\text { TTE is recommended for the assessment of myocardial structure and function in subjects to be exposed to treatment which } \\
\text { potentially can damage myocardium (e.g. chemotherapy). }\end{array}$ & I & C & \\
\hline $\begin{array}{l}\text { Other techniques (including systolic tissue Doppler velocities and deformation indices, i.e. strain and strain rate), should be } \\
\text { considered in a TTE protocol in subjects at risk of developing HF in order to identify myocardial dysfunction at the preclinical stage. }\end{array}$ & Ila & C & \\
\hline $\begin{array}{l}\text { CMR with LGE should be considered in patients with dilated cardiomyopathy in order to distinguish between ischaemic and non- } \\
\text { ischaemic myocardial damage in case of equivocal clinical and other imaging data (taking account of cautions/contra-indications to CMR). }\end{array}$ & Ila & C & \\
\hline $\begin{array}{l}\text { CMR is recommended for the characterization of myocardial tissue in case of suspected myocarditis, amyloidosis, sarcoidosis, } \\
\text { Chagas disease, Fabry disease non-compaction cardiomyopathy, and haemochromatosis (taking account of cautions/contra- } \\
\text { indications to CMR). }\end{array}$ & $\mathbf{I}$ & C & \\
\hline $\begin{array}{l}\text { Non-invasive stress imaging (CMR, stress echocardiography, SPECT, PET) may be considered for the assessment of myocardial } \\
\text { ischaemia and viability in patients with HF and CAD (considered suitable for coronary revascularization) before the decision on } \\
\text { revascularization. }\end{array}$ & IIb & $\mathbf{B}$ & $116-118$ \\
\hline $\begin{array}{l}\text { Invasive coronary angiography is recommended in patients with HF and angina pectoris recalcitrant to pharmacological } \\
\text { therapy or symptomatic ventricular arrhythmias or aborted cardiac arrest (who are considered suitable for potential coronary } \\
\text { revascularization) in order to establish the diagnosis of CAD and its severity. }\end{array}$ & I & C & \\
\hline $\begin{array}{l}\text { Cardiac CT may be considered in patients with HF and low to intermediate pre-test probability of CAD or those with equivocal } \\
\text { non-invasive stress tests in order to rule out coronary artery stenosis. }\end{array}$ & Illb & C & \\
\hline $\begin{array}{l}\text { Reassessment of myocardial structure and function is recommended using non-invasive imaging: } \\
\text { - in patients presenting with worsening HF symptoms (including episodes of AHF) or experiencing any other } \\
\text { important cardiovascular event; } \\
\text { - in patients with HF who have received evidence-based pharmacotherapy in maximal tolerated doses, before the decision on } \\
\text { device implantation (ICD, CRT); } \\
\text { - in patients exposed to therapies which may damage the myocardium (e.g. chemotherapy) (serial assessments). }\end{array}$ & I & C & \\
\hline
\end{tabular}

$\mathrm{AHF}=$ acute heart failure; $\mathrm{CAD}=$ coronary artery disease; $\mathrm{CMR}=$ cardiac magnetic resonance; $\mathrm{CRT}=$ cardiac resynchronization therapy; $\mathrm{CT}=$ computed tomography; $\mathrm{HF}=$ heart failure; $\mathrm{HFpEF}=$ heart failure with preserved ejection fraction; $\mathrm{HFmrEF}=$ heart failure with mid-range ejection fraction; $\mathrm{HFrEF}=$ heart failure with reduced ejection fraction; $I C D=$ implantable cardioverter-defibrillator; LGE = late gadolinium enhancement; LVEF = left ventricular ejection fraction; PET = positron emission tomography; SPECT = single-photon emission computed tomography; TTE = transthoracic echocardiography.

${ }^{\mathrm{a}}$ Class of recommendation.

bLevel of evidence.

'Reference(s) supporting recommendations. 


\subsection{Other diagnostic tests}

Comprehensive assessment of patients with HF comprises, besides medical history and physical examination, including adequate imaging techniques, a set of additional diagnostic tests, i.e. laboratory variables, ECG, chest X-ray, exercise testing, invasive haemodynamic as- sessments and endomyocardial biopsy. The major typical indications are summarized in the recommendations table for diagnostic tests in patients with HF. Although there is extensive research on biomarkers in HF (e.g. ST2, galectin 3, copeptin, adrenomedullin), there is no definite evidence to recommend them for clinical practice.

\section{Recommendations for diagnostic tests in patients with heart failure}

\begin{tabular}{|c|c|c|c|}
\hline Recommendations & Class $^{a}$ & Level $^{\text {b }}$ & $\operatorname{Ref}^{c}$ \\
\hline \multicolumn{4}{|l|}{$\begin{array}{l}\text { The following diagnostic tests are recommended/should be considered for initial assessment of a patient with newly diagnosed } \\
\mathrm{HF} \text { in order to evaluate the patient's suitability for particular therapies, to detect reversible/treatable causes of HF and co- } \\
\text { morbidities interfering with HF: }\end{array}$} \\
\hline $\begin{array}{l}\text { - haemoglobin and WBC } \\
\text { - sodium, potassium, urea, creatinine (with estimated GFR) } \\
\text { - liver function tests (bilirubin,AST,ALT, GGTP) } \\
\text { - glucose, HbAIc } \\
\text { - lipid profile } \\
\text { - TSH } \\
\text { - ferritin, TSAT = TIBC }\end{array}$ & $\mathbf{I}$ & C & \\
\hline - natriuretic peptides & Ila & C & \\
\hline $\begin{array}{l}\text { Additional diagnostic tests aiming to identify other HF aetiologies and comorbidities should be considered in individual } \\
\text { patients with HF when there is a clinical suspicion of a particular pathology (see Table } 3.4 \text { on HF aetiologies). }\end{array}$ & Ila & C & \\
\hline $\begin{array}{l}\text { A I2-lead ECG is recommended in all patients with HF in order to determine heart rhythm, heart rate, QRS morphology, and } \\
\text { QRS duration, and to detect other relevant abnormalities. This information is needed to plan and monitor treatment. }\end{array}$ & I & C & \\
\hline \multicolumn{4}{|l|}{ Exercise testing in patients with HF: } \\
\hline $\begin{array}{l}\text { - is recommended as a part of the evaluation for heart transplantation and/or mechanical circulatory support } \\
\text { (cardiopulmonary exercise testing); }\end{array}$ & $\mathbf{I}$ & C & 119,120 \\
\hline - should be considered to optimize prescription of exercise training (preferably cardiopulmonary exercise testing); & Ila & C & \\
\hline - should be considered to identify the cause of unexplained dyspnoea (cardiopulmonary exercise testing). & Ila & C & \\
\hline - may be considered to detect reversible myocardial ischaemia. & Illb & C & \\
\hline $\begin{array}{l}\text { Chest radiography (X-ray) is recommended in patients with HF to detect/exclude alternative pulmonary or other diseases, } \\
\text { which may contribute to dyspnoea. It may also identify pulmonary congestion/oedema and is more useful in patients with } \\
\text { suspected HF in the acute setting. }\end{array}$ & I & C & \\
\hline \multicolumn{4}{|l|}{ Right heart catheterization with a pulmonary artery catheter: } \\
\hline - is recommended in patients with severe HF being evaluated for heart transplantation or mechanical circulatory support; & I & C & \\
\hline $\begin{array}{l}\text { - should be considered in patients with probable pulmonary hypertension assessed by echocardiography in order to confirm } \\
\text { pulmonary hypertension and its reversibility before the correction of valve/structural heart disease; }\end{array}$ & Ila & C & \\
\hline $\begin{array}{l}\text { - may be considered in order to adjust therapy in patients with HF who remain severely symptomatic despite initial } \\
\text { standard therapies and whose haemodynamic status is unclear. }\end{array}$ & Illb & C & \\
\hline $\begin{array}{l}\text { EMB should be considered in patients with rapidly progressive HF despite standard therapy when there is a probability of a } \\
\text { specific diagnosis which can be confirmed only in myocardial samples and specific therapy is available and effective. }\end{array}$ & Ila & C & 93 \\
\hline Thoracic ultrasound may be considered for the confirmation of pulmonary congestion and pleural effusion in patients with AHF. & Illb & C & $|2|$ \\
\hline Ultrasound measurement of inferior vena cava diameter may be considered for the assessment of volaemia status in patients with HF. & IIb & C & \\
\hline
\end{tabular}

$\mathrm{AHF}=$ acute heart failure; $\mathrm{ALT}=$ alanine aminotransferase; $\mathrm{AST}=$ aspartate aminotransferase; $\mathrm{BNP}=\mathrm{B}$-type natriuretic peptide; $\mathrm{ECG}=$ electrocardiogram; eGFR = estimated glomerular filtration rate; $\mathrm{EMB}=$ endomyocardial biopsy; GFR = glomerular filtration rate; GGTP = gamma-glutamyl transpeptidase; HbA1c = glycated haemoglobin; HF = heart failure; $\mathrm{HFrEF}=$ heart failure with reduced ejection fraction; $\mathrm{QRS}=\mathrm{Q}, \mathrm{R}$, and $\mathrm{S}$ waves (combination of three of the graphical deflections); TIBC $=$ total iron-binding capacity; TSAT $=$ transferrin saturation; $\mathrm{TSH}=$ thyroid-stimulating hormone; $\mathrm{WBC}=$ white blood cell.

${ }^{a}$ Class of recommendation.

bLevel of evidence.

'Reference(s) supporting recommendations.

\subsubsection{Genetic testing in heart failure}

Molecular genetic analysis in patients with cardiomyopathies is recommended when the prevalence of detectable mutations is sufficiently high and consistent to justify routine targeted genetic screening. Recommendations for genetic testing in patients with $\mathrm{HF}$ are based on the position statement of the European Society 
of Cardiology Working Group on Myocardial and Pericardial Diseases. ${ }^{94}$ In most patients with a definite clinical diagnosis of HF, there is no confirmatory role for routine genetic testing to establish the diagnosis. Genetic counselling is recommended in patients with HCM, idiopathic DCM and ARVC. Restrictive cardiomyopathy and isolated non-compaction cardiomyopathies are of a possible genetic origin and should also be considered for genetic testing.

HCM is mostly inherited as an autosomal dominant disease with variable expressivity and age-related penetrance. Currently, more than 20 genes and 1400 mutations have been identified, most of which are located in the sarcomere genes encoding cardiac $\beta$-myosin heavy chain (MYH7) and cardiac myosin binding protein C (MYBPC3). ${ }^{88,122}$

DCM is idiopathic in $50 \%$ of cases, about one-third of which are hereditary. There are already more than 50 genes identified that are associated with DCM. Many genes are related to the cytoskeleton. The most frequent ones are titin (TTN), lamin (LMNA) and desmin (DES). ${ }^{88,123}$

$A R V C$ is hereditary in most cases and is caused by gene mutations that encode elements of the desmosome. Desmosomal gene mutations explain $50 \%$ of cases and 10 genes are currently associated with the disease. ${ }^{124}$

Counselling should be performed by someone with sufficient knowledge of the specific psychological, social and medical implications of a diagnosis. Determination of the genotype is important, since some forms [e.g. mutations in LMNA and phospholamban $(P L N)]$ are related to a poorer prognosis. DNA analysis could also be of help to establish the diagnosis of rare forms, such as mitochondrial cardiomyopathies. Screening of first-degree relatives for early detection is recommended from early adolescence onwards, although earlier screening may be considered depending on the age of disease onset in other family members.

Recently, the MOGE(S) classification of inherited cardiomyopathies has been proposed, which includes the morphofunctional phenotype $(M)$, organ(s) involvement $(O)$, genetic inheritance pattern $(G)$, aetiological annotation (E), including genetic defect or underlying disease/ substrate, and the functional status $(\mathrm{S})$ of the disease. ${ }^{125}$

\section{Delaying or preventing the development of overt heart failure or preventing death before the onset of symptoms}

There is considerable evidence that the onset of HF may be delayed or prevented through interventions aimed at modifying risk factors for HF or treating asymptomatic LV systolic dysfunction (see recommendations table). Many trials show that control of hypertension will delay the onset of HF and some also show that it will prolong life. ${ }^{126-129}$ Different antihypertensive drugs [diuretics, ACEls, angiotensin receptor blockers (ARBs), beta-blockers] have been shown to be effective, especially in older people, both in patients with and without a history of myocardial infarction. ${ }^{126-128}$ Along with the ongoing discussion on optimal target blood pressure values in hypertensive non-diabetic subjects, the recent SPRINT study has already demonstrated that treating hypertension to a lower goal [systolic blood pressure (SBP) $<120 \mathrm{mmHg}$ vs. $<140 \mathrm{mmHg}$ ] in older hypertensive subjects ( $\geq 75$ years of age) or high-risk hypertensive patients reduces the risk of cardiovascular disease, death and hospitalization for HF. ${ }^{129}$

Recently, empaglifozin (an inhibitor of sodium-glucose cotransporter 2), has been shown to improve outcomes (including the reduction of mortality and HF hospitalizations) in patients with type 2 diabetes. ${ }^{130}$ Other hypoglycaemic agents have not been shown convincingly to reduce the risk of cardiovascular events and may increase the risk of HF. Intensification of hypoglycaemic therapy to drive down glycated haemoglobin $(\mathrm{HbA} 1 \mathrm{c})$ with agents other than empagliflozin does not reduce the risk of developing HF (for details see Section 11.6 on diabetes).

Although smoking cessation has not been shown to reduce the risk of developing HF, the epidemiological associations with the development of cardiovascular disease ${ }^{131}$ suggest that such advice, if followed, would be beneficial.

The association between alcohol intake and the risk of developing de novo HF is $U$-shaped, with the lowest risk with modest alcohol consumption (up to 7 drinks/week). ${ }^{132-134}$ Greater alcohol intake may trigger the development of toxic cardiomyopathy, and when present, complete abstention from alcohol is recommended.

An inverse relationship between physical activity and the risk of HF has been reported. A recent meta-analysis found that doses of physical activity in excess of the guideline recommended minimal levels may be required for more substantial reductions in HF risk. ${ }^{135}$

It has been shown that among subjects $\geq 40$ years of age with either cardiovascular risk factors or cardiovascular disease (but neither asymptomatic LV dysfunction nor overt HF), BNP-driven collaborative care between the primary care physician and the specialist cardiovascular centre may reduce the combined rates of LV systolic dysfunction and overt HF. ${ }^{136}$

Statins reduce the rate of cardiovascular events and mortality; there is also reasonable evidence that they prevent or delay the onset of HF. ${ }^{137-140}$ Neither aspirin nor other antiplatelet agents, nor revascularization, have been shown to reduce the risk of developing $\mathrm{HF}$ or mortality in patients with stable CAD. Obesity is also a risk factor for $\mathrm{HF},{ }^{141}$ but the impact of treatments of obesity on the development of $\mathrm{HF}$ is unknown.

In patients with CAD, without LV systolic dysfunction or HF, ACEls prevent or delay the onset of HF and reduce cardiovascular and allcause mortality, although the benefit may be small in the contemporary setting, especially in patients receiving aspirin. ${ }^{142}$ Up-titration of renin-angiotensin system antagonists and beta-blockers to maximum tolerated dosages may improve outcomes, including HF, in patients with increased plasma concentrations of NPs. ${ }^{136,143}$

A primary percutaneous coronary intervention $(\mathrm{PCl})$ at the earliest phase of an ST segment elevation myocardial infarction (STEMI) to reduce infarct size decreases the risk of developing a substantial reduction in LVEF and subsequent development of HFrEF. ${ }^{112}$ Initiation of an ACEl, a beta-blocker and an MRA immediately after a myocardial infarction, especially when it is associated with LV systolic dysfunction, reduces the rate of hospitalization for $\mathrm{HF}$ and mortality, ${ }^{144-148}$ as do statins. ${ }^{137-139}$

In asymptomatic patients with chronically reduced LVEF, regardless of its aetiology, an ACEI can reduce the risk of HF requiring hospitalization. ${ }^{5,144,145}$ This has not yet been shown for beta-blockers or MRAs. 
In patients with asymptomatic LV systolic dysfunction (LVEF $<30 \%$ ) of ischaemic origin who are $\geq 40$ days after an AMl, an im- plantable cardioverter-defibrillator (ICD) is recommended to prolong life. $^{149}$

\section{Recommendations to prevent or delay the development of overt heart failure or prevent death before the onset of symptoms}

\begin{tabular}{|c|c|c|c|}
\hline Recommendations & Class $^{a}$ & Level $^{\mathrm{b}}$ & $\operatorname{Ref}^{c}$ \\
\hline Treatment of hypertension is recommended to prevent or delay the onset of HF and prolong life. & $\mathbf{I}$ & A & $\begin{array}{l}126,129, \\
150,15 \mid\end{array}$ \\
\hline $\begin{array}{l}\text { Treatment with statins is recommended in patients with or at high-risk of CAD whether or not they have LV systolic } \\
\text { dysfunction, in order to prevent or delay the onset of HF and prolong life. }\end{array}$ & I & $\mathbf{A}$ & $\begin{array}{l}137-140, \\
152\end{array}$ \\
\hline $\begin{array}{l}\text { Counselling and treatment for smoking cessation and alcohol intake reduction is recommended for people who smoke or who } \\
\text { consume excess alcohol in order to prevent or delay the onset of HF. }\end{array}$ & I & c & $|3|-\mid 34$ \\
\hline Treating other risk factors of HF (e.g. obesity, dysglycaemia) should be considered in order to prevent or delay the onset of HF. & Ila & c & $\begin{array}{l}130,14 \mid, \\
153-155\end{array}$ \\
\hline Empagliflozin should be considered in patients with type 2 diabetes in order to prevent or delay the onset of HF and prolong life. & Ila & B & 130 \\
\hline $\begin{array}{l}\text { ACE-I is recommended in patients with asymptomatic LV systolic dysfunction and a history of myocardial infarction in order to } \\
\text { prevent or delay the onset of HF and prolong life. }\end{array}$ & I & $\mathbf{A}$ & $\begin{array}{l}5,144, \\
145\end{array}$ \\
\hline $\begin{array}{l}\text { ACE-I is recommended in patients with asymptomatic LV systolic dysfunction without a history of myocardial infarction, in order } \\
\text { to prevent or delay the onset of HF. }\end{array}$ & I & B & 5 \\
\hline $\begin{array}{l}\text { ACE-I should be considered in patients with stable CAD even if they do not have LV systolic dysfunction, in order to prevent } \\
\text { or delay the onset of HF. }\end{array}$ & Ila & $\mathbf{A}$ & 142 \\
\hline $\begin{array}{l}\text { Beta-blocker is recommended in patients with asymptomatic LV systolic dysfunction and a history of myocardial infarction, in } \\
\text { order to prevent or delay the onset of HF or prolong life. }\end{array}$ & I & B & 146 \\
\hline $\begin{array}{l}\text { ICD is recommended in patients: } \\
\text { a) with asymptomatic LV systolic dysfunction (LVEF } \leq 30 \% \text { ) of ischaemic origin, who are at least } 40 \text { days after acute } \\
\text { myocardial infarction, } \\
\text { b) with asymptomatic non-ischaemic dilated cardiomyopathy (LVEF } \leq 30 \% \text { ), who receive OMT therapy, } \\
\text { in order to prevent sudden death and prolong life. }\end{array}$ & I & B & $\begin{array}{c}149, \\
156-158\end{array}$ \\
\hline
\end{tabular}

$\mathrm{ACEI}=$ angiotensin-converting enzyme inhibitor; $\mathrm{CAD}=$ coronary artery disease; $\mathrm{HF}=$ heart failure; $\mathrm{ICD}=$ implantable cardioverter-defibrillator; $\mathrm{LV}=$ left ventricular; $\mathrm{LVEF}=$ left ventricular ejection fraction; OMT $=$ optimal medical therapy

${ }^{\mathrm{a} C}$ Class of recommendation.

bLevel of evidence.

${ }^{\mathrm{C}}$ Reference(s) supporting recommendations.

\section{Pharmacological treatment of heart failure with reduced ejection fraction}

\subsection{Objectives in the management of heart failure}

The goals of treatment in patients with HF are to improve their clinical status, functional capacity and quality of life, prevent hospital admission and reduce mortality. The fact that several drugs for HF have shown detrimental effects on long-term outcomes, despite showing beneficial effects on shorter-term surrogate markers, has led regulatory bodies and clinical practice guidelines to seek mortality/morbidity data for approving/recommending therapeutic interventions for HF. However, it is now recognized that preventing HF hospitalization and improving functional capacity are important benefits to be considered if a mortality excess is ruled out. ${ }^{159-161}$
Figure 7.1 shows a treatment strategy for the use of drugs (and devices) in patients with HFrEF. The recommendations for each treatment are summarized below.

Neuro-hormonal antagonists (ACEls, MRAs and beta-blockers) have been shown to improve survival in patients with HFrEF and are recommended for the treatment of every patient with HFrEF, unless contraindicated or not tolerated. A new compound (LCZ696) that combines the moieties of an ARB (valsartan) and a neprilysin (NEP) inhibitor (sacubitril) has recently been shown to be superior to an ACEI (enalapril) in reducing the risk of death and of hospitalization for HF in a single trial with strict inclusion/exclusion criteria. ${ }^{162}$ Sacubitril/valsartan is therefore recommended to replace ACEls in ambulatory HFrEF patients who remain symptomatic despite optimal therapy and who fit these trial criteria. ARBs have not been consistently proven to reduce mortality in patients with $\mathrm{HFrEF}$ and their use should be restricted to patients intolerant of an ACEl or those who take an ACEl but are unable to tolerate an 
MRA. Ivabradine reduces the elevated heart rate often seen in HFrEF and has also been shown to improve outcomes, and should be considered when appropriate.

The above medications should be used in conjunction with diuretics in patients with symptoms and/or signs of congestion. The use of diuretics should be modulated according to the patient's clinical status.

The key evidence supporting the recommendations in this section is given in Web Table 7.1. The recommended doses of these disease-modifying medications are given in Table 7.2. The recommendations given in Sections 7.5 and 7.6 summarize drugs that should be avoided or used with caution in patients with HFrEF.

\subsection{Treatments recommended in all symptomatic patients with heart failure with reduced ejection fraction}

\subsubsection{Angiotensin-converting enzyme inhibitors}

ACEls have been shown to reduce mortality and morbidity in patients with $\mathrm{HFrEF}^{2,5,163-165}$ and are recommended unless contraindicated or not tolerated in all symptomatic patients. ACEls should be up-titrated to the maximum tolerated dose in order to achieve adequate inhibition of the renin-angiotensin-aldosterone system (RAAS). There is evidence that in clinical practice the majority of patients receive suboptimal doses of ACEI. ${ }^{166}$ ACEls are also recommended in patients with asymptomatic LV systolic dysfunction to reduce the risk of HF development, HF hospitalization and death (see Section 6).

Pharmacological treatments indicated in patients with symptomatic (NYHA Class II-IV) heart failure with reduced ejection fraction

\begin{tabular}{|c|c|c|c|}
\hline Recommendations & Class $^{a}$ & Level $^{\text {b }}$ & $\operatorname{Ref}^{c}$ \\
\hline $\begin{array}{l}\text { An ACE- } I^{d} \text { is recommended, } \\
\text { in addition to a beta-blocker, } \\
\text { for symptomatic patients with } \\
\text { HFrEF to reduce the risk of HF } \\
\text { hospitalization and death. }\end{array}$ & I & $\mathbf{A}$ & $\begin{array}{c}2 \\
163 \\
-165\end{array}$ \\
\hline $\begin{array}{l}\text { A beta-blocker is recommended, } \\
\text { in addition an ACE- } I^{d} \text {, for } \\
\text { patients with stable, symptomatic } \\
\text { HFrEF to reduce the risk of HF } \\
\text { hospitalization and death. }\end{array}$ & I & $\mathbf{A}$ & $\begin{array}{c}167- \\
173\end{array}$ \\
\hline $\begin{array}{l}\text { An MRA is recommended for } \\
\text { patients with HFrEF, who remain } \\
\text { symptomatic despite treatment } \\
\text { with an ACE-I }{ }^{d} \text { and a } \\
\text { beta-blocker, to reduce the risk of } \\
\text { HF hospitalization and death. }\end{array}$ & I & $\mathbf{A}$ & 174,175 \\
\hline
\end{tabular}

$\mathrm{ACEI}=$ angiotensin-converting enzyme inhibitor; $\mathrm{HF}=$ heart failure; $\mathrm{HFrEF}=$ heart failure with reduced ejection fraction; $M R A=$ mineralocorticoid receptor antagonist; NYHA = New York Heart Association.

${ }^{\mathrm{a}} \mathrm{Class}$ of recommendation.

bLevel of evidence.

${ }^{\mathrm{C}}$ Reference(s) supporting recommendations.

${ }^{\mathrm{d}} \mathrm{Or}$ ARB if ACEl is not tolerated/contraindicated
Practical guidance on how to use ACE inhibitors is given in Web Table 7.4.

\subsubsection{Beta-blockers}

Beta-blockers reduce mortality and morbidity in symptomatic patients with HFrEF, despite treatment with an ACEI and, in most cases, a diuretic, ${ }^{167,168,170,172,173}$ but have not been tested in congested or decompensated patients. There is consensus that beta-blockers and ACEls are complementary, and can be started together as soon as the diagnosis of HFrEF is made. There is no evidence favouring the initiation of treatment with a beta-blocker before an ACEI has been started. ${ }^{176}$ Betablockers should be initiated in clinically stable patients at a low dose and gradually up-titrated to the maximum tolerated dose. In patients admitted due to acute HF (AHF) beta-blockers should be cautiously initiated in hospital, once the patient is stabilized.

An individual patient data meta-analysis of all the major betablocker trials in HFrEF has shown no benefit on hospital admissions and mortality in the subgroup of patients with HFrEF who are in $A F{ }^{177}$ However, since this is a retrospective subgroup analysis, and because beta-blockers did not increase the risk, the guideline committee decided not to make a separate recommendation according to heart rhythm. Beta-blockers should be considered for rate control in patients with HFrEF and AF, especially in those with high heart rate (see Section 10.1 for details).

Beta-blockers are recommended in patients with a history of myocardial infarction and asymptomatic LV systolic dysfunction to reduce the risk of death (see Section 6).

Practical guidance on how to use beta-blockers is given in Web Table 7.5.

\subsubsection{Mineralocorticoid/aldosterone receptor antagonists}

MRAs (spironolactone and eplerenone) block receptors that bind aldosterone and, with different degrees of affinity, other steroid hormone (e.g. corticosteroids, androgens) receptors. Spironolactone or eplerenone are recommended in all symptomatic patients (despite treatment with an ACEI and a beta-blocker) with HFrEF and LVEF $\leq 35 \%$, to reduce mortality and HF hospitalization. ${ }^{174,175}$

Caution should be exercised when MRAs are used in patients with impaired renal function and in those with serum potassium levels $>5.0 \mathrm{mmol} / \mathrm{L}$. Regular checks of serum potassium levels and renal function should be performed according to clinical status.

Practical guidance on how to use MRAs is given in Web Table 7.6.

\subsection{Other treatments recommended in selected symptomatic patients with heart failure with reduced ejection fraction \\ 7.3.1 Diuretics}

Diuretics are recommended to reduce the signs and symptoms of congestion in patients with HFrEF, but their effects on 

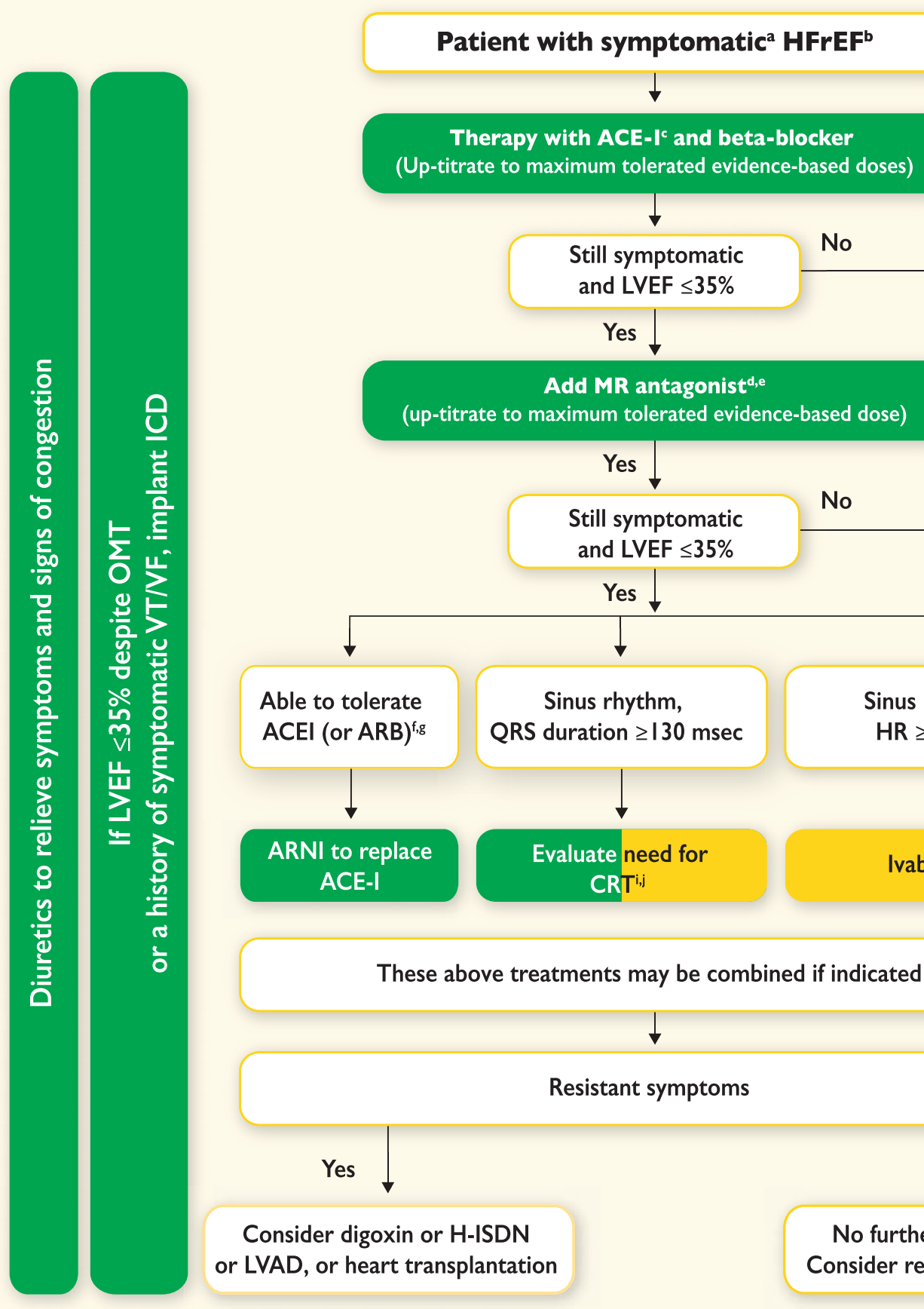

Class I

Class Ila

(Up-titrate to maximum tolerated evidence-based doses)

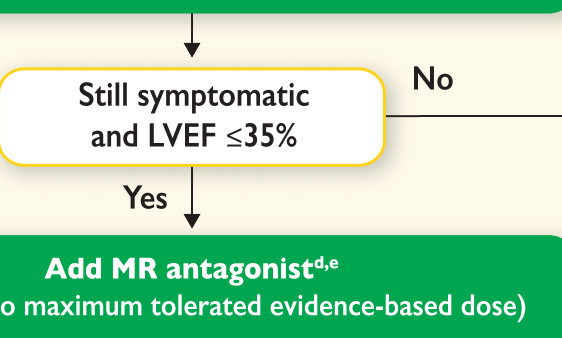

(up-titrate to maximum tolerated evidence-based dose)

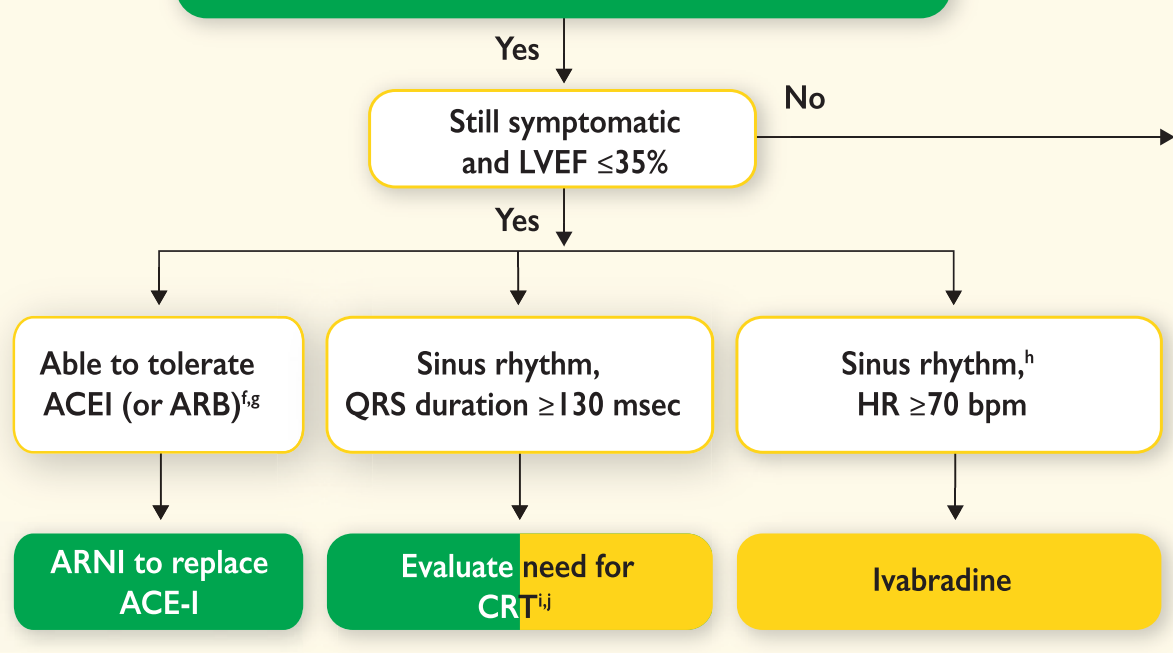

hese above treatments may be combined if indicated

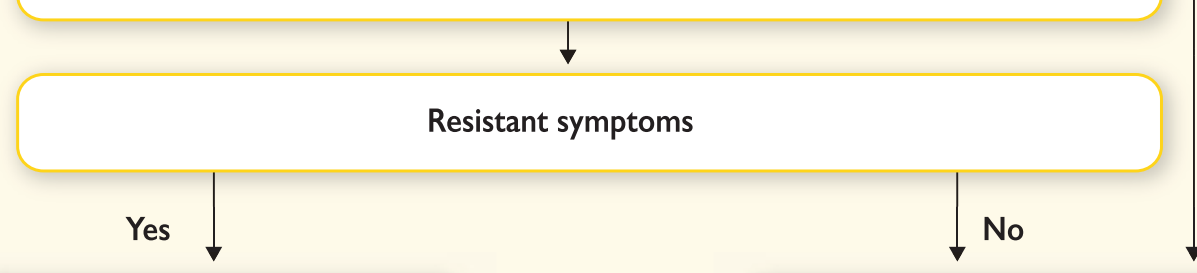

\section{Consider digoxin or $\mathrm{H}$-ISDN or LVAD, or heart transplantation}

No further action required Consider reducing diuretic dose

Figure 7.I Therapeutic algorithm for a patient with symptomatic heart failure with reduced ejection fraction. Green indicates a class I recommendation; yellow indicates a class lla recommendation. $\mathrm{ACEI}=$ angiotensin-converting enzyme inhibitor; $\mathrm{ARB}=$ angiotensin receptor blocker; $\mathrm{ARNI}=$ angiotensin receptor neprilysin inhibitor; $\mathrm{BNP}=\mathrm{B}$-type natriuretic peptide; $\mathrm{CRT}=$ cardiac resynchronization therapy; $\mathrm{HF}=$ heart failure; HFrEF = heart failure with reduced ejection fraction; $\mathrm{H}-\mathrm{ISDN}=$ hydralazine and isosorbide dinitrate; $\mathrm{HR}=$ heart rate; ICD = implantable cardioverter defibrillator; $L B B B=$ left bundle branch block; LVAD = left ventricular assist device; LVEF = left ventricular ejection fraction; MR = mineralocorticoid receptor; NT-proBNP = N-terminal pro-B type natriuretic peptide; NYHA = New York Heart Association; OMT = optimal medical therapy; VF = ventricular fibrillation; VT = ventricular tachycardia. ${ }^{a}$ Symptomatic $=$ NYHA Class II-IV. ${ }^{b} \mathrm{HFrEF}=\mathrm{LVEF}<40 \%$. ${ }^{\mathrm{C}}$ If $\mathrm{ACE}$

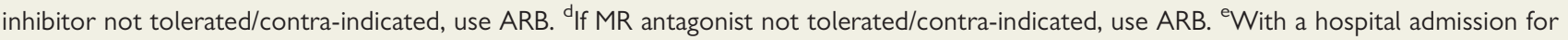
$\mathrm{HF}$ within the last 6 months or with elevated natriuretic peptides (BNP $>250 \mathrm{pg} / \mathrm{ml}$ or NTproBNP $>500 \mathrm{pg} / \mathrm{ml}$ in $\mathrm{men}$ and $750 \mathrm{pg} / \mathrm{ml}$ in women). 'With an elevated plasma natriuretic peptide level (BNP $\geq 150 \mathrm{pg} / \mathrm{mL}$ or plasma NT-proBNP $\geq 600 \mathrm{pg} / \mathrm{mL}$, or if HF hospitalization within recent 12 months plasma BNP $\geq 100 \mathrm{pg} / \mathrm{mL}$ or plasma NT-proBNP $\geq 400 \mathrm{pg} / \mathrm{mL}$ ). ${ }^{\text {g }} \mathrm{n}$ doses equivalent to enalapril $10 \mathrm{mg}$ b.i.d. ${ }^{\mathrm{h}} \mathrm{With}$ a hospital admission for HF within the previous year. ${ }^{i} \mathrm{CRT}$ is recommended if $\mathrm{QRS} \geq 130 \mathrm{msec}$ and LBBB (in sinus rhythm). ${ }^{\mathrm{j} C R T}$ should/may be considered if $\mathrm{QRS} \geq 130 \mathrm{msec}$ with non-LBBB (in a sinus rhythm) or for patients in AF provided a strategy to ensure bi-ventricular capture in place (individualized decision). For further details, see Sections 7 and 8 and corresponding web pages. 
mortality and morbidity have not been studied in RCTs. A Cochrane meta-analysis has shown that in patients with chronic HF, loop and thiazide diuretics appear to reduce the risk of death and worsening HF compared with placebo, and compared with an active control, diuretics appear to improve exercise capacity. $^{178,179}$

Table 7.2 Evidence-based doses of disease-modifying drugs in key randomized trials in heart failure with reduced ejection fraction (or after myocardial infarction)

\begin{tabular}{|c|c|c|}
\hline & Starting dose (mg) & Target dose (mg) \\
\hline \multicolumn{3}{|l|}{ ACE-I } \\
\hline Captopril ${ }^{a}$ & 6.25 ti.d. & 50 t.i.d. \\
\hline Enalapril & 2.5 b.i.d. & I0-20 b.i.d. \\
\hline Lisinopril $^{b}$ & $2.5-5.0$ o.d. & 20-35 o.d. \\
\hline Ramipril & 2.5 o.d. & 10 o.d. \\
\hline Trandolapril $^{a}$ & 0.5 o.d. & 4 o.d. \\
\hline \multicolumn{3}{|l|}{ Beta-blockers } \\
\hline Bisoprolol & I.25 o.d. & 10 o.d. \\
\hline Carvedilol & 3.125 b.i.d. & 25 b.i.d. ${ }^{d}$ \\
\hline Metoprolol succinate (CR/XL) & 12.5-25 o.d. & 200 o.d. \\
\hline Nebivololc $^{c}$ & I. 25 o.d. & 10 o.d. \\
\hline \multicolumn{3}{|l|}{ ARBs } \\
\hline Candesartan & 4-8 o.d. & 32 o.d. \\
\hline Valsartan & 40 b.i.d. & 160 b.i.d. \\
\hline Losartan ${ }^{b, c}$ & 50 o.d. & 150 o.d. \\
\hline \multicolumn{3}{|l|}{ MRAs } \\
\hline Eplerenone & 25 o.d. & 50 o.d. \\
\hline Spironolactone & 25 o.d. & 50 o.d. \\
\hline \multicolumn{3}{|l|}{ ARNI } \\
\hline Sacubitril/valsartan & 49/5I b.i.d. & 97/103 b.i.d. \\
\hline \multicolumn{3}{|l|}{ If-channel blocker } \\
\hline Ivabradine & 5 b.i.d. & 7.5 b.i.d. \\
\hline
\end{tabular}

$\mathrm{ACE}=$ angiotensin-converting enzyme; $\mathrm{ARB}=$ angiotensin receptor blocker; $\mathrm{ARNI}=$ angiotensin receptor neprilysin inhibitor; b.i.d. = bis in die (twice daily); $M R A=$ mineralocorticoid receptor antagonist; o.d. = omne in die (once daily); t.i.d. $=$ ter in die (three times a day).

andicates an ACE-I where the dosing target is derived from post-myocardial infarction trials.

bIndicates drugs where a higher dose has been shown to reduce morbidity/ mortality compared with a lower dose of the same drug, but there is no substantive randomized, placebo-controlled trial and the optimum dose is uncertain.

Indicates a treatment not shown to reduce cardiovascular or all-cause mortality in patients with heart failure (or shown to be non-inferior to a treatment that does).

${ }^{\mathrm{d}} \mathrm{A}$ maximum dose of $50 \mathrm{mg}$ twice daily can be administered to patients weighing over $85 \mathrm{~kg}$.
Loop diuretics produce a more intense and shorter diuresis than thiazides, although they act synergistically and the combination may be used to treat resistant oedema. However, adverse effects are more likely and these combinations should only be used with care. The aim of diuretic therapy is to achieve and maintain euvolaemia with the lowest achievable dose. The dose of the diuretic must be adjusted according to the individual needs over time. In selected asymptomatic euvolaemic/hypovolaemic patients, the use of a diuretic drug might be (temporarily) discontinued. Patients can be trained to self-adjust their diuretic dose based on monitoring of symptoms/signs of congestion and daily weight measurements.

Doses of diuretics commonly used to treat HF are provided in Table 7.3. Practical guidance on how to use diuretics is given in Web Table 7.7.

Table 7.3 Doses of diuretics commonly used in patients with heart failure

\begin{tabular}{|c|c|c|c|c|}
\hline Diuretics & \multicolumn{2}{|c|}{ Initial dose (mg) } & \multicolumn{2}{|c|}{$\begin{array}{l}\text { Usual daily dose } \\
\text { (mg) }\end{array}$} \\
\hline \multicolumn{5}{|l|}{ Loop diuretics $^{a}$} \\
\hline Furosemide & \multicolumn{2}{|l|}{$20-40$} & \multicolumn{2}{|l|}{$40-240$} \\
\hline Bumetanide & \multicolumn{2}{|l|}{$0.5-1.0$} & \multicolumn{2}{|l|}{$\mathrm{I}-5$} \\
\hline Torasemide & \multicolumn{2}{|l|}{$5-10$} & \multicolumn{2}{|l|}{$10-20$} \\
\hline \multicolumn{5}{|l|}{ Thiazides $^{\mathrm{b}}$} \\
\hline Bendroflumethiazide & \multicolumn{2}{|l|}{2.5} & \multicolumn{2}{|l|}{$2.5-10$} \\
\hline Hydrochlorothiazide & \multicolumn{2}{|l|}{25} & \multicolumn{2}{|l|}{$12.5-100$} \\
\hline Metolazone & \multicolumn{2}{|l|}{2.5} & \multicolumn{2}{|l|}{$2.5-10$} \\
\hline Indapamide $^{\mathrm{c}}$ & \multicolumn{2}{|l|}{2.5} & \multicolumn{2}{|l|}{$2.5-5$} \\
\hline \multicolumn{5}{|c|}{ Potassium-sparing diuretics ${ }^{d}$} \\
\hline & $\begin{array}{l}\text { +ACE-I/ } \\
\text { ARB }\end{array}$ & $\begin{array}{l}\text {-ACE-I/ } \\
\text { ARB }\end{array}$ & $\begin{array}{l}\text { +ACE-I/ } \\
\text { ARB }\end{array}$ & $\begin{array}{l}-A C E-I / \\
\text { ARB }\end{array}$ \\
\hline $\begin{array}{l}\text { Spironolactone/ } \\
\text { eplerenone }\end{array}$ & $12.5-25$ & 50 & 50 & $\begin{array}{l}100- \\
200\end{array}$ \\
\hline Amiloride & 2.5 & 5 & $5-10$ & $10-20$ \\
\hline Triamterene & 25 & 50 & 100 & 200 \\
\hline
\end{tabular}

ACE-I = angiontensin-converting enzyme inhibitor, $\mathrm{ARB}=$ angiotensin receptor blocker.

${ }^{a}$ Oral or intravenous; dose might need to be adjusted according to volume status/ weight; excessive doses may cause renal impairment and ototoxicity.

${ }^{b}$ Do not use thiazides if estimated glomerular filtration rate $<30 \mathrm{~mL} / \mathrm{min} / 1.73 \mathrm{~m}^{2}$, except when prescribed synergistically with loop diuretics.

'Indapamide is a non-thiazide sulfonamide.

${ }^{\mathrm{d}} \mathrm{A}$ mineralocorticoid antagonist (MRA) i.e. spironolactone/eplerenone is always preferred. Amiloride and triamterene should not be combined with an MRA. 


\section{Other pharmacological treatments recommended in selected patients with symptomatic (NYHA Class II-IV) heart failure with reduced ejection fraction}

\begin{tabular}{|c|c|c|c|}
\hline Recommendations & Class $^{a}$ & Level $^{\text {b }}$ & $\operatorname{Ref}^{c}$ \\
\hline \multicolumn{4}{|l|}{ Diuretics } \\
\hline Diuretics are recommended in order to improve symptoms and exercise capacity in patients with signs and/or symptoms of congestion. & I & B & 178,179 \\
\hline Diuretics should be considered to reduce the risk of HF hospitalization in patients with signs and/or symptoms of congestion. & Ila & B & 178,179 \\
\hline \multicolumn{4}{|l|}{ Angiotensin receptor neprilysin inhibitor } \\
\hline $\begin{array}{l}\text { Sacubitril/valsartan is recommended as a replacement for an ACE-I to further reduce the risk of HF hospitalization and death in } \\
\text { ambulatory patients with HFrEF who remain symptomatic despite optimal treatment with an ACE-I, a beta-blocker and an MRA }\end{array}$ & $\mathbf{I}$ & B & 162 \\
\hline \multicolumn{4}{|l|}{ If-channel inhibitor } \\
\hline $\begin{array}{l}\text { Ivabradine should be considered to reduce the risk of HF hospitalization or cardiovascular death in symptomatic patients } \\
\text { with LVEF } \leq 35 \% \text {, in sinus rhythm and a resting heart rate } \geq 70 \mathrm{bpm} \text { despite treatment with an evidence-based dose of beta- } \\
\text { blocker (or maximum tolerated dose below that),ACE-I (or ARB), and an MRA (or ARB). }\end{array}$ & Ila & $\mathbf{B}$ & 180 \\
\hline $\begin{array}{l}\text { Ivabradine should be considered to reduce the risk of } \mathrm{HF} \text { hospitalization and cardiovascular death in symptomatic patients with } \\
\mathrm{LVEF} \leq 35 \% \text {, in sinus rhythm and a resting heart rate } \geq 70 \mathrm{bpm} \text { who are unable to tolerate or have contra-indications for a } \\
\text { beta-blocker. Patients should also receive an ACE-I (or ARB) and an MRA (or ARB). }\end{array}$ & Ila & C & 181 \\
\hline \multicolumn{4}{|l|}{ ARB } \\
\hline $\begin{array}{l}\text { An ARB is recommended to reduce the risk of HF hospitalization and cardiovascular death in symptomatic patients unable to } \\
\text { tolerate an ACE-I (patients should also receive a beta-blocker and an MRA). }\end{array}$ & I & $\mathbf{B}$ & 182 \\
\hline $\begin{array}{l}\text { An ARB may be considered to reduce the risk of HF hospitalization and death in patients who are symptomatic despite treatment } \\
\text { with a beta-blocker who are unable to tolerate an MRA. }\end{array}$ & Ilb & C & - \\
\hline \multicolumn{4}{|l|}{ Hydralazine and isosorbide dinitrate } \\
\hline $\begin{array}{l}\text { Hydralazine and isosorbide dinitrate should be considered in self-identified black patients with LVEF } \leq 35 \% \text { or with an } \\
\text { LVEF }<45 \% \text { combined with a dilated LV in NYHA Class III-IV despite treatment with an ACE-I a beta-blocker and an MRA } \\
\text { to reduce the risk of HF hospitalization and death. }\end{array}$ & Ila & $\mathbf{B}$ & 183 \\
\hline $\begin{array}{l}\text { Hydralazine and isosorbide dinitrate may be considered in symptomatic patients with HFrEF who can tolerate neither an ACE-I } \\
\text { nor an ARB (or they are contra-indicated) to reduce the risk of death. }\end{array}$ & Ilb & $\mathbf{B}$ & 184 \\
\hline \multicolumn{4}{|l|}{ Digoxin } \\
\hline $\begin{array}{l}\text { Digoxin may be considered in symptomatic patients in sinus rhythm despite treatment with an ACE-I (or ARB), a beta-blocker } \\
\text { and an MRA, to reduce the risk of hospitalization (both all-cause and HF-hospitalizations). }\end{array}$ & IIb & B & 185 \\
\hline \multicolumn{4}{|l|}{ N-3 PUFA } \\
\hline $\begin{array}{l}\text { An n-3 PUFA }{ }^{e} \text { preparation may be considered in symptomatic HF patients to reduce the risk of cardiovascular hospitalization } \\
\text { and cardiovascular death. }\end{array}$ & Ilb & $\mathbf{B}$ & 186 \\
\hline
\end{tabular}

$\mathrm{ACEI}=$ angiotensin-converting enzyme inhibitor; $\mathrm{ARB}=$ angiotensin receptor blocker; $\mathrm{BNP}=\mathrm{B}$-type natriuretic peptide; bpm $=$ beats per minute; $\mathrm{HF}=$ heart failure; $\mathrm{HFrEF}=$ heart failure with reduced ejection fraction; LVEF = left ventricular ejection fraction; MRA = mineralocorticoid receptor antagonist; NT-proBNP = N-terminal pro-B type natriuretic peptide; NYHA = New York Heart Association; PUFA = polyunsaturated fatty acid. OMT = optimal medical therapy (for HFrEF this mostly comprises an ACEl or sacubitril/valsartan, a beta-blocker and an MRA).

${ }^{a}$ Class of recommendation.

bevel of evidence.

${ }^{\mathrm{c}}$ Reference(s) supporting recommendations.

${ }^{\mathrm{d}}$ Patient should have elevated natriuretic peptides (plasma BNP $\geq 150 \mathrm{pg} / \mathrm{mL}$ or plasma NT-proBNP $\geq 600 \mathrm{pg} / \mathrm{mL}$, or if HF hospitalization within the last $12 \mathrm{months}$, plasma BNP $\geq 100 \mathrm{pg} / \mathrm{mL}$ or plasma NT-proBNP $\geq 400 \mathrm{pg} / \mathrm{mL}$ ) and able to tolerate enalapril $10 \mathrm{mg}$ b.i.d.

${ }^{\mathrm{e}}$ Applies only to preparation studied in cited trial.

\subsubsection{Angiotensin receptor neprilysin inhibitor}

A new therapeutic class of agents acting on the RAAS and the neutral endopeptidase system has been developed [angiotensin receptor neprilysin inhibitor (ARNI)]. The first in class is LCZ696, which is a molecule that combines the moieties of valsartan and sacubitril (neprilysin inhibitor) in a single substance. By inhibiting neprilysin, the degradation of NPs, bradykinin and other peptides is slowed. High circulating A-type natriuretic peptide (ANP) and BNP exert physiologic effects through binding to NP receptors and the augmented generation of cGMP, thereby enhancing diuresis, natriuresis and myocardial relaxation and anti-remodelling. ANP and BNP also inhibit renin and aldosterone secretion. Selective AT1-receptor blockade reduces vasoconstriction, sodium and water retention and myocardial hypertrophy. ${ }^{187,188}$

A recent trial investigated the long-term effects of sacubitril/valsartan compared with an ACEI (enalapril) on morbidity 
and mortality in patients with ambulatory, symptomatic HFrEF with LVEF $\leq 40 \%$ (this was changed to $\leq 35 \%$ during the study), elevated plasma NP levels (BNP $\geq 150 \mathrm{pg} / \mathrm{mL}$ or NT-proBNP $\geq 600 \mathrm{pg} / \mathrm{mL}$ or, if they had been hospitalized for HF within the previous 12 months, BNP $\geq 100 \mathrm{pg} / \mathrm{mL}$ or NT-proBNP $\geq 400 \mathrm{pg} / \mathrm{mL}$ ), and an estimated GFR (eGFR) $\geq 30 \mathrm{~mL} / \mathrm{min} / 1.73 \mathrm{~m}^{2}$ of body surface area, who were able to tolerate separate treatments periods with enalapril (10 mg b.i.d.) and sacubitril/valsartan (97/103 mg b.i.d.) during a run-in period. ${ }^{162}$ In this population, sacubitril/valsartan (97/ $103 \mathrm{mg}$ b.i.d.) was superior to ACEI (enalapril $10 \mathrm{mg}$ b.i.d.) in reducing hospitalizations for worsening HF, cardiovascular mortality and overall mortality. ${ }^{162}$ Sacubitril/valsartan is therefore recommended in patients with HFrEF who fit this profile.

Despite the superiority of sacubitril/valsartan over enalapril in the PARADIGM-HF trial, some relevant safety issues remain when initiating therapy with this drug in clinical practice. Symptomatic hypotension was more often present in the sacubitril/ valsartan group (in those $\geq 75$ years of age, it affected $18 \%$ in the sacubitril/valsartan group vs. $12 \%$ in the enalapril group), although there was no increase in the rate of discontinuation. ${ }^{162}$ The risk of angioedema in the trial was reduced by recruiting only those who tolerated therapy with enalapril $10 \mathrm{mg}$ b.i.d. and an sacubitril/valsartan during an active run-in phase of 5-9 weeks (it resulted in a $0.4 \%$ rate of angioedema in sacubitril/valsartan group vs. $0.2 \%$ in an enalapril group). Also, the number of African American patients, who are at a higher risk of angioedema, was relatively small in this study. To minimize the risk of angioedema caused by overlapping ACE and neprilysin inhibition, the ACEl should be withheld for at least $36 \mathrm{~h}$ before initiating sacubitril/valsartan. Combined treatment with an ACEI (or ARB) and sacubitril/valsartan is contraindicated. There are additional concerns about its effects on the degradation of beta-amyloid peptide in the brain, which could theoretically accelerate amyloid deposition. ${ }^{189-191}$ However, a recent small 14-day study with healthy subjects showed elevation of the beta-amyloid protein in the soluble rather than the aggregable form, which if confirmed over longer time periods in patients with HFrEF may indicate the cerebral safety of sacubitril/valsar$\tan ^{192}$ Long-term safety needs to be addressed.

\subsubsection{If-channel inhibitor}

Ivabradine slows the heart rate through inhibition of the $I_{f}$ channel in the sinus node and therefore should only be used for patients in sinus rhythm. Ivabradine reduced the combined endpoint of mortality or hospitalization for HF in patients with symptomatic HFrEF or LVEF $\leq 35 \%$, in sinus rhythm and with a heart rate $\geq 70$ beats per minute (bpm) who had been hospitalized for HF within the previous 12 months, receiving treatment with an evidence-based dose of beta-blocker (or maximum tolerated dose), an ACEI (or ARB) and an MRA. ${ }^{180}$ The European Medicines Agency (EMA) approved ivabradine for use in Europe in patients with HFrEF with LVEF $\leq 35 \%$ and in sinus rhythm with a resting heart rate $\geq 75 \mathrm{bpm}$, because in this group ivabradine conferred a survival benefit ${ }^{193}$ based on a retrospective subgroup analysis requested by the EMA.
Practical guidance on how to use ivabradine is given in Web Table 7.8.

\subsubsection{Angiotensin II type I receptor blockers}

ARBs are recommended only as an alternative in patients intolerant of an ACEI. ${ }^{182}$ Candesartan has been shown to reduce cardiovascular mortality. ${ }^{182}$ Valsartan showed an effect on hospitalization for $\mathrm{HF}$ (but not on all-cause hospitalizations) in patients with $\mathrm{HFrEF}$ receiving background ACEls. ${ }^{194}$

The combination of ACEI/ARB for HFrEF was reviewed by the EMA, which suggested that benefits are thought to outweigh risks only in a select group of patients with HFrEF in whom other treatments are unsuitable. Therefore, ARBs are indicated for the treatment of HFrEF only in patients who cannot tolerate an ACEI because of serious side effects. The combination of ACEI/ARB should be restricted to symptomatic HFrEF patients receiving a beta-blocker who are unable to tolerate an MRA, and must be used under strict supervision.

\subsubsection{Combination of hydralazine and isosorbide dinitrate} There is no clear evidence to suggest the use of this fixed-dose combination therapy in all patients with HFrEF. Evidence on the clinical utility of this combination is scanty and comes from one relatively small RCT conducted exclusively in men and before ACEls or beta-blockers were used to treat HF. ${ }^{184}$ A subsequent RCT conducted in self-identified black patients (defined as being of African descent) showed that addition of the combination of hydralazine and isosorbide dinitrate to conventional therapy (ACEI, beta-blocker and MRA) reduced mortality and HF hospitalizations in patients with HFrEF and NYHA Classes III-IV. ${ }^{183}$ The results of this study are difficult to translate to patients of other racial or ethnic origins.

Additionally, a combination of hydralazine and isosorbide dinitrate may be considered in symptomatic patients with HFrEF who can tolerate neither ACEI nor ARB (or they are contraindicated) to reduce mortality. However, this recommendation is based on the results of the Veterans Administration Cooperative Study, which recruited symptomatic HFrEF patients who received only digoxin and diuretics. ${ }^{184}$

\subsection{Other treatments with less certain benefits in symptomatic patients with heart failure with reduced ejection fraction}

This section describes treatments that have shown benefits in terms of symptomatic improvement, reduction in HF hospitalizations or both, and are useful additional treatments in patients with HFrEF.

\subsubsection{Digoxin and other digitalis glycosides}

Digoxin may be considered in patients in sinus rhythm with symptomatic HFrEF to reduce the risk of hospitalization (both all-cause and HF hospitalizations), ${ }^{185}$ although its effect on top of betablockers has never been tested. The effects of digoxin in patients with HFrEF and AF have not been studied in RCTs, and recent studies have suggested potentially higher risk of events (mortality and HF 
hospitalization) in patients with AF receiving digoxin. ${ }^{195,196}$ However, this remains controversial, as another recent meta-analysis concluded on the basis of non-RCTs that digoxin has no deleterious effect on mortality in patients with AF and concomitant HF, most of whom had HFrEF. ${ }^{197}$

In patients with symptomatic HF and AF, digoxin may be useful to slow a rapid ventricular rate, but it is only recommended for the treatment of patients with HFrEF and AF with rapid ventricular rate when other therapeutic options cannot be pursued. ${ }^{196,198-201}$ Of note, the optimal ventricular rate for patients with HF and AF has not been well established, but the prevailing evidence suggests that strict rate control might be deleterious. A resting ventricular rate in the range of 70$90 \mathrm{bpm}$ is recommended based on current opinion, although one trial suggested that a resting ventricular rate of up to 110 bpm might still be acceptable. ${ }^{202}$ This should be tested and refined by further research.

Digitalis should always be prescribed under specialist supervision. Given its distribution and clearance, caution should be exerted in females, in the elderly and in patients with reduced renal function. In the latter patients, digitoxin should be preferred.

\subsection{2 $n-3$ polyunsaturated fatty acids}

$n-3$ polyunsaturated fatty acids ( $n-3$ PUFAs) have shown a small treatment effect in a large RCT. ${ }^{186} \mathrm{n}-3$ PUFA preparations differ in composition and dose. Only preparations with eicosapentaenoic acid (EPA) and docosahexaenoic acid (DHA) as ethyl esters of at least $85 \%(850 \mathrm{mg} / \mathrm{g})$ have shown an effect on the cumulative endpoint of cardiovascular death and hospitalization. No effect of n-3 PUFA preparations containing $<850 \mathrm{mg} / \mathrm{g}$ has been shown in either HFrEF or post-myocardial infarction. ${ }^{203} \mathrm{n}-3$ PUFA preparations containing $850-882 \mathrm{mg}$ of EPA and DHA as ethyl esters in the average ratio of $1: 1.2$ may be considered as an adjunctive therapy in patients with symptomatic HFrEF who are already receiving optimized recommended therapy with an ACEI (or ARB), a beta-blocker and an MRA.

\subsection{Treatments not recommended (unproven benefit) in symptomatic patients with heart failure with reduced ejection fraction}

\subsubsection{3-Hydroxy-3-methylglutaryl-coenzyme A reductase inhibitors ('statins')}

Although statins reduce mortality and morbidity in patients with atherosclerotic disease, statins are not effective in improving the prognosis in patients with HFrEF. Most statin trials excluded patients with HF (because it was uncertain that they would benefit). ${ }^{204}$ The two major trials that studied the effect of statin treatment in patients with chronic HF did not demonstrate any evidence of benefit. ${ }^{205}$ Therefore, evidence does not support the initiation of statins in most patients with chronic HF. However, in patients who already receive a statin because of underlying CAD or/and hyperlipidaemia, a continuation of this therapy should be considered.

\subsubsection{Oral anticoagulants and antiplatelet therapy}

Other than in patients with AF (both HFrEF and HFpEF), there is no evidence that an oral anticoagulant reduces mortality/morbidity compared with placebo or aspirin. ${ }^{206,207}$ Studies testing the nonvitamin $\mathrm{K}$ antagonist oral anticoagulants (NOACs) in patients with HFrEF are currently ongoing. Patients with HFrEF receiving oral anticoagulation because of concurrent AF or risk of venous thromboembolism should continue anticoagulation. Detailed information is provided in Section 10.1.

Similarly, there is no evidence on the benefits of antiplatelet drugs (including acetylsalicylic acid) in patients with HF without accompanying CAD, whereas there is a substantial risk of gastrointestinal bleeding, particularly in elderly subjects, related with this treatment.

\subsubsection{Renin inhibitors}

Aliskiren (direct renin inhibitor) failed to improve outcomes for patients hospitalized for HF at 6 months or 12 months in one study ${ }^{208}$ and is not presently recommended as an alternative to an ACEI or ARB.

Treatments (or combinations of treatments) that may cause harm in patients with symptomatic (NYHA Class II-IV) heart failure with reduced ejection fraction

\begin{tabular}{|l|c|c|c|}
\hline Recommendations & Class $^{\text {a }}$ & Level $^{\text {b }}$ & Ref $^{\text {c }}$ \\
\hline $\begin{array}{l}\text { Thiazolidinediones (glitazones) are } \\
\text { not recommended in patients with } \\
\text { HF, as they increase the risk of HF } \\
\text { worsening and HF hospitalization. }\end{array}$ & III & A & 209,210 \\
\hline $\begin{array}{l}\text { NSAIDs or COX-2 inhibitors are } \\
\text { not recommended in patients with } \\
\text { HF, as they increase the risk of HF } \\
\text { worsening and HF hospitalization. }\end{array}$ & III & B & 2 II- \\
\hline $\begin{array}{l}\text { Diltiazem or verapamil are not } \\
\text { recommended in patients with } \\
\text { HFrEF, as they increase the } \\
\text { risk of HF worsening and HF } \\
\text { hospitalization. }\end{array}$ & III & C & 214 \\
\hline $\begin{array}{l}\text { The addition of an ARB (or renin } \\
\text { inhibitor) to the combination } \\
\text { of an ACE-I and an MRA is not } \\
\text { recommended in patients with } \\
\text { HF, because of the increased } \\
\text { risk of renal dysfunction and } \\
\text { hyperkalaemia. }\end{array}$ & III & C & \\
\hline
\end{tabular}

$\mathrm{ACEI}=$ angiotensin-converting enzyme inhibitor; $\mathrm{ARB}=$ angiotensin recepto blocker; COX-2 inhibitor = cyclooxygenase-2 inhibitor; $\mathrm{HF}=$ heart failure; $\mathrm{HFrEF}=$ heart failure with reduced ejection fraction; $\mathrm{MRA}=$ mineralocorticoid receptor antagonist; NSAIDs $=$ non-steroidal anti-inflammatory drugs.

${ }^{\mathrm{a} C}$ Class of recommendation.

bLevel of evidence.

${ }^{\mathrm{c}}$ Reference(s) supporting recommendations 


\subsection{Treatments not recommended} (believed to cause harm) in symptomatic patients with heart failure with reduced ejection fraction

\subsubsection{Calcium-channel blockers}

Non-dihydropyridine calcium-channel blockers (CCBs) are not indicated for the treatment of patients with HFrEF. Diltiazem and verapamil have been shown to be unsafe in patients with HFrEF. ${ }^{214}$

There is a variety of dihydropyridine CCBs; some are known to increase sympathetic tone and they may have a negative safety profile in HFrEF. There is only evidence on safety for amlodipine ${ }^{215}$ and felodipine ${ }^{216}$ in patients with HFrEF, and they can be used only if there is a compelling indication in patients with HFrEF.

\section{Non-surgical device treatment of heart failure with reduced ejection fraction}

This section provides recommendations on the use of ICDs and CRT. Currently, the evidence is considered insufficient to support specific guideline recommendations for other therapeutic technologies, including baroreflex activation therapy, ${ }^{217}$ vagal stimulation, ${ }^{218}$ diaphragmatic pacing ${ }^{219,220}$ and cardiac contractility modulation; ${ }^{221,222}$ further research is required. Implantable devices to monitor arrhythmias or haemodynamics are discussed elsewhere in these guidelines.

\subsection{Implantable cardioverter-defibrillator}

A high proportion of deaths among patients with HF, especially those with milder symptoms, occur suddenly and unexpectedly. Many of these are due to electrical disturbances, including ventricular arrhythmias, bradycardia and asystole, although some are due to coronary, cerebral or aortic vascular events. Treatments that improve or delay the progression of cardiovascular disease will reduce the annual rate of sudden death, but they may have little effect on lifetime risk and will not treat arrhythmic events when they occur. ICDs are effective in preventing bradycardia and correcting potentially lethal ventricular arrhythmias. Some antiarrhythmic drugs might reduce the rate of tachyarrhythmias and sudden death, but they do not reduce overall mortality and may increase it.

Recommendations for implantable cardioverter-defibrillator in patients with heart failure

\begin{tabular}{|c|c|c|c|}
\hline Recommendations & Class $^{a}$ & Level $^{b}$ & $\operatorname{Ref}^{c}$ \\
\hline $\begin{array}{l}\text { Secondary prevention } \\
\text { An ICD is recommended to reduce the risk of sudden death and all-cause mortality in patients who have recovered from a } \\
\text { ventricular arrhythmia causing haemodynamic instability, and who are expected to survive for }>\text { I year with good functional status. }\end{array}$ & I & $\mathbf{A}$ & $223-226$ \\
\hline \multicolumn{4}{|l|}{$\begin{array}{l}\text { Primary prevention } \\
\text { An ICD is recommended to reduce the risk of sudden death and all-cause mortality in patients with symptomatic HF (NYHA } \\
\text { Class II-III), and an LVEF } \leq 35 \% \text { despite } \geq 3 \text { months of OMT, provided they are expected to survive substantially longer than one } \\
\text { year with good functional status, and they have: }\end{array}$} \\
\hline - IHD (unless they have had an $\mathrm{MI}$ in the prior 40 days - see below). & I & A & $\begin{array}{l}149,156, \\
227\end{array}$ \\
\hline - DCM. & I & $\mathbf{B}$ & $\begin{array}{l}156,157, \\
227\end{array}$ \\
\hline ICD implantation is not recommended within 40 days of an $\mathrm{Ml}$ as implantation at this time does not improve prognosis. & III & $\mathbf{A}$ & 158,228 \\
\hline $\begin{array}{l}\text { ICD therapy is not recommended in patients in NYHA Class IV with severe symptoms refractory to pharmacological therapy } \\
\text { unless they are candidates for CRT, a ventricular assist device, or cardiac transplantation. }\end{array}$ & III & C & $229-233$ \\
\hline $\begin{array}{l}\text { Patients should be carefully evaluated by an experienced cardiologist before generator replacement, because management goals } \\
\text { and the patient's needs and clinical status may have changed. }\end{array}$ & Ila & $\mathbf{B}$ & $234-238$ \\
\hline $\begin{array}{l}\text { A wearable ICD may be considered for patients with HF who are at risk of sudden cardiac death for a limited period or as a } \\
\text { bridge to an implanted device. }\end{array}$ & Ilb & C & 239-24I \\
\hline
\end{tabular}

CAD = coronary artery disease; $C R T=$ cardiac resynchronization therapy; DCM = dilated cardiomyopathy; HF = heart failure; ICD = implantable cardioverter-defibrillator; $\mathrm{IHD}=$ ischaemic heart disease; $\mathrm{LVEF}=$ left ventricular ejection fraction; MI = myocardial infarction; NYHA = New York Heart Association, OMT = optimal medical therapy.

${ }^{\mathrm{a}}$ Class of recommendation.

bLevel of evidence.

${ }^{c}$ Reference(s) supporting recommendations.

\subsubsection{Secondary prevention of sudden cardiac death}

Compared with amiodarone treatment, ICDs reduce mortality in survivors of cardiac arrest and in patients who have experienced sustained symptomatic ventricular arrhythmias. An ICD is recommended in such patients when the intent is to increase survival; the decision to implant should take into account the patient's view and their quality of life, the LVEF (survival benefit is uncertain when the LVEF is $>35 \%$ ) and the absence of other diseases likely to cause death within the following year. $^{223-225}$ 


\subsubsection{Primary prevention of sudden cardiac death}

Although amiodarone may have reduced mortality in older trials of $\mathrm{HF}^{242,243}$ contemporary studies conducted since the widespread introduction of beta-blockers suggest that it does not reduce mortality in patients with HFrEF. $227,244,245$ Dronedarone ${ }^{246,247}$ and class I antiarrhythmic agents ${ }^{246,248}$ should not be used for prevention of arrhythmias in this population.

Some guideline-recommended therapies, including betablockers, MRAs, sacubitril/valsartan and pacemakers with CRT (CRT-Ps), reduce the risk of sudden death (see Section 7).

An ICD reduces the rate of sudden arrhythmic death in patients with HFrEF. ${ }^{249,250}$ In patients with moderate or severe HF, a reduction in sudden death may be partially or wholly offset by an increase in death due to worsening $\mathrm{HF}^{227}$ In patients with mild HF (NYHA II), an ICD will prevent about two deaths per year for every 100 devices implanted. ${ }^{227}$ On average, patients with IHD are at greater risk of sudden death than patients with DCM and therefore, although the relative benefits are similar, the absolute benefit is greater in patients with IHD. ${ }^{249}$ Patients with longer QRS durations may also receive greater benefit from an ICD, but these patients should often receive a CRT device. ${ }^{227,251}$

Two RCTs showed no benefit in patients who had an ICD implanted within 40 days after a myocardial infarction. ${ }^{158,228} \mathrm{Al}$ though sudden arrhythmic deaths were reduced, this was balanced by an increase in non-arrhythmic deaths. Accordingly, an ICD is contraindicated in this time period. A wearable defibrillator may be considered if the patient is deemed to be at high risk of ventricular fibrillation, although evidence from randomized trials is lacking. ${ }^{239-241}$

ICD implantation is recommended only after a sufficient trial (minimum 3 months) of optimal medical therapy (OMT) has failed to increase the LVEF to $>35 \%$. However, one of the two landmark papers on which these recommendations are based included patients with an LVEF $>30 \%$. Fewer than 400 patients with an LVEF of $30-35 \%$ were included in the landmark studies, and although there was no statistical interaction between treatment effect and LVEF, the evidence of benefit is less robust in this group of patients.

Conservative programming with long delays ${ }^{252}$ between detection and the ICD delivering therapy dramatically reduces the risk of both inappropriate (due to artefacts or AF) and appropriate but unnecessary [due to self-terminating ventricular tachycardia (VT)] shocks. ${ }^{252-254}$

Patients with a QRS duration $\geq 130 \mathrm{~ms}$ should be considered for a defibrillator with CRT (CRT-D) rather than ICD. See the guideline on CRT for further details (Section 8.2).
ICD therapy is not recommended in patients in NYHA Class IV with severe symptoms refractory to pharmacological therapy who are not candidates for CRT, a ventricular assist device or cardiac transplantation, because such patients have a very limited life expectancy and are likely to die from pump failure.

Patients with serious co-morbidities who are unlikely to survive substantially more than 1 year are unlikely to obtain substantial benefit from an ICD. ${ }^{229-233}$

Patients should be counselled as to the purpose of an ICD, complications related to implantation and device activation (predominantly inappropriate shocks) and under what circumstances it might be deactivated (terminal disease) or explanted (infection, recovery of LV function). ${ }^{255}$

If HF deteriorates, deactivation of a patient's ICD may be considered after appropriate discussion with the patient and caregiver(s).

If the ICD generator reaches its end of life or requires explantation, it should not automatically be replaced. ${ }^{234-238}$ Patients should be carefully evaluated by an experienced cardiologist before generator replacement. Treatment goals may have changed and the risk of fatal arrhythmia may be lower or the risk of non-arrhythmic death higher. It is a matter of some controversy whether patients whose LVEF has greatly improved and who have not required device therapy during the lifetime of the ICD should have another device implanted. ${ }^{234-238}$

Subcutaneous defibrillators may be as effective as conventional ICDs with a lower risk from the implantation procedure. ${ }^{256,257}$ They may be the preferred option for patients with difficult access or who require ICD explantation due to infection. Patients must be carefully selected, as they have limited capacity to treat serious bradyarrhythmia and can deliver neither antitachycardia pacing nor CRT. Substantial RCTs with these devices and more data on safety and efficacy are awaited. ${ }^{258,259}$

A wearable ICD (an external defibrillator with leads and electrode pads attached to a wearable vest) that is able to recognize and interrupt VT/ventricular fibrillation may be considered for a limited period of time in selected patients with HF who are at high risk for sudden death but otherwise are not suitable for ICD implantation (e.g. those with poor LVEF after acute myocardial damage until LV function recovers, patients scheduled for heart transplantation). ${ }^{239-241,260}$ However, no prospective RCTs evaluating this device have been reported.

For detailed recommendations on the use/indications of ICD we refer the reader to the ESC/European Heart Rhythm Association (EHRA) guidelines on ventricular tachyarrhythmias and sudden cardiac death. ${ }^{260}$ 


\subsection{Cardiac resynchronization therapy}

Recommendations for cardiac resynchronization therapy implantation in patients with heart failure

\begin{tabular}{|c|c|c|c|}
\hline Recommendations & Class $^{\mathrm{a}}$ & Level $^{\mathrm{b}}$ & $\operatorname{Ref}^{c}$ \\
\hline $\begin{array}{l}\text { CRT is recommended for symptomatic patients with } \mathrm{HF} \text { in sinus rhythm with a QRS duration } \geq 150 \mathrm{msec} \text { and LBBB QRS } \\
\text { morphology and with LVEF } \leq 35 \% \text { despite OMT in order to improve symptoms and reduce morbidity and mortality. }\end{array}$ & $\mathbf{I}$ & $\mathbf{A}$ & $26 \mid-272$ \\
\hline $\begin{array}{l}\text { CRT should be considered for symptomatic patients with } \mathrm{HF} \text { in sinus rhythm with a QRS duration } \geq 150 \text { msec and non-LBBB } \\
\text { QRS morphology and with LVEF } \leq 35 \% \text { despite OMT in order to improve symptoms and reduce morbidity and mortality. }\end{array}$ & Ila & B & $26 \mid-272$ \\
\hline $\begin{array}{l}\text { CRT is recommended for symptomatic patients with HF in sinus rhythm with a QRS duration of I } 30-149 \text { msec and LBBB QRS } \\
\text { morphology and with LVEF } \leq 35 \% \text { despite OMT in order to improve symptoms and reduce morbidity and mortality. }\end{array}$ & $\mathbf{I}$ & B & 266,273 \\
\hline $\begin{array}{l}\text { CRT may be considered for symptomatic patients with HF in sinus rhythm with a QRS duration of I30-149 msec and non-LBBB } \\
\text { QRS morphology and with LVEF } \leq 35 \% \text { despite OMT in order to improve symptoms and reduce morbidity and mortality. }\end{array}$ & Illb & B & 266,273 \\
\hline $\begin{array}{l}\text { CRT rather than RV pacing is recommended for patients with HFrEF regardless of NYHA class who have an indication for ventricular } \\
\text { pacing and high degree AV block in order to reduce morbidity. This includes patients with AF (see Section 10.I). }\end{array}$ & $\mathbf{I}$ & A & 274-277 \\
\hline $\begin{array}{l}\text { CRT should be considered for patients with LVEF } \leq 35 \% \text { in NYHA Class III-IVd despite OMT in order to improve symptoms and } \\
\text { reduce morbidity and mortality, if they are in AF and have a QRS duration } \geq 130 \mathrm{msec} \text { provided a strategy to ensure bi-ventricular } \\
\text { capture is in place or the patient is expected to return to sinus rhythm. }\end{array}$ & Ila & B & $\begin{array}{c}275, \\
278-281\end{array}$ \\
\hline $\begin{array}{l}\text { Patients with HFrEF who have received a conventional pacemaker or an ICD and subsequently develop worsening HF despite OMT } \\
\text { and who have a high proportion of RV pacing may be considered for upgrade to CRT.This does not apply to patients with stable HF. }\end{array}$ & Ilb & B & 282 \\
\hline CRT is contra-indicated in patients with a QRS duration $<130 \mathrm{msec}$. & III & A & $\begin{array}{c}266, \\
283-285\end{array}$ \\
\hline $\begin{array}{l}\text { atrial fibrillation; } \mathrm{AV}=\text { atrio-ventricular; } \mathrm{CRT}=\text { cardiac resynchronization therapy; } \mathrm{HF}=\text { heart failure; } \mathrm{HFrEF}=\text { heart failure with } r \mathrm{r} \\
\text { ntable cardioverter-defibrillator; } \mathrm{LBBB}=\text { left bundle branch block; } \mathrm{LVEF}=\text { left ventricular ejection fraction; } \mathrm{NYHA}=\mathrm{New} \text { York Heart } \\
\text { py; } \mathrm{QRS}=\mathrm{Q}, \mathrm{R} \text { and } \mathrm{S} \text { waves (combination of three of the graphical deflections); } \mathrm{RV}=\text { right ventricular. } \\
\text { s of recommendation. } \\
\text { l of evidence. } \\
\text { rence(s) supporting recommendations. } \\
\text { judgement for patients with end-stage } \mathrm{HF} \text { who might be managed conservatively rather than with treatments to improve symptoms }\end{array}$ & uced $€$ & ion frac & $\begin{array}{l}n ; I C D= \\
\text { optimal m }\end{array}$ \\
\hline
\end{tabular}

CRT improves cardiac performance in appropriately selected patients and improves symptoms ${ }^{286}$ and well-being ${ }^{286}$ and reduces morbidity and mortality. ${ }^{266}$ Of the improvement in quality-adjusted life-years (QALYs) with CRT among patients with moderate to severe $\mathrm{HF}$, two-thirds may be attributed to improved quality of life and one-third to increased longevity. ${ }^{287}$

Only the COMPANION ${ }^{265}$ and CARE-HF trials ${ }^{262,263}$ compared the effect of CRT to guideline-advised medical therapy. Most other trials have compared CRT-D to ICD, and a few have compared CRT-P to backup pacing. The prevention of lethal bradycardia might be an important mechanism of benefit shared by all pacing devices. In CARE-HF, at baseline, $25 \%$ of patients had a resting heart rate of $\leq 60$ bpm. ${ }^{262-264}$ If prevention of bradycardia is important, the effect of CRT will appear greater in trials where there is no device in the control group.

Most studies of CRT have specified that the LVEF should be $<35 \%$, but RAFT ${ }^{267}$ and MADIT-CRT ${ }^{268,269}$ specified an LVEF $<30 \%$, while REVERSE ${ }^{270-272}$ specified $<40 \%$ and BLOCK-HF $F^{274}<50 \%$. Relatively few patients with an LVEF of $35-40 \%$ have been randomized, but an individual participant data (IPD) meta-analysis suggests no diminution of the effect of CRT in this group. ${ }^{266}$

Not all patients respond favourably to CRT. ${ }^{286}$ Several characteristics predict improvement in morbidity and mortality, and the extent of reverse remodelling is one of the most important mechanisms of action of CRT. Patients with ischaemic aetiology will have less improvement in LV function due to myocardial scar tissue, which is less likely to undergo favourable remodelling. ${ }^{288}$ Conversely, women may be more likely to respond than men, possibly due to smaller body and heart size. ${ }^{273,285,289}$ QRS width predicts
CRT response and was the inclusion criterion in all randomized trials. But QRS morphology has also been related to a beneficial response to CRT. Several studies have shown that patients with left bundle branch block (LBBB) morphology are more likely to respond favourably to CRT, whereas there is less certainty about patients with non-LBBB morphology. However, patients with LBBB morphology often have wider QRS duration, and there is a current debate about whether QRS duration or QRS morphology is the main predictor of a beneficial response to CRT. Evidence from two IPD meta-analyses indicates that after accounting for QRS duration, there is little evidence to suggest that QRS morphology or aetiology of disease influence the effect of CRT on morbidity or mortality. ${ }^{266,273}$ In addition, none of the landmark trials selected patients for inclusion according to QRS morphology, sex or ischaemic aetiology, nor were they powered for subgroup analyses.

The Echo-CRT ${ }^{283,284}$ trial and an IPD meta-analysis ${ }^{266}$ suggest possible harm from CRT when QRS duration is $<130 \mathrm{~ms}$, thus implantation of CRT is not recommended if QRS duration is $<130$ ms. $^{266,283,284}$

If a patient is scheduled to receive an ICD and is in sinus rhythm with a QRS duration $\geq 130 \mathrm{~ms}$, CRT-D should be considered if QRS is between 130 and $149 \mathrm{~ms}$ and is recommended if QRS is $\geq 150 \mathrm{~ms}$. However, if the primary reason for implanting a CRT is for the relief of symptoms, then the clinician should choose CRT-P or CRT-D, whichever they consider appropriate. Clinical practice varies widely among countries. The only randomized trial to compare CRT-P and CRT-D ${ }^{265}$ failed to demonstrate a difference in morbidity or mortality between these technologies. ${ }^{288}$ If the primary reason for implanting CRT is to improve prognosis, 
then the majority of evidence lies with CRT-D for patients in NYHA Class II and with CRT-P for patients in NYHA Classes III-IV. It is unclear whether CRT reduces the need for an ICD (by reducing the arrhythmia burden) or increases the benefit from an ICD (by reducing mortality rates from worsening HF, leading to longer exposure to the risk of arrhythmia).

When LVEF is reduced, RV pacing may exacerbate cardiac dyssynchrony. This can be prevented by CRT, which might improve patient outcomes. ${ }^{274,275,277,290}$ However, a difference in outcome was not observed between CRT and RV pacing in a subgroup analysis of RAFT $^{267}$ or in patients without HFrEF in BioPACE. ${ }^{291}$ On balance, CRT rather than $\mathrm{RV}$ pacing is recommended for patients with $\mathrm{HFrEF}$ regardless of NYHA class who have an indication for ventricular pacing in order to reduce morbidity, although no clear effect on mortality was observed. Patients with HFrEF who have received a conventional pacemaker or an ICD and subsequently develop worsening HF with a high proportion of RV pacing, despite OMT, may be considered for upgrading to CRT.

Only two small trials have compared pharmacological therapy alone vs. CRT in patients with AF, with conflicting results. Several studies have indicated that CRT is superior to RV pacing in patients undergoing atrio-ventricular (AV) node ablation. ${ }^{275,277,290}$ However, CRT is not an indication to carry out AV node ablation except in rare cases when ventricular rate remains persistently high $(>110$ bpm) despite attempts at pharmacological rate control. A subgroup analysis of patients with AF from the RAFT study found no benefit from CRT-D compared with ICD, although less than half of patients had $>90 \%$ biventricular capture. ${ }^{276}$ Observational studies report that when biventricular capture is $<98 \%$, the prognosis of patients with CRT declines. ${ }^{277}$ Whether this association reflects a loss of resynchronization (which might be remedied by device programming), poor placing of the LV lead (which might be avoided at implantation) or greater difficulty in pacing severely diseased myocardium (which might not be amenable to the above) is uncertain. This observation has not been confirmed in a randomized trial.

Imaging tests for dyssynchrony have not yet been shown to be of value in selecting patients for CRT. ${ }^{292}$ Patients with extensive myocardial scar will have less improvement in LV function with CRT, but this is true of any treatment for HFrEF and does not reliably predict less clinical benefit. ${ }^{293}$ Pacing thresholds are higher in scarred myocardium and, if possible, lead placement should avoid such regions. ${ }^{294,295}$ Although patients with extensive scarring have an intrinsically worse prognosis, there is little evidence that they obtain less prognostic benefit from CRT. ${ }^{266}$

The reader is directed to guidelines on pacing and CRT for recommendations on device implantation procedures. The value of trying to optimize AV or VV intervals after implantation using echo- or electrocardiographic criteria or blood pressure response is uncertain, but may be considered for patients who have had a disappointing response to CRT. ${ }^{296,297}$

\subsection{Other implantable electrical devices}

For patients with $\mathrm{HFrEF}$ who remain symptomatic despite OMT and do not have an indication for CRT, new device therapies have been proposed and in some cases are approved for clinical use in several European Union (EU) countries but remain under trial evaluation.

Cardiac contractility modulation (CCM) is similar in its mode of insertion to CRT, but it involves non-excitatory electrical stimulation of the ventricle during the absolute refractory period to enhance contractile performance without activating extra systolic contractions. CCM has been evaluated in patients with HFrEF in NYHA Classes II-III with normal QRS duration (<120 ms). ${ }^{221,222}$ An individual patient data meta-analysis demonstrated an improvement in exercise tolerance (peak $\mathrm{VO}_{2}$ ) and quality of life (Minnesota Living with Heart Failure questionnaire). Thus CCM may be considered in selected patients with HF. The effect of CCM on HF morbidity and mortality remains to be established.

Most other devices under evaluation involve some modification of the activity of the autonomic nervous system (ANS) by targeted electrical stimulation. ${ }^{298,299}$ These include vagal nerve stimulation, spinal cord stimulation, carotid body ablation and renal denervation, but so far none of the devices has improved symptoms or outcomes in RCTs.

Devices for remote monitoring are discussed in Section 14.

\section{Treatment of heart failure with preserved ejection fraction}

While there is clear agreement that the diagnosis of HFrEF requires an LVEF $<40 \%$, the exact definition of HFpEF is less clear. According to the definition provided in this document (see Section 3), the diagnosis of HFpEF requires an LVEF $\geq 50 \%$, whereas patients with LVEF between 40 and $49 \%$ are considered to have HFmrEF (for details, please refer to Section 3). Patients with HFmrEF have generally been included in trials of HFpEF. Accordingly, the guidance in this section applies to patients with both HFmrEF and HFpEF. As new data and analyses become available, it might be possible to make recommendations for each phenotype separately.

In clinical practice and clinical trials, compared with HFrEF patients, only slightly fewer patients with HFpEF and HFmrEF currently appear to receive diuretics, beta-blockers, MRAs and ACEls or ARBs. ${ }^{166,300-}$

302 This may reflect treatment of cardiovascular co-morbidities, such as hypertension, CAD and AF, or extrapolation of results from trials conducted for these conditions showing a reduction in new-onset $\mathrm{HF}^{127}$ or failure to distinguish between guideline recommendations for HFrEF and HFmrEF/HFpEF or a belief that existing clinical trials provide some evidence of benefit with these agents.

A summary of phase II and III clinical trials of patients with HFpEF and HFmrEF is presented in Web Table 9.1.

The pathophysiology underlying HFpEF and HFmrEF is heterogeneous, and they are associated with different phenotypes including diverse concomitant cardiovascular diseases (e.g. AF, arterial hypertension, CAD, pulmonary hypertension) and non-cardiovascular diseases [diabetes, chronic kidney disease (CKD), anaemia, iron deficiency, COPD and obesity]. ${ }^{303,304}$ Compared with HFrEF patients, hospitalizations and deaths in patients with HFmrEF/HFpEF are more likely to be non-cardiovascular. ${ }^{305,306}$ Therefore patients should be screened for cardiovascular and non-cardiovascular comorbidities, which if present should be managed with interventions that have been shown to improve symptoms, well-being or outcome related to that co-morbidity and not to exacerbate HF (see Section 11).

No treatment has yet been shown, convincingly, to reduce morbidity or mortality in patients with HFpEF or HFmrEF. However, since these patients are often elderly and highly symptomatic, and often have a poor quality of life, ${ }^{307}$ an important aim of therapy may be to alleviate symptoms and improve well-being. ${ }^{308}$ 


\subsection{Effect of treatment on symptoms in heart failure with preserved ejection fraction}

Diuretics will usually improve congestion, if present, thereby improving symptoms and signs of HF. The evidence that diuretics improve symptoms is similar across the spectrum of LVEF. ${ }^{178,179}$

Evidence that beta-blockers and MRAs improve symptoms in these patients is lacking. There is inconsistent evidence for an improvement in symptoms in those treated with ARBs (only for candesartan was there an improvement in NYHA class) ${ }^{309,310}$ and ACEls. ${ }^{311}$

\subsection{Effect of treatment on hospitalization for heart failure in heart failure with preserved ejection fraction}

For patients in sinus rhythm, there is some evidence that nebivolol, ${ }^{173,312,313}$ digoxin, ${ }^{314}$ spironolactone ${ }^{301}$ and candesartan ${ }^{310}$ might reduce HF hospitalizations. For patients in AF, beta-blockers do not appear to be effective and digoxin has not been studied. The evidence in support of either ARBs ${ }^{315}$ or ACEls ${ }^{311}$ is inconclusive.

\subsection{Effect of treatment on mortality in heart failure with preserved ejection fraction}

Trials of ACEls, ARBs, beta-blockers and MRAs have all failed to reduce mortality in patients with HFpEF or HFmrEF. However, in older patients with HFrEF, HFpEF or HFmrEF, nebivolol reduced the combined endpoint of death or cardiovascular hospitalization, ${ }^{173,312}$ with no significant interaction between treatment effect and baseline LVEF. ${ }^{313}$

\subsection{Other considerations}

Patients in AF should receive an anticoagulant to reduce the risk of thromboembolic events (for details, see the ESC guidelines of $\left.\mathrm{AF}^{316}\right]$. Antiplatelet agents are ineffective for this purpose. Renal dysfunction, which is common in this population, may contraindicate or increase the risk of haemorrhage with NOACs.

The optimal ventricular rate in patients with $\mathrm{HFmrEF/HFpEF} \mathrm{and}$ $\mathrm{AF}$ is uncertain, and aggressive rate control might be deleterious. Whether digoxin, beta-blockers or rate-limiting CCBs, or a combination of these, should be preferred is unknown. Verapamil or diltiazem should not be combined with a beta-blocker. There are insufficient data to recommend ablation strategies (either pulmonary venous or AV node) for HFpEF and HFmrEF.

Circumstantial evidence suggests that treating hypertension, often predominantly systolic, is important in HFmrEF/HFpEF. ${ }^{127,317}$ Diuretics, ACEls, ARBs and MRAs all appear appropriate agents, but beta-blockers may be less effective in reducing SBP. A recent study suggests that patients with hypertension and HFpEF or HFmrEF should not receive an ARB (olmesartan) if they are receiving ACEls and beta-blockers. ${ }^{318}$

The first-line oral hypoglycaemic drug for patients with HFpEF and HFmrEF should be metformin ${ }^{319}$ (see also Section 11.6). Recently, a trial of empagliflozin showed a reduction in blood pressure and body weight, probably by inducing glycosuria and osmotic diuresis. Its use was associated with a reduction in hospitalization for HF and in cardiovascular mortality. ${ }^{130}$ However, aggressive management of dysglycaemia may be harmful. ${ }^{153,320}$

Myocardial ischaemia may contribute to symptoms, morbidity and mortality and should be considered when assessing patients. However, there is only anecdotal evidence that revascularization improves symptoms or outcome. Patients with angina should follow the same management route as patients with HFrEF. ${ }^{112}$

Patients with HFpEF and HFmrEF have impaired exercise tolerance, commonly accompanied by an augmented blood pressure response to exercise and chronotropic incompetence. Combined endurance/resistance training appears safe for patients with HFpEF and HFmrEF and improves exercise capacity (as reflected by an increase in peak oxygen consumption), physical functioning score and diastolic function. ${ }^{307,321}$

\section{Recommendations for treatment of patients with heart failure with preserved ejection fraction and heart failure with mid-range ejection fraction}

\begin{tabular}{|l|c|c|c|}
\hline Recommendations & Class $^{\text {a }}$ & Level $^{\mathbf{b}}$ & Ref $^{\mathbf{c}}$ \\
\hline $\begin{array}{l}\text { it is recommended to screen } \\
\text { patients with HFpEF or HFmrEF } \\
\text { for both cardiovascular and non- } \\
\text { cardiovascular comorbidities, which, } \\
\text { if present, should be treated provided } \\
\text { safe and effective interventions exist } \\
\text { to improve symptoms, well-being } \\
\text { and/or prognosis. }\end{array}$ & I & C & \\
\hline $\begin{array}{l}\text { Diuretics are recommended in } \\
\text { congested patients with HFpEF } \\
\text { or HFmrEF in order to alleviate } \\
\text { symptoms and signs. }\end{array}$ & I & B & I78, I79 \\
\hline
\end{tabular}

$\mathrm{HFmrEF}=$ heart failure with mid-range ejection fraction; $\mathrm{HFpEF}=$ heart failure with preserved ejection fraction.

${ }^{a}$ Class of recommendation

bevel of evidence.

'Reference(s) supporting recommendations.

\section{Arrhythmias and conductance disturbances}

Ambulatory electrocardiographic monitoring can be used to investigate symptoms that may be due to arrhythmias, ${ }^{322-324}$ but evidence is lacking to support routine, systematic monitoring for all patients with HF to identify tachy- and bradyarrhythmias. There is no evidence that clinical decisions based on routine ambulatory electrocardiographic monitoring improve outcomes for patients with HF.

Ambulatory electrocardiographic recording detects premature ventricular complexes in virtually all patients with HF. Episodes of asymptomatic, non-sustained VT are common, increasing in frequency with the severity of $\mathrm{HF}$ and ventricular dysfunction and indicating a poor prognosis in patients with HF, but provide little discrimination between sudden death or death due to progressive $\mathrm{HF}^{316,325}$ Bradycardia and pauses are also commonly observed, especially at night when sympathetic activity is often lower and parasympathetic activity higher; sleep apnoea may be a trigger. ${ }^{326-328}$ Pauses are associated with a poor prognosis in patients with $C A D$ and left ventricular dysfunction. ${ }^{329}$ Bradyarrhythmias may make an important contribution to sudden death in $\mathrm{HF}^{330}$ 


\subsection{Atrial fibrillation}

$\mathrm{AF}$ is the most common arrhythmia in HF irrespective of concomitant LVEF; it increases the risk of thromboembolic complications (particularly stroke) and may impair cardiac function, leading to worsening symptoms of HF. ${ }^{316}$ Incident HF precipitated by AF is associated with a more benign prognosis, ${ }^{331}$ but new-onset $A F$ in a patient with established HF is associated with a worse outcome, probably because it is both a marker of a sicker patient and because it impairs cardiac function. ${ }^{332,333}$ Patients with chronic HF and permanent AF have a worse outcome than those in sinus rhythm, although this is largely explained by more advanced age and HF severity. ${ }^{332,333}$ Persistent ventricular rates $>150$ bpm may cause HFrEF that resolves with rate control or rhythm correction ('tachycardiomyopathy'). ${ }^{334,335}$ AF should be classified and managed according to the current AF guidelines (i.e. first diagnosed episode, paroxysmal, persistent, long-standing persistent or permanent), recognizing the uncertainty about the actual duration of the episode and about previous undetected episodes. ${ }^{316}$

The following issues need to be considered in patients with HF presenting with $A F$, irrespective of LVEF, especially with a first diagnosed episode of AF or paroxysmal AF: ${ }^{316}$

- identification of potentially correctable causes (e.g. hypothyroidism or hyperthyroidism, electrolyte disorders, uncontrolled hypertension, mitral valve disease) and precipitating factors (e.g. recent surgery, chest infection or exacerbation of COPD/ asthma, acute myocardial ischaemia, alcohol binge), as this may determine management strategy;

- assessment of stroke risk and need for anticoagulation;

- assessment of ventricular rate and need for rate control;

- evaluation of symptoms of HF and AF.

For details, the reader should refer to the 2016 ESC guidelines on AF. $^{316}$

\subsubsection{Prevention of atrial fibrillation in patients with heart failure}

Many treatments for $\mathrm{HF}$, including ACEls, ${ }^{336} \mathrm{ARBs},{ }^{337}$ betablockers ${ }^{177,338}$ and MRAs, ${ }^{339,340}$ will reduce the incidence of AF, but ivabradine may increase it. ${ }^{341}$ CRT has little effect on the incidence of $\mathrm{AF}^{342}$

Amiodarone will reduce the incidence of AF, induce pharmacological cardioversion, maintain more patients in sinus rhythm after cardioversion and may be used to control symptoms in patients with paroxysmal AF if beta-blockers fail to do so. ${ }^{343-346}$ Amiodarone should generally be restricted to short-term ( $<6$ months) use in patients with paroxysmal or persistent AF to help attain sinus rhythm and to reduce the high rate of recurrent AF immediately after cardioversion. Dronedarone is contraindicated in patients with $\mathrm{HF}$ and $\mathrm{AF}^{246,247,347}$

\subsubsection{Management of new-onset, rapid atrial fibrillation in patients with heart failure}

If the patient has no distressing symptoms of HF, then treatment with oral beta-blockers may be initiated to provide ventricular rate control. For patients with marked congestion who nonetheless have few symptoms at rest, initial treatment with oral or intravenous (i.v.) digoxin is preferred. For patients in haemodynamic instability, an i.v. bolus of digoxin or amiodarone ${ }^{348,349}$ should be administered into a peripheral vein with extreme care to avoid extravasation into tissues; where uncertainty exists about venous access, amiodarone must not be given. Longer-term infusion of amiodarone should be given only by central or long-line venous access to avoid peripheral vein phlebitis. In patients with haemodynamic collapse, emergency electrical cardioversion is recommended (see also Section 12).

\section{Recommendations for initial management of a rapid ventricular rate in patients with heart failure and atrial fibrillation in the acute or chronic setting}

\begin{tabular}{|c|c|c|c|}
\hline Recommendations & Class $^{a}$ & Level $^{b}$ & $\operatorname{Ref}^{c}$ \\
\hline $\begin{array}{l}\text { Urgent electrical cardioversion is } \\
\text { recommended if AF is thought to } \\
\text { be contributing to the patient's } \\
\text { haemodynamic compromise in } \\
\text { order to improve the patient clinical } \\
\text { condition. }\end{array}$ & I & C & \\
\hline $\begin{array}{l}\text { For patients in NYHA Class IV, in } \\
\text { addition to treatment for AHF, an } \\
\text { intravenous bolus of amiodarone } \\
\text { or, in digoxin-naïve patients, an } \\
\text { intravenous bolus of digoxin should } \\
\text { be considered to reduce the } \\
\text { ventricular rate. }\end{array}$ & Ila & B & 348,349 \\
\hline $\begin{array}{l}\text { For patients in NYHA Class I-III, a } \\
\text { beta-blocker, usually given orally, is } \\
\text { safe and therefore is recommended } \\
\text { as first-line treatment to control } \\
\text { ventricular rate, provided the patient } \\
\text { is euvolaemic. }\end{array}$ & I & $\mathbf{A}$ & 177 \\
\hline $\begin{array}{l}\text { For patients in NYHA Class I-III, } \\
\text { digoxin, should be considered } \\
\text { when ventricular rate remains high }{ }^{d} \\
\text { despite beta-blockers or when } \\
\text { beta-blockers are not tolerated or } \\
\text { contra-indicated. }\end{array}$ & Ila & $\mathbf{B}$ & 197 \\
\hline $\begin{array}{l}\text { AV node catheter ablation may be } \\
\text { considered to control heart rate } \\
\text { and relieve symptoms in patients } \\
\text { unresponsive or intolerant to } \\
\text { intensive pharmacological rate and } \\
\text { rhythm control therapy, accepting } \\
\text { that these patients will become } \\
\text { pacemaker dependent. }\end{array}$ & IIb & $\mathbf{B}$ & 290 \\
\hline $\begin{array}{l}\text { Treatment with dronedarone to } \\
\text { improve ventricular rate control } \\
\text { is not recommended due to safety } \\
\text { concerns. }\end{array}$ & III & $\mathbf{A}$ & 347 \\
\hline
\end{tabular}

$\mathrm{AF}=$ atrial fibrillation; $\mathrm{AHF}=$ acute heart failure; $\mathrm{AV}=$ atrio-ventricular; $\mathrm{bpm}=$ beats per minute; $\mathrm{HF}=$ heart failure; NYHA = New York Heart Association. ${ }^{a}$ Class of recommendation.

bLevel of evidence.

${ }^{\mathrm{C}}$ Reference(s) supporting recommendations.

${ }^{\mathrm{d}}$ The optimal ventricular rate for patients with $\mathrm{HF}$ and $\mathrm{AF}$ has not been established, but the prevailing evidence suggests that strict rate control might be deleterious. $A$ resting ventricular rate in the range of $60-100 \mathrm{bpm}$ may be considered based on the current opinion of this Task Force, ${ }^{350,351}$ although one trial suggested that a resting ventricular rate of up to $110 \mathrm{bpm}$ might still be acceptable, and this is currently recommended by the ESC guidelines on AF. ${ }^{198,316}$ This should be tested and refined by further research.

\subsubsection{Rate control}

Assessment of ventricular rate control from the radial pulse is not ideal, especially in patients with $\mathrm{HF}$, as ventricular activation may not always generate a palpable pulse. Rate control should be documented electrocardiographically. A wearable device enables 
ventricular rate to be assessed during rest, exercise and sleep, but the value of routine monitoring has not yet been established. Implanted devices such as pacemakers, CRT or ICDs can also be used to measure ventricular rate.

The optimal resting ventricular rate in patients with $\mathrm{AF}$ and $\mathrm{HF}$ is uncertain but may be between $60-100$ bpm. ${ }^{350,352-354}$ One trial suggested that a resting ventricular rate of up to $110 \mathrm{bpm}$ might still be acceptable, ${ }^{198,202}$ and 2016 ESC AF guidelines recommend this threshold as the target for rate control therapy. ${ }^{316}$ However, this Task Force believes that a lower rate for patients with HF may be preferable (60-100 bpm). Ventricular rates $<70$ bpm are associated with a worse outcome. ${ }^{351}$ This may explain why betablockers titrated to guideline-target doses failed to reduce morbidity or mortality in patients with $\mathrm{HFrEF}$ and $\mathrm{AF},{ }^{177}$ and might also explain the association between digoxin and adverse outcomes reported in some observational studies of $A F^{355-357}$ The optimal ventricular rate during exercise is also uncertain, but may be $<110$ bpm during light exercise. ${ }^{354}$ Beta-blockers, digoxin and their combination may be used to control ventricular rate. ${ }^{358} \mathrm{It}$ is uncertain which approach is optimal, but beta-blockers appear safe as the first-line agent even if it is not clear that they reduce morbidity and mortality in patients with AF. Beta-blockers reduce ventricular rate during periods of activity, while digoxin exerts a greater effect at night. ${ }^{358}$ Persistently high ventricular rates may indicate thyrotoxicosis or excessive sympathetic activity due to congestion, which might respond to diuresis. Although amiodarone and nondihydropyridine CCBs can reduce ventricular rate, they have more adverse effects and should generally be avoided in patients with HFrEF and, with less certainty, in patients with HFpEF and HFmrEF. Rarely, ventricular rate cannot be reduced below 100110 bpm by pharmacological means alone and AV node ablation with ventricular pacing may be considered; in this situation, for patients with HFrEF, CRT should be considered instead of conventional RV pacing. There is little evidence, other than from registries, to support a strategy of AV node ablation and CRT compared with pharmacological therapy alone in patients with $A F$ and a resting ventricular rate $<100-110 \mathrm{bpm}$ (see Section 8.2). ${ }^{281}$ However, in patients with a fast ventricular rate and intractable symptoms, AV node ablation may be considered. Also, if the patient is indicated for an ICD, AV node ablation with implantation of CRT-D may be a preferred option, especially if the patient has moderate to severe symptoms.

\subsubsection{Rhythm control}

In patients with chronic HF, a rhythm control strategy (including pharmacological or electrical cardioversion) has not been shown to be superior to a rate control strategy in reducing mortality or morbidity. ${ }^{359}$ Urgent cardioversion is indicated only if the AF is life threatening, otherwise both HF and ventricular rate should be controlled prior to cardioversion. A rhythm control strategy is probably best reserved for patients with a reversible secondary cause of AF (e.g. hyperthyroidism) or an obvious precipitant (e.g. recent pneumonia) and in patients with troublesome symptoms due to AF after optimization of rate control and HF therapy. The use of class I antiarrhythmic agents and dronedarone increases morbidity and mortality in patients with $\mathrm{HF}$ and $\mathrm{AF}$ and should be avoided. ${ }^{246,247,347}$ Amiodarone will cause some patients with chronic AF to revert to sinus rhythm, may reduce symptomatic paroxysms of $A F$ and will help maintain patients in sinus rhythm after spontaneous or electrical cardioversion. ${ }^{343-346}$ When used, the need for continued administration of amiodarone should be regularly reviewed and justified.

The safety and efficacy of catheter ablation in the atria and pulmonary veins (PV) as a rhythm control strategy in HF is at present uncertain except for tachycardia induced cardiomyopathy. ${ }^{316}$ One small study suggested that AF ablation was superior to AV node ablation and CRT. ${ }^{360}$ Another study, including 203 patients with persistent AF, HF and an ICD or CRT device, showed that AF ablation was superior to amiodarone in correcting AF, and this was associated with fewer hospitalizations for HF and lower mortality. Two small studies of AF ablation compared with rate control met with mixed success in terms of procedural complications and success in improving symptoms. ${ }^{278,279}$ The most recent evidence from a meta-analysis that included 914 patients suggests an encouraging success rate of $P V$ ablation of $A F$ in patients with LV dysfunction, with improvements in LVEF and functional capacity. ${ }^{361}$ These results need to be confirmed in ongoing RCTs such as CASTLE $A F,{ }^{362}$ AMICA and CABANA.

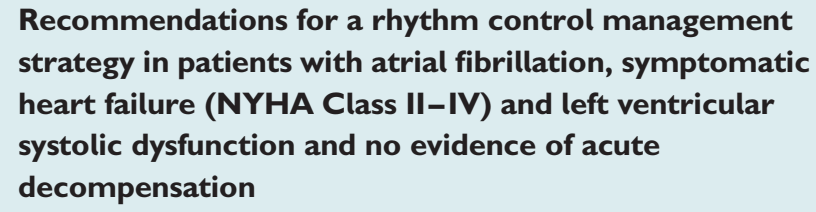

\begin{tabular}{|c|c|c|c|}
\hline Recommendations & Class $^{a}$ & Level $^{\mathrm{b}}$ & $\operatorname{Ref}^{c}$ \\
\hline $\begin{array}{l}\text { Electrical cardioversion or } \\
\text { pharmacological cardioversion with } \\
\text { amiodarone may be considered in } \\
\text { patients with persisting symptoms } \\
\text { and/or signs of HF, despite OMT and } \\
\text { adequate control of ventricular rate, } \\
\text { to improve clinical/symptomatic } \\
\text { status. }\end{array}$ & Ilb & B & 344 \\
\hline $\begin{array}{l}\text { AF ablation may be considered in } \\
\text { order to restore sinus rhythm to } \\
\text { improve symptoms in patients with } \\
\text { persisting symptoms and/or signs } \\
\text { of HF, despite OMT and adequate } \\
\text { control of ventricular rate, to } \\
\text { improve clinical/symptomatic status. }\end{array}$ & Ilb & B & 279,363 \\
\hline $\begin{array}{l}\text { Amiodarone may be considered } \\
\text { prior to (and following) successful } \\
\text { electrical cardioversion to maintain } \\
\text { sinus rhythm. }\end{array}$ & Ilb & B & 342,360 \\
\hline $\begin{array}{l}\text { Dronedarone is not recommended } \\
\text { because of an increased risk } \\
\text { of hospital admissions for } \\
\text { cardiovascular causes and an } \\
\text { increased risk of premature death in } \\
\text { NYHA Class III-IV patients. }\end{array}$ & III & $\mathbf{A}$ & 247,347 \\
\hline $\begin{array}{l}\text { Class I antiarrhythmic agents are } \\
\text { not recommended because of an } \\
\text { increased risk of premature death. }\end{array}$ & III & A & $\begin{array}{c}248,364 \\
365\end{array}$ \\
\hline
\end{tabular}

$\mathrm{AF}=$ atrial fibrillation; $\mathrm{HF}=$ heart failure; $\mathrm{NYHA}=$ New York Heart Association, OMT $=$ optimal medical therapy.

Patients should generally be anticoagulated for 6 weeks prior to electrical cardioversion.

${ }^{\mathrm{a}}$ Class of recommendation.

bLevel of evidence.

${ }^{\mathrm{C}}$ Reference(s) supporting recommendations. 


\subsubsection{Thromboembolism prophylaxis}

Patients with $\mathrm{HF}$ and $\mathrm{AF}$ should generally be anticoagulated and the balance of benefit and risk of bleeding (using $\mathrm{CHA}_{2} \mathrm{DS}_{2}$-VASc and HAS-BLED scores; for details, please see Web Tables 10.1 and 10.2.) should be evaluated as recommended in the ESC guidelines for AF. ${ }^{316}$ A substantial proportion of patients with HF will have both benefit and risk scores $\geq 3$, indicating that careful consideration should be given before prescribing an oral anticoagulant and that regular review is subsequently needed (and correctable risk factors for bleeding addressed) if an oral anticoagulant is given.

NOACs are preferred for patients with $\mathrm{HF}$ with non-valvular AF, as NOACs compared with vitamin $\mathrm{K}$ antagonists seem to be at least similarly effective and even safer (less intracranial haemorrhage) in patients with $\mathrm{HF}$ than in subjects without $\mathrm{HF},{ }^{316,366,367}$ although concerns exist about their safety in older patients with $\mathrm{HF}$ and poor renal function ${ }^{368,369}$ [for a detailed description of the interaction between NOAC and renal function, see Heidbuchel et al. $\left.{ }^{370}\right]$. In patients with
$\mathrm{HF}$ and $\mathrm{AF}$ who have mechanical heart valves or at least moderate mitral stenosis, only oral vitamin $\mathrm{K}$ antagonists should be used for prevention of thromboembolic stroke. ${ }^{370}$

The dabigatran dose should be reduced to $110 \mathrm{mg}$ b.i.d. when creatinine clearance is $30-49 \mathrm{~mL} / \mathrm{min}$, rivaroxaban to $15 \mathrm{mg}$ daily and edoxaban to $30 \mathrm{mg}$ daily when creatinine clearance is $30-50 \mathrm{~mL} /$ min and apixaban to $2.5 \mathrm{mg}$ twice daily if a patient has two or more of the following: age $\geq 80$ years, serum creatinine $\geq 1.5 \mathrm{mg} /$ $\mathrm{dL}$ or body weight $\leq 60 \mathrm{~kg}$. ${ }^{370-375}$ The summary of the recommendations for the prevention of thromboembolism in patients with symptomatic HF and paroxysmal or persistent/permanent $A F$ is presented in the recommendations table. For further details, please refer to the recent ESC guidelines on $\mathrm{AF}^{316}$

A left atrial occlusion device could be considered in a patient with AF as an alternative to an oral anticoagulant who is at high-risk both of thromboembolism and of bleeding in order to avoid the risk of haemorrhage due to anticoagulation risk. ${ }^{381,382}$

\section{Recommendations for the prevention of thrombo-embolism in patients with symptomatic heart failure (NYHA Class II- IV) and paroxysmal or persistent/permanent atrial fibrillation}

\begin{tabular}{|c|c|c|c|}
\hline Recommendations & Class $^{a}$ & Level $^{b}$ & $\operatorname{Ref}^{c}$ \\
\hline $\begin{array}{l}\text { The } \mathrm{CHA}_{2} \mathrm{DS}_{2}-\mathrm{VASc} \text { and HAS-BLED scores are recommended tools in patients with HF for the estimation of the risk of } \\
\text { thromboembolism and the risk of bleeding associated with oral anticoagulation, respectively. }\end{array}$ & I & $\mathbf{B}$ & 376,377 \\
\hline $\begin{array}{l}\text { An oral anticoagulant is recommended to prevent thrombo-embolism for all patients with paroxysmal or persistent/permanent } \\
A F \text { and a } \mathrm{CHA}_{2} \mathrm{DS}_{2} \text {-VASc score } \geq 2 \text {, without contra-indications, and irrespective of whether a rate or rhythm management strategy } \\
\text { is used (including after successful cardioversion). }\end{array}$ & I & $\mathbf{A}$ & $\begin{array}{l}372-375 \\
378,379\end{array}$ \\
\hline $\begin{array}{l}\text { In patients with } A F \text { of } \geq 48 \text { h duration, or when the duration of } A F \text { is unknown, an oral anticoagulant is recommended at a } \\
\text { therapeutic dose for } \geq 3 \text { weeks prior to electrical or pharmacological cardioversion. }\end{array}$ & $\mathbf{I}$ & $\mathbf{B}$ & \\
\hline $\begin{array}{l}\text { Intravenous heparin or } \mathrm{LMWH} \text { and TOE quided strategy is recommended for patients who have not been treated with an } \\
\text { anticoagulant dose for } \geq 3 \text { weeks and require urgent electrical or pharmacological cardioversion for a life threatening arrhythmia. }\end{array}$ & $\mathbf{I}$ & C & \\
\hline $\begin{array}{l}\text { Combination of an oral anticoagulant and an antiplatelet agent is not recommended in patients with chronic ( }>12 \text { months } \\
\text { after an acute event) coronary or other arterial disease, because of a high-risk of serious bleeding. Single therapy with an oral } \\
\text { anticoagulant is preferred after } 12 \text { months. }\end{array}$ & III & C & \\
\hline $\begin{array}{l}\text { For patients with } \mathrm{HF} \text { and non-valvular } \mathrm{AF} \text { eligible for anticoagulation based on a } \mathrm{CHA}_{2} \mathrm{DS}_{2} \text {-VASc score, NOACs rather than } \\
\text { warfarin should be considered for anticoagulation as NOACs are associated with a lower risk of stroke, intracranial haemorrhage } \\
\text { and mortality, which outweigh the increased risk of gastrointestinal haemorrhage. }\end{array}$ & Ila & $\mathbf{B}$ & 367 \\
\hline
\end{tabular}

$\mathrm{AF}=$ atrial fibrillation; $\mathrm{CHA}_{2} \mathrm{DS}_{2}-\mathrm{VASc}=$ Congestive heart failure or left ventricular dysfunction, Hypertension, Age $\geq 75$ (doubled), Diabetes, Stroke (doubled)-Vascular disease, Age 65-74, Sex category (female); HAS-BLED = Hypertension, Abnormal renal/liver function, Stroke, Bleeding history or predisposition, Labile international normalized ratio, Elderly (>65 years), Drugs/alcohol concomitantly (1 point each); HF = heart failure; LMWH = low molecular weight heparin; NOAC = non-vitamin K antagonist oral anticoagulant; NYHA = New York Heart Association; TOE = transoesophageal echocardiography.

${ }^{a}$ Class of recommendation.

'Level of evidence.

${ }^{\mathrm{C}}$ Reference(s) supporting recommendations.

\subsection{Ventricular arrhythmias}

The initial management of asymptomatic ventricular arrhythmias is correction of electrolyte abnormalities, particularly low serum potassium and magnesium, withdrawal of agents that might provoke arrhythmias and, in patients with HFrEF, optimization of pharmacological therapy with ACEls, beta-blockers and MRAs and sacubitril/valsartan, which all reduce the risk of sudden death. ${ }^{174,177,383,384}$

The clinical relevance of myocardial ischaemia for the provocation of ventricular arrhythmias is uncertain, although anecdotal cases of ischaemia-induced arrhythmias exist. Randomized trials of revascularization for patients with HFrEF have not reduced overall mortality, ${ }^{107,385}$ even in subgroups of patients with angina or myocardial ischaemia, ${ }^{115,386}$ but further analysis did suggest a reduction in sudden deaths. ${ }^{387}$

Amiodarone (often in combination with a beta-blocker) may be used to suppress symptomatic ventricular arrhythmias, but it may adversely affect prognosis, especially in patients with more severe HF. ${ }^{227,244}$ Other antiarrhythmic drugs should be avoided. ${ }^{247}$ Transcatheter radiofrequency modification of the arrhythmogenic substrate may reduce the number of appropriate ICD discharges and may be used to terminate arrhythmic storm in patients with 
$\mathrm{HF}$ and frequent, recurrent ventricular tachyarrhythmias and therefore should be considered in such patients. Seeking the advice of the members of the HF Team with expertise in electrophysiology is recommended in patients with recalcitrant ventricular arrhythmias. For further details we refer the reader to the ESC/ EHRA guidelines on ventricular arrhythmias and sudden cardiac death. $^{260}$

\section{Recommendations for the management of ventricular tachyarrhythmias in heart failure}

\begin{tabular}{|c|c|c|c|}
\hline Recommendations & Class $^{a}$ & Level $^{\text {b }}$ & $\operatorname{Ref}^{c}$ \\
\hline $\begin{array}{l}\text { Potential aggravating/precipitating } \\
\text { factors (e.g. low serum potassium/ } \\
\text { magnesium, ongoing ischaemia) } \\
\text { should be sought and corrected in } \\
\text { patients with ventricular arrhythmias. }\end{array}$ & Ila & C & \\
\hline $\begin{array}{l}\text { Treatment with beta-blocker, MRA } \\
\text { and sacubitril/valsartan reduces } \\
\text { the risk of sudden death and is } \\
\text { recommended for patients with } \\
\text { HFrEF and ventricular arrhythmias } \\
\text { (as for other patients)(see Section 7). }\end{array}$ & I & $\mathbf{A}$ & $\begin{array}{c}162 \\
170-175\end{array}$ \\
\hline $\begin{array}{l}\text { Implantation of an ICD or CRT-D } \\
\text { device is recommended for selected } \\
\text { patients with HFrEF (see Section 8). }\end{array}$ & I & $\mathbf{A}$ & $\begin{array}{c}223-226 \\
388\end{array}$ \\
\hline $\begin{array}{l}\text { Several strategies should be } \\
\text { considered to reduce } \\
\text { recurrent symptomatic arrhythmias } \\
\text { in patients with an ICD } \\
\text { (or in those who are not eligible } \\
\text { for ICD), including attention to risk } \\
\text { factors and optimal pharmacological } \\
\text { treatment of HF, amiodarone, } \\
\text { catheter ablation and CRT. }\end{array}$ & Ila & C & \\
\hline $\begin{array}{l}\text { Routine use of antiarrhythmic } \\
\text { agents is not recommended in } \\
\text { patients with HF and asymptomatic } \\
\text { ventricular arrhythmias because } \\
\text { of safety concerns (worsening HF, } \\
\text { proarrhythmia, and death). }\end{array}$ & III & $\mathbf{A}$ & $\begin{array}{l}247,248 \\
364,365\end{array}$ \\
\hline
\end{tabular}

ACEI = angiotensin-converting enzyme inhibitor; $A R B=$ angiotensin receptor blocker; CRT = cardiac resynchronization therapy; CRT-D = defibrillator with cardiac resynchronization therapy; $\mathrm{HF}=$ heart failure; $\mathrm{HFrEF}=$ heart failure with reduced ejection fraction; ICD = implantable cardioverter defibrillator; $M R A=$ mineralocorticoid receptor antagonist.

${ }^{\mathrm{a}}$ Class of recommendation.

bLevel of evidence.

${ }^{c}$ Reference(s) supporting recommendations.

\subsection{Symptomatic bradycardia, pauses and atrio-ventricular block}

The ESC Guidelines on Pacing and CRT recommended intervention when pauses exceed $6 \mathrm{~s}$, even when this is not associated with symptoms. ${ }^{389}$ However, these recommendations were generated mainly for patients without obvious myocardial dysfunction, and shorter pauses might require intervention in patients with HFrEF. ${ }^{329}$ If pauses $>3$ s are identified on electrocardiographic monitoring, medications should be reviewed and the following agents stopped or reduced in dose, starting with rate-limiting $\mathrm{CCBs}$ then amiodarone, digoxin and ivabradine. For patients in $\mathrm{AF}$, a reduction in the dose of beta-blockers allowing the daytime resting ventricular rate to rise to $70-90 \mathrm{bpm}$ may be considered, since evidence that beta-blockers improve outcome in patients with AF is lacking. ${ }^{177}$ For patients with pauses but in sinus rhythm, a reduction in the dose of beta-blockers should be avoided unless the pauses are symptomatic, prolonged or frequent, in which case the relative merits of dose reduction, beta-blocker withdrawal and (biventricular) pacing may be considered. However, evidence is lacking to support a strategy of pacing solely to permit initiation or titration of beta-blocker therapy in the absence of a conventional pacing indication; this strategy is not recommended. For patients with HFrEF and high-degree AV block, CRT is preferred over RV pacing (Section 8.2). When the cause of bradycardia or pauses is sinus node disease with intact AV conduction, then therapeutic strategies that avoid inducing ventricular dyssynchrony are preferred, although clinical trial evidence to support this expert opinion for patients with HF is sparse. For other pacing indications, please consult the ESC Guidelines on Pacing and CRT. $^{389}$

\section{Recommendations for the management of bradyarrhythmias in heart failure}

\begin{tabular}{|c|c|c|c|}
\hline Recommendations & Class $^{a}$ & Level $^{\text {b }}$ & $\operatorname{Ref}^{c}$ \\
\hline $\begin{array}{l}\text { When pauses }>3 \text { seconds are } \\
\text { identified on the ECG, or if the } \\
\text { bradycardia is symptomatic and the } \\
\text { resting ventricular rate is }<50 \mathrm{bpm} \\
\text { in sinus rhythm or }<60 \mathrm{bpm} \text { in } \mathrm{AF} \text {, } \\
\text { it should be considered whether } \\
\text { there is need for any rate limiting } \\
\text { medications prescribed; for patients } \\
\text { in sinus rhythm beta-blockers should } \\
\text { be reduced in dose or withdrawn } \\
\text { only as a last resort. }\end{array}$ & Ila & C & \\
\hline $\begin{array}{l}\text { For patients with symptomatic, } \\
\text { prolonged or frequent pauses } \\
\text { despite adjustment of rate limiting } \\
\text { medication, either beta-blocker } \\
\text { withdrawal or pacing may be } \\
\text { considered as the next step. }\end{array}$ & Ilb & C & \\
\hline $\begin{array}{l}\text { Pacing solely to permit initiation or } \\
\text { titration of beta-blocker therapy in } \\
\text { the absence of a conventional pacing } \\
\text { indication is not recommended. }\end{array}$ & III & C & \\
\hline $\begin{array}{l}\text { In patients with HFrEF who require } \\
\text { pacing and who have high degree } \mathrm{AV} \\
\text { block, CRT rather than RV pacing is } \\
\text { recommended. }\end{array}$ & I & A & $\begin{array}{c}274,275 \\
290\end{array}$ \\
\hline $\begin{array}{l}\text { In patients with HFrEF who require } \\
\text { pacing who do not have high degree } \\
\text { AV block, pacing modes that avoid } \\
\text { inducing or exacerbating ventricular } \\
\text { dyssynchrony should be considered. }\end{array}$ & Ila & C & \\
\hline
\end{tabular}

$\mathrm{AF}=$ atrial fibrillation; $\mathrm{AV}=$ atrio-ventricular; $\mathrm{bpm}=$ beats per minute; $\mathrm{CRT}=$ cardiac resynchronization therapy; ECG = electrocardiogram; HFrEF = heart failure with reduced ejection fraction; $\mathrm{RV}=$ right ventricular.

${ }^{a}$ Class of recommendation.

bevel of evidence.

${ }^{\mathrm{c}}$ Reference(s) supporting recommendations. 


\section{Co-morbidities}

\subsection{Heart failure and co-morbidities}

Co-morbidities are of great importance in HF (Table 11.1) and may affect the use of treatments for HF (e.g. it may not be possible to use renin-angiotensin system inhibitors is some patients with severe renal dysfunction) (see Section 7). The drugs used to treat comorbidities may cause worsening of HF (e.g. NSAIDs given for arthritis, some anti-cancer drugs) (see Section 7). Management of comorbidities is a key component of the holistic care of patients with HF (see Section 14). Many co-morbidities are actively managed by specialists in the field of the co-morbidity, and these physicians will follow their own specialist guidelines. The current guidelines will identify where the presence of HF should change the way a comorbidity would normally be treated. This may be because either safety or efficacy may be different in the presence of HF (or may simply be unknown) or because of evidence of particular effects in an HF population, either beneficial or detrimental. HFpEF has an even higher prevalence of co-morbidities compared with HFrEF, and many of these may be instrumental in the progression of this syndrome. $^{398}$

\subsection{Angina and coronary artery disease} 11.2.1 Pharmacological management

Beta-blockers, and in selected patients ivabradine, ${ }^{180}$ are effective agents for angina control, as well as an essential component of HFrEF therapy. In HFpEF patients, they may also be used for angina relief, although this has never been formally tested. ${ }^{399}$

Trimetazidine has been shown to exert some beneficial effect as an add-on to beta-blockers in patients with HF and angina. ${ }^{400-406}$ There are data suggesting that it may improve NYHA functional capacity, exercise duration and LV function in patients with HFrEF. ${ }^{402-406}$ Certain other effective anti-anginal drugs have been studied in sizeable numbers of HFrEF/LV dysfunction patients and shown to be safe [e.g. amlodipine, ${ }^{215,407}$ nicorandil ${ }^{408}$ and nitrates $\left.{ }^{183,184,409}\right]$. The safety of other anti-anginal agents in HFrEF, such as ranolazine, is uncertain, while other drugs, specifically diltiazem and verapamil, are thought to be unsafe in patients with HFrEF (although they may be used in HFpEF). ${ }^{214}$ Dihydropyridine CCBs may all increase sympathetic tone, and their safety in HFrEF [except amlodipine ${ }^{215}$ and felodipine ${ }^{216}$ ] and HFpEF is uncertain.

\subsubsection{Myocardial revascularization}

For indications for invasive coronary angiography in patients with HF, please refer to Section 5.8.

Percutaneous and surgical revascularization are complementary approaches for symptomatic relief of angina in HFpEF, but whether these interventions improve outcomes is not entirely clear. Recent ESC guidelines on myocardial revascularization recommended coronary artery bypass grafting (CABG) for patients with significant left main stenosis and left main equivalent (proximal stenosis of both the left anterior descending and left circumflex arteries) to
Table II.I Importance of co-morbidities in patients with heart failure

\begin{tabular}{|c|}
\hline $\begin{array}{l}\text { I. interfere with the diagnostic process of HF (e.g. COPD as a } \\
\text { potentially confounding cause of dyspnoea). }{ }^{390,391}\end{array}$ \\
\hline 2. aggravate HF symptoms and further impair quality of life. ${ }^{391,392}$ \\
\hline $\begin{array}{l}\text { 3. contribute to the burden of hospitalizations and mortality, }{ }^{393} \text { as the } \\
\text { main cause of readmissions at I and } 3 \text { months. }{ }^{394}\end{array}$ \\
\hline $\begin{array}{l}\text { 4. may affect the use of treatments for HF (e.g. renin-angiotensin } \\
\text { system inhibitors contra-indicated in some patients with severe renal } \\
\text { dysfunction or beta-blockers relatively contra-indicated in asthma). }{ }^{395,396}\end{array}$ \\
\hline $\begin{array}{l}\text { 5. evidence base for HF treatment is more limited as co-morbidities were } \\
\text { mostly an exclusion criterion in trials; efficacy and safety of interventions } \\
\text { is therefore often lacking in the presence of co-morbidities. }\end{array}$ \\
\hline $\begin{array}{l}\text { 6. drugs used to treat co-morbidities may cause worsening HF (e.g. } \\
\text { NSAIDs given for arthritis, some anti-cancer drugs). }{ }^{397}\end{array}$ \\
\hline $\begin{array}{l}\text { 7. interaction between drugs used to treat HF and those used to treat } \\
\text { co-morbidities, resulting in lower efficacy, poorer safety, and the } \\
\text { occurrence of side effects (e.g. beta-blockers for HFrEF and beta- } \\
\text { agonists for COPD and asthma). }{ }^{391,395,396}\end{array}$ \\
\hline
\end{tabular}

$\mathrm{HF}=$ heart failure; $\mathrm{COPD}=$ chronic obstructive pulmonary disease; $\mathrm{HFrEF}=$ heart failure with reduced ejection fraction; NSAIDs = non-steroidal anti-inflammatory drugs.

improve prognosis. ${ }^{112,113}$ However, one needs to be aware of a lack of studies including patients who have well-defined HF, therefore this recommendation is solely based on expert opinion. On the basis of the results of the STICH trial [which excluded patients with left main disease and Canadian Cardiovascular Society (CCS) angina classes III-IV], CABG is also recommended in patients with HFrEF, significant CAD (left anterior descending artery or multivessel disease) and LVEF $\leq 35 \%$ to reduce death and hospitalization for cardiovascular causes. ${ }^{385}$ Patients with $>10 \%$ dysfunctional but viable LV myocardium may be more likely to benefit from myocardial revascularization (and those with $\leq 10 \%$ are less likely to benefit), although this approach to patient selection for revascularization is unproven. In the STICH trial, neither the presence of viability nor the severity of LV remodelling identified those who benefited from CABG in terms of a reduction in mortality. ${ }^{118}$ For the assessment of techniques to assess myocardial viability, please refer to Section 5. Post hoc analyses from the STICH trial revealed that the presence of inducible myocardial ischaemia (either on radionuclide stress test or dobutamine stress echocardiogram) or angina does not identify those with worse prognosis and greater benefit from CABG over OMT. ${ }^{115,386}$ However, CABG does improve angina to a greater extent than medical therapy alone.

The choice between CABG and PCl should be made by the Heart Team after careful evaluation of the patient's clinical status and coronary anatomy, expected completeness of revascularization, coexisting valvular disease and co-morbidities. 
Recommendations for the treatment of stable angina pectoris with symptomatic (NYHA Class II-IV) heart failure with reduced ejection fraction ${ }^{112,113}$

\begin{tabular}{|c|c|c|c|}
\hline Recommendations & Class $^{a}$ & Level $^{\text {b }}$ & $\operatorname{Ref}^{c}$ \\
\hline \multicolumn{4}{|l|}{ Step I } \\
\hline $\begin{array}{l}\text { A beta-blocker (in an evidence-based dose or maximum tolerated) is recommended as the preferred first-line treatment to relieve } \\
\text { angina because of the associated benefits of this treatment (reducing the risk of HF hospitalization and the risk of premature death). }\end{array}$ & I & $\mathbf{A}$ & $167-173$ \\
\hline \multicolumn{4}{|l|}{ Step 2: on top of beta-blocker or if a beta-blocker is not tolerated } \\
\hline $\begin{array}{l}\text { lvabradine should be considered as an anti-anginal drug in suitable HFrEF patients (sinus rhythm and HR } \geq 70 \mathrm{bpm} \text { ) as per } \\
\text { recommended HFrEF management. }\end{array}$ & Ila & B & $\begin{array}{l}180,410, \\
411\end{array}$ \\
\hline \multicolumn{4}{|l|}{ Step 3: For additional angina symptom relief - except from any combination not recommended } \\
\hline A short-acting oral or transcutaneous nitrate should be considered (effective anti-anginal treatment, safe in HF). & Ila & $\mathbf{A}$ & $\begin{array}{l}183,184, \\
409\end{array}$ \\
\hline A long acting oral or transcutaneous nitrate should be considered (effective anti-anginal treatment, not extensively studied in HF). & Ila & B & 183,184 \\
\hline $\begin{array}{l}\text { Trimetazidine may be considered when angina persists despite treatment with a beta-blocker (or alternative) to relieve angina } \\
\text { (effective anti-anginal treatment, safe in HF). }\end{array}$ & IIb & A & $400-403$ \\
\hline Amlodipine may be considered in patients unable to tolerate a beta-blocker to relieve angina (effective anti-anginal treatment, safe in HF). & Illb & B & 215,407 \\
\hline $\begin{array}{l}\text { Nicorandil may be considered in patients unable to tolerate a beta-blocker to relieve angina (effective anti-anginal treatment, } \\
\text { but safety in HF uncertain). }\end{array}$ & Ilb & C & \\
\hline $\begin{array}{l}\text { Ranolazine may be considered in patients unable to tolerate a beta-blocker to relieve angina (effective anti-anginal treatment, but } \\
\text { safety in HF uncertain). }\end{array}$ & Ilb & C & \\
\hline \multicolumn{4}{|l|}{ Step 4: Myocardial revascularization } \\
\hline Myocardial revascularization is recommended when angina persists despite treatment with anti-angina drugs. & I & A & $\begin{array}{l}385,412 \\
413\end{array}$ \\
\hline $\begin{array}{l}\text { Alternatives to myocardial revascularization: combination of } \geq 3 \text { antianginal drugs (from those listed above) may be considered } \\
\text { when angina persists despite treatment with beta-blocker, ivabradine and an extra anti-angina drug (excluding the combinations } \\
\text { not recommended below). }\end{array}$ & Ilb & C & \\
\hline \multicolumn{4}{|l|}{ The following are NOT recommended: } \\
\hline (2) Combination of nicorandil and a nitrate (because of lack of additional efficacy). & III & C & \\
\hline Diltiazem and verapamil are not recommended because of their negative inotropic action and risk of worsening $\mathrm{HF}$. & III & C & 214 \\
\hline
\end{tabular}

bpm = beats per minute; $\mathrm{HF}=$ heart failure; $\mathrm{HFrEF}=$ heart failure with reduced ejection fraction; NYHA = New York Heart Association.

${ }^{a}$ Class of recommendation.

'Level of evidence.

${ }^{\mathrm{c}}$ Reference(s) supporting recommendations.

\subsection{Cachexia and sarcopenia (for frailty, please refer to Section 14)}

Cachexia is a generalized wasting process affecting all body compartments [i.e. lean tissue (skeletal muscle), fat tissue (energy reserves) and bone tissue (osteoporosis)]. It may occur in 5-15\% of patients with $\mathrm{HF}$, especially those with $\mathrm{HFrEF}$, and more advanced disease status. ${ }^{414-416}$ This serious complication is associated with more severe symptoms and reduced functional capacity, more frequent hospitalization and decreased survival. Cachexia in HF can be diagnosed and defined as involuntary non-oedematous weight loss $\geq 6 \%$ of total body weight within the previous 6-12 months. ${ }^{414-417}$

The causes are multifactorial, and in individual patients they are difficult to determine. These may include pro-inflammatory immune activation, neurohormonal derangements, poor nutrition and malabsorption, impaired calorie and protein balance, anabolic hormone resistance, reduced anabolic drive, prolonged immobilization and physical deconditioning, together characterized by catabolic/anabolic imbalance. ${ }^{418}$ Skeletal muscle wasting, when associated with impaired mobility and symptoms (termed sarcopenia or myopenia), occurs in $30-50 \%$ of patients with HFrEF. ${ }^{419}$ In its most severe form it is associated with frailty and poor morbidity and mortality. ${ }^{420}$

Potential treatments may include appetite stimulants, exercise training ${ }^{120}$ and anabolic agents, including testosterone, in combination with the application of nutritional supplements and anti-catabolic interventions, although none is of proven benefit and their safety is unknown. ${ }^{421}$

\subsection{Cancer}

Certain chemotherapeutic agents can cause (or aggravate) LV systolic dysfunction and HF. The best recognized of these are the 
anthracyclines (e.g. doxorubicin), trastuzumab and tyrosine kinase inhibitors. ${ }^{397,422}$ A recent Cochrane review found that dexrazoxane may confer some cardioprotection in patients receiving anthracyclines. ${ }^{423}$ Pre- and post-evaluation of LVEF, if available with myocardial strain imaging, is essential in patients receiving cardiotoxic chemotherapy, as detailed elsewhere. ${ }^{397,422}$ A risk score for identifying women with breast cancer at risk of developing HF during trastuzumab therapy has been developed based on age, chemotherapy details, baseline cardiovascular status and other co-morbidities, and may be helpful. ${ }^{424}$ Chemotherapy should be discontinued and HFrEF therapy commenced in patients developing moderate to severe $L V$ systolic dysfunction. If $L V$ function improves, the risks and benefits of further chemotherapy need to be reconsidered. ${ }^{397,425,426}$ Mediastinal irradiation can also lead to a variety of long-term cardiac complications. Cardiac biomarkers (NPs and troponins) can be used to identify patients at higher risk of cardiotoxicity and may be helpful in monitoring the use and dosing of cardiotoxic cytotoxics. ${ }^{397,425,426}$

\subsection{Central nervous system (including depression, stroke and autonomic dysfunction)}

Stroke and HF commonly coexist because of an overlap of shared risk factors. Both contribute to a worse prognosis. Stroke may make self-care more difficult for the HF patient. Management of high-risk stroke patients may require balancing the risk of anticoagulant and antiplatelet therapies.

Autonomic dysfunction is common in HFrEF, especially when severe. ${ }^{427}$ Combined with low blood pressure, it can make fainting and injuries more likely and can interfere with optimal dosing of betablockers, ACEls, ARBs and MRAs. Diuretic dosage may be reduced to reduce the severity of postural hypotension.

Depression is common and is associated with worse clinical status and a poor prognosis in HF. ${ }^{428-430}$ It may also contribute to poor adherence and social isolation. A high index of suspicion is needed to make the diagnosis, especially in the elderly. Routine screening using a validated questionnaire is good practice. Until now, the Beck Depression Inventory (BDI) and Cardiac Depression Scale have been formally validated as reliable tools for the assessment of depressive mood in patients with $\mathrm{HF},{ }^{431,432}$ but other questionnaires have been broadly used in this group of patients (e.g. Geriatric Depression Scale, Hamilton Depression Scale, Hospital Anxiety and Depression Scale).

Psychosocial intervention and pharmacological treatment are helpful, as well as exercise training, in patients with HFrEF and depression. ${ }^{433}$ Cognitive behavioural therapy delivered in patients with HF and major depression beyond standard care and a structured education programme were able to reduce depression severity, anxiety and fatigue symptoms, as well as improve social functioning and mental and HF-related quality of life. $^{434}$

Selective serotonin reuptake inhibitors are thought to be safe, although the Sertraline Antidepressant Heart Attack Randomized Trial did not confirm that sertraline provides a greater reduction in depressive symptoms or improvement in cardiovascular status compared with placebo in HFrEF patients, but this trial was not powered enough to prove the latter. ${ }^{435}$ Similarly, escitalopram had no effect on either depression or clinical outcomes during the 24-month follow-up as compared with placebo in patients with HFrEF and depression. Importantly, tricyclic antidepressants should be avoided, because they may cause hypotension, worsening HF and arrhythmias. $^{429,435}$

\subsection{Diabetes}

Dysglycaemia and diabetes are very common in HF, and diabetes is associated with poorer functional status and worse prognosis. In patients with HFrEF, interventions that reduce morbidity and mortality confer similar benefit in the presence or absence of diabetes. ${ }^{320}$ For instance, beta-blockers improve outcome similarly, whether or not the patient has diabetes, although different beta-blockers may vary in their effects on glycaemic indices. ${ }^{436}$

Whether strict glycaemic control alters the risk of cardiovascular events in patients with $\mathrm{HF}$ is uncertain. ${ }^{437}$ Among patients with $\mathrm{HF}$ who have not been treated for diabetes, higher $\mathrm{HbA} 1 \mathrm{c}$ is associated with greater risk of cardiovascular events, ${ }^{438,439}$ but this may not be the case once treatment for diabetes has been commenced. ${ }^{439}$

In patients with diabetes and HF, glycaemic control should be implemented gradually and moderately, giving preference to those drugs, such as metformin, that have been shown to be safe and effective. In contrast to what was previously believed, metformin is safe to use in patients with HFrEF, and it should be the treatment of choice in patients with $\mathrm{HF}^{440,441}$ but is contraindicated in patients with severe renal or hepatic impairment, because of the risk of lactic acidosis.

Insulin is required for patients with type 1 diabetes and to treat symptomatic hyperglycaemia in patients with type 2 diabetes and pancreatic islet $\beta$ cell exhaustion. However, insulin is a powerful sodium-retaining hormone, and when combined with a reduction in glycosuria, may exacerbate fluid retention, leading to HF worsening. Sulphonylurea derivatives have also been associated with an increased risk of worsening HF and should be used with caution.

Thiazolidinediones (glitazones) cause sodium and water retention and increased risk of worsening $\mathrm{HF}$ and hospitalization and are not recommended in patients with HF. ${ }^{209,210}$ Dipeptidylpeptidase-4 inhibitors (DPP4is; gliptins), which increase incretin secretion, thereby stimulating insulin release, and longacting glucagon-like peptide 1 (GLP-1) receptor agonists, which act as incretin mimetics, improve glycaemic indices but do not reduce and may increase the risk of cardiovascular events and worsening HF. ${ }^{320,442,443}$ Importantly, there are no data on the safety of gliptins and GLP-1 analogues in patients with HF.

Recently, empagliflozin, an inhibitor of sodium-glucose cotransporter 2, reduced hospitalization for HF and mortality, but not myocardial infarction or stroke, in patients with diabetes at high cardiovascular risk, some of whom had HF. ${ }^{130}$ In the absence of other studies with drugs from this group, the results obtained with empaglifozin cannot be considered as a proof of a class effect. 
As glycaemic derangement progresses, the judgement on glycaemic control should be made according to cardiac conditions, and if the new anti-diabetic drugs are to be prescribed, they have to be closely monitored by an HF team.

\subsection{Erectile dysfunction}

Erectile dysfunction is a common and important component of quality of life in men with $\mathrm{HF}^{444,445}$ Its treatment should include optimal therapies for underlying cardiovascular diseases and other interfering co-morbidities (e.g. diabetes) and amelioration of anxiety and depressive symptoms. Some drugs applied for HF therapy (e.g. thiazide diuretics, spironolactone and beta-blockers) may augment erectile dysfunction. ${ }^{444,445}$ Phosphodiesterase type 5 inhibitors (PDE5Is) have been shown to have favourable haemodynamic and anti-remodelling effects and to improve exercise capacity and quality of life in patients with $\mathrm{HFrEF},{ }^{446,447}$ but they are contraindicated in patients taking nitrates.

\subsection{Gout and arthritis}

Hyperuricaemia and gout are common in HF and may be caused or aggravated by diuretic treatment. Hyperuricaemia is associated with a worse prognosis in HFrEF. ${ }^{448}$ The current European League Against Rheumatism (EULAR) guideline for the management of gout recommends that urate-lowering therapy (ULT) is indicated in patients with recurrent acute flares, arthropathy, tophi or radiographic changes of gout, aiming to maintain a serum urate level below the saturation point for monosodium urate $[<357 \mu \mathrm{mol} / \mathrm{L}$ $(<6 \mathrm{mg} / \mathrm{dL})]^{449}$

Xanthine oxidase inhibitors (allopurinol, oxypurinol) may be used to prevent gout, although their safety in HFrEF is uncertain. ${ }^{450}$ Gout attacks are better treated with colchicine rather than with NSAIDs (although colchicine should not be used in patients with very severe renal dysfunction and may cause diarrhoea). Intra-articular corticosteroids are an alternative for monoarticular gout, but systemic corticosteroids cause sodium and water retention.

Arthritis is a common co-morbidity and is a common cause of both self-taken and prescribed drugs that can worsen renal function and HF, especially NSAIDs. Rheumatoid arthritis is associated with an increased risk of HFpEF. The safety of disease-modifying drugs commonly given to patients with rheumatoid arthritis has not been established in HF.

\subsection{Hypokalaemia and hyperkalaemia}

Both hypokalaemia and hyperkalaemia are associated with $\mathrm{HF}$ and with many drugs used for HF treatment. ${ }^{451}$ Both can aggravate ventricular arrhythmias.

Loop and thiazide diuretics reduce serum potassium, while ACEls, ARBs and MRAs can all increase serum potassium. Amiloride and triamterene are sometimes used as adjunct diuretics in resistant oedema and to assist in preventing hypokalaemia. The treatment of hypokalaemia can involve recommending high potassium foods or prescribing potassium supplements.
The management of acute hyperkalaemia ( $>6.0 \mathrm{mmol} / \mathrm{L})$ may require a short-term cessation of potassium-retaining agents and RAAS inhibitors, but this should be minimized and RAAS inhibitors should be carefully reintroduced as soon as possible while monitoring potassium levels. A Cochrane review ${ }^{452}$ found no trial evidence of major outcome benefits for any emergency therapy regimen for hyperkalaemia. Two new potassium binders (patiromer and sodium zirconium cyclosilicate) are currently under consideration for regulatory approval. ${ }^{453,454}$ Initial results from patients with $\mathrm{HF}$ are available and confirm the efficacy of these therapies in reducing serum potassium $^{455}$ and preventing recurrent hyperkalaemia in patients with HF and CKD in the context of treatment with RAAS inhibitors. $^{456}$

\subsection{Hyperlipidaemia}

Elevated low-density lipoprotein cholesterol is uncommon in HFrEF; patients with advanced HFrEF often have low concentrations of low-density lipoprotein, which is associated with a worse prognosis. Rosuvastatin did not reduce the primary composite mortality/ morbidity endpoints in two large RCTs in patients with HF with or without IHD, but it also did not increase risk, and may have reduced, hospitalizations. ${ }^{205,457}$ Therefore there is no evidence to recommend the initiation of statins in most patients with HF. However, in patients who are already receiving a statin for CAD, a continuation of this therapy may be considered.

\subsection{Hypertension}

Hypertension is associated with an increased risk of developing HF; antihypertensive therapy markedly reduces the incidence of $\mathrm{HF}$ (with an exception of $\alpha$-adrenoceptor blockers, which are less effective than other antihypertensives in preventing HF). ${ }^{458}$ A recent prospective cohort study documented that in a population with incident HF, higher baseline systolic, diastolic and pulse pressure levels were associated with a higher rate of adverse events, which further supports the importance for optimized blood pressure control in this population. ${ }^{459}$ Blood pressure control is an element of the holistic management of patients with HF.

Negatively inotropic CCBs (i.e. diltiazem and verapamil) should not be used to treat hypertension in patients with HFrEF (but are believed to be safe in HFpEF), and moxonidine should also be avoided in patients with $\mathrm{HFrEF}$, as it increased mortality in patients in one RCT. ${ }^{460}$ If blood pressure is not controlled with an ACEI (or an ARB), a beta-blocker, an MRA and a diuretic, then hydralazine and amlodipine ${ }^{215}$ [or felodipine ${ }^{216}$ ] are additional blood pressure lowering agents that have been shown to be safe in systolic HF. The blood pressure targets recommended in hypertension guidelines $^{317}$ are applicable to HF. Uncontrolled hypertension in patients with $\mathrm{HFrEF}$ is very rare, provided they are optimally treated for HF. In contrast, treatment of hypertension is an important issue in patients with HFpEF. In patients with AHF, i.v. nitrates (or sodium nitroprusside) are recommended to lower blood pressure (see Section 12). 


\section{Recommendations for the treatment of hypertension in patients with symptomatic (NYHA Class II-IV) heart failure with reduced ejection fraction}

\begin{tabular}{l}
\hline Recommendations \\
\hline Step I \\
ACE-I (or ARB), a beta-blocker or an MRA (or a combination) is recommended to reduce blood pressure as first-, second- and \\
third-line therapy, respectively, because of their associated benefits in HFrEF (reducing the risk of death and HF hospitalization). \\
They are also safe in HFpEF.
\end{tabular}

\begin{tabular}{|c|c|c|}
\hline Class $^{a}$ & Level $^{\mathrm{b}}$ & $\operatorname{Ref}^{c}$ \\
\hline I & A & $\begin{array}{c}2,164, \\
165,167, \\
168, \\
17 \mid-174, \\
182, \\
46 \mid-463\end{array}$ \\
\hline
\end{tabular}

\section{Step 2}

A thiazide diuretic (or if the patient is being treated with a thiazide diuretic, switching to a loop diuretic) is recommended to reduce blood pressure when hypertension persists despite treatment with a combination of an ACE-I (or alternatively ARB but NOT together withan ACE-I), a beta-blocker and an MRA.

\begin{tabular}{|c|c|c|c|}
\hline \multicolumn{4}{|l|}{ Step 3} \\
\hline $\begin{array}{l}\text { Amlodipine or hydralazine is recommended to reduce blood pressure when hypertension persists despite treatment with a } \\
\text { combination of an ACE-I (or alternatively ARB but NOT together withan ACE-I), a beta-blocker, an MRA and a diuretic. }\end{array}$ & I & A & $\begin{array}{l}183,184 \\
215,409\end{array}$ \\
\hline Moxonidine is not recommended to reduce blood pressure because of safety concerns in HFrEF patients (increased mortality). & III & B & 460 \\
\hline $\begin{array}{l}\text { Diltiazem and verapamil are not recommended to reduce blood pressure in patients with HFrEF because of their negative } \\
\text { inotropic action and risk of worsening HF. }\end{array}$ & III & C & 214 \\
\hline
\end{tabular}

$\mathrm{ACE}=$ angiotensin-converting enzyme; $\mathrm{ARB}=$ angiotensin receptor blocker; $\mathrm{HF}=$ heart failure; $\mathrm{HFmrEF}=$ heart failure with mid-range ejection fraction; $\mathrm{HFpEF}=$ heart failure with preserved ejection fraction; HFrEF = heart failure with reduced ejection fraction; MRA = mineralocorticoid receptor antagonist; NYHA = New York Heart Association. ${ }^{a}$ Class of recommendation.

bLevel of evidence.

${ }^{\mathrm{c}}$ Reference(s) supporting recommendations.

\subsection{Iron deficiency and anaemia}

Iron deficiency is common in HF, as it is with other chronic illnesses, and it can lead to anaemia and/or skeletal muscle dysfunction without anaemia. ${ }^{466}$ Within an HF population, iron deficiency is associated with a worse prognosis. ${ }^{467,468}$ Intravenous iron has been specifically studied in two RCTs in patients with HF and iron deficiency (serum ferritin $<100 \mu \mathrm{g} / \mathrm{L}$ or ferritin between 100 and $299 \mu \mathrm{g} / \mathrm{L}$ and transferrin saturation $<20 \%)^{469,470}$ both with and without anaemia. Intravenous ferric carboxymaltose (FCM) has been shown to improve self-reported patient global assessment, quality of life and NYHA class (over 6 months) in the FAIR-HF trial ${ }^{469}$ both in anaemic and non-anaemic patients with $\mathrm{HF}^{471}$ and in the CONFIRM-HF trial $^{470}$, exercise capacity improved over 24 weeks. In the analysis of secondary endpoints in the CONFIRM-HF trial, i.v. iron reduced the risk of HF hospitalizations in iron-deficient patients with HFrEF. ${ }^{470}$ A meta-analysis of i.v. iron therapy in HFrEF patients with iron deficiency over up to 52 weeks showed reduced hospitalization rates and improved HF symptoms, exercise capacity and quality of life. ${ }^{472}$ Treatment with FCM may therefore result in sustainable improvement in functional capacity, symptoms and quality of life. Treatment was also associated with a significant reduction in hospitalizations for worsening HF. The number of deaths and the incidence of adverse events were similar. Neither i.v. iron trial was powered to test for an effect on major outcomes or to evaluate separately the effects in anaemic and non-anaemic patients. The effect of treating iron deficiency in HFpEF/HFmrEF and the long-term safety of iron therapy in either HFrEF, HFmrEF or HFpEF is unknown. The safety of i.v. iron is unknown in patients with $\mathrm{HF}$ and haemoglobin $>15 \mathrm{~g} / \mathrm{dL} .{ }^{469,470}$ Patients with iron deficiency need to be screened for any potentially treatable/reversible causes (e.g. gastrointestinal sources of bleeding). 


\section{Recommendations for the treatment of other co-morbidities in patients with heart failure}

\begin{tabular}{|l|c|c|c|}
\hline Recommendations & Class $^{\mathbf{a}}$ & Level $^{\mathrm{b}}$ & Ref $^{\mathrm{c}}$ \\
\hline Iron deficiency & & & \\
\hline $\begin{array}{l}\text { Intravenous FCM should be } \\
\text { considered in symptomatic patients } \\
\text { with HFrEF and iron deficiency } \\
\text { (serum ferritin }<100 \mu \mathrm{L} / \mathrm{L}, \text { or } \\
\text { ferritin between } 100-299 \mu \mathrm{g} / \mathrm{L} \text { and } \\
\text { transferrin saturation }<20 \%) \text { in } \\
\text { order to alleviate HF symptoms, } \\
\text { and improve exercise capacity and } \\
\text { quality of life. }\end{array}$ & Ila & A & 469,470 \\
\hline Diabetes & & \\
\hline $\begin{array}{l}\text { Metformin should be considered as } \\
\text { a first-line treatment of glycaemic } \\
\text { control in patients with diabetes } \\
\text { and HF, unless contra-indicated. }\end{array}$ & \multicolumn{1}{|l}{} & \\
\hline
\end{tabular}

$\mathrm{FCM}=$ ferric carboxymaltose; $\mathrm{HF}=$ heart failure; $\mathrm{HFrEF}=$ heart failure with reduced ejection fraction.

${ }^{\mathrm{a}}$ Class of recommendation.

'Level of evidence.

'Reference(s) supporting recommendations.

Treatments not recommended for other co-morbidities in patients with heart failure

Treatments not recommended of other co-morbidities in patients with heart failure

\begin{tabular}{|c|c|c|c|}
\hline Recommendations & Class $^{a}$ & Level $^{\mathrm{b}}$ & $\operatorname{Ref}^{c}$ \\
\hline \multicolumn{4}{|l|}{ Sleep apnoea } \\
\hline $\begin{array}{l}\text { Adaptive servo-ventilation is } \\
\text { not recommended in patients } \\
\text { with } \mathrm{HFrEF} \text { and a predominant } \\
\text { central sleep apnoea because } \\
\text { of an increased all-cause and } \\
\text { cardiovascular mortality. }\end{array}$ & III & B & 473 \\
\hline \multicolumn{4}{|l|}{ Diabetes } \\
\hline $\begin{array}{l}\text { Thiazolidinediones (glitazones) are } \\
\text { not recommended in patients with } \\
\mathrm{HF} \text {, as they increase the risk of HF } \\
\text { worsening and HF hospitalization. }\end{array}$ & III & A & 209,210 \\
\hline \multicolumn{4}{|l|}{ Arthritis } \\
\hline $\begin{array}{l}\text { NSAIDs or COX-2 inhibitors are } \\
\text { not recommended in patients with } \\
\mathrm{HF} \text {, as they increase the risk of HF } \\
\text { worsening and HF hospitalization. }\end{array}$ & III & B & $2|I-2| 3$ \\
\hline
\end{tabular}

COX-2 = cyclooxygenase $2 ; \mathrm{HF}=$ heart failure; $\mathrm{HFrEF}=$ heart failure with reduced ejection fraction; NSAID = non-steroidal anti-inflammatory drug.

${ }^{a}$ Class of recommendation.

'Level of evidence.

${ }^{\mathrm{c}}$ Reference(s) supporting recommendations.

Anaemia (defined as a haemoglobin concentration $<13.0 \mathrm{~g} / \mathrm{dL}$ in men and $<12.0 \mathrm{~g} / \mathrm{dL}$ in women) is common in HF, particularly in hospitalized patients. It is more common in women, the elderly and in patients with renal impairment and is associated with advanced myocardial remodelling, inflammation and volume overload. ${ }^{474}$ Anaemia is associated with advanced symptoms, worse functional status, greater risk of HF hospitalization and reduced survival. A diagnostic workup to seek a cause for any finding of anaemia is indicated (e.g. occult blood loss, iron deficiency, B12/folate deficiency, blood dyscrasias), although in many patients no specific cause is found. The erythropoietin-stimulating agent darbepoetin alfa did not improve clinical outcomes in HFrEF patients with mild to moderate anaemia, but led to an excess of thromboembolic events and is therefore not recommended. ${ }^{475}$

\subsection{Kidney dysfunction (including chronic kidney disease, acute kidney injury, cardio-renal syndrome and prostatic obstruction)}

HF and CKD frequently coexist, share many risk factors (diabetes, hypertension, hyperlipidaemia) and interact to worsen prognosis. ${ }^{476,477} \mathrm{CKD}$ is generally defined as an eGFR $<60 \mathrm{~mL} / \mathrm{min} /$ $1.73 \mathrm{~m}^{2}$ and/or the presence of albuminuria (high 30-300 or very high $>300 \mathrm{mg}$ albumin/1 g of urine creatinine). Patients with severe renal dysfunction (eGFR $<30 \mathrm{~mL} / \mathrm{min} / 1.73 \mathrm{~m}^{2}$ ) have systematically been excluded from randomized clinical trials and therefore there is lack of evidence-based therapies in these patients.

A further deterioration in renal function, termed worsening renal function (WRF), is used to indicate an increase in serum creatinine, usually by $>26.5 \mu \mathrm{mol} / \mathrm{L}(0.3 \mathrm{mg} / \mathrm{dL})$ and/or a $25 \%$ increase or a $20 \%$ drop in GFR. The importance of these apparently small changes is that they are frequent, they promote the development and progression of $\mathrm{CKD}^{478}$ and, as a consequence, can worsen the prognosis of HF. Increases in creatinine during an AHF hospitalization are not always clinically relevant, especially when they are accompanied by appropriate decongestion, diuresis and haemoconcentration. ${ }^{479}$

Large increases in serum creatinine, termed acute kidney injury (AKI), are relatively rare in HF and are probably associated with the combination of diuretic therapy with other potentially nephrotoxic drugs such as some antibiotics (gentamicin and trimethoprim), contrast media, ACEls, ARBs, NSAIDs, etc. Of relevance, some of these drugs may accumulate if they are renally excreted. In HF, WRF is relatively common, especially during initiation and uptitration of RAAS inhibitor therapy. Despite the fact that RAAS blockers can frequently cause a decrease in GFR in patients with $\mathrm{HF}$, this reduction is usually small and should not lead to treatment discontinuation unless there is a marked decrease, as the treatment benefit in these patients is probably largely maintained. ${ }^{480}$ When large increases in serum creatinine occur, care should be taken to evaluate the patient thoroughly and should include assessment of a possible renal artery stenosis, excessive hyper- or hypovolaemia, concomitant medication and hyperkalaemia, which frequently coincides with WRF.

Diuretics, especially thiazides, but also loop diuretics, may be less effective in patients with a very low GFR, and if used, should be dosed appropriately (higher doses to achieve similar effects). Renally excreted drugs (e.g. digoxin, insulin and low molecular weight heparin) may accumulate in patients with renal impairment and may need dose adjustment if renal function deteriorates. Patients with HF and coronary or peripheral vascular disease are at risk of acute renal dysfunction when they undergo contrast media enhanced angiography [contrast-induced acute 
kidney injury (Cl-AKI)]. Renal dysfunction and worsening renal function is further discussed in the section about AHF (see Section 12).

Prostatic obstruction is common in older men and can interfere with renal function; it should therefore be ruled out in men with HF with deteriorating renal function. $\alpha$-adrenoceptor blockers cause hypotension and sodium and water retention, and may not be safe in HFrEF. ${ }^{458,464,465}$ For these reasons, 5 - $\alpha$-reductase inhibitors are generally preferred in the medical treatment of prostatic obstruction in patients with HF.

\subsection{Lung disease (including asthma and chronic obstructive pulmonary disease)}

The diagnosis of COPD and asthma may be difficult in patients with $\mathrm{HF}$, due to overlap in symptoms and signs, but also problems in the interpretation of spirometry, especially in HFpEF. ${ }^{48,49,391}$ COPD (and asthma) in patients with HF may be overdiagnosed. ${ }^{481}$ Spirometry should be performed when patients have been stable and euvolaemic for at least 3 months, to avoid the confounding effect of pulmonary congestion causing external obstruction of alveoli and bronchioles. ${ }^{482}$ Both correctly and incorrectly labelled COPD are associated with worse functional status and a worse prognosis in HFrEF.

Beta-blockers are only relatively contraindicated in asthma, but not in COPD, although a more selective $\beta 1$-adrenoceptor antagonist (i.e. bisoprolol, metoprolol succinate, or nebivolol) is preferred. ${ }^{48,49,391}$ The contraindication to beta-blockers in asthma, as mentioned on pharmacy leaflets, is based on small case series published in the 1980s and late 1990s with very high initial dosages in young patients with severe asthma. In clinical practice, starting with low doses of cardioselective beta-blockers combined with close monitoring for signs of airway obstruction (wheezing, shortness of breath with lengthening of the expiration) may allow the use of profoundly effective beta-blockers in HFrEF, especially in older people where true severe asthma is uncommon. Therefore, according to the 2015 GINA global strategy report, ${ }^{395,396}$ asthma is not an absolute contraindication, but these medications should only be used under close medical supervision by a specialist, with consideration of the risks for and against their use. The long-term safety of cardioactive inhaled pulmonary drugs is uncertain and the need for their use should be reconsidered in patients with HFrEF, especially as their benefit in asthma and COPD may be symptomatic only without a clear effect on mortality. Oral corticosteroids can cause sodium and water retention, potentially leading to worsening of HF, but this is not believed to be a problem with inhaled corticosteroids. Pulmonary hypertension can complicate severe long-standing COPD, which, as a result, makes right-sided HF and congestion more likely. Non-invasive ventilation, added to conventional therapy, improves the outcome of patients with acute respiratory failure due to hypercapnic exacerbation of COPD or HF in situations of acute pulmonary oedema.

\subsection{Obesity}

Obesity is a risk factor for $\mathrm{HF}^{141}$ and complicates its diagnosis, because it can cause dyspnoea, exercise intolerance and ankle swelling and may result in poor-quality echocardiographic images. Obese individuals also have reduced NP levels. ${ }^{62}$ Obesity is more common in HFpEF than in $\mathrm{HFrEF}$, although it is possible that misdiagnosis may explain at least some of this difference in prevalence. Although obesity is an independent risk factor for developing HF, once HF is diagnosed, it is well established that obesity is associated with lower mortality across a wide range of body mass indexes (BMls) (see also cachexia in Section 11.3) — the so-called obesity paradox also seen in other chronic illnesses. ${ }^{414,416}$ Obesity should be managed as recommended in the ESC guidelines on cardiovascular disease prevention, ${ }^{483}$ if the aim is to prevent future development of HF. However, these guidelines do not refer to the HF patient in whom higher BMI is not adverse, and, although often recommended for symptom benefit and risk factor control, weight loss as an intervention has never been prospectively shown to be either beneficial or safe in HFrEF. When weight loss is occurring in HF, it is associated with high mortality and morbidity, worse symptom status and poor quality of life. In patients with HF with moderate degrees of obesity (BMI $<35 \mathrm{~kg} / \mathrm{m}^{2}$ ), weight loss cannot be recommended. In more advanced obesity (BMI $35-45 \mathrm{~kg} / \mathrm{m}^{2}$ ), weight loss may be considered to manage symptoms and exercise capacity.

\subsection{Sleep disturbance and sleep-disordered breathing}

Sleep-disordered breathing (SDB) occurs in more than one-third of patients with $\mathrm{HF}^{484}$ being even more prevalent in patients with AHF. ${ }^{485}$ The most common types are: central sleep apnoea (CSA, similar to Cheyne Stokes respiration, CSR), obstructive sleep apnoea (OSA), and a mixed pattern of the two. Other causes of sleep disturbance include anxiety, depression, decubitus or paroxysmal pulmonary congestion (orthopnoea and paroxysmal nocturnal dyspnoea) and diuretic therapy causing nocturnal diuresis. Reviewing sleep history (including asking a partner) is part of the holistic care of patients with HF (see Section 14). CSA and OSA have been shown to be associated with a worse prognosis in HF. ${ }^{485,486}$ OSA is associated with an increased risk of incident HF in men. ${ }^{487}$ CSA is the most common form of SDB in HFrEF, and HFrEF is the most common cause of CSA, so they are closely linked. Screening for, and the diagnosis and treatment of, sleep apnoea is discussed in detail elsewhere. ${ }^{484,488}$ Diagnosis used to require overnight polysomnography, although advanced home testing equipment which can distinguish the type of sleep apnoea has been developed.

Nocturnal oxygen supplementation, continuous positive airway pressure (CPAP), bi-level positive airway pressure (BiPAP), and adaptive servo-ventilation (ASV) may be considered to treat nocturnal hypoxaemia in OSA as recommended in other guidelines. ${ }^{489,490} \mathrm{An}$ apnoea/hypopnoea index (AHI) of above 30 per hour can be treated using any of CPAP, BiPAP, ASV and nocturnal oxygen supplementation, which have all been shown to be effective in this regard. It should be noted, however, that none of these interventions has been prospectively shown to be beneficial on major outcomes in HFrEF.

CPAP in HF related CSA has been shown to reduce the frequency of episodes of apnoea and hypopnoea, and improve LVEF and 6 minute walk test distance, but did not improve prognosis or the rate of $\mathrm{HF}$ related hospitalizations. ${ }^{491}$

The recently published SERVE-HF ${ }^{473}$ trial has shown that ASV used in patients with HFrEF and a predominantly CSA was neutral regarding the composite primary endpoint (all-cause death, lifesaving cardiovascular intervention, i.e. cardiac transplantation, implantation of a ventricular assist device, resuscitation after sudden cardiac arrest, or appropriate lifesaving shock, or unplanned hospitalization for HF worsening), but more importantly led to an increase in both all-cause and cardiovascular mortality. Therefore ASV is not recommended in patients with HFrEF and predominantly CSA. 
The safety and efficacy of alternative approaches to treating CSA in HFrEF patients, such as implantable phrenic nerve stimulation, ${ }^{219,220,492}$ are presently undergoing clinical investigation and may require additional long term study.

\subsection{Valvular heart disease}

Valvular heart disease may cause or aggravate HF. This section briefly addresses problems particularly relevant to $\mathrm{HF}$, and the reader is referred to the recent guidelines on valvular disease for more information. ${ }^{493,494}$

Patients with HFand concomitant valvular heart disease constitute a high-risk population. Thus, the whole process of decision-making through a comprehensive evaluation of the risk-benefit ratio of different treatment strategies should be made by a multidisciplinary "heart team' with a particular expertise in valvular heart disease, including cardiologists with expertise in $\mathrm{HF}$, cardiac surgeons, a structural valve interventionist if a catheter-based therapy is being considered, imaging specialists, anaesthetists and, if needed, general practitioners, geriatricians, or intensive care specialists. This may be particularly beneficial in patients with HF being considered for surgery, transcatheter aortic valve implantation or transcatheter mitral valve intervention.

All patients should receive OMT. In those with HFrEF pharmacological therapy should be planned according to a previously described algorithm (see Section 7 for details). Care must be taken using vasodilators (ACEI, ARBs, CCBs, hydralazine, and nitrates) in patients with severe aortic stenosis in order not to cause hypotension.

\subsubsection{Aortic stenosis}

The main concern in patients with severe aortic stenosis and reduced LVEF is the entity of 'low-flow, low-gradient' aortic stenosis (valve area $<1 \mathrm{~cm}^{2}$, LVEF $<40 \%$, mean pressure gradient $<40$ $\mathrm{mmHg}$ ). In such individuals, low-dose dobutamine stress echocardiography should be considered to differentiate between patients with moderate aortic stenosis, and those with severe stenosis and low flow across the valve due to low stroke volume, and to evaluate for contractile or flow reserve.

If the mean gradient is $>40 \mathrm{mmHg}$, there is theoretically no lower LVEF limit for aortic valve replacement in symptomatic patients with severe aortic stenosis.

Transaortic valve implantation (TAVI) is recommended in patients with severe aortic stenosis who are not suitable for surgery as assessed by a 'heart team' and have predicted post-TAVI survival $>1$ year. TAVI should be also considered in high-risk patients with severe aortic stenosis who may still be suitable for surgery, but in whom TAVI is favoured by a 'heart team' based on the individual risk profile and anatomic suitability. ${ }^{495,496}$ In a recent trial in patients with severe aortic stenosis, TAVI with a self-expanding transcatheter aortic valve bioprosthesis was associated with a significantly higher rate of survival at 1 year which was sustained at 2 years. ${ }^{497,498}$

\subsubsection{Aortic regurgitation}

In patients with severe aortic regurgitation, aortic valve repair or replacement is recommended in all symptomatic patients and in asymptomatic patients with resting LVEF $\leq 50 \%$, who are otherwise fit for surgery. ${ }^{499,500}$

\subsubsection{Mitral regurgitation}

This section refers to chronic settings while acute settings are discussed in Section 12.

\section{Primary (organic) mitral regurgitation}

Surgery is indicated in symptomatic patients with severe organic mitral regurgitation with no contra-indications to surgery. The decision of whether to replace or repair depends mostly on valve anatomy, surgical expertise available, and the patient's condition.

When the LVEF is $<30 \%$, a durable surgical repair may improve symptoms, although its effect on survival is unknown. In this situation, the decision to operate should take account of response to medical therapy, co-morbidities, and the likelihood that the valve can be repaired (rather than replaced).

\section{Secondary mitral regurgitation}

This occurs because LV enlargement and remodelling lead to reduced leaflet closing. Effective medical therapy (including CRT in suitable patients) leading to reverse remodelling of the LV may reduce functional mitral regurgitation, and every effort should be made to optimize medical treatment in these patients.

Combined valve and coronary surgery should be considered in symptomatic patients with LV systolic dysfunction (LVEF < 30\%), coronary arteries suitable for revascularization, and evidence of viability. Surgery is also recommended in patients with severe mitral regurgitation undergoing CABG with LVEF $>30 \%$.

However, a recent study in patients with moderate, secondary ischaemic mitral regurgitation did not prove that the addition of mitral valve repair to CABG would lead to a higher degree of $L V$ reverse remodelling. ${ }^{501}$ Also, there is no evidence favouring mitral valve repair over replacement in the context of better outcomes and magnitude of $L V$ remodelling. ${ }^{502}$ In the presence of $A F$, atrial ablation and LA appendage closure may be considered at the time of mitral valve surgery.

The role of isolated mitral valve surgery in patients with severe functional mitral regurgitation and severe LV systolic dysfunction (LVEF < 30\%) who cannot be revascularized or have non-ischaemic cardiomyopathy is questionable, and in most patients conventional medical and device therapy are preferred. In selected cases, repair may be considered in order to avoid or postpone transplantation. The decision should be based on comprehensive evaluation (including strain echocardiography or magnetic resonance imaging ${ }^{499,503}$ and discussed within the 'heart team'.

In patients with HF with moderate-severe, secondary mitral regurgitation who are judged inoperable or at high surgical risk, percutaneous mitral valve intervention (percutaneous edge-to-edge repair) may be considered in order to improve symptoms and quality of life, although no RCT evidence of improvement has been published, only registry studies. ${ }^{504-506}$

\subsubsection{Tricuspid regurgitation}

Secondary (functional) tricuspid regurgitation (TR) frequently complicates the natural course of HF, due to annular dilatation and increased tricuspid leaflet tethering in relation to RV pressure and/ or volume overload. Severe TR causes/deteriorates symptoms and signs of right HF, thus diuretics are used to reduce peripheral oedema. As hepatic congestion is often present in these patients (additionally contributing to hyperaldosteronism), an addition of an MRA (in higher natriuretic doses) may improve decongestion. ${ }^{507}$ Management of HF which underlies secondary TR should be optimized as TR may diminish, following the treatment of its cause. Indications for surgical correction of secondary TR complicating HF are not clearly established. ${ }^{493,494}$ The need for correction of TR is usually considered at the time of surgical correction of left-sided 


\section{Recommendations for treatment of valvular diseases in patients with heart failure}

\begin{tabular}{|c|c|c|c|}
\hline Recommendations & Class $^{a}$ & Level $^{\mathrm{b}}$ & $\operatorname{Ref}^{c}$ \\
\hline $\begin{array}{l}\text { In symptomatic patients with reduced LVEF and 'low-flow, low-gradient' aortic stenosis (valve area }<1 \mathrm{~cm}^{2} \text {, LVEF }<40 \% \text {, mean } \\
\text { pressure gradient }<40 \mathrm{mmHg} \text { ), low-dose dobutamine stress echocardiography should be considered to identify those with severe } \\
\text { aortic stenosis suitable for valve replacement. }\end{array}$ & Ila & c & \\
\hline $\begin{array}{l}\text { TAVI is recommended in patients with severe aortic stenosis who are not suitable for surgery as assessed by a 'heart team' } \\
\text { and have predicted post-TAVI survival }>\text { I year. }\end{array}$ & $\mathbf{I}$ & B & $\begin{array}{l}495,496, \\
509\end{array}$ \\
\hline $\begin{array}{l}\text { TAVI should be considered in high-risk patients with severe aortic stenosis who may still be suitable for surgery, but in whom TAVI is } \\
\text { favoured by a 'heart team' based on the individual risk profile and anatomic suitability. }\end{array}$ & Ila & A & 497,498 \\
\hline $\begin{array}{l}\text { In patients with severe aortic regurgitation, aortic valve repair or replacement is recommended in all symptomatic patients and } \\
\text { in asymptomatic patients with resting LVEF } \leq 50 \% \text {, who are otherwise fit for surgery. }\end{array}$ & $\mathbf{I}$ & C & 317 \\
\hline Evidence-based medical therapy in patients with HFrEF is recommended in order to reduce functional mitral regurgitation. & $\mathbf{I}$ & C & \\
\hline $\begin{array}{l}\text { Combined surgery of secondary mitral regurgitation and coronary artery bypass grafting should be considered in symptomatic } \\
\text { patients with } \mathrm{LV} \text { systolic dysfunction (LVEF }<30 \%) \text {, requiring coronary revascularization for angina recalcitrant to medical therapy. }\end{array}$ & Ila & C & \\
\hline $\begin{array}{l}\text { Isolated surgery of non-ischaemic regurgitant mitral valve in patients with severe functional mitral regurgitation and severe LV systolic } \\
\text { dysfunction (LVEF }<30 \%) \text { may be considered in selected patients in order to avoid or postpone transplantation. }\end{array}$ & Illb & C & \\
\hline
\end{tabular}

$\mathrm{HFrEF}=$ heart failure with reduced ejection fraction; $\mathrm{LV}=$ left ventricular; $\mathrm{LVEF}=$ left ventricular ejection fraction; TAVI = transaortic valve implantation.

${ }^{a}$ Class of recommendation.

bevel of evidence.

'Reference(s) supporting recommendations.

valve lesions. ${ }^{493,494} \mathrm{~A}$ recent first report indicated that catheterbased interventions may be possible for TR. ${ }^{508}$

\section{Acute heart failure}

\subsection{Definition and classification}

AHF refers to rapid onset or worsening of symptoms and/or signs of HF. It is a life-threatening medical condition requiring urgent evaluation and treatment, typically leading to urgent hospital admission.

AHF may present as a first occurrence (de novo) or, more frequently, as a consequence of acute decompensation of chronic $\mathrm{HF}$, and may be caused by primary cardiac dysfunction or precipitated by extrinsic factors, often in patients with chronic HF. Acute myocardial dysfunction (ischaemic, inflammatory or toxic), acute valve insufficiency or pericardial tamponade are among the most frequent acute primary cardiac causes of AHF. Decompensation of chronic HF can occur without known precipitant factors, but more often with one or more factors, such as infection, uncontrolled hypertension, rhythm disturbances or non-adherence with drugs/diet (Table 12.1).

A large number of overlapping classifications of AHF based on different criteria have been proposed. ${ }^{510-513}$ In practice the most useful classifications are those based on clinical presentation at admission, allowing clinicians to identify patients at high risk of complications and to direct management at specific targets, which creates a pathway for personalized care in the AHF setting. In most cases, patients with AHF present with either preserved (90-140 $\mathrm{mmHg}$ ) or elevated (>140 $\mathrm{mmHg}$; hypertensive AHF) systolic blood pressure (SBP). Only 5-8\% of all patients present with low SBP (i.e. $<90 \mathrm{mmHg}$; hypotensive AHF), which is associated with poor prognosis, particularly when hypoperfusion is also present. $^{514,515}$

\section{Table I 2. I Factors triggering acute heart failure}

\begin{tabular}{|l|}
\hline Acute coronary syndrome. \\
\hline Tachyarrhythmia (e.g. atrial fibrillation, ventricular tachycardia). \\
\hline Excessive rise in blood pressure. \\
\hline Infection (e.g. pneumonia, infective endocarditis, sepsis). \\
\hline Non-adherence with salt/fluid intake or medications. \\
\hline Bradyarrhythmia. \\
\hline Toxic substances (alcohol, recreational drugs). \\
\hline $\begin{array}{l}\text { Drugs (e.g. NSAIDs, corticosteroids, negative inotropic substances, } \\
\text { cardiotoxic chemotherapeutics). }\end{array}$ \\
\hline Exacerbation of chronic obstructive pulmonary disease. \\
\hline Pulmonary embolism. \\
\hline Surgery and perioperative complications. \\
\hline Increased sympathetic drive, stress-related cardiomyopathy. \\
\hline $\begin{array}{l}\text { Metabolic/hormonal derangements (e.g. thyroid dysfunction, diabetic } \\
\text { ketosis, adrenal dysfunction, pregnancy and peripartum related } \\
\text { abnormalities). }\end{array}$ \\
\hline Cerebrovascular insult. \\
\hline $\begin{array}{l}\text { Acute mechanical cause: myocardial rupture complicating ACS (free wall } \\
\text { rupture,ventricular septal defect,acute mitral regurgitation), chest trauma } \\
\text { or cardiac intervention, acute native or prosthetic valve incompetence } \\
\text { secondary to endocarditis, aortic dissection or thrombosis. }\end{array}$ \\
\hline
\end{tabular}

ACS $=$ acute coronary syndromes; NSAIDs $=$ non-steroidal anti-inflammatory drugs.

Another approach is to classify patients according to the presence of the following precipitants/causes leading to decompensation, which need to be treated/corrected urgently (see Section 12.3.1): ACS, hypertensive emergency, rapid arrhythmias or severe 


\section{CONGESTION (-)}

\author{
CONGESTION (+) \\ Pulmonary congestion \\ Orthopnoea/paroxysmal nocturnal dyspnoea \\ Peripheral (bilateral) oedema \\ Jugular venous dilatation \\ Congested hepatomegaly \\ Gut congestion, ascites \\ Hepatojugular reflux
}

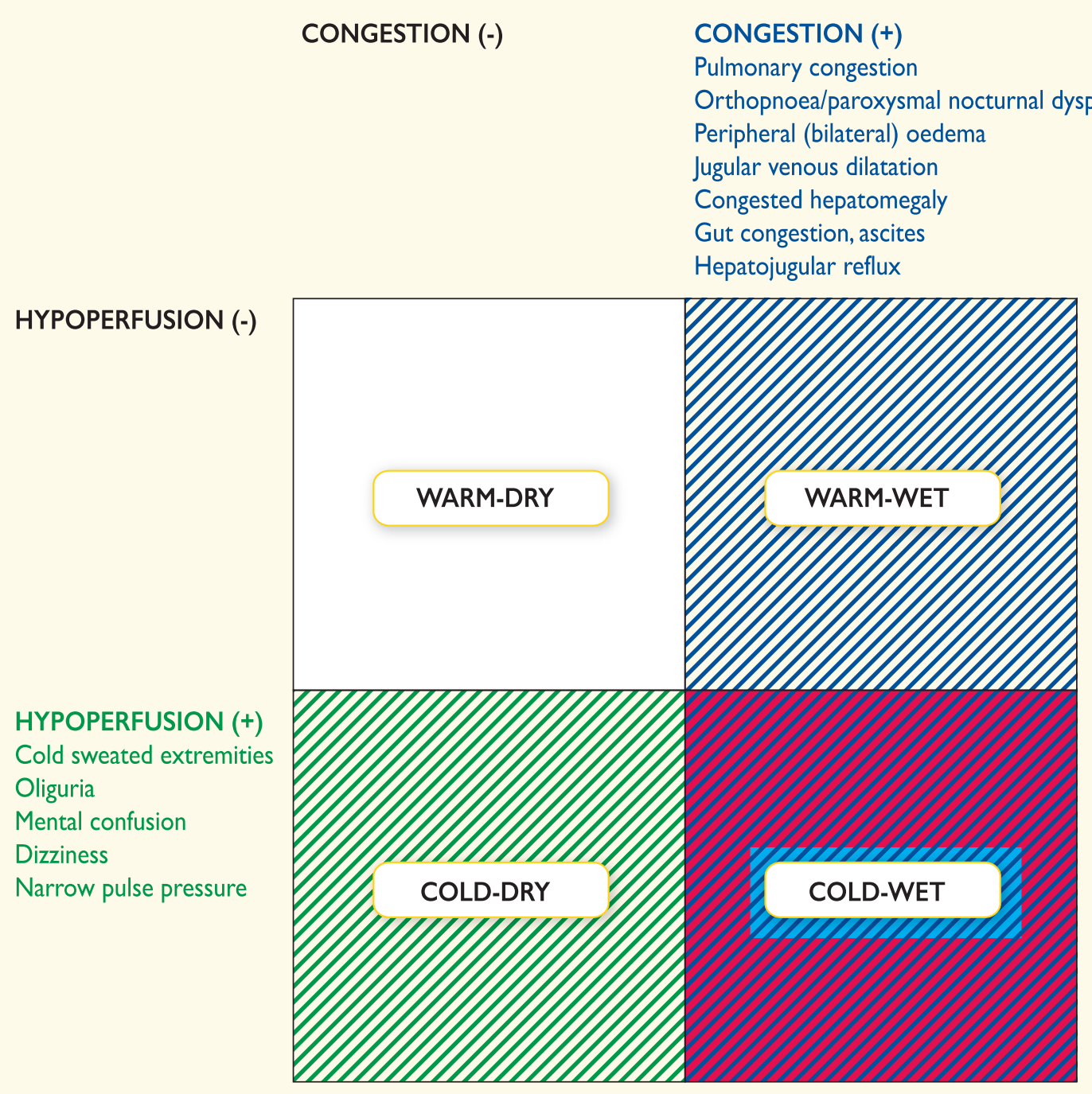

Hypoperfusion is not synonymous with hypotension, but often hypoperfusion is accompanied by hypotension. bradycardia/conduction disturbance, acute mechanical cause underlying AHF or acute pulmonary embolism.

Clinical classification can be based on bedside physical examination in order to detect the presence of clinical symptoms/signs of congestion ('wet' vs. 'dry' if present vs. absent) and/or peripheral hypoperfusion ('cold' vs. 'warm' if present vs. absent) (Figure 12.1). ${ }^{514,515}$ The combination of these options identifies four groups: warm and wet (well perfused and congested) —most commonly present; cold and wet (hypoperfused and congested); cold and dry (hypoperfused without congestion); and warm and dry (compensated, well perfused without congestion). This classification may be helpful to guide therapy in the initial phase and carries prognostic information. ${ }^{510,514,515}$

Patients with HF complicating AMI can be classified according to Killip and Kimball ${ }^{13}$ into class I, no clinical signs of HF; class II, HF with rales and $\mathrm{S}_{3}$ gallop; class III, with frank acute pulmonary oedema; class IV, cardiogenic shock, hypotension (SBP $<90 \mathrm{mmHg}$ ) and evidence of peripheral vasoconstriction such as oliguria, cyanosis and diaphoresis.

Definitions of the terms used in this section related to clinical presentation of patients with AHF are provided in Table 12.2.

\subsection{Diagnosis and initial prognostic evaluation}

The diagnostic workup needs to be started in the pre-hospital setting and continued in the emergency department (ED) in order to establish the diagnosis in a timely manner and initiate appropriate management. The greater benefit of early treatment is well established in ACS and now needs to be considered in the setting of 


\section{Table 1 2.2 Definitions of the terms used in Section 12 on acute heart failure}

\begin{tabular}{|c|c|}
\hline Term & Definition \\
\hline Symptoms/signs of congestion (left-sided) & Orthopnoea, paroxysmal nocturnal dyspnoea, pulmonary rales (bilateral), peripheral oedema (bilateral). \\
\hline Symptoms/signs of congestion (right-sided) & $\begin{array}{l}\text { Jugular venous dilatation, peripheral oedema (bilateral), congested hepatomegaly, hepatojugular reflux, ascites, } \\
\text { symptoms of gut congestion. }\end{array}$ \\
\hline Symptoms/signs of hypoperfusion & $\begin{array}{l}\text { Clinical: cold sweated extremities, oliguria, mental confusion, dizziness, narrow pulse pressure. } \\
\text { Laboratory measures: metabolic acidosis, elevated serum lactate, elevated serum creatinine. } \\
\text { Hypoperfusion is not synonymous with hypotension, but often hypoperfusion is accompanied by hypotension. }\end{array}$ \\
\hline Hypotension & Systolic BP $<90 \mathrm{mmHg}$ \\
\hline Bradycardia & Heart rate $<40 \mathrm{bpm}$ \\
\hline Tachycardia & Heart rate $>120 \mathrm{bpm}$ \\
\hline Abnormal respiratory effort & $\begin{array}{l}\text { Respiratory rate }>25 \text { breaths } / \mathrm{min} \text { with use of accessory muscles for breathing, or respiratory rate } \\
<8 \text { breaths/min despite dyspnoea. }\end{array}$ \\
\hline Low $\mathrm{O}_{2}$ saturation & $\begin{array}{l}\mathrm{O}_{2} \text { saturation }\left(\mathrm{SaO}_{2}\right)<90 \% \text { in pulse oximetry } \\
\text { Normal } \mathrm{SaO}_{2} \text { neither excludes hypoxaemia (low } \mathrm{PaO}_{2} \text { ) nor tissue hypoxia. }\end{array}$ \\
\hline Hypoxaemia & $\mathrm{O}_{2}$ partial pressure $\left(\mathrm{PaO}_{2}\right)$ in arterial blood $<80 \mathrm{mmHg}(<10,67 \mathrm{kPa})$ (blood gas analysis). \\
\hline Hypoxaemic respiratory failure (type I) & $\mathrm{PaO}_{2}<60 \mathrm{mmHg}(<8 \mathrm{kPa})$ \\
\hline Hypercapnia & $\mathrm{CO}_{2}$ partial pressure $\left(\mathrm{PaCO}_{2}\right)$ in arterial blood $>45 \mathrm{mmHg}(>6 \mathrm{kPa})$ (blood gas analysis). \\
\hline Hypercapnic respiratory failure (type II) & $\mathrm{PaCO}_{2}>50 \mathrm{mmHg}(>6,65 \mathrm{kPa})$ \\
\hline Acidosis & $\mathrm{pH}<7.35$ \\
\hline Elevated blood lactate & $>2 \mathrm{mmol} / \mathrm{L}$ \\
\hline Oliguria & Urine output $<0.5 \mathrm{~mL} / \mathrm{kg} / \mathrm{h}$ \\
\hline
\end{tabular}

$\mathrm{BP}=$ blood pressure; $\mathrm{bpm}=$ beats per minute; $\mathrm{PaCO}_{2}=$ partial pressure of carbon dioxide in arterial blood; $\mathrm{PaO}_{2}=$ partial pressure of oxygen in arterial blood; $\mathrm{SaO}=$ oxygen saturation.

AHF. ${ }^{516,517}$ In parallel, coexisting life-threatening clinical conditions and/or precipitants that require urgent treatment/correction need to be immediately identified and managed (Figure 12.2). Typically, an initial step in the diagnostic workup of AHF is to rule out alternative causes for the patient's symptoms and signs (i.e. pulmonary infection, severe anaemia, acute renal failure).

When AHF is confirmed clinical evaluation is mandatory to select further management.

It is recommended that initial diagnosis of AHF should be based on a thorough history assessing symptoms, prior cardiovascular history and potential cardiac and non-cardiac precipitants, as well as on the assessment of signs/symptoms of congestion and/or hypoperfusion by physical examination and further confirmed by appropriate additional investigations such as ECG, chest X-ray, laboratory assessment (with specific biomarkers) and echocardiography.

In patients presenting with AHF, early initiation of appropriate therapy (along with relevant investigations) is of key importance. $^{516-518}$

Typically, symptoms and signs of AHF reflect fluid overload (pulmonary congestion and/or peripheral oedema) or, less often, reduced cardiac output with peripheral hypoperfusion (Table 12.2). Since the sensitivity and specificity of symptoms and signs are often not satisfactory, careful clinical evaluation needs to be followed by these additional investigations:
- Chest X-ray can be a useful test for the diagnosis of AHF. Pulmonary venous congestion, pleural effusion, interstitial or alveolar oedema and cardiomegaly are the most specific findings for AHF, although in up to $20 \%$ of patients with AHF, chest X-ray is nearly normal. ${ }^{519}$ Supine chest radiographs are of limited value in AHF. Chest $X$-ray is also useful to identify alternative non-cardiac diseases that may cause or contribute to the patient's symptoms (i.e. pneumonia, non-consolidative pulmonary infections).

- ECG is rarely normal in AHF (high negative predictive value). ${ }^{520} \mathrm{It}$ is also helpful in identifying underlying cardiac disease and potential precipitants (rapid AF, acute myocardial ischaemia).

- Immediate echocardiography is mandatory only in patients with haemodynamic instability (particularly in cardiogenic shock) and in patients suspected of acute life-threatening structural or functional cardiac abnormalities (mechanical complications, acute valvular regurgitation, aortic dissection). Early echocardiography should be considered in all patients with de novo AHF and in those with unknown cardiac function; however, the optimal timing is unknown (preferably within $48 \mathrm{~h}$ from admission, if the expertise is available). Pocket-size echocardiography may be used as an extension of the clinical examination in the first instance where available. Repeated echocardiography is usually not needed unless there is relevant deterioration in clinical status. Bedside thoracic ultrasound for signs of interstitial oedema and 


\section{Patient with suspected AHF}

\section{Urgent phase}

after first medical

contact
Immediate phase (initial 60-120 minutes)
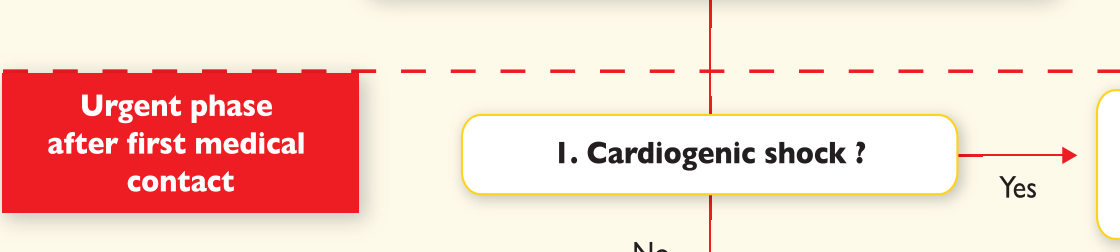

Circulatory support

- pharmacological

- mechanical

No

2. Respiratory failure?

Yes

Ventilatory support

- oxygen

- non-invasive positive pressure ventilation (CPAP, BiPAP)

- mechanical ventilation

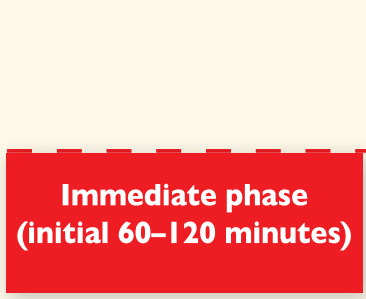

Identification of acute aetiology:

C acute Coronary syndrome

H Hypertension emergency

A Arrhythmia

M acute Mechanical cause ${ }^{a}$

P Pulmonary embolism

Immediate stabilization and transfer to ICU/CCU

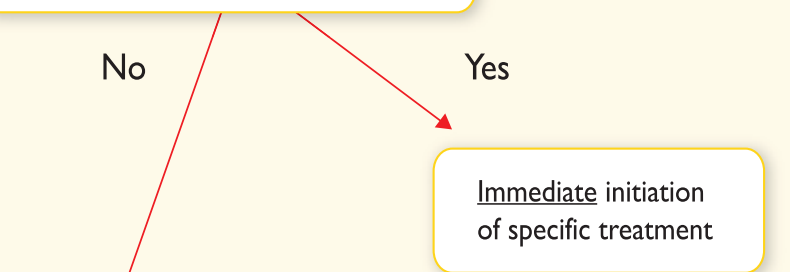

Diagnostic work-up to confirm AHF Clinical evaluation to select optimal management

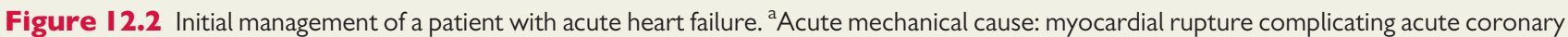
syndrome (free wall rupture, ventricular septal defect, acute mitral regurgitation), chest trauma or cardiac intervention, acute native or prosthetic valve incompetence secondary to endocarditis, aortic dissection or thrombosis, see above. 


\section{Table I 2.3 Causes of elevated concentrations of natriuretic peptides $522-524$}

\begin{tabular}{|l|l|}
\hline Cardiac & Heart failure \\
& Acute coronary syndromes \\
& Pulmonary embolism \\
& Myocarditis \\
& Left ventricular hypertrophy \\
& Hypertrophic or restrictive cardiomyopathy \\
& Valvular heart disease \\
& Congenital heart disease \\
& Atrial and ventricular tachyarrhythmias \\
& Heart contusion \\
& Cardioversion, ICD shock \\
& Surgical procedures involving the heart \\
& Pulmonary hypertension \\
\hline Non-cardiac & Advanced age \\
& Ischaemic stroke \\
& Subarachnoid haemorrhage \\
& Renal dysfunction \\
& Liver dysfunction (mainly liver cirrhosis with ascites) \\
& Paraneoplastic syndrome \\
& Chronic obstructive pulmonary disease \\
& Severe infections (including pneumonia and sepsis) \\
& Severe burns \\
& Anaemia \\
& Severe metabolic and hormone abnormalities \\
& (e.g. thyrotoxicosis, diabetic ketosis) \\
\hline
\end{tabular}

$\mathrm{HFpEF}=$ heart failure with preserved ejection fraction; $\mathrm{HFrEF}=$ heart failure with reduced ejection fraction; ICD = implantable cardioverter defibrillator.

pleural effusion may be useful in detecting AHF if the expertise is available.

- Laboratory tests:

- Natriuretic peptides.

- Upon presentation to the ED or CCU/ICU, a plasma NP level (BNP, NT-proBNP or MR-proANP) should be measured in all patients with acute dyspnoea and suspected AHF to help in the differentiation of AHF from non-cardiac causes of acute dyspnoea. NPs have high sensitivity, and normal levels in patients with suspected AHF makes the diagnosis unlikely (thresholds: BNP $<100 \mathrm{pg} / \mathrm{mL}$, NT-proBNP $<300 \mathrm{pg} / \mathrm{mL}$, MR-proANP $<120 \mathrm{pg} / \mathrm{mL}) .{ }^{57-61,77,78,521}$ However, elevated levels of NPs do not automatically confirm the diagnosis of $\mathrm{AHF}$, as they may also be associated with a wide variety of cardiac and non-cardiac causes (Table 12.3). Unexpectedly low levels of NPs can be detected in some patients with decompensated end-stage HF, flash pulmonary oedema or right sided AHF.

Other laboratory tests at presentation.

- The following laboratory assessments should be performed at admission on the blood of all patients with AHF: cardiac troponin, blood urea nitrogen (BUN) (or urea), creatinine, electrolytes (sodium, potassium), liver function tests, thyroid- stimulating hormone (TSH), glucose and complete blood count; D-dimer is indicated in patients with a suspicion of acute pulmonary embolism.

- Routine arterial blood gas is not needed and should be restricted to patients in whom oxygenation cannot be readily assessed by pulse oximetry. However, arterial blood gas may be useful when a precise measurement of $\mathrm{O}_{2}$ and $\mathrm{CO}_{2}$ partial pressures is needed. A venous sample might acceptably indicate $\mathrm{pH}$ and $\mathrm{CO}_{2}$.

- Of note, measurement of cardiac troponins is useful for detection of ACS as the underlying cause of AHF. However, elevated concentrations of circulating cardiac troponins are detected in the vast majority of patients with AHF, often without obvious myocardial ischaemia or an acute coronary event, suggesting ongoing myocyte injury or necrosis in these patients. ${ }^{525}$ Also in patients with acute pulmonary embolism as the underlying cause of acute decompensation, elevated troponins are useful for risk stratification and decision-making. ${ }^{526}$

- It is recommended to measure creatinine, BUN and electrolytes every 1-2 days while in the hospital and before discharge from the hospital. Of note, more frequent testing might be justified according to the severity of the case. Predischarge assessment of NPs may be considered for prognostic evaluation.

- Assessment of procalcitonin levels may be considered in patients with AHF with suspected coexisting infection, particularly for the differential diagnosis of pneumonia and to guide antibiotic therapy, ${ }^{527}$ if considered.

- Liver function tests are often impaired in patients with AHF due to haemodynamic derangements (both reduced output and increased venous congestion). Abnormal liver function tests identify patients at risk of poor prognosis and may be useful for optimal management. ${ }^{528-530}$

- Since both hypothyroidism and hyperthyroidism may precipitate AHF, TSH should be assessed in newly diagnosed AHF.

- Multiple other biomarkers, including those reflecting inflammation, oxidative stress, neurohormonal disarray and myocardial and matrix remodelling, have been investigated for their diagnostic and prognostic value in AHF; however, none has reached the stage of being recommended for routine clinical use.

- Routine invasive haemodynamic evaluation with a pulmonary artery catheter is not indicated for the diagnosis of AHF. It may be helpful in selected cases of haemodynamically unstable patients with an unknown mechanism of deterioration. Also, routine use of an arterial line or central venous line for diagnostic purposes is not indicated.

Numerous clinical and laboratory variables are independent predictors of in-hospital complications and longer-term outcomes in AHF syndromes, but their impact on management has not been adequately established. 


\section{Recommendations regarding applied diagnostic measurements}

\begin{tabular}{|c|c|c|c|}
\hline Recommendations & Class $^{a}$ & Level $^{\mathrm{b}}$ & Ref $^{c}$ \\
\hline $\begin{array}{l}\text { Upon presentation a measurement of plasma natriuretic peptide level (BNP, NT-proBNP or MR-proANP) is recommended in all } \\
\text { patients with acute dyspnoea and suspected AHF to help in the differentiation of AHF from non-cardiac causes of acute dyspnoea. }\end{array}$ & $\mathbf{I}$ & A & $531-534$ \\
\hline \multicolumn{4}{|l|}{ At admission in all patients presenting with suspected AHF, the following diagnostic tests are recommended: } \\
\hline a. 12-lead ECG; & I & C & \\
\hline $\begin{array}{l}\text { b. chest X-ray to assess signs of pulmonary congestion and detect other cardiac or non-cardiac diseases that may } \\
\text { cause or contribute to the patient's symptoms; }\end{array}$ & I & C & \\
\hline $\begin{array}{l}\text { c. the following laboratory assessments in the blood: cardiac troponins, BUN (or urea), creatinine, electrolytes } \\
\text { (sodium, potassium), glucose, complete blood count, liver function tests and TSH. }\end{array}$ & I & C & \\
\hline $\begin{array}{l}\text { Echocardiography is recommended immediately in haemodynamically unstable AHF patients and within } 48 \text { hours when } \\
\text { cardiac structure and function are either not known or may have changed since previous studies. }\end{array}$ & $\mathbf{I}$ & C & \\
\hline
\end{tabular}

$\mathrm{AHF}=$ acute heart failure; $\mathrm{BNP}=\mathrm{B}$-type natriuretic peptide; $\mathrm{BUN}=$ blood urea nitrogen; $\mathrm{ECG}=$ electrocardiogram; $\mathrm{MR}$-proANP = mid-regional pro A-type natriuretic peptide; NT-proBNP = N-terminal pro-B type natriuretic peptide; $\mathrm{TSH}=$ thyroid-stimulating hormone

${ }^{\mathrm{a}}$ Class of recommendation.

bevel of evidence.

'Reference(s) supporting recommendations.

\subsection{Management}

AHF is a life-threatening medical condition, thus rapid transfer to the nearest hospital should be pursued, preferably to a site with a cardiology department and/or a coronary care/intensive care unit (CCU/ICU).

Early diagnosis is important in AHF. Therefore, all patients with suspected AHF should have a diagnostic workup and appropriate pharmacological and non-pharmacological treatment should be started promptly and in parallel.

Initial evaluation and continued non-invasive monitoring of the patient's vital cardiorespiratory functions, including pulse oximetry, blood pressure, respiratory rate and a continuous ECG instituted within minutes, is essential to evaluate whether ventilation, peripheral perfusion, oxygenation, heart rate and blood pressure are adequate. Urine output should also be monitored, although routine urinary catheterization is not recommended.

Patients with respiratory distress/failure or haemodynamic compromise should be triaged to a location where immediate respiratory and cardiovascular support can be provided (Figure 12.2).

\subsubsection{Identification of precipitants/causes leading to decompensation that needs urgent management}

The next step should comprise the identification of major precipitants/ causes leading to decompensation, which should be managed urgently to avoid further deterioration (Figure 12.2). These include the following:

- Acute coronary syndrome. Patients presenting with ACS should be managed according to the ESC guidelines on non-ST elevation ACS (NSTE-ACS) and STEMI. ${ }^{114,535}$ Coexistence of these two clinical conditions (ACS and AHF) always identifies a very-high-risk group where an immediate (i.e. $<2 \mathrm{~h}$ from hospital admission in patients with NSTEMI, analogous to STEMI management) invasive strategy with intent to perform revascularization is recommended, irrespective of ECG or biomarker findings. ${ }^{114,535}$ See below for patients presenting with persistent haemodynamic instability due to mechanical ACS complication.

- Hypertensive emergency. AHF precipitated by rapid and excessive increase in arterial blood pressure typically manifests as acute pulmonary oedema. A prompt reduction in blood pressure should be considered as a primary therapeutic target and initiated as soon as possible. Aggressive blood pressure reduction (in the range of $25 \%$ during the first few hours and cautiously thereafter) with i.v. vasodilators in combination with loop diuretics is recommended. ${ }^{317,536,537}$

- Rapid arrhythmias or severe bradycardia/conduction disturbance. Severe rhythm disturbances in patients with AHF and unstable conditions should be corrected urgently with medical therapy, electrical cardioversion or temporary pacing ${ }^{260,316,389}$ (see also Section 10.1 for AF management).

Electrical cardioversion is recommended if an atrial or ventricular arrhythmia is thought to be contributing to the patient's haemodynamic compromise in order to restore sinus rhythm and improve the patient's clinical condition.

Patients with AHF and incessant ventricular arrhythmias present a challenging scenario, as arrhythmias and haemodynamic instability operate in a vicious circle, perpetuating each other. In selected cases, immediate angiography (with resultant revascularization, if needed) and electrophysiological testing with radiofrequency ablation may be considered. ${ }^{260}$

- Acute mechanical cause underlying AHF. This may present as a mechanical complication of ACS (free wall rupture, ventricular septal defect, acute mitral regurgitation), chest trauma or cardiac intervention, or as acute native or prosthetic valve incompetence secondary to endocarditis, aortic dissection or thrombosis and comprise rare causes of obstruction (e.g. cardiac tumours). Echocardiography is essential for diagnosis, and treatment typically requires circulatory support with surgical or percutaneous intervention.

- Acute pulmonary embolism. When acute pulmonary embolism is confirmed as the cause of shock or hypotension, immediate specific treatment is recommended with primary reperfusion either with thrombolysis, catheter-based approach or surgical embolectomy. ${ }^{526}$ Patients presenting with acute pulmonary embolism should be managed according to the appropriate guidelines. ${ }^{526}$ 
Identification of acute aetiologies/precipitants with subsequent initiation of specific treatments should be done within the immediate phase of AHF management (initial 60-120 min) (Figure 12.2).

\subsubsection{Criteria for hospitalization in ward vs. intensive care/coronary care unit}

- Patients with persistent, significant dyspnoea or haemodynamic instability should be triaged to a location where immediate resuscitative support can be provided if needed.

- For high-risk patients (i.e. with persistent, significant dyspnoea, haemodynamic instability, recurrent arrhythmias, AHF and associated ACS), initial care should be provided in a high-dependency setting (ICU/CCU). Clinical risk algorithms developed to predict in-hospital mortality of patients with AHF can assist in determining which patients in the ED need the highest level of inpatient care. ${ }^{538,539}$

- The criteria for ICU/CCU admission include any of the following:

- need for intubation (or already intubated)
- signs/symptoms of hypoperfusion

- oxygen saturation $\left(\mathrm{SpO}_{2}\right)<90 \%$ (despite supplemental oxygen)

- use of accessory muscles for breathing, respiratory rate $>25 / \mathrm{min}$

- heart rate $<40$ or $>130 \mathrm{bpm}, \mathrm{SBP}<90 \mathrm{mmHg}$. ${ }^{540}$

- The remaining patients with AHF usually need hospitalization on an ordinary ward. Only a few patients admitted to the ED with AHF (mainly as exacerbation of HF symptoms with subtle signs of congestion) after a small dose of diuretics and some adjustments of oral therapy can be discharged directly home from the ED with advice to be clinically followed in an outpatient clinic.

- Step-down care from the ICU/CCU is dictated by clinical stabilization and resolution of morbid conditions. Further treatment will be continued with the involvement of a multidisciplinary team and discharge planning.

\subsubsection{Management of the early phase Oxygen therapy andlor ventilatory support}

\section{Recommendations for the management of patients with acute heart failure: oxygen therapy and ventilatory support}

\begin{tabular}{|c|c|c|c|}
\hline Recommendations & Class $^{a}$ & Level $^{b}$ & $\operatorname{Ref}^{c}$ \\
\hline Monitoring of transcutaneous arterial oxygen saturation $\left(\mathrm{SpO}_{2}\right)$ is recommended. & I & C & \\
\hline $\begin{array}{l}\text { Measurement of blood } \mathrm{pH} \text { and carbon dioxide tension (possibly including lactate) should be considered, especially in } \\
\text { patients with acute pulmonary oedema or previous history of COPD using venous blood. In patients with cardiogenic } \\
\text { shock arterial blood is preferable. }\end{array}$ & Ila & C & \\
\hline Oxygen therapy is recommended in patients with $\mathrm{AHF}$ and $\mathrm{SpO}_{2}<90 \%$ or $\mathrm{PaO}_{2}<60 \mathrm{mmHg}(8.0 \mathrm{kPa})$ to correct hypoxaemia. & I & C & \\
\hline $\begin{array}{l}\text { Intubation is recommended, if respiratory failure, leading to hypoxaemia }\left(\mathrm{PaO}_{2}<60 \mathrm{mmHg}(8.0 \mathrm{kPa})\right) \text {, hypercapnia } \\
\left(\mathrm{PaCO}_{2}>50 \mathrm{mmHg}(6.65 \mathrm{kPa})\right) \text { and acidosis }(\mathrm{pH}<7.35) \text {, cannot be managed non-invasively. }\end{array}$ & I & C & \\
\hline
\end{tabular}

$\mathrm{AHF}=$ acute heart failure; $\mathrm{BiPAP}=$ bilevel positive airway pressure; $\mathrm{COPD}=$ chronic obstructive pulmonary disease; $\mathrm{CPAP}=$ continuous positive airway pressure; $\mathrm{PaCO}{ }_{2}=$ partial pressure of carbon dioxide in arterial blood; $\mathrm{PaO}_{2}=$ partial pressure of oxygen in arterial blood; $\mathrm{SpO}_{2}=$ transcutaneous oxygen saturation.

${ }^{\mathrm{a} C}$ Class of recommendation.

bLevel of evidence.

${ }^{\mathrm{c}}$ Reference(s) supporting recommendations.

In AHF, oxygen should not be used routinely in non-hypoxaemic patients, as it causes vasoconstriction and a reduction in cardiac output. ${ }^{546,547}$ In COPD, hyperoxygenation may increase ventilationperfusion mismatch, suppressing ventilation and leading to hypercapnia. During oxygen therapy, acid-base balance and transcutaneous $\mathrm{SpO}_{2}$ should be monitored.

Non-invasive positive pressure ventilation includes both CPAP and bi-level positive pressure ventilation (PPV). Bi-level PPV also allows inspiratory pressure support that improves minute ventilation and is especially useful in patients with hypercapnia, most typically COPD patients.

Congestion affects lung function and increases intrapulmonary shunting, resulting in hypoxaemia. The fraction of inspired oxygen $\left(\mathrm{FiO}_{2}\right)$ should be increased up to $100 \%$ if necessary, according to $\mathrm{SpO}_{2}$, unless contraindicated. Hyperoxia, however, should be avoided. ${ }^{546,547}$ Non-invasive positive pressure ventilation reduces respiratory distress ${ }^{541-545}$ and may decrease intubation and mortality rates, ${ }^{543}$ although data regarding mortality are less conclusive. CPAP is a feasible technique in the pre-hospital setting, because it is simpler than pressure support positive end-expiratory pressure (PS-PEEP) and requires minimal training and equipment. On hospital arrival, patients who still show signs of respiratory distress should continue with non-invasive ventilation, preferably PS-PEEP, in case of acidosis and hypercapnia, particularly in those with a previous history of COPD or signs of fatigue. $^{540}$

Caution should be exercised with regard to side effects of anaesthetic drugs, among which propofol can induce hypotension and have cardiodepressive side effects. In contrast, midazolam may have fewer cardiac side effects and thus is preferred in patients with AHF or cardiogenic shock.

A management algorithm for patients with AHF based on the clinical profile during an early phase is presented in Figure 12.3 . 


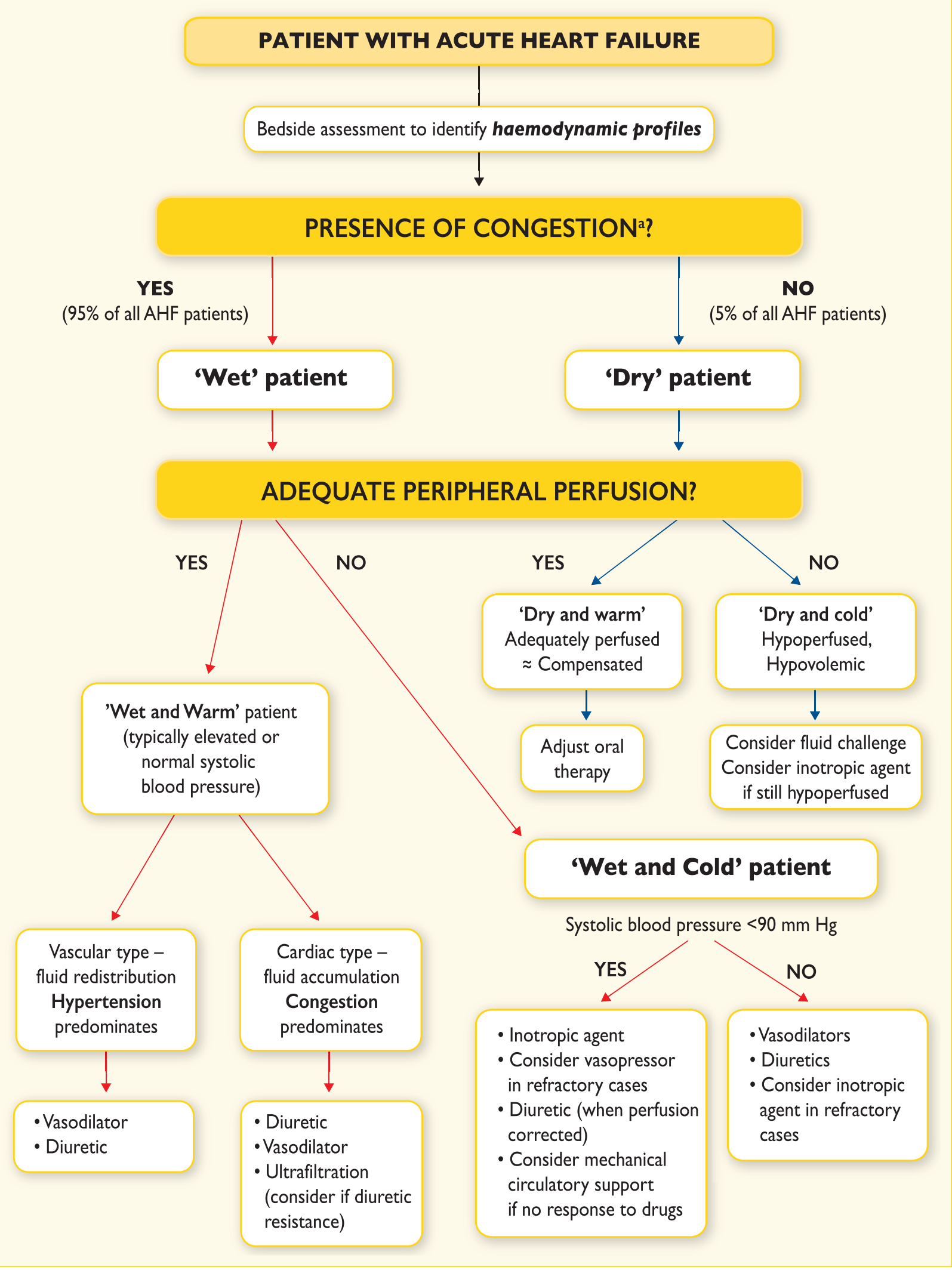

Figure I 2.3 Management of patients with acute heart failure based on clinical profile during an early phase

a Symptoms/signs of congestion: orthopnoea, paroxysmal nocturnal dyspnoea, breathlessness, bi-basilar rales, an abnormal blood pressure response to the Valsalva maneuver (left-sided); symptoms of gut congestion, jugular venous distension, hepatojugular reflux, hepatomegaly, ascites, and peripheral oedema (right-sided). 


\section{Pharmacological therapy}

Recommendations for the management of patients with acute heart failure: pharmacotherapy

\begin{tabular}{|c|c|c|c|}
\hline Recommendations & Class $^{a}$ & Level $^{b}$ & $\operatorname{Ref}^{c}$ \\
\hline \multicolumn{4}{|l|}{ Diuretics } \\
\hline $\begin{array}{l}\text { Intravenous loop diuretics are recommended for all patients with AHF admitted with signs/symptoms of fluid overload to } \\
\text { improve symptoms. It is recommended to regularly monitor symptoms, urine output, renal function and electrolytes during } \\
\text { use of i.v. diuretics. }\end{array}$ & I & C & \\
\hline $\begin{array}{l}\text { In patients with new-onset AHF or those with chronic, decompensated HF not receiving oral diuretics the initial } \\
\text { recommended dose should be } 20-40 \mathrm{mg} \text { i.v. furosemide (or equivalent); for those on chronic diuretic therapy, initial i.v. } \\
\text { dose should be at least equivalent to oral dose. }\end{array}$ & I & $\mathbf{B}$ & 540,548 \\
\hline $\begin{array}{l}\text { It is recommended to give diuretics either as intermittent boluses or as a continuous infusion, and the dose and duration } \\
\text { should be adjusted according to patients' symptoms and clinical status. }\end{array}$ & I & B & 548 \\
\hline $\begin{array}{l}\text { Combination of loop diuretic with either thiazide-type diuretic or spironolactone may be considered in patients with } \\
\text { resistant oedema or insufficient symptomatic response. }\end{array}$ & Ilb & C & 549 \\
\hline \multicolumn{4}{|l|}{ Vasodilators } \\
\hline $\begin{array}{l}\text { i.v. vasodilators should be considered for symptomatic relief in AHF with SBP }>90 \mathrm{mmHg} \text { (and without symptomatic } \\
\text { hypotension). } \\
\text { Symptoms and blood pressure should be monitored frequently during administration of i.v. vasodilators. }\end{array}$ & Ila & B & $\begin{array}{c}537, \\
550-555\end{array}$ \\
\hline $\begin{array}{l}\text { In patients with hypertensive AHF, i.v. vasodilators should be considered as initial therapy to improve symptoms and reduce } \\
\text { congestion. }\end{array}$ & Ila & B & $\begin{array}{l}537, \\
551-554\end{array}$ \\
\hline \multicolumn{4}{|l|}{ Inotropic agents - dobutamine, dopamine, levosimendan, phosphodiesterase III (PDE III) inhibitors } \\
\hline $\begin{array}{l}\text { Short-term, i.v. infusion of inotropic agents may be considered in patients with hypotension (SBP }<90 \mathrm{mmHg} \text { ) and/or signs/ } \\
\text { symptoms of hypoperfusion despite adequate filling status, to increase cardiac output, increase blood pressure, improve } \\
\text { peripheral perfusion and maintain end-organ function. }\end{array}$ & Ilb & C & \\
\hline $\begin{array}{l}\text { An intravenous infusion of levosimendan or a PDE III inhibitor may be considered to reverse the effect of beta-blockade } \\
\text { if beta-blockade is thought to be contributing to hypotension with subsequent hypoperfusion. }\end{array}$ & Ilb & C & \\
\hline $\begin{array}{l}\text { Inotropic agents are not recommended unless the patient is symptomatically hypotensive or hypoperfused because of } \\
\text { safety concern. }\end{array}$ & III & A & 556,557 \\
\hline $\begin{array}{l}\text { A vasopressor (norepinephrine preferably) may be considered in patients who have cardiogenic shock, despite treatment } \\
\text { with another inotrope, to increase blood pressure and vital organ perfusion. }\end{array}$ & Illb & B & 558 \\
\hline $\begin{array}{l}\text { It is recommended to monitor ECG and blood pressure when using inotropic agents and vasopressors, as they can cause } \\
\text { arrhythmia, myocardial ischaemia, and in the case of levosimendan and PDE III inhibitors also hypotension. }\end{array}$ & I & C & $\begin{array}{c}540, \\
559-563\end{array}$ \\
\hline In such cases intra-arterial blood pressure measurement may be considered. & Illb & C & \\
\hline \multicolumn{4}{|l|}{ Thrombo-embolism prophylaxis } \\
\hline $\begin{array}{l}\text { Thrombo-embolism prophylaxis (e.g. with LMWH) is recommended in patients not already anticoagulated and with no } \\
\text { contra-indication to anticoagulation, to reduce the risk of deep venous thrombosis and pulmonary embolism. }\end{array}$ & $\mathbf{I}$ & B & 564 \\
\hline \multicolumn{4}{|l|}{ Other drugs } \\
\hline \multicolumn{4}{|l|}{ For acute control of the ventricular rate In patients with atrial fibrillation: } \\
\hline a. digoxin and/or beta-blockers should be considered as the first-line therapy. ${ }^{d}$ & Ila & C & \\
\hline b. amiodarone may be considered. & Illb & B & $565-567$ \\
\hline $\begin{array}{l}\text { Opiates may be considered for cautious use to relieve dyspnoea and anxiety in patients with severe dyspnoea but nausea } \\
\text { and hypopnea may occur. }\end{array}$ & IIb & B & 568,569 \\
\hline
\end{tabular}

$\mathrm{AHF}=$ acute heart failure; $\mathrm{ECG}=$ electrocardiogram; $\mathrm{HF}=$ heart failure; i.v. = intravenous; $\mathrm{LMWH}=$ low molecular weight heparin; $\mathrm{SBP}=$ systolic blood pressure. ${ }^{a}$ Class of recommendation.

bLevel of evidence.

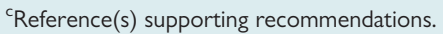

${ }^{d}$ Beta-blockers should be used cautiously, if the patient is hypotensive. 


\section{Diuretics}

Diuretics are a cornerstone in the treatment of patients with AHF and signs of fluid overload and congestion. Diuretics increase renal salt and water excretion and have some vasodilatory effect. In patients with AHF and signs of hypoperfusion, diuretics should be avoided before adequate perfusion is attained.

The initial approach to congestion management involves i.v. diuretics with the addition of vasodilators for dyspnoea relief if blood pressure allows. To enhance diuresis or overcome diuretic resistance, options include dual nephron blockade by loop diuretics (i.e. furosemide or torasemide) with thiazide diuretics or natriuretic doses of MRAs. ${ }^{570,571}$ However, this combination requires careful monitoring to avoid hypokalaemia, renal dysfunction and hypovolaemia.

Data defining optimal dosing, timing and method of delivery are incomplete. In the 'high-dose' arm of the DOSE study, administration of furosemide at 2.5 times the previous oral dose resulted in greater improvement in dyspnoea, larger weight change and fluid loss at the cost of transient worsening in renal function. ${ }^{548}$ In AHF, i.v. furosemide is the most commonly used first-line diuretic. The dose should be limited to the smallest amount to provide adequate clinical effect and modified according to previous renal function and previous dose of diuretics. The initial i.v. dose should be at least equal to the preexisting oral dose used at home. Consequently, patients with newonset AHF or those with chronic HF without a history of renal failure and previous use of diuretics may respond to i.v. boluses of $20-$ $40 \mathrm{mg}$, whereas those with previous use of diuretics usually require higher doses. A bolus of 10-20 mg i.v. torasemide may be considered as an alternative.

\section{Vasodilators}

Intravenous vasodilators (Table 12.4) are the second most often used agents in AHF for symptomatic relief; however, there is no robust evidence confirming their beneficial effects.

They have dual benefit by decreasing venous tone (to optimize preload) and arterial tone (decrease afterload). Consequently, they may also increase stroke volume. Vasodilators are especially useful in patients with hypertensive AHF, whereas in those with $\mathrm{SBP}<90 \mathrm{mmHg}$ (or with symptomatic hypotension) they should be avoided. Dosing should be carefully controlled to avoid excessive decreases in blood pressure, which is related to poor outcome. Vasodilators should be used with caution in patients with significant mitral or aortic stenosis.
Use of an inotrope (Table 12.5) should be reserved for patients with a severe reduction in cardiac output resulting in compromised vital organ perfusion, which occurs most often in hypotensive AHF. Inotropic agents are not recommended in cases of hypotensive AHF where the underlying cause is hypovolaemia or other potentially correctable factors before elimination of these causes. Levosimendan is preferable over dobutamine to reverse the effect of betablockade if beta-blockade is thought to be contributing to hypoperfusion. ${ }^{572}$ However, levosimendan is a vasodilator, thus it is not suitable for treatment of patients with hypotension (SBP $<85 \mathrm{mmHg}$ ) or cardiogenic shock unless in combination with other inotropes or vasopressors. ${ }^{559,573,574}$ Inotropes, especially those with adrenergic mechanisms, can cause sinus tachycardia and may induce myocardial ischaemia and arrhythmias, thus ECG monitoring is required. There is long-standing concern that they may increase mortality, which

\section{Table I2.5 Positive inotropes and/or vasopressors} used to treat acute heart failure

\begin{tabular}{|c|c|c|}
\hline Vasodilator & Bolus & Infusion rate \\
\hline Dobutamine $^{\mathrm{a}}$ & No & $2-20 \mu \mathrm{g} / \mathrm{kg} / \mathrm{min}($ beta +$)$ \\
\hline \multirow[t]{2}{*}{ Dopamine } & No & 3-5 $\mu \mathrm{g} / \mathrm{kg} / \mathrm{min}$; inotropic (beta+) \\
\hline & & $\begin{array}{l}>5 \mu g / k g / m i n:(\text { beta }+) \\
\text { vasopressor }(\text { alpha }+)\end{array}$ \\
\hline Milrinone $e^{a, b}$ & $\begin{array}{l}25-75 \mu g / \mathrm{kg} \\
\text { over } 10-20 \mathrm{~min}\end{array}$ & $0.375-0.75 \mu \mathrm{g} / \mathrm{kg} / \mathrm{min}$ \\
\hline Enoximone $^{\mathrm{a}}$ & $\begin{array}{l}0.5-1.0 \mathrm{mg} / \mathrm{kg} \\
\text { over } 5-10 \mathrm{~min}\end{array}$ & $5-20 \mu g / k g / m i n$ \\
\hline Levosimendan $^{\mathrm{a}}$ & $\begin{array}{l}12 \mu \mathrm{g} / \mathrm{kg} \text { over } \\
10 \mathrm{~min} \text { (optional) }\end{array}$ & $\begin{array}{l}0.1 \mu \mathrm{g} / \mathrm{kg} / \mathrm{min} \text {, which can be } \\
\text { decreased to } 0.05 \text { or increased } \\
\text { to } 0.2 \mu \mathrm{g} / \mathrm{kg} / \mathrm{min}\end{array}$ \\
\hline Norepinephrine & No & $0.2-1.0 \mu \mathrm{g} / \mathrm{kg} / \mathrm{min}$ \\
\hline Epinephrine & $\begin{array}{l}\text { Bolus: I mg } \\
\text { can be given } \\
\text { i.v. during } \\
\text { resuscitation, } \\
\text { repeated every } \\
3-5 \text { min }\end{array}$ & $0.05-0.5 \mu \mathrm{g} / \mathrm{kg} / \mathrm{min}$ \\
\hline
\end{tabular}

i.v. $=$ intravenous.

${ }^{\mathrm{a} A l s o}$ a vasodilator.

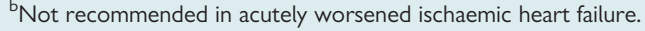

'Bolus not recommended in hypotensive patients.

Table I 2.4 Intravenous vasodilators used to treat acute heart failure

\begin{tabular}{|l|l|l|l|}
\hline Vasodilator & Dosing & Main side effects & Other \\
\hline Nitroglycerine & Start with $10-20 \mu g /$ min, increase up to $200 \mu \mathrm{g} / \mathrm{min}$ & Hypotension, headache & Tolerance on continuous use \\
\hline Isosorbide dinitrate & Start with $1 \mathrm{mg} / \mathrm{h}$, increase up to $10 \mathrm{mg} / \mathrm{h}$ & Hypotension, headache & Tolerance on continuous use \\
\hline Nitroprusside & Start with $0.3 \mu \mathrm{g} / \mathrm{kg} / \mathrm{min}$ and increase up to $5 \mu \mathrm{g} / \mathrm{kg} / \mathrm{min}$ & Hypotension, isocyanate toxicity & Light sensitive \\
\hline Nesiritide & Bolus $2 \mu \mathrm{g} / \mathrm{kg}+$ infusion $0.01 \mu \mathrm{g} / \mathrm{kg} / \mathrm{min}$ & Hypotension & \\
\hline
\end{tabular}


derives from studies in which intermittent or continuous infusions of inotropes were given. ${ }^{559-563,575}$ In any case, inotropes have to be used with caution starting from rather low doses and up-titrating with close monitoring.

\section{Vasopressors}

Drugs with prominent peripheral arterial vasoconstrictor action such as norepinephrine or dopamine in higher doses $(>5 \mu \mathrm{g} / \mathrm{kg} /$ min) are given to patients with marked hypotension. These agents are given to raise blood pressure and redistribute blood to the vital organs. However, this is at the expense of an increase in LV afterload.

Dopamine was compared with norepinephrine in the treatment of various shock patients. A subgroup analysis suggested that norepinephrine would have fewer side effects and lower mortality. ${ }^{558}$ Epinephrine (adrenaline) should be restricted to patients with persistent hypotension despite adequate cardiac filling pressures and the use of other vasoactive agents, as well as for resuscitation protocols. $^{576}$

\section{Thromboembolism prophylaxis}

Thromboembolism prophylaxis with heparin or another anticoagulant is recommended unless contraindicated or unnecessary (because of existing treatment with oral anticoagulants).

\section{Digoxin}

Digoxin is mostly indicated in patients with $\mathrm{AF}$ and rapid ventricular rate $(>110 \mathrm{bpm})$ and given in boluses of $0.25-0.5 \mathrm{mg}$ i.v. if not used previously $(0.0625-0.125 \mathrm{mg}$ may be an adequate dose in patients with moderate to severe renal dysfunction). However, in patients with co-morbidities or other factors affecting digoxin metabolism (including other drugs) and/or the elderly, the maintenance dose may be difficult to estimate theoretically, and in this situation it should be established empirically, based on the measurements of digoxin concentration in peripheral blood.

\section{Vasopressin antagonists}

Vasopressin antagonists such as tolvaptan block the action of arginine vasopressin (AVP) at the $V_{2}$ receptor in renal tubules and promote aquaresis. Tolvaptan may be used to treat patients with volume overload and resistant hyponatraemia (thirst and dehydration are recognized adverse effects). ${ }^{577}$

\section{Opiates}

Opiates relieve dyspnoea and anxiety. In AHF, routine use of opiates is not recommended and they may only be cautiously considered in patients with severe dyspnoea, mostly with pulmonary oedema. Dose-dependent side effects include nausea, hypotension, bradycardia and respiratory depression (potentially increasing the need for invasive ventilation). There are controversies regarding the potentially elevated mortality risk in patients receiving morphine. ${ }^{568,569}$

\section{Anxiolytics and sedatives}

Anxiolytics or sedatives may be needed in a patient with agitation or delirium. Cautious use of benzodiazepines (diazepam or lorazepam) may be the safest approach.

\section{Device therapy}

\section{Recommendations regarding renal replacement therapy in patients with acute heart failure}

\begin{tabular}{|l|c|c|c|}
\hline Recommendations & Class $^{\text {a }}$ & Level $^{\text {b }}$ & Ref $^{\text {C }}$ \\
\hline $\begin{array}{l}\text { Ultrafiltration may be considered for } \\
\text { patients with refractory congestion, } \\
\text { who failed to respond to diuretic- } \\
\text { based strategies. }\end{array}$ & Ilb & B & 578-580 \\
\hline $\begin{array}{l}\text { Renal replacement therapy should } \\
\text { be considered in patients with } \\
\text { refractory volume overload and } \\
\text { acute kidney injury. }\end{array}$ & Ila & C & \\
\hline
\end{tabular}

${ }^{\mathrm{a}}$ Class of recommendation

bLevel of evidence.

'Reference(s) supporting recommendations.

\section{Renal replacement therapy}

Ultrafiltration involves the removal of plasma water across a semipermeable membrane in response to a transmembrane pressure gradient. There is no evidence favouring ultrafiltration over loop diuretics as first-line therapy in patients with $A H F .{ }^{571,578} \mathrm{At}$ the present time, routine use of ultrafiltration is not recommended and should be confined to patients who fail to respond to diureticbased strategies.

The following criteria may indicate the need for initiation of renal replacement therapy in patients with refractory volume overload: oliguria unresponsive to fluid resuscitation measures, severe hyperkalaemia $\left(\mathrm{K}^{+}>6.5 \mathrm{mmol} / \mathrm{L}\right)$, severe acidaemia $(\mathrm{pH}<7.2)$, serum urea level $>25 \mathrm{mmol} / \mathrm{L}(150 \mathrm{mg} / \mathrm{dL})$ and serum creatinine $>300$ $\mu \mathrm{mol} / \mathrm{L}(>3.4 \mathrm{mg} / \mathrm{dL})$.

\section{Mechanical assist devices \\ Intra-aortic balloon pump}

The conventional indications for an intra-aortic balloon pump (IABP) are to support the circulation before surgical correction of specific acute mechanical problems (e.g. interventricular septal rupture and acute mitral regurgitation), during severe acute myocarditis and in selected patients with acute myocardial ischaemia or infarction before, during and after percutaneous or surgical revascularization. There is no good evidence that an IABP is of benefit in other causes of cardiogenic shock (for details see below).

\section{Ventricular assist devices}

Ventricular assist devices and other forms of mechanical circulatory support (MCS) may be used as a 'bridge to decision' or longer term in selected patients (see Section 13).

\section{Other interventions}

In patients with AHF and pleural effusion, pleurocentesis with fluid evacuation may be considered if feasible in order to alleviate dyspnoea.

In patients with ascites, ascitic paracentesis with fluid evacuation may be considered in order to alleviate symptoms. This procedure, through reduction in intra-abdominal pressure, may also partially normalize the transrenal pressure gradient, thus improving renal filtration. $^{581}$ 


\subsubsection{Management of patients with cardiogenic shock}

Cardiogenic shock is defined as hypotension (SBP $<90 \mathrm{mmHg}$ ) despite adequate filling status with signs of hypoperfusion (Table 12.2). The pathogenetic scenarios of cardiogenic shock range from lowoutput advanced end-stage chronic HF to acute-onset de novo cardiogenic shock most often caused by STEMI, but also by various aetiologies other than ACS. A patient in cardiogenic shock should undergo immediate comprehensive assessment. ECG and echocardiography are required immediately in all patients with suspected cardiogenic shock. In patients with cardiogenic shock complicating ACS, an immediate coronary angiography is recommended (within $2 \mathrm{~h}$ from hospital admission) with an intent to perform coronary revascularization. ${ }^{114,535}$ Invasive monitoring with an arterial line should be also considered.

There is no agreement on the optimal method of haemodynamic monitoring in assessing and treating patients in cardiogenic shock, including pulmonary artery catheterization.

Pharmacologic therapy aims to improve organ perfusion by increasing cardiac output and blood pressure. After fluid challenge, pharmacologic management consists of an inotropic agent and a vasopressor as needed. Treatment is guided by the continuous monitoring of organ perfusion and haemodynamics. Pulmonary artery catheterization may be considered. As a vasopressor, norepinephrine is recommended when mean arterial pressure needs pharmacologic support. Dobutamine is the most commonly used adrenergic inotrope. Levosimendan may also be used in combination with a vasopressor. ${ }^{582,583}$ Levosimendan infusion in cardiogenic shock following AMI on top of dobutamine and norepinephrine improved cardiovascular haemodynamics without leading to hypotension. ${ }^{582,583}$ PDE3 inhibitors may be another option, especially in non-ischaemic patients. ${ }^{561,584}$

However, rather than combining several inotropes, device therapy has to be considered when there is an inadequate response. Recently the IABP-SHOCK II trial showed that the use of an IABP did not improve outcomes in patients suffering from AMI and cardiogenic shock. ${ }^{585,586}$ Therefore, routine use of an IABP cannot be recommended.

\subsection{Management of evidence-based oral therapies}
Recommendations regarding oral evidence-based disease-modifying therapies in patients with acute heart failure

\begin{tabular}{|l|c|c|}
\hline Recommendations & Class $^{\text {a }}$ & Level $^{\mathbf{b}}$ \\
\hline $\begin{array}{l}\text { In case of worsening of chronic HFrEF, every } \\
\text { attempt should be made to continue evidence- } \\
\text { based, disease-modifying therapies, in the } \\
\text { absence of haemodynamic instability or contra- } \\
\text { indications. }\end{array}$ & I & C \\
\hline $\begin{array}{l}\text { In the case of de novo HFrEF, every attempt } \\
\text { should be made to initiate these therapies after } \\
\text { haemodynamic stabilization. }\end{array}$ & I & C \\
\hline
\end{tabular}

$\mathrm{AHF}=$ acute heart failure; $\mathrm{HFrEF}=$ heart failure with reduced ejection fraction.

${ }^{\mathrm{a}}$ Class of recommendation.

bevel of evidence.

Oral disease-modifying HF therapy should be continued on admission with AHF, except in the presence of haemodynamic instability (symptomatic hypotension, hypoperfusion, bradycardia), hyperkalaemia or severely impaired renal function. In these cases, the daily dosage of oral therapy may be reduced or stopped temporarily until the patient is stabilized. In particular, beta-blockers can be safely continued during AHF presentations except in cardiogenic shock. A recent meta-analysis demonstrated that discontinuation of beta-blockers in patients hospitalized with AHF was associated with significantly increased in-hospital mortality, shortterm mortality and the combined endpoint of short-term rehospitalization or mortality. ${ }^{587}$

\section{Recommendations regarding management of patients with cardiogenic shock}

\begin{tabular}{|c|c|c|c|}
\hline Recommendations & Class $^{\mathrm{a}}$ & Level $^{\mathrm{b}}$ & $\operatorname{Ref}^{c}$ \\
\hline In all patients with suspected cardiogenic shock, immediate ECG and echocardiography are recommended. & $\mathbf{I}$ & C & \\
\hline $\begin{array}{l}\text { All patients with cardiogenic shock should be rapidly transferred to a tertiary care center which has a } 24 / 7 \text { service of cardiac } \\
\text { catheterization, and a dedicated ICU/CCU with availability of short-term mechanical circulatory support. }\end{array}$ & $\mathbf{I}$ & C & \\
\hline $\begin{array}{l}\text { In patients with cardiogenic shock complicating ACS an immediate coronary angiography is recommended (within } 2 \text { hours } \\
\text { from hospital admission) with an intent to perform coronary revascularization. }\end{array}$ & I & C & \\
\hline Continous ECG and blood pressure monitoring are recommended. & I & C & \\
\hline Invasive monitoring with an arterial line is recommended. & I & C & \\
\hline $\begin{array}{l}\text { Fluid challenge (saline or Ringer's lactate, }>200 \mathrm{ml} / / 5-30 \mathrm{~min} \text { ) is recommended as the first-line treatment if there is no sign of } \\
\text { overt fluid overload. }\end{array}$ & $\mathbf{I}$ & c & \\
\hline Intravenous inotropic agents (dobutamine) may be considered to increase cardiac output. & Ilb & C & \\
\hline $\begin{array}{l}\text { Vasopressors (norepinephrine preferable over dopamine) may be considered if there is a need to maintain SBP in the } \\
\text { presence of persistent hypoperfusion. }\end{array}$ & Illb & B & 558 \\
\hline IABP is not routinely recommended in cardiogenic shock. & III & B & 585,586 \\
\hline $\begin{array}{l}\text { Short-term mechanical circulatory support may be considered in refractory cardiogenic shock depending on patient age, } \\
\text { comorbidities and neurological function. }\end{array}$ & Ilb & C & \\
\hline
\end{tabular}

$\mathrm{ACS}=$ acute coronary syndrome; $\mathrm{CCU}=$ coronary care unit; $\mathrm{ECG}=$ electrocardiogram; IABP $=$ intra-aortic balloon pump; ICU $=$ intensive care unit; $\mathrm{SBP}=$ systolic blood pressure.

${ }^{\mathrm{a}}$ Class of recommendation.

bevel of evidence.

${ }^{c}$ Reference(s) supporting recommendations. 


\subsection{Monitoring of clinical status of patients hospitalized due to acute heart failure}

\section{Recommendations regarding monitoring of clinical status of patients hospitalized due to acute heart failure

\begin{tabular}{|l|c|c|}
\hline Recommendations & Class & ' \\
\hline $\begin{array}{l}\text { Sevel } \\
\text { Standard non-invasive monitoring of heart rate, } \\
\text { rhythm, respiratory rate, oxygen saturation and } \\
\text { blood pressure is recommended. }\end{array}$ & I & C \\
\hline $\begin{array}{l}\text { It is recommended that patients should be } \\
\text { weighed daily and have an accurate fluid balance } \\
\text { chart completed. }\end{array}$ & I & C \\
\hline $\begin{array}{l}\text { It is recommended to evaluate signs and } \\
\text { symptoms relevant to HF (e.g. dyspnoea, } \\
\text { pulmonary rales, peripheral oedema, weight) } \\
\text { daily to assess correction of fluid overload. }\end{array}$ & I & C \\
\hline $\begin{array}{l}\text { Frequent, often daily,measurement of } \\
\text { renal function (blood urea, creatinine) and } \\
\text { electrolytes (potassium, sodium) during } \\
\text { i.v. therapy and when renin-angiotensin- } \\
\text { aldosterone system antagonists are initiated is } \\
\text { recommended. }\end{array}$ & I & C \\
\hline $\begin{array}{l}\text { Intra-arterial line should be considered in } \\
\text { patients with hypotension and persistent } \\
\text { symptoms despite treatment. }\end{array}$ & Ila & C \\
\hline $\begin{array}{l}\text { Pulmonary artery catheter may be considered } \\
\text { in patients who, despite pharmacological } \\
\text { treatment present refractory symptoms } \\
\text { (particularly with hypotension and } \\
\text { hypoperfusion). }\end{array}$ & & \\
\hline
\end{tabular}

$\mathrm{HF}=$ heart failure; i.v. $=$ intravenous.

${ }^{a}$ Class of recommendation.

bevel of evidence.

Patients should be weighed daily and an accurate fluid balance chart should be maintained. Renal function should preferably be monitored with daily measurement of BUN/urea, creatinine and electrolytes. Routine use of a urinary catheter is not recommended.

Renal function is commonly impaired at admission, but may improve or deteriorate with diuresis. Routine monitoring of pulse, respiratory rate and blood pressure should continue. There is no study showing the usefulness of invasive haemodynamic monitoring in patients with AHF excluding those with cardiogenic shock. There is evidence that measuring NPs during the hospital admission may help with discharge planning. Patients whose NP concentrations fall during admission have lower cardiovascular mortality and readmission rates at 6 months. ${ }^{588-590}$

\subsection{Criteria for discharge from hospital and follow-up in high-risk period}

Patients admitted with AHF are medically fit for discharge ${ }^{552}$

- when haemodynamically stable, euvolaemic, established on evidence-based oral medication and with stable renal function for at least 24 hours before discharge;
- once provided with tailored education and advice about self-care.

Patients should preferably be

- enrolled in a disease management programme; follow-up plans must be in place prior to discharge and clearly communicated to the primary care team;

- reviewed by their general practitioner within 1 week of discharge;

- seen by the hospital cardiology team within 2 weeks of discharge if feasible.

Patients with chronic HF should be followed up within a multiprofessional HF service. Pre- and post-discharge management should follow the standards of care of the HFA. ${ }^{540,591,592}$

\subsection{Goals of treatment during the different stages of management of acute heart failure}

During the treatment of patients with AHF, one can distinguish subsequent stages that require different therapeutic approaches (described in the previous sections of this chapter). Importantly, the goals of treatment during the different stages of management of patients with AHF also differ, and are summarized in Table 12.6.

\section{Table I 2.6 Goals of treatment in acute heart failure}

\begin{tabular}{|l|}
\hline Immediate (ED/ICU/CCU) \\
\hline Improve haemodynamics and organ perfusion. \\
\hline Restore oxygenation. \\
\hline Alleviate symptoms. \\
\hline Limit cardiac and renal damage. \\
\hline Prevent thrombo-embolism. \\
\hline Minimize ICU length of stay. \\
\hline Intermediate (in hospital) \\
\hline Identify aetiology and relevant co-morbidities. \\
\hline $\begin{array}{l}\text { Titrate therapy to control symptoms and congestion and optimize blood } \\
\text { pressure. }\end{array}$ \\
\hline Initiate and up-titrate disease-modifying pharmacological therapy. \\
\hline Consider device therapy in appropriate patients. \\
\hline Pre-discharge and long-term management \\
\hline $\begin{array}{l}\text { Develop a careplan that provides: } \\
\text { oA schedule for up-titration and monitoring of pharmacological therapy. } \\
\text { o Need and timing for review for device therapy. } \\
\text { oWho will see the patient for follow-up and when. }\end{array}$ \\
\hline $\begin{array}{l}\text { Enrol in disease management programme, educate, and initiate } \\
\text { appropriate lifestyle adjustments. }\end{array}$ \\
\hline Prevent early readmission. \\
\hline Improve symptoms, quality of life, and survival. \\
\hline
\end{tabular}

$\mathrm{CCU}=$ coronary care unit; $\mathrm{ED}=$ emergency department; $\mathrm{ICU}=$ intensive care unit. 


\section{Mechanical circulatory support and heart transplantation}

\subsection{Mechanical circulatory support}

For patients with either chronic or acute HF who cannot be stabilized with medical therapy, MCS systems can be used to unload the failing ventricle and maintain sufficient end-organ perfusion. Patients in acute cardiogenic shock are initially treated with short-term assistance using extracorporeal, non-durable life support systems so that more definitive therapy may be planned. Patients with chronic, refractory HF despite medical therapy can be treated with a permanent implantable left ventricular assist device (LVAD). Table 13.1 lists the current indications for the use of mechanical circulatory assist devices. ${ }^{593}$

\subsubsection{Mechanical circulatory support in acute heart failure}

To manage patients with AHF or cardiogenic shock (INTERMACS level 1), short-term mechanical support systems, including percutaneous cardiac support devices, extracorporeal life support (ECLS) and extracorporeal membrane oxygenation (ECMO) may be used to support patients with left or biventricular failure until cardiac and other organ function have recovered. Typically the use of these devices is restricted to a few days to weeks. The Survival After Veno-arterial ECMO (SAVE) score can help to predict survival

Table I3.I Terms describing various indications for mechanical circulatory support

\begin{tabular}{|l|l|}
\hline $\begin{array}{l}\text { Bridge to } \\
\text { decision (BTD)/ } \\
\text { Bridge to } \\
\text { bridge (BTB) }\end{array}$ & $\begin{array}{l}\text { Use of short-term MCS (e.g. ECLS or ECMO) } \\
\text { in patients with cardiogenic shock until } \\
\text { haemodynamics and end-organ perfusion are } \\
\text { stabilized, contra-indications for long-term MCS } \\
\text { are excluded (brain damage after resuscitation) } \\
\text { and additional therapeutic options including } \\
\text { long-term VAD therapy or heart transplant can } \\
\text { be evaluated. }\end{array}$ \\
\hline $\begin{array}{l}\text { Bridge to } \\
\text { candidacy } \\
\text { (BTC) }\end{array}$ & $\begin{array}{l}\text { Use of MCS (usually LVAD) to improve end-organ } \\
\text { function in order to make an ineligible patient } \\
\text { eligible for heart transplantation. }\end{array}$ \\
\hline $\begin{array}{l}\text { Bridge to } \\
\text { transplantation } \\
\text { (BTT) }\end{array}$ & $\begin{array}{l}\text { Use of MCS (LVAD or BiVAD) to keep patient } \\
\text { alive who is otherwise at high risk of death before } \\
\text { transplantation until a donor organ becomes } \\
\text { available. }\end{array}$ \\
\hline $\begin{array}{l}\text { Bridge to } \\
\text { recovery (BTR) }\end{array}$ & $\begin{array}{l}\text { Use of MCS (typically LVAD) to keep patient } \\
\text { alive until cardiac function recovers sufficiently to } \\
\text { remove MCS. }\end{array}$ \\
\hline $\begin{array}{l}\text { Destination } \\
\text { therapy (DT) }\end{array}$ & $\begin{array}{l}\text { Long-term use of MCS (LVAD) as an alternative } \\
\text { to transplantation in patients with end-stage HF } \\
\text { ineligible for transplantation or long-term waiting } \\
\text { for heart transplantation. }\end{array}$ \\
\hline
\end{tabular}

BiVAD = biventricular assist device; $\mathrm{BTB}=$ bridge to bridge; $\mathrm{BTC}=$ bridge to candidacy; $\mathrm{BTD}=$ bridge to decision; $\mathrm{BTR}=$ bridge to recovery; $\mathrm{BTT}=$ bridge to transplantation; DT = destination therapy; ECLS = extracorporeal life support; $\mathrm{ECMO}=$ extracorporeal membrane oxygenation; $\mathrm{HF}=$ heart failure; $\mathrm{LVAD}=$ left ventricular assist device; $M C S=$ mechanical circulatory support; VAD = ventricular assist device. for patients receiving ECMO for refractory cardiogenic shock (online calculator at http://www.save-score.com). ${ }^{594}$

In addition, MCS systems, particularly ECLS and ECMO, can be used as a 'bridge to decision' (BTD) in patients with acute and rapidly deteriorating HF or cardiogenic shock to stabilize haemodynamics, recover end-organ function and allow for a full clinical evaluation for the possibility of either heart transplant or a more durable MCS device. ${ }^{595}$

Evidence regarding the benefits of temporary percutaneous MCS in patients not responding to standard therapy, including inotropes, is limited. In a meta-analysis of three randomized clinical trials comparing a percutaneous MCS vs. IABP in a total of 100 patients in cardiogenic shock, percutaneous MCS appeared safe and demonstrated better haemodynamics, but did not improve 30-day mortality and was associated with more bleeding complications. ${ }^{596}$ In a randomized trial on high-risk $\mathrm{PCl}$ in patients with impaired $\mathrm{LV}$ function (PROTECT II trial), the 30-day incidence of major adverse events was not different for patients with IABP or a haemodynamic support device. ${ }^{597}$ Based on these results, temporary percutaneous MCS cannot be recommended as a proven or efficacious treatment for acute cardiogenic shock. In selected patients it may serve as a bridge to definite therapy. A difficult decision to withdraw MCS may need to be made when the patient has no potential for cardiac recovery and is not eligible for longer-term MCS support or heart transplant.

\subsubsection{Mechanical circulatory support in end-stage chronic heart failure}

Heart transplantation has always been a limited therapeutic option for patients with end-stage chronic HF. The increasing number of patients with refractory, chronic HF and the declining willingness for organ donation have resulted in expanded waiting lists and prolonged waiting times for patients listed for heart transplantation (median 16 months in the region covered by Eurotransplant). ${ }^{598}$ More than $60 \%$ of patients are transplanted in high-urgency status, leaving little chance for patients listed for less urgent transplantation. Three times more patients are listed for heart transplantation annually than are actually transplanted, and the mortality rate on the Eurotransplant waiting list in 2013 was $21.7 \%{ }^{598}$

More recent data suggest that patients with ongoing LVAD support may have an improved survival on the transplant waiting list. ${ }^{599}$ Accordingly, MCS devices, particularly continuous-flow LVADs, are increasingly seen as an alternative to heart transplantation. Initially LVADs were developed for use as a short-term BTT approach (Table 13.1), ${ }^{600}$ but they are now being used for months to years in patients who will either face a long-term wait on the transplant list (currently only $10 \%$ of patients with an MCS device implanted with a BTT indication will receive an organ within 1 year of listing) or in patients who are not eligible for transplantation as permanent therapy or destination therapy. The number of patients with a permanent LVAD who are considered neither too old nor ineligible for transplantation is constantly growing. For a majority of these patients, lifelong LVAD therapy, despite eligibility for transplantation, has become a clinical reality. Current 2-3-year survival rates in carefully selected patients receiving the latest continuous flow devices are excellent, and comparable to early survival after heart transplantation. ${ }^{595}$ However, fewer data are available 
Table 13.2 INTERMACS (Interagency Registry for Mechanically Assisted Circulatory Support) stages for classifying patients with advanced heart failure

\begin{tabular}{|c|c|c|c|c|}
\hline INTERMACS level & $\begin{array}{l}\text { NYHA } \\
\text { Class }\end{array}$ & Description & Device & $\begin{array}{l}\text { ly survival with } \\
\text { LVAD therapy }\end{array}$ \\
\hline $\begin{array}{l}\text { I. Cardiogenic shock } \\
\text { "Crash and burn" }\end{array}$ & IV & $\begin{array}{l}\text { Haemodynamic instability in spite of increasing doses of catecholamines } \\
\text { and/or mechanical circulatory support with critical hypoperfusion of target } \\
\text { organs (severe cardiogenic shock). }\end{array}$ & $\begin{array}{l}\text { ECLS, ECMO, } \\
\text { percutaneous } \\
\text { support devices }\end{array}$ & $52.6 \pm 5.6 \%$ \\
\hline $\begin{array}{l}\text { 2. Progressive decline } \\
\text { despite inotropic } \\
\text { support "Sliding on } \\
\text { inotropes" }\end{array}$ & IV & $\begin{array}{l}\text { Intravenous inotropic support with acceptable blood pressure but rapid } \\
\text { deterioration of renal function, nutritional state, or signs of congestion. }\end{array}$ & $\begin{array}{l}\text { ECLS, ECMO, } \\
\text { LVAD }\end{array}$ & $63.1 \pm 3.1 \%$ \\
\hline $\begin{array}{l}\text { 3. Stable but inotrope } \\
\text { dependent "Dependent } \\
\text { stability" }\end{array}$ & IV & $\begin{array}{l}\text { Haemodynamic stability with low or intermediate doses of inotropics, but } \\
\text { necessary due to hypotension, worsening of symptoms, or progressive } \\
\text { renal failure. }\end{array}$ & LVAD & $78.4 \pm 2.5 \%$ \\
\hline $\begin{array}{l}\text { 4. Resting symptoms } \\
\text { "Frequent flyer" }\end{array}$ & $\begin{array}{l}\text { IV } \\
\text { ambulatory }\end{array}$ & $\begin{array}{l}\text { Temporary cessation of inotropic treatment is possible, but patient presents } \\
\text { with frequent symptom recurrences and typically with fluid overload. }\end{array}$ & LVAD & $78.7 \pm 3.0 \%$ \\
\hline $\begin{array}{l}\text { 6. Exertion limited } \\
\text { "Walking wounded" }\end{array}$ & III & $\begin{array}{l}\text { Minor limitation on physical activity and absence of congestion while at } \\
\text { rest. Easily fatigued by light activity. }\end{array}$ & $\begin{array}{l}\text { LVAD / Discuss } \\
\text { LVAD as option }\end{array}$ & - \\
\hline 7. "Placeholder" & III & Patient in NYHA Class III with no current or recent unstable fluid balance. & $\begin{array}{l}\text { Discuss LVAD } \\
\text { as option }\end{array}$ & - \\
\hline
\end{tabular}

ECLS = extracorporeal life support; ECMO = extracorporeal membrane oxygenation; INTERMACS = Interagency Registry for Mechanically Assisted Circulatory Support; LVAD = left ventricular assist device; NYHA = New York Heart Association.

${ }^{a}$ Kaplan-Meier estimates with standard error of the mean for 1 year survival with LVAD therapy. Patients were censored at time of last contact, recovery or heart transplantation. Due to small numbers outcomes for INTERMACS levels 5, 6, 7 were combined $^{610}$.

for longer-term outcomes. Among patients with continuous-flow LVADs, actuarial survival is $80 \%$ at 1 year and $70 \%$ at 2 years in predominantly non-transplant-eligible patients. Notably, survival of $85 \%$ at 2 years was recorded for patients up to 70 years of age without diabetes, renal impairment or cardiogenic shock. ${ }^{601,602}$ Patients receiving LVAD devices as BTT have a post-transplant survival rate similar or better than those not requiring or receiving bridging. ${ }^{599}$ Despite technological improvements, bleeding, thromboembolism (both of which can cause stroke), pump thrombosis, driveline infections and device failure remain significant problems and affect the long-term outcome of patients on MCS. ${ }^{599,603-606}$ It is recommended that such devices should only be implanted and managed at centres with appropriately trained specialist HF physicians and surgeons and an outpatient LVAD clinic with trained nursing staff. ${ }^{607}$

In some patients, LV reverse remodelling and functional improvement during MCS may permit removal of the LVAD ["bridge to recovery' (BTR)]. This outcome is more likely in younger patients with an acute fulminant but reversible cause of HF, such as acute myocarditis or peripartum cardiomyopathy. ${ }^{608,609}$ LVADs may also be used as a 'bridge to candidacy' (BTC) in order to permit recovery of end-organ dysfunction, improve RV function and relieve pulmonary hypertension, which may allow initially ineligible patients to become eligible for heart transplantation.

Earlier ventricular assist device (VAD) implantation in less severely ill patients, e.g. those not yet on inotropic support, was tested in a recent trial that revealed better outcomes than in those patients continuing on medical therapy. ${ }^{605}$ The INTERMACS registry likewise shows better outcomes in patients implanted with a higher
INTERMACS class, although the majority of VAD implants are done at INTERMACS levels $1-3 .^{604,610}$ Additionally, it needs to be remembered that no RCTs exist comparing medical therapy vs. MCS devices in these transplant-eligible patients (Table 13.2).

Typically, patients with end-stage HF considered for MCS exhibit many clinical hallmarks of declining cardiovascular function ${ }^{593}$ and may already be on continuous inotropic support or manifest a decline in end-organ function. Markers of liver and renal dysfunction, haematologic and coagulation abnormalities and lower serum albumin levels are associated with worse outcome. ${ }^{611,612}$

Evaluation of RV function is crucial since postoperative RV failure greatly increases perioperative mortality and reduces survival to, and after, transplantation. There are, however, multiple approaches to assessment of the RV (see Section 5.2.3). If RV failure is expected to be potentially reversible, temporary (days to weeks) extracorporeal right ventricular assist device (RVAD) support using a centrifugal pump in addition to LVAD implantation may be considered. For patients with chronic biventricular failure or a high risk for persisting RV failure after LVAD implantation, implantation of a biventricular assist device (BiVAD) may be necessary. Patients requiring long-term BiVAD support must be transplant-eligible, as BiVAD therapy is not suitable for destination therapy. The outcomes of $\mathrm{Bi}$ VAD therapy are inferior to those for LVAD therapy and therefore the indication for VAD therapy should be discussed before RV function deteriorates. The implantation of a total artificial heart with removal of the native heart should be restricted to selected patients who cannot be treated with an LVAD (unrepairable ventricular septal defect, cardiac rupture). 
Table I3.3 Patients potentially eligible for implantation of a left ventricular assist device

\begin{tabular}{|l|}
\hline $\begin{array}{l}\text { Patients with }>2 \text { months of severe symptoms despite optimal } \\
\text { medical and device therapy and more than one of the following: }\end{array}$ \\
\hline $\mathrm{LVEF}<25 \%$ and, if measured, peak $\mathrm{VO}_{2}<12 \mathrm{~mL} / \mathrm{kg} / \mathrm{min}$. \\
\hline$\geq 3 \mathrm{HF}$ hospitalizations in previous 12 months without an obvious \\
precipitating cause. \\
\hline Dependence on i.v. inotropic therapy. \\
\hline $\begin{array}{l}\text { Progressive end-organ dysfunction (worsening renal and/or hepatic } \\
\text { function) due to reduced perfusion and not to inadequate ventricular filling } \\
\text { pressure (PCWP } \geq 20 \text { mmHg and SBP } \leq 80-90 \mathrm{mmHg} \text { or } \mathrm{Cl} \leq 2 \mathrm{~L} / \mathrm{min} / \mathrm{m}^{2} \text { ). }\end{array}$ \\
\hline $\begin{array}{l}\text { Absence of severe right ventricular dysfunction together with severe } \\
\text { tricuspid regurgitation. }\end{array}$ \\
\hline
\end{tabular}

$\mathrm{Cl}=$ cardiac index; $\mathrm{HF}=$ heart failure; i.v. = intravenous; $\mathrm{LVEF}=$ left ventricular ejection fraction; $\mathrm{PCWP}=$ pulmonary capillary wedge pressure; $\mathrm{SBP}=$ systolic blood pressure; $\mathrm{VO}_{2}=$ oxygen consumption.

Patients with active infection, severe renal, pulmonary or hepatic dysfunction or uncertain neurological status after cardiac arrest or due to cardiogenic shock are not usually candidates for BTT or DT but may be candidates for BTC (Table 13.3).

\section{Recommendations for implantation of mechanical circulatory support in patients with refractory heart failure}

\begin{tabular}{|c|c|c|c|}
\hline Recommendations & Class $^{a}$ & Level $^{\mathrm{b}}$ & $\operatorname{Ref}^{c}$ \\
\hline $\begin{array}{l}\text { An LVAD should be considered in } \\
\text { patients who have end- stage HFrEF } \\
\text { despite optimal medical and device } \\
\text { therapy and who are eligible for } \\
\text { heart transplantation in order } \\
\text { to improve symptoms, reduce the } \\
\text { risk of HF hospitalization and the } \\
\text { risk of premature death (Bridge to } \\
\text { transplant indication). }\end{array}$ & Ila & C & \\
\hline $\begin{array}{l}\text { An LVAD should be considered in } \\
\text { patients who have end-stage HFrEF } \\
\text { despite optimal medical and device } \\
\text { therapy and who are not eligible for } \\
\text { heart transplantation to, reduce the } \\
\text { risk of premature death. }\end{array}$ & Ila & B & $\begin{array}{c}605,612 \\
613\end{array}$ \\
\hline
\end{tabular}

$\mathrm{HF}=$ heart failure; HFrEF = heart failure with reduced ejection fraction; LVAD = left ventricular assist device.

${ }^{\mathrm{a}}$ Class of recommendation.

bLevel of evidence.

${ }^{\mathrm{c}}$ Reference(s) supporting levels of evidence.
Table I 3.4 Heart transplantation: indications and contra-indications

\begin{tabular}{|l|l|}
\hline $\begin{array}{l}\text { Patients to } \\
\text { consider }\end{array}$ & $\begin{array}{l}\text { End-stage HF with severe symptoms, a poor prognosis, } \\
\text { and no remaining alternative treatment options. } \\
\text { Motivated, well informed, and emotionally stable. } \\
\text { Capable of complying with the intensive treatment } \\
\text { required postoperatively. }\end{array}$ \\
\hline indications & $\begin{array}{l}\text { Active infection. } \\
\text { Severe peripheral arterial or cerebrovascular disease. } \\
\text { Pharmacologically irreversible pulmonary hypertension } \\
\text { (LVAD should be considered with a subsequent re- } \\
\text { evaluation to establish candidacy). } \\
\text { Cancer (a collaboration with oncology specialists } \\
\text { should occur to stratify each patient as to their risk of } \\
\text { tumour recurrence). } \\
\text { Irreversible renal dysfunction (e.g. creatinine clearance } \\
<30 \mathrm{~mL} / \text { min). } \\
\text { Systemic disease with multi-organ involvement. } \\
\text { Other serious co-morbidity with poor prognosis. } \\
\text { Pre-transplant BMI }>35 \mathrm{~kg} / \mathrm{m}^{2} \text { (weight loss is } \\
\text { recommended to achieve a BMI <35 kg/m²). } \\
\text { Current alcohol or drug abuse. } \\
\text { Any patient for whom social supports are deemed } \\
\text { insufficient to achieve compliant care in the outpatient } \\
\text { setting. }\end{array}$ \\
\hline
\end{tabular}

$\mathrm{BMI}=$ body mass index; $\mathrm{HF}=$ heart failure; $\mathrm{LVAD}=$ left ventricular assist device

\subsection{Heart transplantation}

Heart transplantation is an accepted treatment for end-stage HF. ${ }^{614,615}$ Although controlled trials have never been conducted, there is a consensus that transplantation-provided that proper selection criteria are applied — significantly increases survival, exercise capacity, quality of life and return to work compared with conventional treatment.

Apart from the shortage of donor hearts, the main challenges in transplantation are the consequences of the limited effectiveness and complications of immunosuppressive therapy in the long term (i.e. antibody-mediated rejection, infection, hypertension, renal failure, malignancy and coronary artery vasculopathy). The indications for and contraindications to heart transplantation have recently been updated and are summarized in Table $13.4 .{ }^{616} \mathrm{It}$ needs to be considered that some contraindications are transient and treatable. While an active infection remains a relative contraindication to heart transplantation, patients with HIV, hepatitis, Chagas disease and tuberculosis can be considered as suitable candidates provided certain strict management principles are adhered to by the teams. In patients with cancer requiring heart transplantation, a close collaboration with oncology specialists should occur to stratify each patient as to their risk of tumour recurrence. ${ }^{616}$ 


\section{Table I4.I Characteristics and components of management programmes for patients with heart failure}

\begin{tabular}{|c|c|}
\hline \multirow[t]{3}{*}{ Characteristics } & $\begin{array}{l}\text { Should employ a multidisciplinary approach } \\
\text { (cardiologists, primary care physicians, nurses, } \\
\text { pharmacists, physiotherapists, dieticians, social } \\
\text { workers, surgeons, psychologists, etc.). }\end{array}$ \\
\hline & Should target high-risk symptomatic patients. \\
\hline & $\begin{array}{l}\text { Should include competent and professionally } \\
\text { educated staff. }{ }^{617}\end{array}$ \\
\hline \multirow[t]{9}{*}{ Components } & Optimized medical and device management. \\
\hline & $\begin{array}{l}\text { Adequate patient education, with special emphasis } \\
\text { on adherence and self-care. }\end{array}$ \\
\hline & $\begin{array}{l}\text { Patient involvement in symptom monitoring and } \\
\text { flexible diuretic use. }\end{array}$ \\
\hline & $\begin{array}{l}\text { Follow-up after discharge (regular clinic and/or } \\
\text { home-based visits; possibly telephone support or } \\
\text { remote monitoring). }\end{array}$ \\
\hline & $\begin{array}{l}\text { Increased access to healthcare (through in-person } \\
\text { follow-up and by telephone contact; possibly } \\
\text { through remote monitoring). }\end{array}$ \\
\hline & $\begin{array}{l}\text { Facilitated access to care during episodes of } \\
\text { decompensation. }\end{array}$ \\
\hline & $\begin{array}{l}\text { Assessment of (and appropriate intervention in } \\
\text { response to) an unexplained change in weight, } \\
\text { nutritional status, functional status, quality of life, } \\
\text { or laboratory findings. }\end{array}$ \\
\hline & Access to advanced treatment options. \\
\hline & $\begin{array}{l}\text { Provision of psychosocial support to patients and } \\
\text { family and/or caregivers. }\end{array}$ \\
\hline
\end{tabular}

The use of mechanical circulatory support, particularly LVAD, should be considered for patients with potentially reversible or treatable co-morbidities, such as cancer, obesity, renal failure, tobacco use and pharmacologically irreversible pulmonary hypertension, with a subsequent re-evaluation to establish candidacy.

\section{Multidisciplinary team management}

Non-pharmacological non-device/surgical interventions used in the management of HF (both HFrEF and HFpEF) are summarized in Tables 14.1 and 14.2 and detailed practical recommendations on their use have been published by the HFA of the ESC. ${ }^{591,592}$ There is no evidence that these on their own improve mortality, morbidity or quality of life. For this reason, these interventions have not been given a recommendation with an evidence level. The exceptions are implementation of care in a multidisciplinary framework, monitoring and exercise training (see recommendations table), all of which are discussed below.
Recommendations for exercise, multidisciplinary management and monitoring of patients with heart failure

\begin{tabular}{|c|c|c|c|}
\hline Recommendations & Class $^{a}$ & Level $^{b}$ & $\operatorname{Ref}^{c}$ \\
\hline $\begin{array}{l}\text { It is recommended that regular } \\
\text { aerobic exercise is encouraged } \\
\text { in patients with HF to improve } \\
\text { functional capacity and symptoms. }\end{array}$ & $\mathbf{I}$ & $\mathbf{A}$ & $\begin{array}{c}321 \\
618-621\end{array}$ \\
\hline $\begin{array}{l}\text { It is recommended that regular } \\
\text { aerobic exercise is encouraged in } \\
\text { stable patients with HFrEF to reduce } \\
\text { the risk of HF hospitalization. }\end{array}$ & $\mathbf{I}$ & $\mathbf{A}$ & 618,619 \\
\hline $\begin{array}{l}\text { It is recommended that patients with } \\
\mathrm{HF} \text { are enrolled in a multidisciplinary } \\
\text { care management programme to } \\
\text { reduce the risk of HF hospitalization } \\
\text { and mortality. }\end{array}$ & I & $\mathbf{A}$ & $622-625$ \\
\hline $\begin{array}{l}\text { Referral to primary care for long- } \\
\text { term follow-up may be considered } \\
\text { for stable HF patients who are on } \\
\text { optimal therapy to monitor for } \\
\text { effectiveness of treatment, disease } \\
\text { progression and patient adherence. }\end{array}$ & Ilb & B & 626,627 \\
\hline $\begin{array}{l}\text { Monitoring of pulmonary artery } \\
\text { pressures using a wireless } \\
\text { implantable haemodynamic } \\
\text { monitoring system (CardioMems) } \\
\text { may be considered in symptomatic } \\
\text { patients with HF with previous } \\
\text { HF hospitalization in order to } \\
\text { reduce the risk of recurrent HF } \\
\text { hospitalization. }\end{array}$ & Illb & B & 628,629 \\
\hline $\begin{array}{l}\text { Multiparameter monitoring based } \\
\text { on ICD (IN-TIME approach) may be } \\
\text { considered in symptomatic patients } \\
\text { with HFrEF (LVEF } \leq 35 \% \text { ) in order to } \\
\text { improve clinical outcomes. }\end{array}$ & Ilb & B & 630 \\
\hline
\end{tabular}

$\mathrm{HF}=$ heart failure; $\mathrm{HFrEF}=$ heart failure with reduced ejection fraction; ICD = implantable cardioverter-defibrillator; LVEF = left ventricular ejection fraction, IN-TIME = Implant-based multiparameter telemonitoring of patients with heart failure. ${ }^{a}$ Class of recommendation.

bLevel of evidence.

${ }^{\mathrm{c}}$ Reference(s) supporting levels of evidence.

\subsection{Organization of care}

The goal of management of HF is to provide a 'seamless' system of care that embraces both the community and hospital throughout the health care journey. The standards of care that patients with HF should expect have been published by the ESC HFA. ${ }^{591}$ To achieve this goal, other services, such as cardiac rehabilitation and palliative care, must be integrated into the overall provision for patients with HF. Fundamental to the delivery of this complete package of care are multidisciplinary management programmes designed to improve outcomes through structured follow-up with patient education, optimization of medical treatment, psychosocial support and improved access to care (Table 14.1). Such strategies reduce HF hospitalization and mortality in patients discharged from the hospital. ${ }^{624,625}$

Key to the success of these programmes is coordination of care along the continuum of HF and throughout the chain of care 
Table 14.2 Key topics and self-care skills to include in patient education and the professional behaviours to optimize learning and facilitate shared decision making

\begin{tabular}{|c|c|c|}
\hline Education topic & Patient skills & Professional behaviours \\
\hline $\begin{array}{l}\text { Definition, } \\
\text { aetiology and } \\
\text { trajectory of } \\
\text { HF (including } \\
\text { prognosis). }\end{array}$ & $\begin{array}{l}\text { - Understand the cause of HF, symptoms and disease } \\
\text { trajectory. } \\
\text { - Make realistic decisions including decisions about } \\
\text { treatment at end-of-life. }\end{array}$ & $\begin{array}{l}\text { - Provide oral and written information that takes account of educational } \\
\text { grade and health literacy. } \\
\text { - Recognize HF disease barriers to communication and provide } \\
\text { information at regular time intervals. } \\
\text { - Sensitively communicate information on prognosis at time of diagnosis, } \\
\text { during decision making about treatment options, when there is a } \\
\text { change in the clinical condition and whenever the patient requests. }\end{array}$ \\
\hline $\begin{array}{l}\text { Symptom } \\
\text { monitoring and } \\
\text { self-care. }\end{array}$ & $\begin{array}{l}\text { - Monitor and recognize change in signs and symptoms. } \\
\text { - Know how and when to contact a healthcare professional. } \\
\text { - In line with professional advice, know when to self- } \\
\text { manage diuretic therapy and fluid intake. }\end{array}$ & $\begin{array}{l}\text { - Provide individualized information to support self-management such as: } \\
\Rightarrow \text { In the case of increasing dyspnoea or oedema or a sudden } \\
\text { unexpected weight gain of }>2 \mathrm{~kg} \text { in } 3 \text { days, patients may increase } \\
\text { their diuretic dose and/or alert their healthcare team. } \\
\Rightarrow \text { Use of flexible diuretic regime. } \\
\Rightarrow \text { Self-care support aids such as dosette box when appropriate. }\end{array}$ \\
\hline $\begin{array}{l}\text { Pharmacological } \\
\text { treatment. }\end{array}$ & $\begin{array}{l}\text { - Understand the indications, dosing and side effects of drugs. } \\
\text { - Recognize the common side effects and know } \\
\text { when to notify a healthcare professional. } \\
\text { - Recognize the benefits of taking medication as prescribed. }\end{array}$ & $\begin{array}{l}\text { Provide written and oral information on dosing, effects and side } \\
\text { effects (see web tables 7.4-7.8 - practical guidance on use of } \\
\text { pharmacological agents). }\end{array}$ \\
\hline $\begin{array}{l}\text { Implanted devices } \\
\text { and percutaneous/ } \\
\text { surgical } \\
\text { interventions. }\end{array}$ & $\begin{array}{l}\text { - Understand the indications and aims of procedures/ } \\
\text { implanted devices. } \\
\text { - Recognize the common complications and know when to } \\
\text { notify a healthcare professional. } \\
\text { - Recognize the importance and benefits of procedures/ } \\
\text { implanted devices. }\end{array}$ & $\begin{array}{l}\text { - Provide written and oral information on benefits and side effects. } \\
\text { - Provide written and oral information on regular control of device } \\
\text { functioning, along with documentation of regular check-up. }\end{array}$ \\
\hline Immunization & $\begin{array}{l}\text { - Receive immunization against influenza and pneumococcal } \\
\text { disease }\end{array}$ & - Advise on local guidance and immunization practice. \\
\hline Diet and alcohol & $\begin{array}{l}\text { - Avoid excessive fluid intake. } \\
\text { - Recognize need for altered fluid intake such as: } \\
\Rightarrow \quad \text { Increase intake during periods of high heat and } \\
\text { humidity, nausea/vomiting } \\
\Rightarrow \quad \text { Fluid restriction of } 1.5-2 \mathrm{~L} / \text { day may be considered in } \\
\text { patients with severe HF to relieve symptoms and } \\
\text { congestion. } \\
\text { - Monitor body weight and prevent malnutrition. } \\
\text { - Eat healthily, avoid excessive salt intake ( }>6 \mathrm{~g} / \text { day) and } \\
\text { maintain a healthy body weight. } \\
\text { - Abstain from or avoid excessive alcohol intake, especially } \\
\text { for alcohol induced cardiomyopathy. }\end{array}$ & $\begin{array}{l}\text { - Individualize information on fluid intake to take into account body } \\
\text { weight and periods of high heat and humidity.Adjust advice during } \\
\text { periods of acute decompensation and consider altering these } \\
\text { restrictions towards end-of-life. } \\
\text { - Tailor alcohol advice to aetiology of HF; e.g. abstinence in } \\
\text { alcoholic cardiomyopathy. } \\
\text { - Normal alcohol guidelines apply ( } 2 \text { units per day in men or I unit per } \\
\text { day in women). I unit is } 10 \mathrm{~mL} \text { of pure alcohol (e.g. I glass of wine, I/2 } \\
\text { pint of beer, I measure of spirit). } \\
\text { - For management of obesity (see Section II.I5). }\end{array}$ \\
\hline $\begin{array}{l}\text { Smoking and } \\
\text { recreational } \\
\text { substance use. }\end{array}$ & - Stop smoking and taking recreational substances. & $\begin{array}{l}\text { - Refer for specialist advice for smoking cessation and drug withdrawal } \\
\text { and replacement therapy. } \\
\text { - Consider referral for cognitive behavioural theory and psychological } \\
\text { support if patient wishes support to stop smoking. }\end{array}$ \\
\hline Exercise & $\begin{array}{l}\text { - Undertake regular exercise sufficient to provoke mild or } \\
\text { moderate breathlessness. }\end{array}$ & $\begin{array}{l}\text { - Advice on exercise that recognizes physical and functional limitations, } \\
\text { such as frailty, comorbidities. } \\
\text { - Referral to exercise programme when appropriate. }\end{array}$ \\
\hline Travel and leisure & $\begin{array}{l}\text { - Prepare travel and leisure activities according to physical } \\
\text { capacity. } \\
\text { - Monitor and adapt fluid intake according to humidity } \\
\text { (flights and humid climates). } \\
\text { - Be aware of adverse reactions to sun exposure with } \\
\text { certain medication (such as amiodarone). } \\
\text { - Consider effect of high altitude on oxygenation. } \\
\text { - take medicine in cabin luggage in the plane, have a list with } \\
\text { you of treatments and the dosage with the generic name. }\end{array}$ & $\begin{array}{l}\text { - Refer to local country specific driving regulations regarding ICD. } \\
\text { - Provide advice regarding flight security devices in presence of ICD. }\end{array}$ \\
\hline $\begin{array}{l}\text { Sleep and } \\
\text { breathing (see } \\
\text { co-morbidities } \\
\text { Section II.16). }\end{array}$ & $\begin{array}{l}\text { - Recognize problems with sleeping, their relationship with } \\
\mathrm{HF} \text { and how to optimize sleep. }\end{array}$ & $\begin{array}{l}\text { - Provide advice such as timing of diuretics, environment for sleep, device } \\
\text { support. } \\
\text { - In presence of sleep-disordered breathing provide advice on weight } \\
\text { reduction/control. }\end{array}$ \\
\hline $\begin{array}{l}\text { Sexual activity (see } \\
\text { co-morbidities } \\
\text { Section } 11.7) \text {. }\end{array}$ & $\begin{array}{l}\text { - Be reassured about engaging in sex, provided sexual } \\
\text { activity does not provoke undue symptoms. } \\
\text { - Recognize problems with sexual activity, their relationship } \\
\text { with HF and applied treatment and how to treat erectile } \\
\text { dysfunction. }\end{array}$ & $\begin{array}{l}\text { Provide advice on eliminating factors predisposing to erectile } \\
\text { dysfunction and available pharmacological treatment of erectile } \\
\text { dysfunction. } \\
\text { - Refer to specialist for sexual counselling when necessary. }\end{array}$ \\
\hline
\end{tabular}


Table 14.2 Key topics and self-care skills to include in patient education and the professional behaviours to optimize learning and facilitate shared decision making (continued)

\begin{tabular}{|c|c|c|}
\hline Education topic & Patient skills & Professional behaviours \\
\hline $\begin{array}{l}\text { Psychosocial } \\
\text { aspects }\end{array}$ & $\begin{array}{l}\text { - Understand that depressive symptoms and cognitive } \\
\text { dysfunction are found more frequently in people with HF, } \\
\text { and that they may affect adherence. } \\
\text { - Recognize psychological problems which may occur } \\
\text { in the course of disease, in relation to changed lifestyle, } \\
\text { pharmacotherapy, implanted devices and other } \\
\text { procedures (including mechanical support and heart } \\
\text { transplantation). }\end{array}$ & $\begin{array}{l}\text { - Regularly communicate information on disease, treatment options and } \\
\text { self-management. } \\
\text { - Involve family and carers in HF management and self-care. } \\
\text { - Refer to specialist for psychological support when necessary. }\end{array}$ \\
\hline
\end{tabular}

$\mathrm{HF}=$ heart failure; ICD = implantable cardioverter defibrillator.

delivered by the various services within the health care system. This necessitates close collaboration between HF practitioners (primarily cardiologists, HF nurses and general practitioners) and other experts, including pharmacists, dieticians, physiotherapists, psychologists, palliative care providers and social workers. The content and structure of HF management programmes may vary in different countries and health care settings. The components shown in Table 14.1 are recommended. HF services should be easily accessible to the patient and his/her family and care providers. A telephone helpline may facilitate access to professional advice.

The website http://ww.heartfailurematters.org is an option for professional information for those patients and families with Internet access.

\subsection{Discharge planning}

Early readmission after hospital discharge is common and may be addressed through coordinated discharge planning. The standards of care that patients should expect have been published by the HFA and the Acute Cardiac Care Association. ${ }^{540,631}$ Discharge planning should commence as soon as the patient's condition is stable. During hospital admission, providing patients with information and education for self-care improves outcome. Discharge should be arranged for when the patient is euvolaemic and any precipitants of the admission have been treated. Hospitals with early physician follow-up after discharge show reduced 30-day readmission, and those that initiated programmes to discharge patients with an outpatient follow-up appointment already scheduled experienced a greater reduction in readmissions than those not taking up this strategy. ${ }^{632}$

\subsection{Lifestyle advice}

There is little evidence that specific lifestyle advice improves quality of life or prognosis; however, providing this information has become a key component of education for self-care. Patients should be provided with sufficient up-to-date information to make decisions on lifestyle adjustment and self-care. Ideally for those patients admitted to the hospital, lifestyle advice should begin prior to discharge. Information should be individually tailored to need and take into account relevant co-morbidities that may influence retention of information (such as cognitive impairment and depression). Practical recommendations have been published by the HFA. ${ }^{591}$ Key topics to include are recommended in Table 14.2 .

\subsection{Exercise training}

Several systematic reviews and meta-analyses of small studies have shown that physical conditioning by exercise training improves exercise tolerance, health-related quality of life and HF hospitalization rates in patients with HF. A single large $\mathrm{RCT}^{618}$ showed a modest and non-significant reduction in the primary composite outcome of all-cause mortality or all-cause hospitalization. There was no reduction in mortality and no safety concerns were raised. ${ }^{618,633}$ The most recent Cochrane review of exercise training ${ }^{619}$ included 33 trials with 4740 patients with HF (predominantly HFrEF). There was a trend towards a reduction in mortality with exercise in trials with $>1$ year of follow-up. Compared with the control group, exercise training reduced the rate of overall and HF-specific hospitalization and improved quality of life. Practical recommendations on exercise training have been published by the HFA. ${ }^{120}$

There is evidence that in patients with HFpEF, exercise training has several benefits, including improvements in exercise capacity, as measured objectively using peak oxygen consumption, quality of life and diastolic function, assessed by echocardiography. ${ }^{321,620,621,634}$

Patients with HF, regardless of LVEF, are recommended to perform properly designed exercise training (see the recommendations table).

\subsection{Follow-up and monitoring}

Patients with HF benefit from regular follow-up and monitoring of biomedical parameters to ensure the safety and optimal dosing of medicines and detect the development of complications or disease progression that may require a change in management (e.g. the onset of AF or development of anaemia). Monitoring may be undertaken by the patients themselves during home visits, in community or hospital clinics, by remote monitoring with or without implanted devices or by structured telephone support (STS). The optimal method of monitoring will depend on local organizations and resources and will vary among patients. For example, more frequent monitoring will be required during periods of instability or optimization of medication. Older adults may also benefit from more frequent monitoring. Some patients will be keen and able to participate in self-monitoring.

High circulating NPs predict unfavourable outcomes in patients with $\mathrm{HF}$, and a decrease in NP levels during recovery from circulatory decompensation is associated with a better prognosis. ${ }^{588-590}$ Although it is plausible to monitor clinical status and tailor treatment based on changes in circulating NPs in patients with HF, published studies have provided differing results. ${ }^{635-638}$ This does not enable us to recommend a broad application of such an approach.

Telemedicine in HF, which is also termed remote patient management, has variable clinical trial results. ${ }^{639}$ Several meta-analyses suggest clinical benefits, but numerous prospectively initiated clinical trials including $>3700$ patients have not confirmed this. These clinical trials include Tele-HF, ${ }^{640}$ TIM-HF, ${ }^{641} \mathrm{INH}^{642}{ }^{642} \mathrm{WISH}^{643}$ and 
TEHAF. ${ }^{644}$ It is clear that there is not just one type of telemedicine, and each approach needs to be assessed on its individual merit.

Recently, two individual approaches were shown to be successful in improving clinical outcome when used in patients with HFpEF or HFrEF. These approaches include the CardioMems system (tested in 550 patients with both HFrEF and HFpEF) ${ }^{628}$ and the IN-TIME approach (tested in $664 \mathrm{HFrEF}$ patients) ${ }^{630}$, which may be considered for use in selected patients with HF (see the recommendations table).

\subsection{The older adult, frailty and cognitive impairment}

HF management and self-care behaviour are complicated by ageing, co-morbid conditions, cognitive impairment, frailty and limited social support. HF is also a leading cause of hospital admission in the older adult, where it is associated with increased hospital length of stay and risk of mortality. ${ }^{645}$

Frailty is common in older adults with $\mathrm{HF}$, with a recent study suggesting it may be present in $>70 \%$ of patients with $\mathrm{HF}$ and $>80$ years of age. ${ }^{645}$ Frailty scoring systems provide an objective assessment and identify the presence of or change in the level of frailty. Patients with a high frailty score will benefit from closer contact with the HF specialist team, more frequent follow-up and monitoring and individualized self-care support.

Frailty scores include ${ }^{646}$ walking speed (gait speed test), timed up-and-go test, PRISMA 7 questionnaire, Frail Score, ${ }^{647}$ Fried Score ${ }^{647,648}$ and Short Physical Performance Battery (SPPB).

Cognitive impairment and HF frequently coexist. Acute delirium is also associated with decompensated HF and may be present on hospital admission. Cognitive function can be assessed using the Mini-Mental State Examination ${ }^{649}$ or the Montreal cognitive assessment. ${ }^{650}$ The presence of delirium and HF is found more commonly in older adults and is associated with increased mortality and poorer self-care ability and increases any hospital length of stay. ${ }^{651}$ There is currently no clinical evidence that HF medication worsens or improves cognitive function. However, its effect on HF outcome suggests such medication should be used. Support from a multidisciplinary HF team in collaboration with specialist dementia support teams, alongside medication compliance aids, tailored self-care advice and involvement of family and caregivers, may improve adherence with complex HF medication and self-care regimens (see Table 14.2 on patient education) (Table 14.3).

\subsection{Palliative and end-of-life care}

Palliative care approaches include a focus on symptom management, emotional support and communication between the patient and his/ her family. Ideally this should be introduced early in the disease trajectory and increased as the disease progresses. A decision to alter the focus of care from modifying disease progression to optimising quality of life should be made in discussion with the patient, cardiologist, nurse and general practitioner. The patient's family should be involved in such discussions if requested by the patient ${ }^{652,653}$ (Table 14.4).

Key components of a palliative care service are recommended in Table 14.5. Palliative care has been discussed in detail in a position paper from the ESC HFA. ${ }^{654}$

Liaison between the specialist palliative care services and the HF team and/or the primary care physician, using a shared care
Table I4.3 Specific recommendations regarding monitoring and follow-up of the older adult with heart failure

Monitor frailty and seek and address reversible causes (cardiovascular and non-cardiovascular) of deterioration in frailty score.

Medication review: optimize doses of heart failure medication slowly and with frequent monitoring of clinical status. Reduce polypharmacy; number, doses and complexity of regime. Consider stopping medication without an immediate effect on symptom relief or quality of life (such as statin). Review the timing and dose of diuretic therapy to reduce risk of incontinence.

Consider need to refer to specialist care of the elderly team and to general practitioner and social worker, etc. for follow-up and support for the patient and his/her family.

Table I4.4 Patients with heart failure in whom end of life care should be considered

\begin{tabular}{l}
\hline $\begin{array}{l}\text { Progressive functional decline (physical and mental) and dependence in } \\
\text { most activities of daily living. }\end{array}$ \\
\hline $\begin{array}{l}\text { Severe heart failure symptoms with poor quality of life despite optimal } \\
\text { pharmacological and non-pharmacological therapies. }\end{array}$ \\
\hline $\begin{array}{l}\text { Frequent admissions to hospital or other serious episodes of } \\
\text { decompensation despite optimal treatment. }\end{array}$ \\
\hline Heart transplantation and mechanical circulatory support ruled out. \\
\hline Cardiac cachexia. \\
\hline Clinically judged to be close to end of life. \\
\hline
\end{tabular}

Table I4.5 Key components of palliative care service in patients with heart failure

Focus on improving or maintaining the quality of life of a patient and his/ her family as well as possible until he/she dies.

Frequent assessment of symptoms (including dyspnoea and pain) resulting from advanced heart failure and other co-morbidities and focus on symptom relief.

Access for the patient and his/her family to psychological support and spiritual care according to need.

Advanced care planning, taking account of preferences for place of death and resuscitation (which may include deactivating devices, such as pacemaker and/or implantable cardioverter defibrillator).

approach, is required in order to address and optimally coordinate the patient's care. Recent pilot studies have suggested an improvement in symptom burden and quality of life, ${ }^{653,655}$ but these data are too limited to provide a recommendation.

Specific therapies and actions may provide palliation of symptoms and improve quality of life but have a limited evidence base:

- Morphine (with an antiemetic when high doses are needed) can be used to reduce breathlessness, pain and anxiety. ${ }^{656}$ 
- Increasing the inspired oxygen concentration may provide relief of dyspnoea.

- Diuretic management can be used to relieve severe congestion or optimize symptom control (congestion and thirst).

- Reduce HF drugs that reduce blood pressure to maintain sufficient oxygenation and reduce the risk of falls.

Ideally these therapies should be delivered in the patient's home. In the majority of cases the whole family should receive social support. ${ }^{652}$

A management plan should be developed through discussion with the patient and family. It should include

- A discussion about stopping medication that does not have an immediate effect on symptom management or health-related quality of life, such as agents to lower cholesterol or treat osteoporosis

- Documentation of the patient's decision regarding resuscitation attempts

- Deactivation of an ICD at end-of-life (according to local legal regulations)

- Preferred place for care and death

- Emotional support to the patient and family/caregiver with appropriate referral for psychological or spiritual support

Clearly, symptoms and quality of life change over time and regular reassessment is recommended. Palliative scores provide an objective assessment of the patient's symptoms and needs and may help establish the effectiveness of therapy.

Palliative outcome scores include the Palliative Care Outcome Scale, ${ }^{657}$ Karnofsky Performance Status ${ }^{658}$ and Functional Assessment of Chronic Illness Therapy - Palliative Care (FACIT-Pal). ${ }^{659}$

\section{Gaps in evidence}

Clinicians responsible for managing patients with HF must frequently make treatment decisions without adequate evidence or a consensus of expert opinion. The following is a short list of selected, common issues that deserve to be addressed in future clinical research.

1. Definition, diagnosis, epidemiology

- For HFmrEF/HFpEF, research into the underlying characteristics, pathophysiology and diagnosis (with new modalities)

- Updated epidemiology on HF incidence and prevalence including patients from all continents

- For imaging and biomarkers, studies on effects of specific imaging modalities and biomarkers to improve clinical outcome (e.g. biomarker-guided therapies, detection of CAD/myocardial ischaemia, late-gadolinium enhancement CMR, echocardiographic strain measurements, stress echocardiography, etc.)

- Increasing awareness of HF in the medical community, lay public and among policy makers.

2. Strategies aimed at prevention and screening of HF

- Evaluate the comparative clinical effectiveness and costeffectiveness of different strategies to screen for HF.

3. Pharmacological therapy

- Identification of non-responders to current guideline-advised medical treatment
- Targeted therapies for specific aetiologies of HFrEF (e.g. myocarditis, peripartum cardiomyopathy)

- Therapies directly improving cardiomyocyte function (e.g. acto-myosin cross-bridge activation, sarco/endoplasmic reticulum $\mathrm{Ca}^{2+}$-ATPase activation, ryanodine receptor stabilization, energetic modulation) or targeting non-myocytic compartment (e.g. anti-fibrosis/matrix remodelling)

- Therapies for HFmrEF/HFpEF (ARNIs, beta-blockers, soluble guanyl cyclase inhibitors, i.v. iron)

4. Devices and interventions

- Indications for ICDs in specific subgroups (e.g. ARVC and HFmrEF/HFpEF) and optimal selection of ICD candidates

- QRS morphology or duration as a predictor of response to CRT

- CRT in patients with AF

- Efficacy of PV ablation as a rhythm-control strategy in patients with $\mathrm{AF}$

- Interventional approach to recurrent, life-threatening ventricular tachyarrhythmias

- The role of remote monitoring strategies in HF

- Non-surgical (percutaneous) correction of functional mitral and tricuspid regurgitations

- Identification of indications for coronary angiography/revascularization in patients with HF and chronic stable CAD

- Effects of novel LVADs as destination therapy and bridge to transplantation

5. Co-morbidities

- A better understanding of pathophysiology and potential treatments in specific HF populations, including the

- very elderly,

- young patients,

- eGFR $<30 \mathrm{~mL} / \mathrm{min}$,

- diabetic patients,

- cardiotoxic chemotherapy-induced HF,

- muscular dystrophies,

- cachexia and depression.

- Therapies for HF-related sleep-disordered breathing in HFrEF/ HFpEF/HFmrEF.

6. Acute heart failure

- Prospective evaluation of the 'time-to-treatment' concept in AHF

- Evaluation of whether inadequate phenotyping is responsible for the failure of treatments to improve outcome in AHF

- Better definition and treatment of diuretic resistance

- Role of nitrates in the management of AHF

- Treatments improving mortality and morbidity

- Strategies and therapies to prevent early rehospitalization after discharge for a hospital admission for AHF.

7. Other/remaining aspects

- Treatment algorithms for patients with HF excluded by pivotal clinical trials

- Palliative and end-of-life care management and assessment of outcome

- Optimal integration of multidisciplinary care, self-management of patients and their adherence. 


\section{To do and not to do messages from the Guidelines}

\begin{tabular}{|c|c|c|}
\hline Recommendations for cardiac imaging in patients with suspected or established heart failure & Class $^{a}$ & Level $^{\mathrm{b}}$ \\
\hline $\begin{array}{l}\text { TTE is recommended for the assessment of myocardial structure and function in subjects with suspected HF in order to establish a } \\
\text { diagnosis of either HFrEF, HFmrEF or HFpEF. }\end{array}$ & I & C \\
\hline $\begin{array}{l}\text { TTE is recommended for the assessment of LVEF in order to identify patients with HF who would be suitable for evidence-based } \\
\text { pharmacological and device (ICD, CRT) treatment recommended for HFrEF. }\end{array}$ & I & C \\
\hline $\begin{array}{l}\text { Recommendations aiming to prevent or delay the development of overt heart failure or prevent death } \\
\text { before the onset of symptoms }\end{array}$ & Class $^{a}$ & Level $^{\mathrm{b}}$ \\
\hline Treatment of hypertension is recommended to prevent or delay the onset of $\mathrm{HF}$ and prolong life. & I & $\mathbf{A}$ \\
\hline $\begin{array}{l}\text { ACE-I is recommended in patients with asymptomatic LV systolic dysfunction and a history of myocardial infarction in order to } \\
\text { prevent or delay the onset of HF and prolong life. }\end{array}$ & I & A \\
\hline $\begin{array}{l}\text { Beta-blocker is recommended in patients with asymptomatic LV systolic dysfunction and a history of myocardial infarction, in order } \\
\text { to prevent or delay the onset of HF and prolong life. }\end{array}$ & I & B \\
\hline $\begin{array}{l}\text { Pharmacological treatments indicated in patients with symptomatic heart failure with reduced ejection } \\
\text { fraction }\end{array}$ & Class $^{a}$ & Level $^{\mathrm{b}}$ \\
\hline $\begin{array}{l}\text { An ACE-Ic is recommended, in addition to a beta-blocker, for symptomatic patients with } \mathrm{HFrEF} \text { to reduce the risk of HF } \\
\text { hospitalization and death. }\end{array}$ & I & A \\
\hline $\begin{array}{l}\text { A beta-blocker is recommended, in addition to an ACE- } \mathrm{l}^{\mathrm{c}} \text {, for patients with stable, symptomatic HFrEF to reduce the risk of HF } \\
\text { hospitalization and death. }\end{array}$ & $\mathbf{I}$ & $\mathbf{A}$ \\
\hline $\begin{array}{l}\text { An MRA is recommended for patients with HFrEF, who remain symptomatic despite treatment with an ACE-Ic and a beta-blocker, to } \\
\text { reduce the risk of HF hospitalization and death. }\end{array}$ & $\mathbf{I}$ & $\mathbf{A}$ \\
\hline $\begin{array}{l}\text { Other pharmacological treatments recommended in selected patients with symptomatic heart failure with } \\
\text { reduced ejection fraction }\end{array}$ & Class $^{a}$ & Level $^{\mathrm{b}}$ \\
\hline Diuretics are recommended in order to improve symptoms and exercise capacity in patients with signs and/or symptoms of congestion. & I & B \\
\hline $\begin{array}{l}\text { Sacubitril/valsartan is recommended as a replacement for an ACE-I to further reduce the risk of HF hospitalization and death in } \\
\text { ambulatory patients with HFrEF who remain symptomatic despite optimal treatment with an ACE-I, a beta-blocker and an MRA. }\end{array}$ & I & B \\
\hline $\begin{array}{l}\text { Treatments (or combinations of treatments) that may cause harm in patients with symptomatic (New York } \\
\text { Heart Association Class II-IV) heart failure with reduced ejection fraction }\end{array}$ & Class $^{a}$ & Level $^{\mathrm{b}}$ \\
\hline Diltiazem or verapamil are not recommended in patients with $\mathrm{HFrEF}$, as they increase the risk of $\mathrm{HF}$ worsening and $\mathrm{HF}$ hospitalization. & III & C \\
\hline $\begin{array}{l}\text { The addition of an ARB (or a renin inhibitor) to the combination of an ACE-I and an MRA is not recommended in patients with HF, } \\
\text { because of the increased risk of renal dysfunction and hyperkalaemia. }\end{array}$ & III & C \\
\hline Recommendations for implantable cardioverter-defibrillator in patients with heart failure & Class $^{a}$ & Level $^{\mathrm{b}}$ \\
\hline $\begin{array}{l}\text { Secondary prevention } \\
\text { An ICD is recommended to reduce the risk of sudden death and all-cause mortality in patients who have recovered from a ventricular } \\
\text { arrhythmia causing haemodynamic instability, and who are expected to survive for }>I \text { year with good functional status. }\end{array}$ & $\mathbf{I}$ & $\mathbf{A}$ \\
\hline $\begin{array}{l}\text { Primary prevention } \\
\text { An ICD is recommended to reduce the risk of sudden death and all-cause mortality in patients with symptomatic HF (NYHA Class } \\
\text { II-III), and an LVEF } \leq 35 \% \text { despite } \geq 3 \text { months of OMT, provided they are expected to survive substantially longer than I year with good } \\
\text { functional status, and they have: } \\
\text { - IHD (unless they have had an MI in the prior } 40 \text { days) } \\
\text { - DCM }\end{array}$ & $\begin{array}{l}\text { I } \\
\text { I }\end{array}$ & $\begin{array}{l}\text { A } \\
\text { B }\end{array}$ \\
\hline ICD implantation is not recommended within 40 days of an $\mathrm{MI}$ as implantation at this time does not improve prognosis. & III & A \\
\hline Recommendations for cardiac resynchronization therapy implantation in patients with heart failure & Class $^{\mathrm{a}}$ & Level $^{\mathrm{b}}$ \\
\hline $\begin{array}{l}\text { CRT is recommended for symptomatic patients with HF in sinus rhythm with a QRS duration } \geq 150 \text { msec and LBBB QRS morphology and } \\
\text { with LVEF } \leq 35 \% \text { despite OMT in order to improve symptoms and reduce morbidity and mortality. }\end{array}$ & I & $\mathbf{A}$ \\
\hline $\begin{array}{l}\text { CRT is recommended for symptomatic patients with } \mathrm{HF} \text { in sinus rhythm with a QRS duration of } 130-149 \text { msec and LBBB QRS } \\
\text { morphology and with LVEF } \leq 35 \% \text { despite OMT in order to improve symptoms and reduce morbidity and mortality. }\end{array}$ & $\mathbf{I}$ & B \\
\hline $\begin{array}{l}\text { CRT rather than RV pacing is recommended for patients with HFrEF regardless of NYHA Class who have an indication for ventricular } \\
\text { pacing and high degree } \mathrm{AV} \text { block in order to reduce morbidity. This includes patients with atrial fibrillation (see Section I0.I). }\end{array}$ & $\mathbf{I}$ & $\mathbf{A}$ \\
\hline CRT is contra-indicated in patients with a QRS duration $<130 \mathrm{msec}$ & III & A \\
\hline
\end{tabular}




\section{To do and not to do messages from the Guidelines (continued)}

\begin{tabular}{|c|c|c|}
\hline Not-recommended treatments of co-morbidities in patients with heart failure & Class $^{a}$ & Level $^{\mathrm{b}}$ \\
\hline $\begin{array}{l}\text { Adaptive servo-ventilation is not recommended in patients with } \mathrm{HFrEF} \text { and a predominant central sleep apnoea because of an } \\
\text { increased all-cause and cardiovascular mortality. }\end{array}$ & III & B \\
\hline Thiazolidinediones (glitazones) are not recommended in patients with HF, as they increase the risk of HF worsening and HF hospitalization. & III & A \\
\hline NSAIDs or COX-2 inhibitors are not recommended in patients with HF, as they increase the risk of HF worsening and HF hospitalization. & III & B \\
\hline Recommendations regarding diagnostic measurements in patients with suspected acute heart failure & Class $^{\mathrm{a}}$ & Level $^{\mathrm{b}}$ \\
\hline $\begin{array}{l}\text { Upon presentation a measurement of plasma natriuretic peptide level (BNP, NT-proBNP or MR-proANP) is recommended in all } \\
\text { patients with acute dyspnoea and suspected AHF to help in the differentiation of AHF from non-cardiac causes of acute dyspnoea. }\end{array}$ & I & $\mathbf{A}$ \\
\hline Recommendations for the management of patients with acute heart failure - pharmacotherapy & Class $^{a}$ & Level $^{\mathrm{b}}$ \\
\hline $\begin{array}{l}\text { Intravenous loop diuretics are recommended for all patients with AHF admitted with signs/symptoms of fluid overload to improve } \\
\text { symptoms. It is recommended to regularly monitor symptoms, urine output, renal function and electrolytes during use of i.v. diuretics. }\end{array}$ & I & C \\
\hline $\begin{array}{l}\text { In patients with new-onset } \mathrm{AHF} \text { or those with chronic, decompensated HF not receiving oral diuretics the initial recommended dose } \\
\text { should be } 20-40 \mathrm{mg} \text { i.v. furosemide (or equivalent); for those on chronic diuretic therapy, initial i.v. dose should be at least equivalent to } \\
\text { oral dose. }\end{array}$ & I & B \\
\hline $\begin{array}{l}\text { It is recommended to give diuretics either as intermittent boluses or a continuous infusion, and the dose and duration should be } \\
\text { adjusted according to the patients' symptoms and clinical status. }\end{array}$ & I & B \\
\hline Inotropic agents are not recommended unless the patient is symptomatically hypotensive or hypoperfused because of safety concern. & III & A \\
\hline Recommendations regarding management of patients with cardiogenic shock & Class $^{\mathrm{a}}$ & Level ${ }^{\mathrm{b}}$ \\
\hline In all patients with suspected cardiogenic shock, immediate ECG and echocardiography are recommended. & $\mathbf{I}$ & C \\
\hline $\begin{array}{l}\text { All patients with cardiogenic shock should be rapidly transferred to a tertiary care centre which has a } 24 / 7 \text { service of cardiac } \\
\text { catheterization, and a dedicated ICU/CCU with availability of short-term mechanical circulatory support. }\end{array}$ & I & c \\
\hline Recommendations regarding oral evidence-based disease-modifying therapies in patients with acute heart failure & Class $^{a}$ & Level ${ }^{\mathrm{b}}$ \\
\hline $\begin{array}{l}\text { In case of worsening of chronic HFrEF, every attempt should be made to continue evidence-based, disease-modifying therapies, in the } \\
\text { absence of haemodynamic instability or contra-indications. }\end{array}$ & I & C \\
\hline Recommendations for exercise, multidisciplinary management, and monitoring of patients with heart failure & Class $^{a}$ & Level $^{\mathrm{b}}$ \\
\hline It is recommended that regular aerobic exercise is encouraged in patients with $\mathrm{HF}$ to improve functional capacity and symptoms. & $\mathbf{I}$ & A \\
\hline It is recommended that regular aerobic exercise is encouraged in stable patients with HFrEF to reduce the risk of HF hospitalization. & $\mathbf{I}$ & A \\
\hline $\begin{array}{l}\text { It is recommended that patients with HF are enrolled in a multidisciplinary care management programme to reduce the risk of } \mathrm{HF} \\
\text { hospitalization and mortality. }\end{array}$ & I & A \\
\hline
\end{tabular}

ACE-I = angiotensin-converting enzyme inhibitor; $\mathrm{AHF}=$ acute heart failure; $\mathrm{ARB}=$ angiotensin receptor blocker; $\mathrm{AST}=$ aspartate aminotransferase; $\mathrm{AV}=$ atrio-ventricular; $\mathrm{BNP}=\mathrm{B}$-type natriuretic peptide; $\mathrm{CCU}=$ coronary care unit; $\mathrm{COX}-2=$ cyclooxygenase $2 ; \mathrm{CRT}=$ cardiac resynchronization therapy; $\mathrm{CT}=$ computed tomography; $\mathrm{DCM}=$ dilated cardiomyopathy; $\mathrm{ECG}=$ electrocardiogram; $\mathrm{HF}=$ heart failure; $\mathrm{HFmrEF}=$ heart failure with mid-range ejection fraction; $\mathrm{HFpEF}=$ heart failure with preserved ejection fraction; $\mathrm{HFrEF}=$ heart failure with reduced ejection fraction; ICD = implantable cardioverter-defibrillator; ICU = intensive care unit; i.v. = intravenous; $L B B B=$ left bundle branch block; $L V=$ left ventricular; $L V E F=$ left ventricular ejection fraction; $\mathrm{MI}=$ myocardial infarction; $M R A=$ mineralocorticoid receptor antagonist; $M R$-proANP = midregional pro A-type natriuretic peptide; NSAIDs = non-steroidal anti-inflammatory drugs; NT-proBNP = N-terminal pro-B type natriuretic peptide; NYHA = New York Heart Association; OMT = optimal medical therapy; $\mathrm{QRS}=\mathrm{Q}, \mathrm{R}$, and $\mathrm{S}$ waves (combination of three of the graphical deflections); $\mathrm{RV}=$ right ventricular; TTE $=$ transthoracic echocardiography.

${ }^{a}$ Class of recommendation.

'Level of evidence

'Or ARB if ACEl is not tolerated/contra-indicated.

\section{Web Addenda}

All Web figures and Web tables are available in the Web addenda, available at European Heart Journal online and also via the ESC website. 


\section{Appendix}

ESC Committee for Practice Guidelines (CPG): Jose Luis Zamorano (Chairperson) (Spain), Victor Aboyans (France), Stephan Achenbach (Germany), Stefan Agewall (Norway), Lina Badimon (Spain), Gonzalo Barón-Esquivias (Spain), Helmut Baumgartner (Germany), Jeroen J. Bax (The Netherlands), Héctor Bueno (Spain), Scipione Carerj (Italy), Veronica Dean (France), Çetin Erol (Turkey), Donna Fitzsimons (UK), Oliver Gaemperli (Switzerland), Paulus Kirchhof (UK/Germany), Philippe Kolh (Belgium), Patrizio Lancellotti (Belgium), Gregory Y. H. Lip (UK), Petros Nihoyannopoulos (UK), Massimo F. Piepoli (Italy), Piotr Ponikowski (Poland), Marco Roffi (Switzerland), Adam Torbicki (Poland), António Vaz Carneiro (Portugal), Stephan Windecker (Switzerland).

ESC National Cardiac Societies actively involved in the review process of the 2016 ESC Guidelines for the diagnosis and treatment of acute and chronic heart failure

Armenia: Armenian Cardiologists Association, Hamayak S. Sisakian; Azerbaijan: Azerbaijan Society of Cardiology, Elnur Isayev; Belarus: Belorussian Scientific Society of Cardiologists, Alena Kurlianskaya; Belgium: Belgian Society of Cardiology, Wilfried Mullens; Bulgaria: Bulgarian Society of Cardiology, Mariya Tokmakova; Cyprus: Cyprus Society of Cardiology, Petros Agathangelou; Czech Republic: Czech Society of Cardiology, Vojtech Melenovsky; Denmark: Danish Society of Cardiology, Henrik Wiggers; Egypt: Egyptian Society of Cardiology, Mahmoud Hassanein; Estonia: Estonian Society of Cardiology, Tiina Uuetoa; Finland: Finnish Cardiac Society, Jyri Lommi; Former Yugoslav Republic of Macedonia: Macedonian FYR Society of Cardiology, Elizabeta Srbinovska Kostovska; France: French Society of Cardiology, Yves Juillière; Georgia: Georgian Society of Cardiology, Alexander
Aladashvili; Germany: German Cardiac Society, Andreas Luchner; Greece: Hellenic Cardiological Society, Christina Chrysohoou; Hungary: Hungarian Society of Cardiology, Noémi Nyolczas; Iceland: Icelandic Society of Cardiology, Gestur Thorgeirsson; Israel: Israel Heart Society, Jean Marc Weinstein; Italy: Italian Federation of Cardiology, Andrea Di Lenarda; Kazakhstan: Association of Cardiologists of Kazakhstan, Nazipa Aidargaliyeva; Kosovo: Kosovo Society of Cardiology, Gani Bajraktari; Kyrgyzstan: Kyrgyz Society of Cardiology, Medet Beishenkulov; Latvia: Latvian Society of Cardiology, Ginta Kamzola; Lebanon: Lebanese Society of Cardiology, Tony Abdel-Massih; Lithuania: Lithuanian Society of Cardiology, Jelena Čelutkiene; Luxembourg: Luxembourg Society of Cardiology, Stéphanie Noppe; Malta: Maltese Cardiac Society, Andrew Cassar; Moldova: Moldavian Society of Cardiology, Eleonora Vataman; Morocco: Moroccan Society of Cardiology, Saadia AbirKhalil; The Netherlands: Netherlands Society of Cardiology, Petra van Pol; Norway: Norwegian Society of Cardiology, Rune Mo; Poland: Polish Cardiac Society, Ewa Straburzyńska-Migaj; Portugal: Portuguese Society of Cardiology, Cândida Fonseca; Romania: Romanian Society of Cardiology, Ovidiu Chioncel; Russian Federation: Russian Society of Cardiology, Evgeny Shlyakhto; San Marino: San Marino Society of Cardiology, Marco Zavatta; Serbia: Cardiology Society of Serbia, Petar Otasevic; Slovakia: Slovak Society of Cardiology, Eva Goncalvesová; Slovenia: Slovenian Society of Cardiology, Mitja Lainscak; Spain: Spanish Society of Cardiology, Beatriz Díaz Molina; Sweden: Swedish Society of Cardiology, Maria Schaufelberger; Switzerland: Swiss Society of Cardiology, Thomas Suter; Turkey: Turkish Society of Cardiology, Mehmet Birhan Yılmaz; Ukraine: Ukrainian Association of Cardiology, Leonid Voronkov; United Kingdom: British Cardiovascular Society, Ceri Davies.

The CME text '2016 ESC Guidelines for the diagnosis and treatment of acute and chronic heart failure' is accredited by the European Board for Accreditation in Cardiology (EBAC). EBAC works according to the quality standards of the European Accreditation Council for Continuing Medical Education (EACCME), which is an institution of the European Union of Medical Specialists (UEMS). In compliance with EBAC/EACCME Guidelines, all authors participating in this programme have disclosed any potential conflicts of interest that might cause a bias in the article. The Organizing Committee is responsible for ensuring that all potential conflicts of interest relevant to the programme are declared to the participants prior to the CME activities.

CME questions for this article are available at: European Heart Journal http://www.oxforde-learning.com/eurheartj and European Society of Cardiology http://www. escardio.org/guidelines

\section{References}

1. Filippatos G, Khan SS, Ambrosy AP, Cleland JGF, Collins SP, Lam CSP, Angermann CE, Ertl G, Dahlström U, Hu D, Dickstein K, Perrone S V, Ghadanfar M, Bermann G, Noe A, Schweizer A, Maier T, Gheorghiade M. International REgistry to assess medical Practice with IOngitudinal obseRvation for Treatment of Heart Failure (REPORT-HF): rationale for and design of a global registry. Eur J Heart Fail 2015;17:527-533.

2. The CONSENSUS Trial Study Group. Effects of enalapril on mortality in severe congestive heart failure. Results of the Cooperative North Scandinavian Enalapril Survival Study (CONSENSUS). N Engl J Med 1987;316:1429-1435.

3. McMurray JJ J. Improving outcomes in heart failure: a personal perspective. Eur Heart J 2015;36:3467-3470.

4. Wang TJ. Natural history of asymptomatic left ventricular systolic dysfunction in the community. Circulation 2003;108:977-982.

5. The SOLVD Investigators. Effect of enalapril on mortality and the development of heart failure in asymptomatic patients with reduced left ventricular ejection fractions. N Engl J Med 1992;327:685-691.

6. Butler J, Fonarow GC, Zile MR, Lam CS, Roessig L, Schelbert EB, Shah SJ, Ahmed A, Bonow RO, Cleland JGF, Cody RJ, Chioncel O, Collins SP, Dunnmon P, Filippatos G, Lefkowitz MP, Marti CN, McMurray J], Misselwitz F,
Nodari S, O'Connor C, Pfeffer MA, Pieske B, Pitt B, Rosano G, Sabbah HN, Senni M, Solomon SD, Stockbridge N, Teerlink JR, Georgiopoulou VV, Gheorghiade $M$. Developing therapies for heart failure with preserved ejection fraction: current state and future directions. JACC Heart Fail 2014;2:97-112.

7. McMurray JJ V, Adamopoulos S, Anker SD, Auricchio A, Böhm M, Dickstein K, Falk V, Filippatos G, Fonseca C, Gomez-Sanchez MA, Jaarsma T, Køber L, Lip GYH, Maggioni A Pietro, Parkhomenko A, Pieske BM, Popescu BA, Rønnevik PK, Rutten FH, Schwitter J, Seferovic P, Stepinska J, Trindade PT, Voors AA, Zannad F, Zeiher A, Bax JJ, Baumgartner H, Ceconi C, Dean V, Deaton C, Fagard R, Funck-Brentano C, Hasdai D, Hoes A, Kirchhof P, Knuuti J, Kolh P, McDonagh T, Moulin C, Reiner Z, Sechtem U, Sirnes PA, Tendera M, Torbicki A, Vahanian A, Windecker S, Bonet LA, Avraamides P, Ben Lamin HA, Brignole M, Coca A, Cowburn P, Dargie H, Elliott P, Flachskampf FA, Guida GF, Hardman S, lung B, Merkely B, Mueller C, Nanas JN, Nielsen OW, Orn S, Parissis JT, Ponikowski P, Members AF, McMurray JJ V, Adamopoulos S, Anker SD, Auricchio A, Böhm M, Dickstein K, Falk V, Filippatos G, Fonseca C, Gomez-Sanchez MA, Jaarsma T, Køber L, Lip GYH, Maggioni A Pietro, Parkhomenko A, Pieske BM, Popescu BA, Rønnevik PK, Rutten FH, Schwitter J, Seferovic P, Stepinska J, Trindade PT, Voors AA, Zannad F, Zeiher A, Guidelines ESCC for P, Reviewers D. ESC Guidelines for the diagnosis and treatment of acute and chronic heart failure 2012: The Task Force for the Diagnosis and Treatment of 
Acute and Chronic Heart Failure 2012 of the European Society of Cardiology. Developed in collaboration with the Heart. Eur J Heart Fail 2012;14:803-869.

8. McMurray JJ V. Clinical practice. Systolic heart failure. N Engl J Med 2010;3623: $228-238$.

9. Chen J, Normand S-LT, Wang Y, Krumholz HM. National and regional trends in heart failure hospitalization and mortality rates for Medicare beneficiaries, 1998 2008. JAMA 2011;306:1669-1678.

10. Dunlay SM, Redfield MM, Weston SA, Therneau TM, Hall Long K, Shah ND, Roger VL. Hospitalizations after heart failure diagnosis a community perspective. J Am Coll Cardiol 2009;54:1695-1702.

11. Metra M, Ponikowski P, Dickstein K, McMurray JJ V, Gavazzi A, Bergh C-H, Fraser AG, Jaarsma T, Pitsis A, Mohacsi P, Böhm M, Anker S, Dargie H, Brutsaert D, Komajda M. Advanced chronic heart failure: a position statement from the Study Group on Advanced Heart Failure of the Heart Failure Association of the European Society of Cardiology. Eur J Heart Fail 9:684-694.

12. Yancy CW, Jessup M, Bozkurt B, Butler J, Casey DE, Drazner MH, Fonarow GC, Geraci SA, Horwich T, Januzzi JL, Johnson MR, Kasper EK, Levy WC, Masoudi FA, McBride PE, McMurray JJ V, Mitchell JE, Peterson PN, Riegel B, Sam F, Stevenson LW, Tang WHW, Tsai EJ, Wilkoff BL. 2013 ACCF/AHA Guideline for the Management of Heart Failure: executive summary: a report of the American College of Cardiology Foundation/American Heart Association Task Force on Practice Guidelines. Circulation 2013;128:1810-1852.

13. Killip T 3rd, Kimball JT. Treatment of myocardial infarction in a coronary care unit. A two year experience with 250 patients. Am J Cardiol 1967;20:457-464.

14. Mosterd A, Hoes AW. Clinical epidemiology of heart failure. Heart 2007;93: 1137-1146.

15. Redfield MM, Jacobsen SJ, Burnett JC, Mahoney DW, Bailey KR, Rodeheffer RJ. Burden of systolic and diastolic ventricular dysfunction in the community: appreciating the scope of the heart failure epidemic. JAMA 2003;289:194-202.

16. Bleumink GS, Knetsch AM, Sturkenboom MCJM, Straus SMJM, Hofman A, Deckers JW, Witteman JCM, Stricker BHC. Quantifying the heart failure epidemic: prevalence, incidence rate, lifetime risk and prognosis of heart failure The Rotterdam Study. Eur Heart J England; 2004;25:1614-1619.

17. Ceia F, Fonseca C, Mota T, Morais H, Matias F, De Sousa A, Oliveira AG. Prevalence of chronic heart failure in Southwestern Europe: the EPICA study. Eur J Heart Fail 2002;4:531-539.

18. van Riet EES, Hoes AW, Limburg A, Landman MAJ, van der Hoeven H, Rutten FH. Prevalence of unrecognized heart failure in older persons with shortness of breath on exertion. Eur J Heart Fail 2014;16:772-777.

19. Filippatos G, Parissis JT. Heart failure diagnosis and prognosis in the elderly: the proof of the pudding is in the eating. Eur J Heart Fail 2011;13:467-471.

20. Rutten FH, Cramer M-JM, Grobbee DE, Sachs APE, Kirkels JH, Lammers J-WJ, Hoes AW. Unrecognized heart failure in elderly patients with stable chronic obstructive pulmonary disease. Eur Heart J 2005;26:1887-1894.

21. Boonman-de Winter LJM, Rutten FH, Cramer MJM, Landman MJ, Liem AH, Rutten GEHM, Hoes AW. High prevalence of previously unknown heart failure and left ventricular dysfunction in patients with type 2 diabetes. Diabetologia 2012;55:2154-2162.

22. van Riet EES, Hoes AW, Wagenaar KP, Limburg A, Landman MAJ, Rutten FH. Epidemiology of heart failure: the prevalence of heart failure and ventricular dysfunction in older adults over time. A systematic review. Eur J Heart Fail 2016;

23. Abhayaratna WP, Smith WT, Becker NG, Marwick TH, Jeffery IM, McGill DA. Prevalence of heart failure and systolic ventricular dysfunction in older Australians: the Canberra Heart Study. Med J Aust 2006;184:151-154.

24. Tiller D, Russ M, Greiser KH, Nuding S, Ebelt H, Kluttig A, Kors JA, Thiery J, Bruegel M, Haerting J, Werdan K. Prevalence of symptomatic heart failure with reduced and with normal ejection fraction in an elderly general populationthe CARLA study. PLoS One 2013;8:e59225.

25. Mureddu GF, Agabiti N, Rizzello V, Forastiere F, Latini R, Cesaroni G, Masson S, Cacciatore G, Colivicchi F, Uguccioni M, Perucci CA, Boccanelli A. Prevalence of preclinical and clinical heart failure in the elderly. A population-based study in Central Italy. Eur J Heart Fail 2012;14:718-729.

26. Badano LP, Albanese MC, De Biaggio P, Rozbowsky P, Miani D, Fresco C, Fioretti PM. Prevalence, clinical characteristics, quality of life, and prognosis of patients with congestive heart failure and isolated left ventricular diastolic dysfunction. J Am Soc Echocardiogr 2004;17:253-261.

27. Philbin EF, Rocco TA, Lindenmuth NW, Ulrich K, Jenkins PL. Systolic versus diastolic heart failure in community practice: clinical features, outcomes, and the use of angiotensin-converting enzyme inhibitors. Am J Med 2000;109:605-613.

28. Magaña-Serrano JA, Almahmeed W, Gomez E, Al-Shamiri M, Adgar D, Sosner P, Herpin D. Prevalence of heart failure with preserved ejection fraction in Latin American, Middle Eastern, and North African Regions in the I PREFER study (Identification of Patients With Heart Failure and PREserved Systolic Function: an epidemiological regional study. Am J Cardiol 2011;108:1289-1296.
29. Peyster E, Norman J, Domanski M. Prevalence and predictors of heart failure with preserved systolic function: community hospital admissions of a racially and gender diverse elderly population. J Card Fail 2004;10:49-54.

30. Hellermann JP, Jacobsen SJ, Reeder GS, Lopez-Jimenez F, Weston SA, Roger VL. Heart failure after myocardial infarction: prevalence of preserved left ventricular systolic function in the community. Am Heart / 2003;145:742-748.

31. Gerber Y, Weston SA, Redfield MM, Chamberlain AM, Manemann SM, Jiang R, Killian JM, Roger VL. A contemporary appraisal of the heart failure epidemic in Olmsted County, Minnesota, 2000 to 2010. JAMA Intern Med 2015;175: 996-1004.

32. Owan TE, Hodge DO, Herges RM, Jacobsen SJ, Roger VL, Redfield MM. Trends in prevalence and outcome of heart failure with preserved ejection fraction. N EnglJ Med 2006;355:251-259.

33. Meta-analysis Global Group in Chronic Heart Failure (MAGGIC). The survival of patients with heart failure with preserved or reduced left ventricular ejection fraction: an individual patient data meta-analysis. Eur Heart J 2012;33:1750-1757.

34. Lam CSP, Solomon SD. The middle child in heart failure: heart failure with midrange ejection fraction (40-50\%). Eur J Heart Fail 2014;16:1049-1055.

35. Maggioni AP, Dahlström U, Filippatos G, Chioncel O, Leiro MC, Drozdz J, Fruhwald F, Gullestad L, Logeart D, Fabbri G, Urso R, Metra M, Parissis ], Persson H, Ponikowski P, Rauchhaus M, Voors AA, Nielsen OW, Zannad F, Tavazzi L, Crespo Leiro M, Drozdz J, Fruhwald F, Gullestad L, Logeart D, Fabbri G, Urso R, Metra M, Parissis J, Persson H, Ponikowski P, Rauchhaus M, Voors AA, Nielsen OW, Zannad F, Tavazzi L. EURObservational Research Programme: regional differences and 1-year follow-up results of the Heart Failure Pilot Survey (ESC-HF Pilot). Eur J Heart Fail 2013;15:808-817.

36. Pocock SJ, Ariti CA, McMurray JJV, Maggioni A, Køber L, Squire IB, Swedberg K, Dobson J, Poppe KK, Whalley GA, Doughty RN. Predicting survival in heart failure: a risk score based on 39372 patients from 30 studies. Eur Heart J 2013;34: 1404-1413.

37. Rahimi K, Bennett D, Conrad N, Williams TM, Basu J, Dwight J, Woodward M, Patel A, McMurray J, MacMahon S. Risk prediction in patients with heart failure. JACC Heart Fail 2014;2:440-446.

38. Ouwerkerk W, Voors AA, Zwinderman AH. Factors influencing the predictive power of models for predicting mortality and/or heart-failure hospitalization in patients with heart failure. JACC Heart Fail 2014;2:429-436.

39. Lupón J, de Antonio M, Vila J, Peñafiel J, Galán A, Zamora E, Urrutia A, Bayes-Genis A. Development of a novel heart failure risk tool: the Barcelona bioheart failure risk calculator (BCN bio-HF calculator). PLoS One 2014;9:e85466.

40. Levy WC. The Seattle Heart Failure Model: prediction of survival in heart failure. Circulation 2006;113:1424-1433.

41. Mozaffarian D, Anker SD, Anand I, Linker DT, Sullivan MD, Cleland JGF, Carson PE, Maggioni AP, Mann DL, Pitt B, Poole-Wilson PA, Levy WC. Prediction of mode of death in heart failure: the Seattle Heart Failure Model. Circulation 2007; 116:392-398.

42. Davie P, Francis CM, Caruana L, Sutherland GR, McMurray JJ. Assessing diagnosis in heart failure: which features are any use? QJM 1997;90:335-339.

43. Mant J, Doust J, Roalfe A, Barton P, Cowie MR, Glasziou P, Mant D, McManus RJ, Holder R, Deeks J, Fletcher K, Qume M, Sohanpal S, Sanders S, Hobbs FDR. Systematic review and individual patient data meta-analysis of diagnosis of heart failure, with modelling of implications of different diagnostic strategies in primary care. Health Technol Assess 2009;13:1-207, iii.

44. Oudejans I, Mosterd A, Bloemen JA, Valk MJ, Van Velzen E, Wielders JP, Zuithoff NP, Rutten FH, Hoes AW. Clinical evaluation of geriatric outpatients with suspected heart failure: value of symptoms, signs, and additional tests. Eur J Heart Fail 2011;13:518-527.

45. Fonseca C. Diagnosis of heart failure in primary care. Heart Fail Rev 2006;11 95-107.

46. Kelder JC, Cramer MJ, van Wijngaarden J, van Tooren R, Mosterd A, Moons KGM, Lammers JW, Cowie MR, Grobbee DE, Hoes AW. The diagnostic value of physical examination and additional testing in primary care patients with suspected heart failure. Circulation 2011;124:2865-2873.

47. Boonman-de Winter LJM, Rutten FH, Cramer MJ, Landman MJ, Zuithoff NPA Liem AH, Hoes AW. Efficiently screening heart failure in patients with type 2 diabetes. Eur J Heart Fail 2015;17:187-195.

48. Rutten FH, Moons KGM, Cramer M-JM, Grobbee DE, Zuithoff NPA, Lammers J-WJ, Hoes AW. Recognising heart failure in elderly patients with stable chronic obstructive pulmonary disease in primary care: cross sectional diagnostic study. BM/ 2005;331:1379.

49. Hawkins NM, Petrie MC, Jhund PS, Chalmers GW, Dunn FG, McMurray JJV. Heart failure and chronic obstructive pulmonary disease: diagnostic pitfalls and epidemiology. Eur J Heart Fail 2009;11:130-139.

50. Daniels LB, Clopton P, Bhalla V, Krishnaswamy P, Nowak RM, McCord J, Hollander JE, Duc P, Omland T, Storrow AB, Abraham WT, Wu AHB, Steg PG, Westheim A, Knudsen CW, Perez A, Kazanegra R, Herrmann HC, 
McCullough PA, Maisel AS. How obesity affects the cut-points for B-type natriuretic peptide in the diagnosis of acute heart failure. Results from the Breathing Not Properly Multinational Study. Am Heart J 2006;151:999-1005.

51. Wong CM, Hawkins NM, Jhund PS, MacDonald MR, Solomon SD, Granger CB, Yusuf S, Pfeffer MA, Swedberg K, Petrie MC, McMurray JJ V. Clinical characteristics and outcomes of young and very young adults with heart failure: the CHARM programme (Candesartan in Heart Failure Assessment of Reduction in Mortality and Morbidity). J Am Coll Cardiol 2013;62:1845-1854.

52. Wong CM, Hawkins NM, Petrie MC, Jhund PS, Gardner RS, Ariti CA, Poppe KK, Earle N, Whalley GA, Squire IB, Doughty RN, McMurray JJV. Heart failure in younger patients: the Meta-analysis Global Group in Chronic Heart Failure (MAGGIC). Eur Heart J 2014;35:2714-2721.

53. Thibodeau JT, Turer AT, Gualano SK, Ayers CR, Velez-Martinez M, Mishkin JD, Patel PC, Mammen PPA, Markham DW, Levine BD, Drazner MH. Characterization of a novel symptom of advanced heart failure: bendopnea. JACC Heart Fail 2014;2:24-31.

54. Roberts E, Ludman AJ, Dworzynski K, Al-Mohammad A, Cowie MR, McMurray JJV, Mant J. The diagnostic accuracy of the natriuretic peptides in heart failure: systematic review and diagnostic meta-analysis in the acute care setting. BMJ 2015;350:h910.

55. Maisel A, Mueller C, Adams K, Anker SD, Aspromonte N, Cleland JGF, Cohen-Solal A, Dahlstrom U, DeMaria A, DiSomma S, Filippatos GS, Fonarow GC, Jourdain P, Komajda M, Liu PP, McDonagh T, McDonald K, Mebazaa A, Nieminen MS, Peacock WF, Tubaro M, Valle R, Vanderhyden M, Yancy CW, Zannad F, Braunwald E. State of the art: using natriuretic peptide levels in clinical practice. Eur J Heart Fail 2008;10:824-839.

56. Zaphiriou A, Robb S, Murray-Thomas T, Mendez G, Fox K, McDonagh T, Hardman SMC, Dargie HJ, Cowie MR. The diagnostic accuracy of plasma BNP and NTproBNP in patients referred from primary care with suspected heart failure: results of the UK natriuretic peptide study. Eur J Heart Fail 2005;7:537-541.

57. Fuat A, Murphy JJ, Hungin APS, Curry J, Mehrzad AA, Hetherington A, Johnston Jl, Smellie WSA, Duffy V, Cawley P. The diagnostic accuracy and utility of a B-type natriuretic peptide test in a community population of patients with suspected heart failure. Br J Gen Pract 2006;56:327-333.

58. Yamamoto K, Burnett JC, Bermudez EA, Jougasaki M, Bailey KR, Redfield MM. Clinical criteria and biochemical markers for the detection of systolic dysfunction. J Card Fail 2000;6:194-200.

59. Cowie MR, Struthers AD, Wood DA, Coats AJ, Thompson SG, Poole-Wilson PA, Sutton GC. Value of natriuretic peptides in assessment of patients with possible new heart failure in primary care. Lancet 1997;350:1349-1353.

60. Krishnaswamy P, Lubien E, Clopton P, Koon J, Kazanegra R, Wanner E, Gardetto N, Garcia A, DeMaria A, Maisel AS. Utility of B-natriuretic peptide levels in identifying patients with left ventricular systolic or diastolic dysfunction. Am J Med 2001;111:274-279.

61. Kelder JC, Cramer MJ, Verweij WM, Grobbee DE, Hoes AW. Clinical utility of three B-type natriuretic peptide assays for the initial diagnostic assessment of new slow-onset heart failure. J Card Fail 2011;17:729-734.

62. Madamanchi C, Alhosaini H, Sumida A, Runge MS. Obesity and natriuretic peptides, BNP and NT-proBNP: Mechanisms and diagnostic implications for heart failure. Int J Cardiol 2014;176:611-617.

63. Davie AP, Francis CM, Love MP, Caruana L, Starkey IR, Shaw TR, Sutherland GR, McMurray JJ. Value of the electrocardiogram in identifying heart failure due to left ventricular systolic dysfunction. BMJ 1996;312:222.

64. Thomas JT, Kelly RF, Thomas SJ, Stamos TD, Albasha K, Parrillo JE, Calvin JE. Utility of history, physical examination, electrocardiogram, and chest radiograph for differentiating normal from decreased systolic function in patients with heart failure. Am J Med 2002;112:437-445.

65. Paulus WJ, Tschope C, Sanderson JE, Rusconi C, Flachskampf FA, Rademakers FE, Marino P, Smiseth OA, De Keulenaer G, Leite-Moreira AF, Borbely A, Edes I, Handoko ML, Heymans S, Pezzali N, Pieske B, Dickstein K, Fraser AG, Brutsaert DL. How to diagnose diastolic heart failure: a consensus statement on the diagnosis of heart failure with normal left ventricular ejection fraction by the Heart Failure and Echocardiography Associations of the European Society of Cardiology. Eur Heart J 2007;28:2539-2550.

66. Marwick TH, Raman SV, Carrió I, Bax JJ. Recent developments in heart failure imaging. JACC Cardiovasc Imaging 2010;3:429-439.

67. Dokainish H, Nguyen JS, Bobek J, Goswami R, Lakkis NM. Assessment of the American Society of Echocardiography-European Association of Echocardiography guidelines for diastolic function in patients with depressed ejection fraction: an echocardiographic and invasive haemodynamic study. Eur J Echocardiogr 2011; 12:857-864.

68. Kirkpatrick JN, Vannan MA, Narula J, Lang RM. Echocardiography in heart failure: applications, utility, and new horizons. J Am Coll Cardiol 2007;50:381-396.

69. Nagueh SF, Bhatt R, Vivo RP, Krim SR, Sarvari SI, Russell K, Edvardsen T, Smiseth OA, Estep JD. Echocardiographic evaluation of hemodynamics in patients with decompensated systolic heart failure. Circ Cardiovasc Imaging 2011;4: 220-227.

70. Caballero L, Kou S, Dulgheru R, Gonjilashvili N, Athanassopoulos GD, Barone D, Baroni M, Cardim N, Gomez de Diego JJ, Oliva MJ, Hagendorff A, Hristova K, Lopez T, Magne J, Martinez C, de la Morena G, Popescu BA, Penicka M, Ozyigit T, Rodrigo Carbonero JD, Salustri A, Van De Veire N, Von Bardeleben RS, Vinereanu D, Voigt J-U, Zamorano JL, Bernard A, Donal E, Lang RM, Badano LP, Lancellotti P. Echocardiographic reference ranges for normal cardiac Doppler data: results from the NORRE Study. Eur Heart J Cardiovasc Imaging 2015;16:1031-1041.

71. Garbi M, McDonagh T, Cosyns B, Bucciarelli-Ducci C, Edvardsen T, Kitsiou A, Nieman K, Lancellotti P. Appropriateness criteria for cardiovascular imaging use in heart failure: report of literature review. Eur Heart J Cardiovasc Imaging 2015;16: 147-153.

72. Lang RM, Badano LP, Mor-Avi V, Afilalo J, Armstrong A, Ernande L, Flachskampf FA, Foster E, Goldstein SA, Kuznetsova T, Lancellotti P, Muraru D, Picard MH, Rietzschel ER, Rudski L, Spencer KT, Tsang W, Voigt J-U. Recommendations for cardiac chamber quantification by echocardiography in adults: an update from the American Society of Echocardiography and the European Association of Cardiovascular Imaging. Eur Heart J Cardiovasc Imaging 2015;16: 233-270.

73. Gimelli A, Lancellotti P, Badano LP, Lombardi M, Gerber B, Plein S, Neglia D, Edvardsen T, Kitsiou A, Scholte AJHA, Schroder S, Cosyns B, Gargiulo P, Zamorano JL, Perrone-Filardi P. Non-invasive cardiac imaging evaluation of patients with chronic systolic heart failure: a report from the European Association of Cardiovascular Imaging (EACVI). Eur Heart J 2014;35:3417-3425.

74. Voigt J-U, Pedrizzetti G, Lysyansky P, Marwick TH, Houle H, Baumann R, Pedri S, Ito Y, Abe Y, Metz S, Song JH, Hamilton J, Sengupta PP, Kolias TJ, d'Hooge J, Aurigemma GP, Thomas JD, Badano LP. Definitions for a common standard for 2D speckle tracking echocardiography: consensus document of the EACVI/ ASE/Industry Task Force to standardize deformation imaging. Eur Heart J Cardiovasc Imaging 2015;16:1-11.

75. Ewald B, Ewald D, Thakkinstian A, Attia J. Meta-analysis of B type natriuretic peptide and $\mathrm{N}$-terminal pro $\mathrm{B}$ natriuretic peptide in the diagnosis of clinical heart failure and population screening for left ventricular systolic dysfunction. Intern Med J 2008;38:101-113.

76. Doust JA, Glasziou PP, Pietrzak E, Dobson AJ. A systematic review of the diagnostic accuracy of natriuretic peptides for heart failure. Arch Intern Med 2004;164: 1978-1984.

77. Gustafsson F, Steensgaard-Hansen F, Badskjaer J, Poulsen AH, Corell P, Hildebrandt P. Diagnostic and prognostic performance of $\mathrm{N}$-terminal ProBNP in primary care patients with suspected heart failure. J Card Fail 2005;11:S15-S20.

78. Nielsen OW, Rasmussen V, Christensen NJ, Hansen JF. Neuroendocrine testing in community patients with heart disease: plasma $\mathrm{N}$-terminal proatrial natriuretic peptide predicts morbidity and mortality stronger than catecholamines and heart rate variability. Scand J Clin Lab Invest 2004;64:619-628.

79. Kelly JP, Mentz RJ, Mebazaa A, Voors AA, Butler J, Roessig L, Fiuzat M, Zannad F, Pitt B, O'Connor CM, Lam CSP. Patient selection in heart failure with preserved ejection fraction clinical trials. J Am Coll Cardiol 2015;65:1668-1682.

80. Cohen GI, Pietrolungo JF, Thomas JD, Klein AL. A practical guide to assessment of ventricular diastolic function using Doppler echocardiography. J Am Coll Cardiol 1996;27:1753-1760.

81. Gilman G, Nelson TA, Hansen WH, Khandheria BK, Ommen SR. Diastolic function: a sonographer's approach to the essential echocardiographic measurements of left ventricular diastolic function. J Am Soc Echocardiogr 2007;20:199-209.

82. Nagueh SF, Appleton CP, Gillebert TC, Marino PN, Oh JK, Smiseth OA, Waggoner AD, Flachskampf FA, Pellikka PA, Evangelisa A. Recommendations for the evaluation of left ventricular diastolic function by echocardiography. Eur J Echocardiogr 2009;10:165-193.

83. Nishimura RA, Appleton CP, Redfield MM, Ilstrup DM, Holmes DR, Tajik AJ. Noninvasive doppler echocardiographic evaluation of left ventricular filling pressures in patients with cardiomyopathies: a simultaneous Doppler echocardiographic and cardiac catheterization study. J Am Coll Cardiol 1996;28:1226-1233.

84. Ommen SR, Nishimura RA, Appleton CP, Miller FA, Oh JK, Redfield MM, Tajik AJ. Clinical utility of Doppler echocardiography and tissue doppler imaging in the estimation of left ventricular filling pressures: a comparative simultaneous Dopplercatheterization study. Circulation 2000;102:1788-1794.

85. Erdei T, Smiseth OA, Marino P, Fraser AG. A systematic review of diastolic stress tests in heart failure with preserved ejection fraction, with proposals from the EU-FP7 MEDIA study group. Eur J Heart Fail 2014;16:1345-1361.

86. Donal E, Lund LH, Oger E, Reynaud A, Schnell F, Persson H, Drouet E, Linde C, Daubert C, KaRen investigators. Value of exercise echocardiography in heart failure with preserved ejection fraction: a substudy from the KaRen study. Eur Heart J Cardiovasc Imaging 2016;17:106-113. 
87. Borlaug BA, Nishimura RA, Sorajja P, Lam CSP, Redfield MM. Exercise hemodynamics enhance diagnosis of early heart failure with preserved ejection fraction. Circ Heart Fail 2010;3:588-595

88. Elliott PM, Anastasakis A, Borger MA, Borggrefe M, Cecchi F, Charron P, Hagege AA, Lafont A, Limongelli G, Mahrholdt H, McKenna WJ, Mogensen J, Nihoyannopoulos P, Nistri S, Pieper PG, Pieske B, Rapezzi C, Rutten FH, Tillmanns C, Watkins H. 2014 ESC Guidelines on diagnosis and management of hypertrophic cardiomyopathy: the Task Force for the Diagnosis and Management of Hypertrophic Cardiomyopathy of the European Society of Cardiology (ESC). Eur Heart J 2014;35:2733-2779.

89. Cosyns B, Plein S, Nihoyanopoulos P, Smiseth O, Achenbach S, Andrade MJ, Pepi M, Ristic A, Imazio M, Paelinck B, Lancellotti P. European Association of Cardiovascular Imaging (EACVI) position paper: multimodality imaging in pericardial disease. Eur Heart J Cardiovasc Imaging 2015;16:12-31.

90. Caforio ALP, Pankuweit S, Arbustini E, Basso C, Gimeno-Blanes J, Felix SB, Fu M, Helio T, Heymans S, Jahns R, Klingel K, Linhart A, Maisch B, McKenna W, Mogensen J, Pinto YM, Ristic A, Schultheiss H-P, Seggewiss H, Tavazzi L, Thiene G, Yilmaz A, Charron P, Elliott PM. Current state of knowledge on aetiology, diagnosis, management, and therapy of myocarditis: a position statement of the European Society of Cardiology Working Group on Myocardial and Pericardial Diseases. Eur Heart J 2013;34:2636-2648.

91. Gonzalez JA, Kramer CM. Role of imaging techniques for diagnosis, prognosis and management of heart failure patients: cardiac magnetic resonance. Curr Heart Fail Rep 2015;12:276-283.

92. Peix A, Mesquita CT, Paez D, Pereira CC, Felix R, Gutierrez C, Jaimovich R, lanni BM, Soares J, Olaya P, Rodriguez MV, Flotats A, Giubbini R, Travin M, Garcia $E$ V. Nuclear medicine in the management of patients with heart failure: guidance from an expert panel of the International Atomic Energy Agency (IAEA). Nucl Med Commun 2014;35:818-823.

93. Cooper LT, Baughman KL, Feldman AM, Frustaci A, Jessup M, Kuhl U, Levine GN, Narula J, Starling RC, Towbin J, Virmani R. The role of endomyocardial biopsy in the management of cardiovascular disease: a scientific statement from the American Heart Association, the American College of Cardiology, and the European Society of Cardiology Endorsed by the Heart Failure Society of America and the Heart Failure Association of the European Society of Cardiology. Eur Heart J 2007;28:3076-3093.

94. Charron P, Arad M, Arbustini E, Basso C, Bilinska Z, Elliott P, Helio T, Keren A, McKenna WJ, Monserrat L, Pankuweit S, Perrot A, Rapezzi C, Ristic A, Seggewiss H, Van Langen I, Tavazzi L. Genetic counselling and testing in cardiomyopathies: a position statement of the European Society of Cardiology Working Group on Myocardial and Pericardial Diseases. Eur Heart J 2010;31:2715-2728.

95. Lang RM, Badano LP, Tsang W, Adams DH, Agricola E, Buck T, Faletra FF, Franke A, Hung J, Pérez de Isla L, Kamp O, Kasprzak JD, Lancellotti P, Marwick TH, McCulloch ML, Monaghan MJ, Nihoyannopoulos P, Pandian NG, Pellikka PA, Pepi M, Roberson DA, Shernan SK, Shirali GS, Sugeng L, Ten Cate FJ, Vannan MA, Zamorano JL, Zoghbi WA. EAE/ASE recommendations for image acquisition and display using three-dimensional echocardiography. J Am Soc Echocardiogr 2012;25:3-46.

96. Rudski LG, Lai WW, Afilalo J, Hua L, Handschumacher MD, Chandrasekaran K, Solomon SD, Louie EK, Schiller NB. Guidelines for the echocardiographic assessment of the right heart in adults: a report from the American Society of Echocardiography. Endorsed by the European Association of Echocardiography, a registered branch of the European Society of Cardiology, and the Canadian Society of Echocardiography. J Am Soc Echocardiogr 2010;23:685-713.

97. Galiè N, Humbert M, Vachiery J-L, Gibbs S, Lang I, Torbicki A, Simonneau G, Peacock A, Vonk Noordegraaf A, Beghetti M, Ghofrani A, Gomez Sanchez MA, Hansmann G, Klepetko W, Lancellotti P, Matucci M, McDonagh T, Pierard LA, Trindade PT, Zompatori M, Hoeper M. 2015 ESC/ERS Guidelines for the diagnosis and treatment of pulmonary hypertension. Eur Heart J 2015;37:ehv317.

98. Smith BCF, Dobson G, Dawson D, Charalampopoulos A, Grapsa J, Nihoyannopoulos P. Three-dimensional speckle tracking of the right ventricle: toward optimal quantification of right ventricular dysfunction in pulmonary hypertension. J Am Coll Cardiol 2014;64:41-51.

99. Sicari R, Nihoyannopoulos P, Evangelista A, Kasprzak J, Lancellotti P, Poldermans D, Voigt JU, Zamorano JL. Stress Echocardiography Expert Consensus Statement-Executive Summary: European Association of Echocardiography (EAE) (a registered branch of the ESC). Eur Heart J 2009;30:278-289.

100. Garnier F, Eicher J-C, Jazayeri S, Bertaux G, Bouchot O, Aho L-S, Wolf J-E, Laurent $G$. Usefulness and limitations of contractile reserve evaluation in patients with low-flow, low-gradient aortic stenosis eligible for cardiac resynchronization therapy. Eur J Heart Fail 2014;16:648-654.

101. Hundley WG, Bluemke DA, Finn JP, Flamm SD, Fogel MA, Friedrich MG, Ho VB, Jerosch-Herold M, Kramer CM, Manning WJ, Patel M, Pohost GM, Stillman AE, White RD, Woodard PK, Harrington RA, Anderson JL, Bates ER, Bridges CR, Eisenberg MJ, Ferrari VA, Grines CL, Hlatky MA, Jacobs AK, Kaul S,
Lichtenberg RC, Lindner JR, Moliterno DJ, Mukherjee D, Rosenson RS, Schofield RS, Shubrooks SJ, Stein JH, Tracy CM, Weitz HH, Wesley DJ. ACCF/ ACR/AHA/NASCI/SCMR 2010 expert consensus document on cardiovascular magnetic resonance: a report of the american college of cardiology foundation task force on expert consensus documents. Circulation 2010;121:2462-2508.

102. Kilner PJ, Geva T, Kaemmerer H, Trindade PT, Schwitter J, Webb GD. Recommendations for cardiovascular magnetic resonance in adults with congenital heart disease from the respective working groups of the European Society of Cardiology. Eur Heart J 2010;31:794-805.

103. Moon JC, Messroghli DR, Kellman P, Piechnik SK, Robson MD, Ugander M, Gatehouse PD, Arai AE, Friedrich MG, Neubauer S, Schulz-Menger J, Schelbert EB. Myocardial T1 mapping and extracellular volume quantification: a Society for Cardiovascular Magnetic Resonance (SCMR) and CMR Working Group of the European Society of Cardiology consensus statement. J Cardiovasc Magn Reson 2013;15:92.

104. Yoshida A, Ishibashi-Ueda H, Yamada N, Kanzaki H, Hasegawa T, Takahama H, Amaki M, Asakura M, Kitakaze M. Direct comparison of the diagnostic capability of cardiac magnetic resonance and endomyocardial biopsy in patients with heart failure. Eur J Heart Fail 2013;15:166-175.

105. Bonow RO, Castelvecchio S, Panza JA, Berman DS, Velazquez EJ, Michler RE, She L, Holly TA, Desvigne-Nickens P, Kosevic D, Rajda M, Chrzanowski L, Deja M, Lee KL, White H, Oh JK, Doenst T, Hill JA, Rouleau JL, Menicanti L. Severity of remodeling, myocardial viability, and survival in ischemic LV dysfunction after surgical revascularization. JACC Cardiovasc Imaging 2015;8:1121-1129.

106. McDiarmid AK, Loh H, Nikitin N, Cleland JG, Ball SG, Greenwood JP, Plein S, Sparrow P. Predictive power of late gadolinium enhancement for myocardial recovery in chronic ischaemic heart failure: a HEART sub-study. ESC Heart Fail 2014; 1:146-153.

107. Cleland JGF, Calvert M, Freemantle N, Arrow Y, Ball SG, Bonser RS, Chattopadhyay S, Norell MS, Pennell DJ, Senior R. The heart failure revascularisation trial (HEART). Eur J Heart Fail 2011;13:227-233.

108. Haneder S, Kucharczyk W, Schoenberg SO, Michaely HJ. Safety of magnetic resonance contrast media: a review with special focus on nephrogenic systemic fibrosis. Top Magn Reson Imaging 2015;24:57-65.

109. Beller GA, Heede RC. SPECT imaging for detecting coronary artery disease and determining prognosis by noninvasive assessment of myocardial perfusion and myocardial viability. J Cardiovasc Transl Res 2011;4:416-424.

110. González-López E, Gallego-Delgado M, Guzzo-Merello G, de Haro-Del Moral FJ, Cobo-Marcos M, Robles C, Bornstein B, Salas C, Lara-Pezzi E, Alonso-Pulpon L, Garcia-Pavia P. Wild-type transthyretin amyloidosis as a cause of heart failure with preserved ejection fraction. Eur Heart J 2015;36:2585-2594.

111. Beanlands RSB, Nichol G, Huszti E, Humen D, Racine N, Freeman $M$, Gulenchyn KY, Garrard L, deKemp R, Guo A, Ruddy TD, Benard F, Lamy A, Iwanochko RM. F-18-fluorodeoxyglucose positron emission tomography imaging-assisted management of patients with severe left ventricular dysfunction and suspected coronary disease: a randomized, controlled trial (PARR-2). J Am Coll Cardiol 2007; 50:2002-2012.

112. Windecker S, Kolh P, Alfonso F, Collet J-P, Cremer J, Falk V, Filippatos G, Hamm C, Head SJ, Juni P, Kappetein AP, Kastrati A, Knuuti J, Landmesser U, Laufer G, Neumann F-J, Richter DJ, Schauerte P, Sousa Uva M, Stefanini GG, Taggart DP, Torracca L, Valgimigli M, Wijns W. 2014 ESC/EACTS Guidelines on myocardial revascularization: the Task Force on Myocardial Revascularization of the European Society of Cardiology (ESC) and the European Association for Cardio-Thoracic Surgery (EACTS). Developed with the special contribution of the European Association of Percutaneous Cardiovascular Interventions (EAPCl). Eur Heart J 2014;35:2541-2619.

113. Montalescot G, Sechtem U, Achenbach S, Andreotti F, Arden C, Budaj A, Bugiardini R, Crea F, Cuisset T, Di Mario C, Ferreira JR, Gersh BJ, Gitt AK, Hulot J-S, Marx N, Opie LH, Pfisterer M, Prescott E, Ruschitzka F, Sabaté M, Senior R, Taggart DP, van der Wall EE, Vrints CJM, Zamorano JL, Baumgartner $H$, Bax J], Bueno $H$, Dean V, Deaton C, Erol C, Fagard R, Ferrari R, Hasdai D, Hoes AW, Kirchhof P, Knuuti J, Kolh P, Lancellotti P, Linhart A, Nihoyannopoulos P, Piepoli MF, Ponikowski P, Sirnes PA, Tamargo JL, Tendera M, Torbicki A, Wijns W, Windecker S, Valgimigli M, Claeys MJ, Donner-Banzhoff N, Frank H, Funck-Brentano C, Gaemperli O, Gonzalez-Juanatey JR, Hamilos M, Husted S, James SK, Kervinen K, Kristensen SD, Maggioni A Pietro, Pries AR, Romeo F, Rydén L, Simoons ML, Steg PG, Timmis A, Yildirir A. 2013 ESC guidelines on the management of stable coronary artery disease. Eur Heart J 2013;34:2949-3003.

114. Roffi M, Patrono C, Collet J-P, Mueller C, Valgimigli M, Andreotti F, Bax JJ, Borger MA, Brotons C, Chew DP, Gencer B, Hasenfuss G, Kjeldsen K, Lancellotti P, Landmesser U, Mehilli J, Mukherjee D, Storey RF, Windecker S. 2015 ESC Guidelines for the management of acute coronary syndromes in patients presenting without persistent ST-segment elevation. Eur Heart J 2015; ehv320. 
115. Jolicœur EM, Dunning A, Castelvecchio S, Dabrowski R, Waclawiw MA, Petrie MC, Stewart R, Jhund PS, Desvigne-Nickens P, Panza JA, Bonow RO, Sun B, San TR, Al-Khalidi HR, Rouleau JL, Velazquez EJ, Cleland JGF. Importance of angina in patients with coronary disease, heart failure, and left ventricular systolic dysfunction: insights from STICH. J Am Coll Cardiol 2015;66:2092-2100.

116. Allman KC, Shaw LJ, Hachamovitch R, Udelson JE. Myocardial viability testing and impact of revascularization on prognosis in patients with coronary artery disease and left ventricular dysfunction: a meta-analysis. J Am Coll Cardiol 2002;39: $1151-1158$.

117. Ling LF, Marwick TH, Flores DR, Jaber WA, Brunken RC, Cerqueira MD, Hachamovitch R. Identification of therapeutic benefit from revascularization in patients with left ventricular systolic dysfunction inducible ischemia versus hibernating myocardium. Circ Cardiovasc Imaging 2013;6:363-372.

118. Bonow RO, Maurer G, Lee KL, Holly TA, Binkley PF, Desvigne-Nickens P, Drozdz J, Farsky PS, Feldman AM, Doenst T, Michler RE, Berman DS, Nicolau JC, Pellikka PA, Wrobel K, Alotti N, Asch FM, Favaloro LE, She L, Velazquez EJ, Jones RH, Panza JA. Myocardial viability and survival in ischemic left ventricular dysfunction. N Engl J Med 2011;364:1617-1625.

119. Corrà U, Piepoli MF, Adamopoulos S, Agostoni P, Coats AJS, Conraads V, Lambrinou E, Pieske B, Piotrowicz E, Schmid J-P, Seferović PM, Anker SD, Filippatos G, Ponikowski PP. Cardiopulmonary exercise testing in systolic heart failure in 2014: the evolving prognostic role: a position paper from the committee on exercise physiology and training of the heart failure association of the ESC. Eur J Heart Fail 2014;16:929-941.

120. Piepoli MF, Conraads V, Corrà U, Dickstein K, Francis DP, Jaarsma T, McMurray J, Pieske B, Piotrowicz E, Schmid J-P, Anker SD, Solal AC, Filippatos GS, Hoes AW, Gielen S, Giannuzzi P, Ponikowski PP. Exercise training in heart failure: from theory to practice. A consensus document of the Heart Failure Association and the European Association for Cardiovascular Prevention and Rehabilitation. Eur J Heart Fail 2011;13:347-357.

121. Volpicelli G, Elbarbary M, Blaivas M, Lichtenstein DA, Mathis G, Kirkpatrick AW, Melniker L, Gargani L, Noble VE, Via G, Dean A, Tsung JW, Soldati G, Copetti R, Bouhemad B, Reissig A, Agricola E, Rouby J-J, Arbelot C, Liteplo A, Sargsyan A, Silva F, Hoppmann R, Breitkreutz R, Seibel A, Neri L, Storti E, Petrovic T. International evidence-based recommendations for point-of-care lung ultrasound. Intensive Care Med 2012;38:577-591.

122. Roma-Rodrigues C, Fernandes AR. Genetics of hypertrophic cardiomyopathy: advances and pitfalls in molecular diagnosis and therapy. Appl Clin Genet 2014;7: 195-208.

123. McNally EM, Golbus JR, Puckelwartz MJ. Genetic mutations and mechanisms in dilated cardiomyopathy. J Clin Invest 2013;123:19-26.

124. Priori SG, Wilde AA, Horie M, Cho Y, Behr ER, Berul C, Blom N, Brugada J, Chiang CE, Huikuri $H$, Kannankeril P, Krahn A, Leenhardt A, Moss A, Schwartz PJ, Shimizu W, Tomaselli G, Tracy C. HRS/EHRA/APHRS expert consensus statement on the diagnosis and management of patients with inherited primary arrhythmia syndromes: document endorsed by HRS, EHRA, and APHRS in May 2013 and by ACCF, AHA, PACES, and AEPC in June 2013. Heart Rhythm 2013;10:1932-1963.

125. Arbustini E, Narula N, Tavazzi L, Serio A, Grasso M, Favalli V, Bellazzi R, Tajik JA, Bonow RD, Fuster V, Narula J. The MOGE(S) classification of cardiomyopathy for clinicians. J Am Coll Cardiol 2014;64:304-318.

126. Kostis JB, Davis BR, Cutler J, Grimm RH, Berge KG, Cohen JD, Lacy CR, Perry HM, Blaufox MD, Wassertheil-Smoller S, Black HR, Schron E, Berkson DM, Curb JD, Smith WM, McDonald R, Applegate WB, SHEP Cooperative Research Group. Prevention of heart failure by antihypertensive drug treatment in older persons with isolated systolic hypertension. JAMA 1997;278:212-216.

127. Beckett NS, Peters R, Fletcher AE, Staessen JA, Liu L, Dumitrascu D, Stoyanovsky V, Antikainen RL, Nikitin Y, Anderson C, Belhani A, Forette F, Rajkumar C, Thijs L, Banya W, Bulpitt C). Treatment of hypertension in patients 80 years of age or older. N Engl J Med 2008;358:1887-1898.

128. Sciarretta S, Palano F, Tocci G, Baldini R, Volpe M. Antihypertensive treatment and development of heart failure in hypertension. Arch Intern Med 2011;171:384-394.

129. Wright JT, Williamson JD, Whelton PK, Snyder JK, Sink KM, Rocco M V, Reboussin DM, Rahman M, Oparil S, Lewis CE, Kimmel PL, Johnson KC, Goff DC, Fine LJ, Cutler JA, Cushman WC, Cheung AK, Ambrosius WT, SPRINT Research Group. A randomized trial of intensive versus standard blood-pressure control. N Engl J Med 2015;373:2103-2116.

130. Zinman B, Wanner C, Lachin JM, Fitchett D, Bluhmki E, Hantel S, Mattheus M, Devins T, Johansen OE, Woerle HJ, Broedl UC, Inzucchi SE, EMPA-REG OUTCOME Investigators. Empagliflozin, cardiovascular outcomes, and mortality in type 2 diabetes. N Engl J Med 2015;373:2117-2128.

131. Suskin N, Sheth T, Negassa A, Yusuf S. Relationship of current and past smoking to mortality and morbidity in patients with left ventricular dysfunction. J Am Coll Cardiol 2001;37:1677-1682.
132. Dorans KS, Mostofsky E, Levitan EB, Håkansson N, Wolk A, Mittleman MA. Alcohol and incident heart failure among middle-aged and elderly men: the cohort of Swedish men. Circ Heart Fail 2015;8:422-427.

133. Goncalves A, Claggett B, Jhund PS, Rosamond W, Deswal A, Aguilar D, Shah AM, Cheng S, Solomon SD. Alcohol consumption and risk of heart failure: the Atherosclerosis Risk in Communities Study. Eur Heart J 2015;36:939-945.

134. Larsson SC, Orsini N, Wolk A. Alcohol consumption and risk of heart failure: a dose-response meta-analysis of prospective studies. Eur J Heart Fail 2015;17: 367-373.

135. Pandey A, Garg S, Khunger M, Darden D, Ayers C, Kumbhani DJ, Mayo HG, de Lemos JA, Berry JD. Dose-response relationship between physical activity and risk of heart failure: a meta-analysis. Circulation 2015;132:1786-1794.

136. Ledwidge M, Gallagher J, Conlon C, Tallon E, O'Connell E, Dawkins I, Watson C, O'Hanlon R, Bermingham M, Patle A, Badabhagni MR, Murtagh G, Voon V, Tilson L, Barry M, McDonald L, Maurer B, McDonald K. Natriuretic peptide-based screening and collaborative care for heart failure: the STOP-HF randomized trial. JAMA 2013;310:66-74.

137. Scirica BM, Morrow DA, Cannon CP, Ray KK, Sabatine MS, Jarolim P, Shui A, McCabe $\mathrm{CH}$, Braunwald $\mathrm{E}$. Intensive statin therapy and the risk of hospitalization for heart failure after an acute coronary syndrome in the PROVE IT-TIMI 22 study. J Am Coll Cardiol 2006;47:2326-2331.

138. Afilalo J, Majdan AA, Eisenberg MJ. Intensive statin therapy in acute coronary syndromes and stable coronary heart disease: a comparative meta-analysis of randomised controlled trials. Heart 2007;93:914-921.

139. Kjekshus J, Pedersen TR, Olsson AG, Faergeman O, Pyörälä K. The effects of simvastatin on the incidence of heart failure in patients with coronary heart disease. J Card Fail 1997;3:249-254.

140. Protection H, Collaborative S, Emberson JR, Ng LL, Armitage J, Bowman L, Parish S, Collins R. N-terminal pro-B-type natriuretic peptide, vascular disease risk, and cholesterol reduction among 20,536 patients in the MRC/BHF Heart Protection Study. J Am Coll Cardiol 2007;49:311-319.

141. Kenchaiah S, Evans JC, Levy D, Wilson PWF, Benjamin EJ, Larson MG, Kannel WB, Vasan RS. Obesity and the risk of heart failure. N Engl J Med 2002;347:305-313.

142. Dagenais GR, Pogue J, Fox K, Simoons ML, Yusuf S. Angiotensin-converting-enzyme inhibitors in stable vascular disease without left ventricular systolic dysfunction or heart failure: a combined analysis of three trials. Lancet 2006;368:581-588.

143. Huelsmann M, Neuhold S, Resl M, Strunk G, Brath H, Francesconi C, Adlbrecht C, Prager R, Luger A, Pacher R, Clodi M. PONTIAC (NT-proBNP selected prevention of cardiac events in a population of diabetic patients without a history of cardiac disease): a prospective randomized controlled trial. J Am Coll Cardiol 2013;62: $1365-1372$.

144. Jong P, Yusuf S, Rousseau MF, Ahn SA, Bangdiwala SI. Effect of enalapril on 12-year survival and life expectancy in patients with left ventricular systolic dysfunction: a follow-up study. Lancet 2003;361:1843-1848.

145. Pfeffer MA, Braunwald E, Moyé LA, Basta L, Brown EJ, Cuddy TE, Davis BR, Geltman EM, Goldman S, Flaker GC, Klein M, Lamas GA, Packer M, Rouleau JLJ, Rouleau JLJ, Rutherford J, Wertheimer JH, Hawkins CM. Effect of captopril on mortality and morbidity in patients with left ventricular dysfunction after myocardial infarction. N Engl J Med 1992;327:669-677.

146. Dargie HJ. Effect of carvedilol on outcome after myocardial infarction in patients with left-ventricular dysfunction: the CAPRICORN randomised trial. Lancet 2001; 357:1385-1390.

147. Montalescot G, Pitt B, Lopez de Sa E, Hamm CW, Flather M, Verheugt F, Shi H, Turgonyi E, Orri M, Vincent J, Zannad F, Noll G, Weir R, O'Neill B, Bohm M, Hillis WS, Grieve A, Rouleau J-L, Gerasimos F, Fitchett D, Lepage S, Madan M, Sussex B, Tremblay G, Welsh R, Wong G, Hutyra M, Kettner J, Ostadal P, Spinar J, Vojacek J, Barboteu M, Collet J-P, Coste P, Cottin Y, Ducos D, Galinier M, Teiger E, Zemour G, Bauersachs J, Hambrecht R, Hauf G, Heuer H, Mudra H, Munzel T, Steiner S, Strasser R, Sydow K, Tschope C, Wachter R, Werner N, Alexopoulos D, Babalis D, Pyrgakis V, Dezsi C, Lupkovics G, Polgar P, Tomcsanyi J, Herrman J, ten Berg JM, Gorny J, Kubica J, Lewczuk J, Zmuda W, Hranai M, Kovar F, Margoczy R, Micko K, Sumbal J, Genover XB, Ortiz AF, Sala MF, Garcia CG, Munoz CP, Rey Blas JR, Soriano FR, Adamson D, Alamgir F, Chauhan A, Lip G, Martin T, McCann G, Newby D, Smith D. Early eplerenone treatment in patients with acute ST-elevation myocardial infarction without heart failure: the Randomized Double-Blind Reminder Study. Eur Heart J 2014;35:2295-2302.

148. Pitt B, Remme W, Zannad F, Neaton J, Martinez F, Roniker B, Bittman R, Hurley S, Kleiman J, Gatlin M. Eplerenone, a selective aldosterone blocker, in patients with left ventricular dysfunction after myocardial infarction. N Engl J Med 2003;348: 1309-1321.

149. Moss AJ, Zareba W, Hall WJ, Klein H, Wilber DJ, Cannom DS, Daubert JP, Higgins SL, Brown MW, Andrews ML. Prophylactic implantation of a defibrillator 
in patients with myocardial infarction and reduced ejection fraction. N Engl / Med 2002;346:877-883.

150. Beckett NS, Peters R, Fletcher AE, Staessen JA, Liu L, Dumitrascu D, Stoyanovsky V, Antikainen RL, Nikitin Y, Anderson C, Belhani A, Forette F, Rajkumar C, Thijs L, Banya W, Bulpitt C]. Treatment of hypertension in patients 80 years of age or older. N Engl J Med 2008;358:1887-1898.

151. Sciarretta S, Palano F, Tocci G, Baldini R, Volpe M. Antihypertensive treatment and development of heart failure in hypertension: a Bayesian network meta-analysis of studies in patients with hypertension and high cardiovascular risk. Arch Intern Med 2011;171:384-394.

152. Preiss D, Campbell RT, Murray HM, Ford I, Packard CJ, Sattar N, Rahimi K, Colhoun HM, Waters DD, LaRosa JC, Amarenco P, Pedersen TR, Tikkanen MJ, Koren MJ, Poulter NR, Sever PS, Ridker PM, MacFadyen JG, Solomon SD, Davis BR, Simpson LM, Nakamura H, Mizuno K, Marfisi RM, Marchioli R, Tognoni G, Athyros VG, Ray KK, Gotto AM, Clearfield MB, Downs JR, McMurray J]. The effect of statin therapy on heart failure events: a collaborative meta-analysis of unpublished data from major randomized trials. Eur Heart J 2015;36:1536-1546.

153. Udell JA, Cavender MA, Bhatt DL, Chatterjee S, Farkouh ME, Scirica BM. Glucoselowering drugs or strategies and cardiovascular outcomes in patients with or at risk for type 2 diabetes: a meta-analysis of randomised controlled trials. Lancet Diabetes Endocrinol 2015;3:356-366.

154. Padwal R, McAlister Fa, McMurray JJ V, Cowie MR, Rich M, Pocock S, Swedberg K, Maggioni A, Gamble G, Ariti C, Earle N, Whalley G, Poppe KK, Doughty RN, Bayes-Genis A. The obesity paradox in heart failure patients with preserved versus reduced ejection fraction: a meta-analysis of individual patient data. Int $J$ Obes 2014;38:1110-1114.

155. Held C, Gerstein HC, Yusuf S, Zhao F, Hilbrich L, Anderson C, Sleight P, Teo K, ONTARGET/TRANSCEND Investigators. Glucose levels predict hospitalization for congestive heart failure in patients at high cardiovascular risk. Circulation 2007; 115:1371-1375.

156. Desai AS, Fang JC, Maisel WH, Baughman KL. Implantable defibrillators for the prevention of mortality in patients with nonischemic cardiomyopathy: a meta-analysis of randomized controlled trials. JAMA 2004;292:2874-2879.

157. Kadish A, Dyer A, Daubert JP, Quigg R, Estes NAM, Anderson KP, Calkins H, Hoch D, Goldberger J, Shalaby A, Sanders WE, Schaechter A, Levine JH, Defibrillators in Non-Ischemic Cardiomyopathy Treatment Evaluation (DEFINITE) Investigators. Prophylactic defibrillator implantation in patients with nonischemic dilated cardiomyopathy. N Engl J Med 2004;350:2151-2158.

158. Hohnloser SH, Kuck KH, Dorian P, Roberts RS, Hampton JR, Hatala R, Fain E, Gent M, Connolly SJ. Prophylactic use of an implantable cardioverter-defibrillator after acute myocardial infarction. N Engl J Med 2004;351:2481-2488.

159. Stewart S, Jenkins A, Buchan S, McGuire A, Capewell S, McMurray JJJV. The current cost of heart failure to the National Health Service in the UK. Eur J Heart Fail 2002;4:361-371.

160. Gheorghiade M, Shah AN, Vaduganathan M, Butler J, Bonow RO, Rosano GMC, Taylor S, Kupfer S, Misselwitz F, Sharma A, Fonarow GC. Recognizing hospitalized heart failure as an entity and developing new therapies to improve outcomes: academics', clinicians', industry's, regulators', and payers' perspectives. Heart Fail Clin 2013;9:285-290, v-vi.

161. Ambrosy AP, Fonarow GC, Butler J, Chioncel O, Greene SJ, Vaduganathan M, Nodari S, Lam CSP, Sato N, Shah AN, Gheorghiade M. The global health and economic burden of hospitalizations for heart failure. J Am Coll Cardiol 2014;63: 1123-1133.

162. McMurray JJ, Packer M, Desai AS, Gong J, Lefkowitz MP, Rizkala AR, Rouleau JL, Shi VC, Solomon SD, Swedberg K, Zile MR, PARADIGM-HF Investigators and Committees. Angiotensin-neprilysin inhibition versus enalapril in heart failure. $N$ Engl J Med 2014;371:993-1004.

163. Garg R, Yusuf S. Overview of randomized trials of angiotensin-converting enzyme inhibitors on mortality and morbidity in patients with heart failure. JAMA 1995; 273:1450-1456.

164. Packer M, Poole-Wilson PA, Armstrong PW, Cleland JGF, Horowitz JD, Massie BM, Ryden L, Thygesen K, Uretsky BF, Rydén L, Thygesen K, Uretsky BF, ATLAS Study Group. Comparative effects of low and high doses of the angiotensin-converting enzyme inhibitor, lisinopril, on morbidity and mortality in chronic heart failure. Circulation 1999;100:2312-2318.

165. SOLVD Investigattors. Effect of enalapril on survival in patients with reduced left ventricular ejection fractions and congestive heart failure. N Engl J Med 1991;325: 293-302.

166. Maggioni AP, Anker SD, Dahlström U, Filippatos G, Ponikowski P, Zannad F, Amir O, Chioncel O, Leiro MC, Drozdz J, Erglis A, Fazlibegovic E, Fonseca C, Fruhwald F, Gatzov P, Goncalvesova E, Hassanein M, Hradec J, Kavoliuniene A, Lainscak M, Logeart D, Merkely B, Metra M, Persson H, Seferovic P, Temizhan A, Tousoulis D, Tavazzi L. Are hospitalized or ambulatory patients with heart failure treated in accordance with European Society of Cardiology guidelines? Evidence from 12440 patients of the ESC Heart Failure Long-Term Registry. Eur J Heart Fail 2013;15:1173-1184.

167. Hjalmarson A, Goldstein S, Fagerberg B, Wedel H, Waagstein F, Kjekshus J, Wikstrand J, ElAllaf D, Vítovec J, Aldershvile J, Halinen M, Dietz R, Neuhaus KL, Jánosi A, Thorgeirsson G, Dunselman PH, Gullestad L, Kuch J, Herlitz J, Rickenbacher P, Ball S, Gottlieb S, Deedwania P. MERIT-HF Study Group. Effects of controlled-release metoprolol on total mortality, hospitalizations, and wellbeing in patients with heart failure: the Metoprolol CR/XL Randomized Intervention Trial in congestive Heart Failure (MERIT-HF). JAMA 2000;283:1295-1302.

168. Packer M, Coats AJ, Fowler MB, Katus HA, Krum H, Mohacsi P, Rouleau JL, Tendera M, Castaigne A, Roecker EB, Schultz MK, DeMets DL. Effect of carvedilol on survival in severe chronic heart failure. N Engl J Med 2001;344:1651-1658.

169. Packer M, Bristow MR, Cohn JN, Colucci WS, Fowler MB, Gilbert EM, Shusterman $\mathrm{NH}$. The effect of carvedilol on morbidity and mortality in patients with chronic heart failure. N Engl J Med 1996;334:1349-1355.

170. Effect of metoprolol CR/XL in chronic heart failure: Metoprolol CR/XL Randomised Intervention Trial in Congestive Heart Failure (MERIT-HF). Lancet 1999; 353:2001-2007.

171. Packer M. Effect of carvedilol on the morbidity of patients with severe chronic heart failure: results of the Carvedilol Prospective Randomized Cumulative Survival (COPERNICUS) Study. Circulation 2002;106:2194-2199.

172. CIBIS-II Investigators and Committees. The Cardiac Insufficiency Bisoprolol Study II (CIBIS-II): a randomised trial. Lancet 1999;353:9-13.

173. Flather MD, Shibata MC, Coats AJS, Van Veldhuisen DJ, Parkhomenko A, Borbola J, Cohen-Solal A, Dumitrascu D, Ferrari R, Lechat P, Soler-Soler J, Tavazzi L, Spinarova L, Toman J, Böhm M, Anker SD, Thompson SG, Poole-Wilson PA, SENIORS Investigators. Randomized trial to determine the effect of nebivolol on mortality and cardiovascular hospital admission in elderly patients with heart failure (SENIORS). Eur Heart J 2005;26:215-225.

174. Pitt B, Zannad F, Remme WJ, Cody R, Castaigne A, Perez A, Palensky J, Wittes J. The effect of spironolactone on morbidity and mortality in patients with severe heart failure. N Engl J Med 1999;341:709-717.

175. Zannad F, McMurray JJV, Krum H, Van Veldhuisen DJ, Swedberg K, Shi H, Vincent J, Pocock SJ, Pitt B. Eplerenone in patients with systolic heart failure and mild symptoms. N Engl I Med 2011;364:11-21.

176. Willenheimer R, van Veldhuisen DJ, Silke B, Erdmann E, Follath F, Krum H, Ponikowski P, Skene A, van de Ven L, Verkenne P, Lechat P, CIBIS III Investigators. Effect on survival and hospitalization of initiating treatment for chronic heart failure with bisoprolol followed by enalapril, as compared with the opposite sequence: results of the randomized Cardiac Insufficiency Bisoprolol Study (CIBIS) III. Circulation 2005;112:2426-2435.

177. Kotecha D, Holmes J, Krum H, Altman DG, Manzano L, Cleland JGF, Lip GYH, Coats AJS, Andersson B, Kirchhof P, von Lueder TG, Wedel H, Rosano G, Shibata MC, Rigby A, Flather MD. Efficacy of $\beta$ blockers in patients with heart failure plus atrial fibrillation: an individual-patient data meta-analysis. Lancet 2014 384:2235-2243.

178. Faris RF, Flather M, Purcell H, Poole-Wilson PA, Coats AJ. Diuretics for heart failure. Cochrane Database Syst Rev 2012;2:CD003838.

179. Faris R, Flather M, Purcell H, Henein M, Poole-Wilson P, Coats A. Current evidence supporting the role of diuretics in heart failure: a meta analysis of randomised controlled trials. Int J Cardiol 2002;82:149-158.

180. Swedberg K, Komajda M, Böhm M, Borer JS, Ford I, Dubost-Brama A, Lerebours G, Tavazzi L. Ivabradine and outcomes in chronic heart failure (SHIFT): a randomised placebo-controlled study. Lancet 2010;376:875-885.

181. Swedberg K, Komajda M, Böhm M, Borer J, Robertson M, Tavazzi L, Ford I. Effects on outcomes of heart rate reduction by ivabradine in patients with congestive heart failure: is there an influence of beta-blocker dose?: findings from the SHIFT (Systolic Heart failure treatment with the I(f) inhibitor ivabradine Trial) study. J Am Coll Cardiol 2012;59:1938-1945.

182. Granger CB, McMurray JJV, Yusuf S, Held P, Michelson EL, Olofsson B, Ostergren J, Pfeffer MA, Swedberg K. Effects of candesartan in patients with chronic heart failure and reduced left-ventricular systolic function intolerant to angiotensin-converting-enzyme inhibitors: the CHARM-Alternative trial. Lancet 2003;362:772-776

183. Taylor AL, Ziesche S, Yancy C, Carson P, D'Agostino R, Ferdinand K, Taylor M, Adams K, Sabolinski M, Worcel M, Cohn JN. Combination of isosorbide dinitrate and hydralazine in blacks with heart failure. N Engl J Med 2004;351:2049-2057.

184. Cohn JN, Archibald DG, Ziesche S, Franciosa JA, Harston WE, Tristani FE, Dunkman WB, Jacobs W, Francis GS, Flohr KH, Goldman S, Cobb FR, Shah PM, Saunders R, Fletcher RD, Loeb HS, Hughes VC, Baker B. Effect of vasodilator therapy on mortality in chronic congestive heart failure. N EnglJ Med 1986; 314:1547-1552.

185. Digitalis Investigation Group. The effect of digoxin on mortality and morbidity in patients with heart failure. N Engl J Med 1997;336:525-533. 
186. Tavazzi L, Maggioni AP, Marchioli R, Barlera S, Franzosi MG, Latini R, Lucci D, Nicolosi GL, Porcu M, Tognoni G. Effect of n-3 polyunsaturated fatty acids in patients with chronic heart failure (the GISSI-HF trial): a randomised, double-blind, placebo-controlled trial. Lancet 2008;372:1223-1230.

187. King JB, Bress AP, Reese AD, Munger MA. Neprilysin inhibition in heart failure with reduced ejection fraction: a clinical review. Pharmacother J Hum Pharmacol Drug Ther 2015;35:823-837.

188. Mangiafico S, Costello-Boerrigter LC, Andersen IA, Cataliotti A, Burnett JC. Neutral endopeptidase inhibition and the natriuretic peptide system: an evolving strategy in cardiovascular therapeutics. Eur Heart / 2013;34:886-893.

189. Vodovar N, Paquet C, Mebazaa A, Launay J-M, Hugon J, Cohen-Solal A. Neprilysin, cardiovascular, and Alzheimer's diseases: the therapeutic split? Eur Heart J 2015;36:902-905.

190. Yasojima K, McGeer EG, McGeer PL. Relationship between beta amyloid peptide generating molecules and neprilysin in Alzheimer disease and normal brain. Brain Res 2001;919:115-121.

191. Vepsalainen S, Helisalmi S, Mannermaa A, Pirttila T, Soininen H, Hiltunen M, Vepsäläinen S, Helisalmi S, Mannermaa A, Pirttilä T, Soininen H, Hiltunen M. Combined risk effects of IDE and NEP gene variants on Alzheimer disease. J Neurol Neurosurg Psychiatry 2009;80:1268-1270.

192. Langenickel TH, Tsubouchi C, Ayalasomayajula S, Pal P, Valentin M-A, Hinder M, Jhee S, Gevorkyan H, Rajman I. The effect of LCZ696 on amyloid- $\beta$ concentrations in cerebrospinal fluid in healthy subjects. Br J Clin Pharmacol 2015 Dec 12. doi:10.1111/bcp.12861 [Epub ahead of print]

193. Böhm M, Borer J, Ford I, Gonzalez-Juanatey JR, Komajda M, Lopez-Sendon J, Reil J-C, Swedberg K, Tavazzi L. Heart rate at baseline influences the effect of ivabradine on cardiovascular outcomes in chronic heart failure: analysis from the SHIFT study. Clin Res Cardiol 2013;102:11-22.

194. Cohn JN, Tognoni G. A randomized trial of the angiotensin-receptor blocker valsartan in chronic heart failure. N Engl J Med 2001;345:1667-1675.

195. Ouyang A-J, Lv Y-N, Zhong H-L, Wen J-H, Wei X-H, Peng H-W, Zhou J, Liu L-L. Meta-analysis of digoxin use and risk of mortality in patients with atrial fibrillation. Am J Cardiol 2015;115:901-906.

196. Vamos M, Erath JW, Hohnloser SH. Digoxin-associated mortality: a systematic review and meta-analysis of the literature. Eur Heart J 2015;36:1831-1838.

197. Ziff OJ, Lane DA, Samra M, Griffith M, Kirchhof P, Lip GYH, Steeds RP, Townend J, Kotecha D. Safety and efficacy of digoxin: systematic review and meta-analysis of observational and controlled trial data. BMJ 2015;351:h4451.

198. Van Gelder IC, Groenveld HF, Crijns HJGM, Tuininga YS, Tijssen JGP, Alings AM, Hillege HL, Bergsma-Kadijk JA, Cornel JH, Kamp O, Tukkie R, Bosker HA, Van Veldhuisen DJ, Van den Berg MP, RACE II Investigators. Lenient versus strict rate control in patients with atrial fibrillation. N Engl J Med 2010;362:1363-1373.

199. Bavishi C, Khan AR, Ather S. Digoxin in patients with atrial fibrillation and heart failure: a meta-analysis. Int J Cardiol 2015;188:99-101.

200. Freeman JV, Reynolds K, Fang M, Udaltsova N, Steimle A, Pomernacki NK, Borowsky LH, Harrison TN, Singer DE, Go AS. Digoxin and risk of death in adults with atrial fibrillation: the ATRIA-CVRN Study. Circ Arrhythmia Electrophysiol 2015; 8:49-58.

201. Washam JB, Stevens SR, Lokhnygina Y, Halperin JL, Breithardt G, Singer DE, Mahaffey KW, Hankey GJ, Berkowitz SD, Nessel CC, Fox KAA, Califf RM, Piccini JP, Patel MR. Digoxin use in patients with atrial fibrillation and adverse cardiovascular outcomes: a retrospective analysis of the Rivaroxaban Once Daily Oral Direct Factor Xa Inhibition Compared with Vitamin K Antagonism for Prevention of Stroke and Embolism Trial in Atrial Fibrillation (ROCKET AF). Lancet 2015;385:2363-2370.

202. Mulder BA, Van Veldhuisen DJ, Crijns HJGM, Tijssen JGP, Hillege HL, Alings M, Rienstra M, Groenveld HF, Van den Berg MP, Van Gelder IC, RACE II investigators. Lenient vs. strict rate control in patients with atrial fibrillation and heart failure: a post-hoc analysis of the RACE II study. Eur J Heart Fail 2013;15:1311-1318.

203. Lucas M, Kimmig M, Karalis G. Do omega-3 polyunsaturated fatty acids prevent cardiovascular disease? A review of the randomized clinical trials. Lipid Insights 2013;6:13-20.

204. Reiner Z, Catapano AL, De Backer G, Graham I, Taskinen M-R, Wiklund O, Agewall S, Alegria E, Chapman MJ, Durrington P, Erdine S, Halcox J, Hobbs R, Kjekshus J, Filardi PP, Riccardi G, Storey RF, Wood D, Bax J, Vahanian A, Auricchio A, Baumgartner $\mathrm{H}$, Ceconi C, Dean V, Deaton C, Fagard R, Filippatos G, Funck-Brentano C, Hasdai D, Hobbs R, Hoes A, Kearney P, Knuuti J, Kolh P, McDonagh T, Moulin C, Poldermans D, Popescu BA, Reiner Z, Sechtem U, Sirnes PA, Tendera M, Torbicki A, Vardas P, Widimsky P, Windecker S, Reviewers: D, Funck-Brentano C, Poldermans D, Berkenboom G, De Graaf J, Descamps O, Gotcheva N, Griffith K, Guida GF, Gulec S, Henkin Y, Huber K, Kesaniemi YA, Lekakis J, Manolis AJ, Marques-Vidal P, Masana L, McMurray J, Mendes M, Pagava Z, Pedersen T, Prescott E, Rato Q, Rosano G, Sans S, Stalenhoef A, Tokgozoglu L, Viigimaa M, Wittekoek ME, Zamorano JL. ESC/EAS Guidelines for the management of dyslipidaemias: the Task Force for the management of dyslipidaemias of the European Society of Cardiology (ESC) and the European Atherosclerosis Society (EAS). Eur Heart J 2011;32:1769-1818.

205. Kjekshus J, Apetrei E, Barrios V, Böhm M, Cleland JGF, Cornel JH, Dunselman P, Fonseca C, Goudev A, Grande P, Gullestad L, Hjalmarson Å, Hradec J, Jánosi A, Kamenský G, Komajda M, Korewicki J, Kuusi T, Mach F, Mareev V, McMurray JJV, Ranjith N, Schaufelberger M, Vanhaecke J, van Veldhuisen DJ, Waagstein F, Wedel $\mathrm{H}$, Wikstrand J. Rosuvastatin in older patients with systolic heart failure. N Engl J Med 2007;357:2248-2261.

206. Homma S, Thompson JLP, Pullicino PM, Levin B, Freudenberger RS, Teerlink JR, Ammon SE, Graham S, Sacco RL, Mann DL, Mohr JP, Massie BM, Labovitz AJ, Anker SD, Lok DJ, Ponikowski P, Estol CJ, Lip GYH, Di Tullio MR, Sanford AR, Mejia V, Gabriel AP, del Valle ML, Buchsbaum R, Shunichi H, Thompson JLP, Pullicino PM, Levin B, Freudenberger RS, Teerlink JR, Ammon SE, Graham S, Sacco RL, Mann DL, Mohr JP, Massie BM, Labovitz AJ, Lip GYH, Tullio MR, Di, Sanford AR, Mejia V, Gabriel AP, Valle ML, Buchsbaum R, Investigators W. Warfarin and aspirin in patients with heart failure and sinus rhythm. N EnglJ Med 2012; 366:1859-1869.

207. Lip GYH, Ponikowski P, Andreotti F, Anker SD, Filippatos G, Homma S, Morais J, Pullicino P, Rasmussen LH, Marin F, Lane DA. Thrombo-embolism and antithrombotic therapy for heart failure in sinus rhythm. A joint consensus document from the ESC Heart Failure Association and the ESC Working Group on Thrombosis. Eur J Heart Fail 2012;14:681-695.

208. Gheorghiade M, Böhm M, Greene SJ, Fonarow GC, Lewis EF, Zannad F, Solomon SD, Baschiera F, Botha J, Hua TA, Gimpelewicz CR, Jaumont X, Lesogor A, Maggioni AP, ASTRONAUT Investigators and Coordinators. Effect of aliskiren on postdischarge mortality and heart failure readmissions among patients hospitalized for heart failure: the ASTRONAUT randomized trial. JAMA 2013;309:1125-1135.

209. Hernandez AV, Usmani A, Rajamanickam A, Moheet A. Thiazolidinediones and risk of heart failure in patients with or at high risk of type 2 diabetes mellitus: a meta-analysis and meta-regression analysis of placebo-controlled randomized clinical trials. Am J Cardiovasc Drugs 2011;11:115-128.

210. Komajda M, McMurray JJV, Beck-Nielsen H, Gomis R, Hanefeld M, Pocock SJ, Curtis PS, Jones NP, Home PD. Heart failure events with rosiglitazone in type 2 diabetes: data from the RECORD clinical trial. Eur Heart J 2010;31:824-831.

211. Mamdani M, Juurlink DN, Lee DS, Rochon PA, Kopp A, Naglie G, Austin PC, Laupacis A, Stukel TA. Cyclo-oxygenase-2 inhibitors versus non-selective nonsteroidal anti-inflammatory drugs and congestive heart failure outcomes in elderly patients: a population-based cohort study. Lancet 2004;363:1751-1756.

212. Huerta C, Varas-Lorenzo C, Castellsague J, García Rodríguez LA. Non-steroidal anti-inflammatory drugs and risk of first hospital admission for heart failure in the general population. Heart 2006;92:1610-1615.

213. Scott $P$ a, Kingsley GH, Scott DL. Non-steroidal anti-inflammatory drugs and cardiac failure: meta-analyses of observational studies and randomised controlled trials. Eur J Heart Fail 2008;10:1102-1107.

214. Goldstein RE, Boccuzzi SJ, Cruess D, Nattel S. Diltiazem increases late-onset congestive heart failure in postinfarction patients with early reduction in ejection fraction. The Adverse Experience Committee and the Multicenter Diltiazem Postinfarction Research Group. Circulation 1991;83:52-60.

215. Packer M, O'Connor CM, Ghali JK, Pressler ML, Carson PE, Belkin RN, Miller AB, Neuberg GW, Frid D, Wertheimer JH, Cropp AB, DeMets DL, Prospective Randomized Amlodipine Survival Evaluation Study Group. Effect of amlodipine on morbidity and mortality in severe chronic heart failure. N Engl J Med 1996;335: 1107-1114.

216. Cohn JN, Ziesche S, Smith R, Anand I, Dunkman WB, Loeb H, Cintron G, Boden W, Baruch L, Rochin P, Loss L. Effect of the calcium antagonist felodipine as supplementary vasodilator therapy in patients with chronic heart failure treated with enalapril: V-HeFT III. Vasodilator-Heart Failure Trial (V-HeFT) Study Group. Circulation 1997;96:856-863.

217. Abraham WT, Zile MR, Weaver FA, Butter C, Ducharme A, Halbach M, Klug D, Lovett EG, Muller-Ehmsen J, Schafer JE, Senni M, Swarup V, Wachter R, Little WC. Baroreflex activation therapy for the treatment of heart failure with a reduced ejection fraction. JACC Heart Fail 2015;3:487-496.

218. Zannad F, De Ferrari GM, Tuinenburg AE, Wright D, Brugada J, Butter C, Klein H, Stolen C, Meyer S, Stein KM, Ramuzat A, Schubert B, Daum D, Neuzil P, Botman C, Castel MA, D’Onofrio A, Solomon SD, Wold N, Ruble SB. Chronic vagal stimulation for the treatment of low ejection fraction heart failure: results of the NEural Cardiac TherApy foR Heart Failure (NECTAR-HF) randomized controlled trial. Eur Heart J 2015;36:425-433.

219. Ponikowski P, Javaheri S, Michalkiewicz D, Bart BA, Czarnecka D, Jastrzebski M, Kusiak A, Augostini R, Jagielski D, Witkowski T, Khayat RN, Oldenburg O, Gutleben K-J, Bitter T, Karim R, Iber C, Hasan A, Hibler K, Germany R, Abraham WT. Transvenous phrenic nerve stimulation for the treatment of central sleep apnoea in heart failure. Eur Heart J 2012;33:889-894. 
220. Abraham WT, Jagielski D, Oldenburg O, Augostini R, Krueger S, Kolodziej A, Gutleben K-J, Khayat R, Merliss A, Harsch MR, Holcomb RG, Javaheri S, Ponikowski P. Phrenic nerve stimulation for the treatment of central sleep apnea. JACC Heart Fail 2015;3:360-369.

221. Kadish A, Nademanee K, Volosin K, Krueger S, Neelagaru S, Raval N, Obel O, Weiner S, Wish M, Carson P, Ellenbogen K, Bourge R, Parides M, Chiacchierini RP, Goldsmith R, Goldstein S, Mika Y, Burkhoff D, Abraham WT. A randomized controlled trial evaluating the safety and efficacy of cardiac contractility modulation in advanced heart failure. Am Heart J 2011;161:329-337.e2.

222. Borggrefe MM, Lawo T, Butter C, Schmidinger H, Lunati M, Pieske B, Misier AR, Curnis A, Böcker D, Remppis A, Kautzner J, Stühlinger M, Leclerq C, Táborský M, Frigerio M, Parides M, Burkhoff D, Hindricks G. Randomized, double blind study of non-excitatory, cardiac contractility modulation electrical impulses for symptomatic heart failure. Eur Heart J 2008;29:1019-1028.

223. Wyse DG, Friedman PL, Epstein AE. A comparison of antiarrhythmic-drug therapy with implantable defibrillators in patients resuscitated from near-fatal ventricular arrhythmias. The Antiarrhythmics versus Implantable Defibrillators (AVID) Investigators. N Engl J Med 1997;337:1576-1583.

224. Connolly SJ, Hallstrom AP, Cappato R, Schron EB, Kuck KH, Zipes DP, Greene HL, Boczor S, Domanski M, Follmann D, Gent M, Roberts RS. Meta-analysis of the implantable cardioverter defibrillator secondary prevention trials. AVID, CASH and CIDS studies. Antiarrhythmics vs Implantable Defibrillator study. Cardiac Arrest Study Hamburg. Canadian Implantable Defibrillator Study. Eur Heart J 2000;21:2071-2078.

225. Connolly SJ, Gent M, Roberts RS, Dorian P, Roy D, Sheldon RS, Mitchell LB, Green MS, Klein GJ, O'Brien B. Canadian implantable defibrillator study (CIDS) : a randomized trial of the implantable cardioverter defibrillator against amiodarone. Circulation 2000;101:1297-1302.

226. Kuck KH, Cappato R, Siebels J, Rüppel R. Randomized comparison of antiarrhythmic drug therapy with implantable defibrillators in patients resuscitated from cardiac arrest: the Cardiac Arrest Study Hamburg (CASH). Circulation 2000;102: 748-754.

227. Bardy GH, Lee KL, Mark DB, Poole JE, Packer DL, Boineau R, Domanski M, Troutman C, Anderson J, Johnson G, McNulty SE, Clapp-Channing N, Davidson-Ray LD, Fraulo ES, Fishbein DP, Luceri RM, Ip JH. Amiodarone or an implantable cardioverter-defibrillator for congestive heart failure. N Engl I Med 2005;352:225-237.

228. Steinbeck G, Andresen D, Seidl K, Brachmann J, Hoffmann E, Wojciechowski D, Kornacewicz-Jach Z, Sredniawa B, Lupkovics G, Hofgärtner F, Lubinski A, Rosenqvist M, Habets A, Wegscheider K, Senges J. Defibrillator implantation early after myocardial infarction. N Engl J Med 2009;361:1427-1436.

229. Sanders GD, Hlatky MA, Owens DK. Cost-effectiveness of implantable cardioverter-defibrillators. N Engl J Med 2005;353:1471-1480.

230. Steinberg BA, Al-Khatib SM, Edwards R, Han J, Bardy GH, Bigger JT, Buxton AE, Moss AJ, Lee KL, Steinman R, Dorian P, Hallstrom A, Cappato R, Kadish AH, Kudenchuk PJ, Mark DB, Inoue LYT, Sanders GD. Outcomes of implantable cardioverter-defibrillator use in patients with comorbidities: results from a combined analysis of 4 randomized clinical trials. JACC Heart Fail 2014;2:623-629.

231. Raphael CE, Finegold JA, Barron AJ, Whinnett ZI, Mayet J, Linde C, Cleland JGF, Levy WC, Francis DP. The effect of duration of follow-up and presence of competing risk on lifespan-gain from implantable cardioverter defibrillator therapy: who benefits the most? Eur Heart J 2015;36:1676-1688.

232. Miller RJH, Howlett JG, Exner DV, Campbell PM, Grant ADM, Wilton SB. Baseline functional class and therapeutic efficacy of common heart failure interventions: a systematic review and meta-analysis. Can / Cardiol 2015;31:792-799.

233. Hess PL, Al-Khatib SM, Han JY, Edwards R, Bardy GH, Bigger JT, Buxton A, Cappato R, Dorian P, Hallstrom A, Kadish AH, Kudenchuk PJ, Lee KL, Mark DB, Moss AJ, Steinman R, Inoue LYT, Sanders G. Survival benefit of the primary prevention implantable cardioverter-defibrillator among older patients: does age matter? An analysis of pooled data from 5 clinical trials. Circ Cardiovasc Qual Outcomes 2015;8:179-186.

234. Merchant FM, Jones P, Wehrenberg S, Lloyd MS, Saxon LA. Incidence of defibrillator shocks after elective generator exchange following uneventful first battery life. J Am Heart Assoc 2014;3:e001289.

235. Yap S-C, Schaer BA, Bhagwandien RE, Kuhne M, Dabiri Abkenari L, Osswald S, Szili-Torok T, Sticherling C, Theuns DA. Evaluation of the need of elective implantable cardioverter-defibrillator generator replacement in primary prevention patients without prior appropriate ICD therapy. Heart 2014;100:1188-1192.

236. Kini V, Soufi MK, Deo R, Epstein AE, Bala R, Riley M, Groeneveld PW, Shalaby A, Dixit S. Appropriateness of primary prevention implantable cardioverterdefibrillators at the time of generator replacement: are indications still met? J Am Coll Cardiol 2014;63:2388-2394.

237. Erkapic D, Sperzel J, Stiller S, Meltendorf U, Mermi J, Wegscheider K, Hügl B. Long-term benefit of implantable cardioverter/defibrillator therapy after elective device replacement: Results of the INcidence free SUrvival after ICD
REplacement (INSURE) trial-a prospective multicentre study. Eur Heart J 2013;34:130-137.

238. Alsheikh-Ali AA, Homer M, Maddukuri PV, Kalsmith B, Estes NAM, Link MS. Time-dependence of appropriate implantable defibrillator therapy in patients with ischemic cardiomyopathy. J Cardiovasc Electrophysiol 2008;19:784-789.

239. Opreanu M, Wan C, Singh V, Salehi N, Ahmad J, Szymkiewicz SJ, Thakur RK. Wearable cardioverter-defibrillator as a bridge to cardiac transplantation: a national database analysis. J Heart Lung Transplant 2015;34:1305-1309.

240. Zishiri ET, Williams S, Cronin EM, Blackstone EH, Ellis SG, Roselli EE, Smedira NG, Gillinov AM, Glad JA, Tchou PJ, Szymkiewicz SJ, Chung MK. Early risk of mortality after coronary artery revascularization in patients with left ventricular dysfunction and potential role of the wearable cardioverter defibrillator. Circ Arrhythm Electrophysiol 2013;6:117-128.

241. Chung MK, Szymkiewicz SJ, Shao M, Zishiri E, Niebauer MJ, Lindsay BD, Tchou PJ. Aggregate national experience with the wearable cardioverter-defibrillator. J Am Coll Cardiol 2010;56:194-203.

242. Effect of prophylactic amiodarone on mortality after acute myocardial infarction and in congestive heart failure: meta-analysis of individual data from 6500 patients in randomised trials. Amiodarone Trials Meta-Analysis Investigators. Lancet 1997; 350:1417-1424.

243. Andrey JL, Gomez-Soto FM, Romero SP, Escobar MA, García-Egido AA, Garcia-Arjona R, Gomez F, GAMIC (Grupo para Atencion Medica Integrada de Cadiz). Mortality of newly diagnosed heart failure treated with amiodarone: a propensity-matched study. Int J Cardiol 2011;151:175-181.

244. Torp-Pedersen C, Metra M, Spark P, Lukas MA, Moullet C, Scherhag A, Komajda M, Cleland JGF, Remme W, Di Lenarda A, Swedberg K, Poole-Wilson PA. The safety of amiodarone in patients with heart failure. J Card Fail 2007;13:340-345.

245. Piepoli M, Villani GQ, Ponikowski P, Wright A, Flather MD, Coats AJ. Overview and meta-analysis of randomised trials of amiodarone in chronic heart failure. Int J Cardiol 1998;66:1-10.

246. Chatterjee S, Ghosh J, Lichstein E, Aikat S, Mukherjee D. Meta-analysis of cardiovascular outcomes with dronedarone in patients with atrial fibrillation or heart failure. Am J Cardiol 2012;110:607-613.

247. Køber L, Torp-Pedersen C, McMurray JJV, Gøtzsche O, Lévy S, Crijns H, Amlie J, Carlsen J. Increased mortality after dronedarone therapy for severe heart failure. N Engl J Med 2008;358:2678-2687.

248. Echt DS, Liebson PR, Mitchell LB, Peters RW, Obias-Manno D, Barker AH, Arensberg D, Baker A, Friedman L, Greene HL, Huther ML, Richardson DW. Mortality and morbidity in patients receiving encainide, flecainide, or placebo. The Cardiac Arrhythmia Suppression Trial. N Engl J Med 1991;324:781-788.

249. Theuns DAMJ, Smith T, Hunink MGM, Bardy GH, Jordaens L. Effectiveness of prophylactic implantation of cardioverter-defibrillators without cardiac resynchronization therapy in patients with ischaemic or non-ischaemic heart disease: a systematic review and meta-analysis. Europace 2010;12:1564-1570.

250. Cook NR, Ridker PM. Advances in measuring the effect of individual predictors of cardiovascular risk: the role of reclassification measures. Ann Intern Med 2009;150: 795-802.

251. Moss AJ, Hall WJ, Cannom DS, Daubert JP, Higgins SL, Klein H, Levine JH, Saksena S, Waldo AL, Wilber D, Brown MW, Heo M. Improved survival with an implanted defibrillator in patients with coronary disease at high risk for ventricular arrhythmia. Multicenter Automatic Defibrillator Implantation Trial Investigators. N Engl J Med 1996;335:1933-1940.

252. Moss AJ, Schuger C, Beck CA, Brown MW, Cannom DS, Daubert JP, Estes NAM, Greenberg H, Hall WJ, Huang DT, Kautzner J, Klein H, McNitt S, Olshansky B, Shoda M, Wilber D, Zareba W. Reduction in inappropriate therapy and mortality through ICD programming. N Engl J Med 2012;367:2275-2283.

253. Gasparini M, Proclemer A, Klersy C, Kloppe A, Lunati M, Ferrer JBM, Hersi A, Gulaj M, Wijfels MCEF, Santi E, Manotta L, Arenal A. Effect of long-detection interval vs standard-detection interval for implantable cardioverter-defibrillators on antitachycardia pacing and shock delivery: the ADVANCE III randomized clinical trial. JAMA 2013;309:1903-1911.

254. Cleland JGF, Buga L. Device therapy: defibrillators-a shocking therapy for cardiomyopathy? Nat Rev Cardiol 2010;7:69-70.

255. Stewart GC, Weintraub JR, Pratibhu PP, Semigran MJ, Camuso JM, Brooks K, Tsang SW, Anello MS, Nguyen VT, Lewis EF, Nohria A, Desai AS, Givertz MM, Stevenson LW. Patient expectations from implantable defibrillators to prevent death in heart failure. J Card Fail 2010;16:106-113.

256. Bardy GH, Smith WM, Hood M a, Crozier IG, Melton IC, Jordaens L, Theuns D, Park RE, Wright DJ, Connelly DT, Fynn SP, Murgatroyd FD, Sperzel J, Neuzner J, Spitzer SG, Ardashev AV, Oduro A, Boersma L, Maass AH, Van Gelder IC, Wilde AA, van Dessel PF, Knops RE, Barr CS, Lupo P, Cappato R, Grace AA. An entirely subcutaneous implantable cardioverter-defibrillator. N Engl J Med 2010;363:36-44. 
257. Aziz S, Leon AR, El-Chami MF. The subcutaneous defibrillator. J Am Coll Cardiol 2014;63:1473-1479.

258. Olde Nordkamp LRA, Knops RE, Bardy GH, Blaauw Y, Boersma LVA, Bos JS, Delnoy PPHM, van Dessel PFHM, Driessen AHG, de Groot JR, Herrman JPR, Jordaens LJLM, Kooiman KM, Maass AH, Meine M, Mizusawa Y, Molhoek SG, van Opstal J, Tijssen JGP, Wilde AAM. Rationale and design of the PRAETORIAN trial: a Prospective, RAndomizEd comparison of subcuTaneOus and tRansvenous ImplANtable cardioverter-defibrillator therapy. Am Heart J 2012;163: 753-760.e2.

259. Burke MC, Gold MR, Knight BP, Barr CS, Theuns DAMJ, Boersma LVA, Knops RE, Weiss R, Leon AR, Herre JM, Husby M, Stein KM, Lambiase PD. Safety and efficacy of the totally subcutaneous implantable defibrillator: 2-year results from a pooled analysis of the IDE Study and EFFORTLESS Registry. J Am Coll Cardiol 2015;65: 1605-1615.

260. Priori SG, Blomström-Lundqvist C, Mazzanti A, Blom N, Borggrefe M, Camm J, Elliott PM, Fitzsimons D, Hatala R, Hindricks G, Kirchhof P, Kjeldsen K, Kuck K-H, Hernandez-Madrid A, Nikolaou N, Norekvål TM, Spaulding C, Van Veldhuisen DJ. 2015 ESC Guidelines for the management of patients with ventricular arrhythmias and the prevention of sudden cardiac death: the Task Force for the Management of Patients with Ventricular Arrhythmias and the Prevention of Sudden Cardiac Death of the Europe. Eur Heart J 2015;36:2793-2867.

261. Cazeau S, Leclercq C, Lavergne T, Walker S, Varma C, Linde C, Garrigue S, Kappenberger L, Haywood GA, Santini M, Bailleul C, Mabo P, Lazarus A, Ritter P, Levy T, McKenna W, Daubert J-C. Effects of multisite biventricular pacing in patients with heart failure and intraventricular conduction delay. N Engl I Med 2001;344:873-880

262. Cleland J, Daubert J, Erdmann E, Freemantle N, Gras D, Kappenberger L, Tavazzi $L$. The effect of cardiac resynchronization on morbidity and mortality in heart failure. N Engl J Med 2005;352:1539-1549.

263. Cleland JGF, Daubert J-C, Erdmann E, Freemantle N, Gras D, Kappenberger L, Tavazzi L. Longer-term effects of cardiac resynchronization therapy on mortality in heart failure [the CArdiac REsynchronization-Heart Failure (CARE-HF) trial extension phase]. Eur Heart J 2006;27:1928-1932.

264. Cleland JGF, Freemantle N, Erdmann E, Gras D, Kappenberger L, Tavazzi L, Daubert J-CC. Long-term mortality with cardiac resynchronization therapy in the Cardiac Resynchronization-Heart Failure (CARE-HF) trial. Eur J Heart Fail 2012;14:628-634.

265. Bristow MR, Saxon LA, Boehmer J, Krueger S, Kass DA, De Marco T, Carson P, DiCarlo L, DeMets D, White BG, DeVries DW, Feldman AM. Cardiac-resynchronization therapy with or without an implantable defibrillator in advanced chronic heart failure. N Engl J Med 2004;350:2140-2150.

266. Cleland JG, Abraham WT, Linde C, Gold MR, Young JB, Claude Daubert J, Sherfesee L, Wells GA, Tang ASL. An individual patient meta-analysis of five randomized trials assessing the effects of cardiac resynchronization therapy on morbidity and mortality in patients with symptomatic heart failure. Eur Heart J 2013; 34:3547-3556

267. Tang ASL, Wells GA, Talajic M, Arnold MO, Sheldon R, Connolly S, Hohnloser SH, Nichol G, Birnie DH, Sapp JL, Yee R, Healey JS, Rouleau JL. Cardiac-resynchronization therapy for mild-to-moderate heart failure. N Engl J Med 2010;363:2385-2395.

268. Moss AJ, Hall WJ, Cannom DS, Klein H, Brown MW, Daubert JP, Estes NAM, Foster E, Greenberg H, Higgins SL, Pfeffer MA, Solomon SD, Wilber D, Zareba W. Cardiac-resynchronization therapy for the prevention of heart-failure events. N Engl J Med 2009;361:1329-1338.

269. Goldenberg I, Kutyifa V, Klein HU, Cannom DS, Brown MW, Dan A, Daubert JP, Estes NAM, Foster E, Greenberg H, Kautzner J, Klempfner R, Kuniss M, Merkely B, Pfeffer MA, Quesada A, Viskin S, McNitt S, Polonsky B, Ghanem A, Solomon SD, Wilber D, Zareba W, Moss AJ. Survival with cardiac-resynchronization therapy in mild heart failure. N Engl J Med 2014;370:1694-1701.

270. Linde C, Abraham WT, Gold MR, St John Sutton M, Ghio S, Daubert C. Randomized trial of cardiac resynchronization in mildly symptomatic heart failure patients and in asymptomatic patients with left ventricular dysfunction and previous heart failure symptoms. J Am Coll Cardiol 2008;52:1834-1843.

271. Daubert C, Gold MR, Abraham WT, Ghio S, Hassager C, Goode G, Szili-Török T, Linde C, REVERSE Study Group. Prevention of disease progression by cardiac resynchronization therapy in patients with asymptomatic or mildly symptomatic left ventricular dysfunction: insights from the European cohort of the REVERSE trial. J Am Coll Cardiol 2009;54:1837-1846.

272. Linde C, Gold MR, Abraham WT, St John Sutton M, Ghio S, Cerkvenik J, Daubert C. Long-term impact of cardiac resynchronization therapy in mild heart failure: 5-year results from the REsynchronization reVErses Remodeling in Systolic left vEntricular dysfunction (REVERSE) study. Eur Heart J 2013;34:2592-2599.

273. Woods B, Hawkins N, Mealing S, Sutton A, Abraham WT, Beshai JF, Klein H, Sculpher M, Plummer CJ, Cowie MR. Individual patient data network meta-analysis of mortality effects of implantable cardiac devices. Heart 2015; 101:1800-1806.

274. Curtis AB, Worley SJ, Adamson PB, Chung ES, Niazi I, Sherfesee L, Shinn T, St John Sutton M. Biventricular pacing for atrioventricular block and systolic dysfunction. N Engl J Med 2013;368:1585-1593.

275. Brignole M, Botto G, Mont L, lacopino S, De Marchi G, Oddone D, Luzi M Tolosana JM, Navazio A, Menozzi C. Cardiac resynchronization therapy in patients undergoing atrioventricular junction ablation for permanent atrial fibrillation: a randomized trial. Eur Heart J 2011;32:2420-2429.

276. Leclercq C, Walker S, Linde C, Clementy J, Marshall AJ, Ritter P, Djiane P, Mabo P, Levy T, Gadler F, Bailleul C, Daubert J-C. Comparative effects of permanent biventricular and right-univentricular pacing in heart failure patients with chronic atrial fibrillation. Eur Heart J 2002;23:1780-1787.

277. Stavrakis S, Garabelli P, Reynolds DW. Cardiac resynchronization therapy after atrioventricular junction ablation for symptomatic atrial fibrillation: a meta-analysis. Europace 2012;14:1490-1497.

278. MacDonald MR, Connelly DT, Hawkins NM, Steedman T, Payne J, Shaw M, Denvir M, Bhagra S, Small S, Martin W, McMurray J], Petrie MC. Radiofrequency ablation for persistent atrial fibrillation in patients with advanced heart failure and severe left ventricular systolic dysfunction: a randomised controlled trial. Heart 2011;97:740-747.

279. Jones DG, Haldar SK, Hussain W, Sharma R, Francis DP, Rahman-Haley SL, McDonagh TA, Underwood SR, Markides V, Wong T. A randomized trial to assess catheter ablation versus rate control in the management of persistent atrial fibrillation in heart failure. J Am Coll Cardiol 2013;61:1894-1903.

280. Upadhyay GA, Choudhry NK, Auricchio A, Ruskin J, Singh JP. Cardiac resynchronization in patients with atrial fibrillation: a meta-analysis of prospective cohort studies. J Am Coll Cardiol 2008;52:1239-1246.

281. Gasparini M, Leclercq C, Lunati M, Landolina M, Auricchio A, Santini M, Boriani G, Lamp B, Proclemer A, Curnis A, Klersy C, Leyva F. Cardiac resynchronization therapy in patients with atrial fibrillation. The CERTIFY Study (Cardiac Resynchronization Therapy in Atrial Fibrillation Patients Multinational Registry). JACC Heart Fail 2013;1:500-507.

282. Gage RM, Burns KV, Bank AJ. Echocardiographic and clinical response to cardiac resynchronization therapy in heart failure patients with and without previous right ventricular pacing. Eur J Heart Fail 2014;16:1199-1205.

283. Ruschitzka F, Abraham WT, Singh JP, Bax JJ, Borer JS, Brugada J, Dickstein K, Ford I, Gorcsan J, Gras D, Krum H, Sogaard P, Holzmeister J. Cardiac-resynchronization therapy in heart failure with a narrow QRS complex. N Engl J Med 2013;369: 1395-1405.

284. Steffel J, Robertson M, Singh JP, Abraham WT, Bax JJ, Borer JS, Dickstein K, Ford I, Gorcsan J, Gras D, Krum H, Sogaard P, Holzmeister J, Brugada J, Ruschitzka F. The effect of QRS duration on cardiac resynchronization therapy in patients with a narrow QRS complex: a subgroup analysis of the EchoCRT trial. Eur Heart J 2015;36:1983-1989.

285. Zusterzeel R, Selzman KA, Sanders WE, Caños DA, O'Callaghan KM, Carpenter JL, Piña IL, Strauss DG. Cardiac resynchronization therapy in women: US Food and Drug Administration meta-analysis of patient-level data. JAMA Intern Med 2014;174:1340-1348.

286. Sohaib SMMA, Finegold JA, Nijjer SS, Hossain R, Linde C, Levy WC, Sutton R, Kanagaratnam P, Francis DP, Whinnett Zl. Opportunity to increase life span in narrow QRS cardiac resynchronization therapy recipients by deactivating ventricular pacing: evidence from randomized controlled trials. JACC Heart Fail 2015;3:327-336.

287. Cleland JGF, Calvert MJ, Verboven Y, Freemantle N. Effects of cardiac resynchronization therapy on long-term quality of life: an analysis from the Cardiac Resynchronisation-Heart Failure (CARE-HF) study. Am Heart J 2009;157: 457-466.

288. Cleland JGF, Mareev Y, Linde C. Reflections on EchoCRT: sound guidance on QRS duration and morphology for CRT? Eur Heart J 2015;36:1948-1951.

289. Linde C, Stahlberg M, Benson L, Braunschweig F, Edner M, Dahlstrom U, Alehagen $U$, Lund LH. Gender, underutilization of cardiac resynchronization therapy, and prognostic impact of QRS prolongation and left bundle branch block in heart failure. Europace 2015;17:424-431.

290. Doshi RN, Daoud EG, Fellows C, Turk K, Duran A, Hamdan MH, Pires LA, PAVE Study Group. Left ventricular-based cardiac stimulation post AV nodal ablation evaluation (the PAVE study). J Cardiovasc Electrophysiol 2005;16:1160-1165.

291. Funck RC, Mueller H-H, Lunati M, Piorkowski C, De Roy L, Paul V, Wittenberg M, Wuensch D, Blanc J-J. Characteristics of a large sample of candidates for permanent ventricular pacing included in the Biventricular Pacing for Atrio-ventricular Block to Prevent Cardiac Desynchronization Study (BioPace). Europace 2014; 16:354-362.

292. Chung ES, Leon AR, Tavazzi L, Sun J-P, Nihoyannopoulos P, Merlino J, Abraham WT, Ghio S, Leclercq C, Bax JJ, Yu C-M, Gorcsan J, StJohn Sutton M, 
De Sutter J, Murillo J. Results of the Predictors of Response to CRT (PROSPECT) Trial. Circulation 2008;117:2608-2616.

293. Wikstrom G, Blomström-Lundqvist C, Andren B, Lönnerholm S, Blomström P, Freemantle N, Remp T, Cleland JGF. The effects of aetiology on outcome in patients treated with cardiac resynchronization therapy in the CARE-HF trial. Eur Heart J 2009;30:782-788.

294. Khan FZ, Virdee MS, Palmer CR, Pugh PJ, O'Halloran D, Elsik M, Read P a, Begley D, Fynn SP, Dutka DP. Targeted left ventricular lead placement to guide cardiac resynchronization therapy: the TARGET study: a randomized, controlled trial. J Am Coll Cardiol 2012;59:1509-1518.

295. Saba S, Marek J, Schwartzman D, Jain S, Adelstein E, White P, Oyenuga OA, Onishi T, Soman P, Gorcsan J. Echocardiography-guided left ventricular lead placement for cardiac resynchronization therapy: results of the Speckle Tracking Assisted Resynchronization Therapy for Electrode Region Trial. Circ Heart Fail 2013;6:427-434.

296. Kosmala W, Marwick TH. Meta-analysis of effects of optimization of cardiac resynchronization therapy on left ventricular function, exercise capacity, and quality of life in patients with heart failure. Am J Cardiol 2014;113:988-994.

297. Whinnett ZI, Francis DP, Denis A, Willson K, Pascale P, Van Geldorp I, De Guillebon M, Ploux S, Ellenbogen K, Haïssaguerre M, Ritter P, Bordachar P. Comparison of different invasive hemodynamic methods for $A V$ delay optimization in patients with cardiac resynchronization therapy: Implications for clinical trial design and clinical practice. Int J Cardiol 2013;168:2228-2237.

298. Kuck K-H, Bordachar P, Borggrefe M, Boriani G, Burri H, Leyva F, Schauerte P, Theuns D, Thibault B, Kirchhof P, Hasenfuss G, Dickstein K, Leclercq C, Linde C, Tavazzi L, Ruschitzka F. New devices in heart failure: an European Heart Rhythm Association report: developed by the European Heart Rhythm Association; endorsed by the Heart Failure Association. Europace 2014;16:109-128.

299. Singh JP, Kandala J, Camm AJ. Non-pharmacological modulation of the autonomic tone to treat heart failure. Eur Heart J 2014;35:77-85.

300. Solomon SD, Zile M, Pieske B, Voors A, Shah A, Kraigher-Krainer E, Shi V, Bransford T, Takeuchi M, Gong J, Lefkowitz M, Packer M, McMurray JJ. The angiotensin receptor neprilysin inhibitor LCZ696 in heart failure with preserved ejection fraction: a phase 2 double-blind randomised controlled trial. Lancet 2012; 380:1387-1395.

301. Pitt B, Pfeffer MA, Assmann SF, Boineau R, Anand IS, Claggett B, Clausell N, Desai AS, Diaz R, Fleg JL, Gordeev I, Harty B, Heitner JF, Kenwood CT, Lewis EF, O'Meara E, Probstfield JL, Shaburishvili T, Shah SJ, Solomon SD, Sweitzer NK, Yang S, McKinlay SM. Spironolactone for heart failure with preserved ejection fraction. N EnglJ Med 2014;370:1383-1392.

302. Redfield MM, Chen HH, Borlaug BA, Semigran MJ, Lee KL, Lewis G, LeWinter MM, Rouleau JL, Bull DA, Mann DL, Deswal A, Stevenson LW, Givertz MM, Ofili EO, O'Connor CM, Felker GM, Goldsmith SR, Bart BA, McNulty SE, Ibarra JC, Lin G, Oh JK, Patel MR, Kim RJ, Tracy RP, Velazquez EJ, Anstrom KJ, Hernandez AF, Mascette AM, Braunwald E, RELAX Trial. Effect of phosphodiesterase-5 inhibition on exercise capacity and clinical status in heart failure with preserved ejection fraction: a randomized clinical trial. JAMA 2013; 309:1268-1277.

303. Senni M, Paulus WJ, Gavazzi A, Fraser AG, Diez J, Solomon SD, Smiseth OA, Guazzi M, Lam CSP, Maggioni AP, Tschope C, Metra M, Hummel SL, Edelmann F, Ambrosio G, Stewart Coats AJ, Filippatos GS, Gheorghiade M, Anker SD, Levy D, Pfeffer MA, Stough WG, Pieske BM. New strategies for heart failure with preserved ejection fraction: the importance of targeted therapies for heart failure phenotypes. Eur Heart J 2014;35:2797-2815.

304. Ferrari R, Böhm M, Cleland JGF, Paulus WJS, Pieske B, Rapezzi C, Tavazzi L. Heart failure with preserved ejection fraction: uncertainties and dilemmas. Eur J Heart Fail 2015;17:665-671.

305. Ather S, Chan W, Bozkurt B, Aguilar D, Ramasubbu K, Zachariah AA, Wehrens $\mathrm{XHT}$, Deswal A. Impact of noncardiac comorbidities on morbidity and mortality in a predominantly male population with heart failure and preserved versus reduced ejection fraction. J Am Coll Cardiol 2012;59:998-1005.

306. Henkel DM, Redfield MM, Weston SA, Gerber Y, Roger VL. Death in heart failure: a community perspective. Circ Heart Fail 2008;1:91-97.

307. Fukuta $\mathrm{H}$, Goto T, Wakami K, Ohte N. Effects of drug and exercise intervention on functional capacity and quality of life in heart failure with preserved ejection fraction: a meta-analysis of randomized controlled trials. Eur J Prev Cardiol 2016; 23:78-85.

308. Lewis EF, Lamas GA, O'Meara E, Granger CB, Dunlap ME, McKelvie RS, Probstfield JL, Young JB, Michelson EL, Halling K, Carlsson J, Olofsson B, McMurray JJV, Yusuf S, Swedberg K, Pfeffer MA. Characterization of health-related quality of life in heart failure patients with preserved versus low ejection fraction in CHARM. Eur J Heart Fail 2007;9:83-91.

309. Massie BM, Carson PE, McMurray JJ, Komajda M, McKelvie R, Zile MR, Anderson S, Donovan M, Iverson E, Staiger C, Ptaszynska A. Irbesartan in patients with heart failure and preserved ejection fraction. N Engl J Med 2008;359: 2456-2467.

310. Yusuf S, Pfeffer MA, Swedberg K, Granger CB, Held P, McMurray JJ V, Michelson EL, Olofsson B, Ostergren J. Effects of candesartan in patients with chronic heart failure and preserved left-ventricular ejection fraction: the CHARM-Preserved Trial. Lancet 2003;362:777-781.

311. Cleland JGF, Tendera M, Adamus J, Freemantle N, Polonski L, Taylor J. The perindopril in elderly people with chronic heart failure (PEP-CHF) study. Eur Heart $J$ 2006;27:2338-2345.

312. Mulder BA, van Veldhuisen DJ, Crijns HJGM, Böhm M, Cohen-Solal A, Babalis D, Roughton M, Flather MD, Coats AJS, Van Gelder IC. Effect of nebivolol on outcome in elderly patients with heart failure and atrial fibrillation: insights from SENIORS. Eur J Heart Fail 2012;14:1171-1178.

313. van Veldhuisen DJ, Cohen-Solal A, Böhm M, Anker SD, Babalis D, Roughton M, Coats AJS, Poole-Wilson PA, Flather MD. Beta-blockade with nebivolol in elderly heart failure patients with impaired and preserved left ventricular ejection fraction. J Am Coll Cardiol 2009;53:2150-2158.

314. Ahmed A, Rich MW, Fleg JL, Zile MR, Young JB, Kitzman DW, Love TE, Aronow WS, Adams KF, Gheorghiade M. Effects of digoxin on morbidity and mortality in diastolic heart failure: the ancillary digitalis investigation group trial. Circulation 2006;114:397-403.

315. McMurray JJ V, Ostergren J, Swedberg K, Granger CB, Held P, Michelson EL, Olofsson B, Yusuf S, Pfeffer MA. Effects of candesartan in patients with chronic heart failure and reduced left-ventricular systolic function taking angiotensin-converting-enzyme inhibitors: the CHARM-Added trial. Lancet 2003;362:767-771.

316. Kirchof P, Benussi S et al. 2016 ESC Guidelines for the management of atrial fibrillation developed in collaboration with EACTS. Eur Heart J. 2016. doi: 10.1093/ eurheartj/ehw210. Forthcoming.

317. Mancia G, Fagard R, Narkiewicz K, Redon J, Zanchetti A, Böhm M, Christiaens T, Cifkova R, De Backer G, Dominiczak A, Galderisi M, Grobbee DE, Jaarsma T, Kirchhof P, Kjeldsen SE, Laurent S, Manolis AJ, Nilsson PM, Ruilope LM, Schmieder RE, Sirnes PA, Sleight P, Viigimaa M, Waeber B, Zannad F, Redon J, Dominiczak A, Narkiewicz K, Nilsson PM, Burnier M, Viigimaa M, Ambrosioni E, Caufield M, Coca A, Olsen MH, Schmieder RE, Tsioufis C, van de Borne P, Zamorano JL, Achenbach S, Baumgartner H, Bax JJ, Bueno H, Dean V, Deaton C, Erol C, Fagard R, Ferrari R, Hasdai D, Hoes AW, Kirchhof P, Knuuti J, Kolh P, Lancellotti P, Linhart A, Nihoyannopoulos P, Piepoli MF, Ponikowski P, Sirnes PA, Tamargo JL, Tendera M, Torbicki A, Wijns W, Windecker S, Clement DL, Coca A, Gillebert TC, Tendera M, Rosei EA, Ambrosioni E, Anker SD, Bauersachs J, Hitij JB, Caulfield M, De Buyzere M, De Geest S, Derumeaux GA, Erdine S, Farsang C, Funck-Brentano C, Gerc V, Germano G, Gielen S, Haller H, Hoes AW, Jordan J, Kahan T, Komajda M, Lovic D, Mahrholdt H, Olsen MH, Ostergren J, Parati G, Perk J, Polonia J, Popescu BA, Reiner Z, Rydén L, Sirenko Y, Stanton A, Struijker-Boudier H, Tsioufis C, van de Borne P, Vlachopoulos C, Volpe M, Wood DA. 2013 ESH/ESC guidelines for the management of arterial hypertension: the Task Force for the Management of Arterial Hypertension of the European Society of Hypertension (ESH) and of the European Society of Cardiology (ESC). Eur Heart J 2013;34:2159-2219.

318. Sakata Y, Shiba N, Takahashi J, Miyata S, Nochioka K, Miura M, Takada T, Saga C, Shinozaki T, Sugi M, Nakagawa M, Sekiguchi N, Komaru T, Kato A, Fukuchi M, Nozaki E, Hiramoto T, Inoue K, Goto T, Ohe M, Tamaki K, Ibayashi S, Ishide N, Maruyama Y, Tsuji I, Shimokawa H. Clinical impacts of additive use of olmesartan in hypertensive patients with chronic heart failure: the supplemental benefit of an angiotensin receptor blocker in hypertensive patients with stable heart failure using olmesartan (SUPPORT) trial. Eur Heart / 2015;36:915-923.

319. Rydén L, Grant PJ, Anker SD, Berne C, Cosentino F, Danchin N, Deaton C, Escaned J, Hammes H-P, Huikuri H, Marre M, Marx N, Mellbin L, Ostergren J, Patrono C, Seferovic P, Uva MS, Taskinen M-R, Tendera M, Tuomilehto J, Valensi P, Zamorano JL, Achenbach S, Baumgartner H, Bax JJ, Bueno $\mathrm{H}$, Dean V, Erol C, Fagard R, Ferrari R, Hasdai D, Hoes AW, Kirchhof P, Knuuti J, Kolh P, Lancellotti P, Linhart A, Nihoyannopoulos P, Piepoli MF, Ponikowski P, Sirnes PA, Tamargo JL, Torbicki A, Wijns W, Windecker S, De Backer G, Ezquerra EA, Avogaro A, Badimon L, Baranova E, Betteridge J, Ceriello A, Funck-Brentano C, Gulba DC, Kjekshus JK, Lev E, Mueller C, Neyses L, Nilsson PM, Perk J, Reiner Z, Sattar N, Schächinger V, Scheen A, Schirmer H, Strömberg A, Sudzhaeva S, Viigimaa M, Vlachopoulos C, Xuereb RG. ESC Guidelines on diabetes, pre-diabetes, and cardiovascular diseases developed in collaboration with the EASD. Eur Heart J 2013;34:3035-3087.

320. Gilbert RE, Krum H. Heart failure in diabetes: effects of anti-hyperglycaemic drug therapy. Lancet 2015;385:2107-2117.

321. Edelmann F, Gelbrich G, Düngen H-D, Fröhling S, Wachter R, Stahrenberg R, Binder L, Töpper A, Lashki DJ, Schwarz S, Herrmann-Lingen C, Löffler M, Hasenfuss G, Halle M, Pieske B. Exercise training improves exercise capacity 
and diastolic function in patients with heart failure with preserved ejection fraction. J Am Coll Cardiol 2011;58:1780-1791.

322. Katritsis DG, Siontis GCM, Camm AJ. Prognostic significance of ambulatory ECG monitoring for ventricular arrhythmias. Prog Cardiovasc Dis 2013;56:133-142.

323. Camm AJ, Corbucci G, Padeletti L. Usefulness of continuous electrocardiographic monitoring for atrial fibrillation. Am J Cardiol 2012;110:270-276.

324. Turakhia MP, Ullal AJ, Hoang DD, Than CT, Miller JD, Friday KJ, Perez M V, Freeman JV, Wang PJ, Heidenreich PA. Feasibility of extended ambulatory electrocardiogram monitoring to identify silent atrial fibrillation in high-risk patients: the Screening Study for Undiagnosed Atrial Fibrillation (STUDY-AF). Clin Cardiol 2015;38:285-292.

325. Camm AJ, Kirchhof P, Lip GYH, Schotten U, Savelieva I, Ernst S, Van Gelder IC, Al-Attar N, Hindricks G, Prendergast B, Heidbuchel H, Alfieri O, Angelini A, Atar D, Colonna P, De Caterina R, De Sutter J, Goette A, Gorenek B, Heldal M, Hohloser SH, Kolh P, Le Heuzey JY, Ponikowski P, Rutten FH, Vahanian A, Auricchio A, Bax J, Ceconi C, Dean V, Filippatos G, Funck-Brentano C, Hobbs R, Kearney P, McDonagh T, Popescu BA, Reiner Z, Sechtem U, Sirnes PA, Tendera M, Vardas PE, Widimsky P, Agladze V, Aliot E, Balabanski T, Blomstrom-Lundqvist C, Capucci A, Crijns H, Dahlof B, Folliguet T, Glikson M, Goethals M, Gulba DC, Ho SY, Klautz RJM, Kose S, McMurray J, PerroneFilardi P, Raatikainen P, Salvador MJ, Schalij MJ, Shpektor A, Sousa J, Stepinska J, Uuetoa H, Zamorano JL, Zupan I. Guidelines for the management of atrial fibrillation: the Task Force for the Management of Atrial Fibrillation of the European Society of Cardiology (ESC). Eur Heart J 2010;31:2369-2429.

326. Cintra FD, Leite RP, Storti LJ, Bittencourt LA, Poyares D, Castro L de S, Tufik S, Paola A de. Sleep apnea and nocturnal cardiac arrhythmia: a populational study. Arq Bras Cardiol 2014;103:368-374.

327. Gilat H, Vinker S, Buda I, Soudry E, Shani M, Bachar G. Obstructive sleep apnea and cardiovascular comorbidities: a large epidemiologic study. Medicine (Baltimore) 2014;93:e45.

328. Vizzardi E, Sciatti E, Bonadei I, D’Aloia A, Curnis A, Metra M. Obstructive sleep apnoea-hypopnoea and arrhythmias. J Cardiovasc Med 2014;1.

329. Bloch Thomsen PE, Jons C, Raatikainen MJP, MoerchJoergensen R, Hartikainen J, Virtanen V, Boland J, Anttonen O, Gang UJ, Hoest N, Boersma LVA, Platou ES, Becker D, Messier MD, Huikuri HV. Long-term recording of cardiac arrhythmias with an implantable cardiac monitor in patients with reduced ejection fraction after acute myocardial infarction: the Cardiac Arrhythmias and Risk Stratification After Acute Myocardial Infarction (CARISMA) study. Circulation 2010;122: 1258-1264.

330. Faggiano P, D'Aloia A, Gualeni A, Gardini A, Giordano A. Mechanisms and immediate outcome of in-hospital cardiac arrest in patients with advanced heart failure secondary to ischemic or idiopathic dilated cardiomyopathy. Am J Cardiol 2001;87: 655-657, A10-A11.

331. Smit MD, Moes ML, Maass AH, Achekar ID, Van Geel PP, Hillege HL, van Veldhuisen DJ, Van Gelder IC. The importance of whether atrial fibrillation or heart failure develops first. Eur J Heart Fail 2012;14:1030-1040.

332. Swedberg K, Olsson LG, Charlesworth A, Cleland J, Hanrath P, Komajda M, Metra M, Torp-Pedersen C, Poole-Wilson P. Prognostic relevance of atrial fibrillation in patients with chronic heart failure on long-term treatment with betablockers: results from COMET. Eur Heart J 2005;26:1303-1308.

333. Hoppe UC, Casares JM, Eiskjaer H, Hagemann A, Cleland JGF, Freemantle N, Erdmann $\mathrm{E}$. Effect of cardiac resynchronization on the incidence of atrial fibrillation in patients with severe heart failure. Circulation 2006;114:18-25.

334. Calvo N, Bisbal F, Guiu E, Ramos P, Nadal M, Tolosana JM, Arbelo E, Berruezo A, Sitges M, Brugada J, Mont L. Impact of atrial fibrillation-induced tachycardiomyopathy in patients undergoing pulmonary vein isolation. Int J Cardiol 2013;168: 4093-4097.

335. Morris PD, Robinson T, Channer KS. Reversible heart failure: toxins, tachycardiomyopathy and mitochondrial abnormalities. Postgrad Med J 2012;88:706-712.

336. Pedersen OD, Bagger H, Køber L, Torp-Pedersen C. Trandolapril reduces the incidence of atrial fibrillation after acute myocardial infarction in patients with left ventricular dysfunction. Circulation 1999;100:376-380.

337. Ducharme A, Swedberg K, Pfeffer MA, Cohen-Solal A, Granger CB, Maggioni AP, Michelson EL, McMurray JJ V, Olsson L, Rouleau JL, Young JB, Olofsson B, Puu M, Yusuf S. Prevention of atrial fibrillation in patients with symptomatic chronic heart failure by candesartan in the Candesartan in Heart failure: Assessment of Reduction in Mortality and morbidity (CHARM) program. Am Heart J 2006;152:86-92.

338. McMurray J, Køber L, Robertson M, Dargie H, Colucci W, Lopez-Sendon J, Remme W, Sharpe DN, Ford I. Antiarrhythmic effect of carvedilol after acute myocardial infarction. J Am Coll Cardiol 2005;45:525-530.

339. Swedberg K, Zannad F, McMurray JJV, Krum H, van Veldhuisen DJ, Shi H, Vincent J, Pitt B. Eplerenone and atrial fibrillation in mild systolic heart failure. J Am Coll Cardiol 2012;59:1598-1603.
340. Han M, Zhang Y, Sun S, Wang Z, Wang J, Xie X, Gao M, Yin X, Hou Y. Reninangiotensin system inhibitors prevent the recurrence of atrial fibrillation. J Cardiovasc Pharmacol 2013;62:405-415.

341. Martin RIR, Pogoryelova O, Koref MS, Bourke JP, Teare MD, Keavney BD. Atrial fibrillation associated with ivabradine treatment: meta-analysis of randomised controlled trials. Heart 2014;100:1506-1510.

342. Hess PL, Jackson KP, Hasselblad V, Al-Khatib SM. Is cardiac resynchronization therapy an antiarrhythmic therapy for atrial fibrillation? A systematic review and meta-analysis. Curr Cardiol Rep 2013;15:330.

343. Brodsky MA, Allen BJ, Walker CJ, Casey TP, Luckett CR, Henry WL. Amiodarone for maintenance of sinus rhythm after conversion of atrial fibrillation in the setting of a dilated left atrium. Am J Cardiol 1987;60:572-575.

344. Deedwania PC, Singh BN, Ellenbogen K, Fisher S, Fletcher R, Singh SN. Spontaneous conversion and maintenance of sinus rhythm by amiodarone in patients with heart failure and atrial fibrillation: observations from the veterans affairs congestive heart failure survival trial of antiarrhythmic therapy (CHF-STAT). The Department of Veterans Affairs CHF-STAT Investigators. Circulation 1998;98: 2574-2579.

345. Shelton RJ, Clark AL, Goode K, Rigby AS, Houghton T, Kaye GC, Cleland JGF. A randomised, controlled study of rate versus rhythm control in patients with chronic atrial fibrillation and heart failure: (CAFE-II Study). Heart 2009;95: 924-930.

346. Capucci A, Villani GQ, Aschieri D, Rosi A, Piepoli MF. Oral amiodarone increases the efficacy of direct-current cardioversion in restoration of sinus rhythm in patients with chronic atrial fibrillation. Eur Heart J 2000;21:66-73.

347. Connolly SJ, Camm AJ, Halperin JL, Joyner C, Alings M, Amerena J, Atar D, Avezum Á, Blomström P, Borggrefe M, Budaj A, Chen S-A, Ching CK, Commerford P, Dans A, Davy J-M, Delacrétaz E, Di Pasquale G, Diaz R, Dorian P, Flaker G, Golitsyn S, Gonzalez-Hermosillo A, Granger CB, Heidbüchel H, Kautzner J, Kim JS, Lanas F, Lewis BS, Merino JL, Morillo C, Murin J, Narasimhan C, Paolasso E, Parkhomenko A, Peters NS, Sim K-H, Stiles MK, Tanomsup S, Toivonen L, Tomcsányi J, Torp-Pedersen C, Tse H-F, Vardas P, Vinereanu D, Xavier D, Zhu J-R, Zhu J-R, Baret-Cormel L, Weinling E, Staiger C, Yusuf S, Chrolavicius S, Afzal R, Hohnloser SH. Dronedarone in high-risk permanent atrial fibrillation. N Engl J Med 2011;365:2268-2276.

348. Hofmann R, Steinwender C, Kammler J, Kypta A, Leisch F. Effects of a high dose intravenous bolus amiodarone in patients with atrial fibrillation and a rapid ventricular rate. Int J Cardiol 2006;110:27-32.

349. Hofmann R, Wimmer G, Leisch F. Intravenous amiodarone bolus immediately controls heart rate in patients with atrial fibrillation accompanied by severe congestive heart failure. Heart 2000;84:635.

350. Li S-J, Sartipy U, Lund LH, Dahlström U, Adiels M, Petzold M, Fu M. Prognostic significance of resting heart rate and use of $\beta$-blockers in atrial fibrillation and sinus rhythm in patients with heart failure and reduced ejection fraction: findings from the Swedish Heart Failure Registry. Circ Heart Fail 2015;8:871-879.

351. Mareev Y, Cleland JGF. Should $\beta$-blockers be used in patients with heart failure and atrial fibrillation? Clin Ther 2015;37:2215-2224.

352. Hagens VE, Crijns HJGM, Van Veldhuisen DJ, Van Den Berg MP, Rienstra M, Ranchor AV, Bosker HA, Kamp O, Tijssen JGP, Veeger NJGM, Van Gelder IC. Rate control versus rhythm control for patients with persistent atrial fibrillation with mild to moderate heart failure: results from the RAte Control versus Electrical cardioversion (RACE) study. Am Heart J 2005;149:1106-1111.

353. Van Gelder IC, Hagens VE, Bosker HA, Kingma JH, Kamp O, Kingma T, Said SA, Darmanata JI, Timmermans AJM, Tijssen JGP, Crijns HJGM. A comparison of rate control and rhythm control in patients with recurrent persistent atrial fibrillation. N Engl J Med 2002;347:1834-1840.

354. Van Gelder IC, Wyse DG, Chandler ML, Cooper HA, Olshansky B, Hagens VE, Crijns HJGM, RACE and AFFIRM Investigators. Does intensity of rate-control influence outcome in atrial fibrillation? An analysis of pooled data from the RACE and AFFIRM studies. Europace 2006;8:935-942.

355. Allen LA, Fonarow GC, Simon DN, Thomas LE, Marzec LN, Pokorney SD, Gersh BJ, Go AS, Hylek EM, Kowey PR, Mahaffey KW, Chang P, Peterson ED, Piccini JP. Digoxin use and subsequent outcomes among patients in a contemporary atrial fibrillation cohort. J Am Coll Cardiol 2015;65:2691-2698.

356. Gheorghiade M, Fonarow GC, van Veldhuisen DJ, Cleland JGF, Butler J, Epstein AE, Patel K, Aban IB, Aronow WS, Anker SD, Ahmed A. Lack of evidence of increased mortality among patients with atrial fibrillation taking digoxin: findings from post hoc propensity-matched analysis of the AFFIRM trial. Eur Heart J 2013;34:1489-1497.

357. Turakhia MP, Santangeli P, Winkelmayer WC, Xu X, Ullal AJ, Than CT, Schmitt S, Holmes TH, Frayne SM, Phibbs CS, Yang F, Hoang DD, Ho PM, Heidenreich PA. Increased mortality associated with digoxin in contemporary patients with atrial fibrillation. J Am Coll Cardiol 2014;64:660-668. 
358. Khand AU, Rankin AC, Martin W, Taylor J, Gemmell I, Cleland JGF. Carvedilol alone or in combination with digoxin for the management of atrial fibrillation in patients with heart failure? J Am Coll Cardiol 2003;42:1944-1951.

359. Roy D, Talajic M, Nattel S, Wyse DG, Dorian P, Lee KL, Bourassa MG, Arnold JMO, Buxton AE, Camm AJ, Connolly SJ, Dubuc M, Ducharme A, Guerra PG, Hohnloser SH, Lambert J, Le Heuzey J-Y, O'Hara G, Pedersen OD, Rouleau J-L, Singh BN, Stevenson LW, Stevenson WG, Thibault B, Waldo AL. Rhythm control versus rate control for atrial fibrillation and heart failure. $N$ Engl J Med 2008;358:2667-2677.

360. Khan MN, Jais P, Cummings J, Di Biase L, Sanders P, Martin DO, Kautzner J, Hao S, Themistoclakis S, Fanelli R, Potenza D, Massaro R, Wazni O, Schweikert R, Saliba W, Wang P, Al-Ahmad A, Beheiry S, Santarelli P, Starling RC, Dello Russo A, Pelargonio G, Brachmann J, Schibgilla V, Bonso A, Casella M, Raviele A, Haissaguerre M, Natale A. Pulmonary-vein isolation for atrial fibrillation in patients with heart failure. N Engl J Med 2008;359:1778-1785.

361. Ganesan AN, Nandal S, Lüker J, Pathak RK, Mahajan R, Twomey D, Lau DH, Sanders P. Catheter ablation of atrial fibrillation in patients with concomitant left ventricular impairment: a systematic review of efficacy and effect on ejection fraction. Heart Lung Circ 2015;24:270-280.

362. Marrouche NF, Brachmann J. Catheter ablation versus standard conventional treatment in patients with left ventricular dysfunction and atrial fibrillation (CASTLE-AF) - study design. Pacing Clin Electrophysiol 2009;32:987-994.

363. Khan AR, Khan S, Sheikh MA, Khuder S, Grubb B, Moukarbel GV. Catheter ablation and antiarrhythmic drug therapy as first- or second-line therapy in the management of atrial fibrillation: systematic review and meta-analysis. Circ Arrhythm Electrophysiol 2014;7:853-860.

364. Lafuente-Lafuente C, Valembois L, Bergmann J-F, Belmin J. Antiarrhythmics for maintaining sinus rhythm after cardioversion of atrial fibrillation. Cochrane Database Syst Rev 2012;5:CD005049.

365. A multicentre, randomized trial on the benefit/risk profile of amiodarone, flecainide and propafenone in patients with cardiac disease and complex ventricular arrhythmias. Antiarrhythmic Drug Evaluation Group (A.D.E.G.). Eur Heart J 1992; 13:1251-1258.

366. Ruff CT, Giugliano RP, Braunwald E, Hoffman EB, Deenadayalu N, Ezekowitz MD, Camm AJ, Weitz JI, Lewis BS, Parkhomenko A, Yamashita T, Antman EM. Comparison of the efficacy and safety of new oral anticoagulants with warfarin in patients with atrial fibrillation: a meta-analysis of randomised trials. Lancet 2014;383: 955-962.

367. Xiong Q, Lau YC, Senoo K, Lane DA, Hong K, Lip GYH. Non-vitamin K antagonist oral anticoagulants (NOACs) in patients with concomitant atrial fibrillation and heart failure: a systemic review and meta-analysis of randomized trials. Eur J Heart Fail 2015;17:1192-1200.

368. Sardar P, Chatterjee S, Lavie CJ, Giri JS, Ghosh J, Mukherjee D, Lip GYH. Risk of major bleeding in different indications for new oral anticoagulants: insights from a meta-analysis of approved dosages from 50 randomized trials. Int J Cardiol 2015; 179:279-287.

369. Sardar P, Chatterjee S, Chaudhari S, Lip GYH. New oral anticoagulants in elderly adults: evidence from a meta-analysis of randomized trials. J Am Geriatr Soc 2014; 62:857-864.

370. Heidbuchel H, Verhamme P, Alings M, Antz M, Diener H-C, Hacke W, Oldgren J, Sinnaeve P, Camm AJ, Kirchhof P. Updated European Heart Rhythm Association practical guide on the use of non-vitamin $\mathrm{K}$ antagonist anticoagulants in patients with non-valvular atrial fibrillation. Europace 2015;17:1467-1507.

371. Connolly SJ, Ezekowitz MD, Yusuf S, Eikelboom J, Oldgren J, Parekh A, Pogue J, Reilly PA, Themeles E, Varrone J, Wang S, Alings M, Xavier D, Zhu J, Diaz R, Lewis BS, Darius H, Diener H-C, Joyner CD, Wallentin L. Dabigatran versus warfarin in patients with atrial fibrillation. N Engl J Med 2009;361:1139-1151.

372. Patel MR, Mahaffey KW, Garg J, Pan G, Singer DE, Hacke W, Breithardt G, Halperin JL, Hankey GJ, Piccini JP, Becker RC, Nessel CC, Paolini JF, Berkowitz SD, Fox KAA, Califf RM. Rivaroxaban versus warfarin in nonvalvular atrial fibrillation. N Engl J Med 2011;365:883-891.

373. Giugliano RP, Ruff CT, Braunwald E, Murphy SA, Wiviott SD, Halperin JL, Waldo AL, Ezekowitz MD, Weitz JI, Špinar J, Ruzyllo W, Ruda M, Koretsune Y, Betcher J, Shi M, Grip LT, Patel SP, Patel I, Hanyok JJ, Mercuri M, Antman EM. Edoxaban versus warfarin in patients with atrial fibrillation. N Engl J Med 2013; 369:2093-2104.

374. Granger CB, Alexander JH, McMurray J V , Lopes RD, Hylek EM, Hanna M, Al-Khalidi HR, Ansell J, Atar D, Avezum A, Bahit MC, Diaz R, Easton JD, Ezekowitz JA, Flaker G, Garcia D, Geraldes M, Gersh BJ, Golitsyn S, Goto S, Hermosillo AG, Hohnloser SH, Horowitz J, Mohan P, Jansky P, Lewis BS, Lopez-Sendon JL, Pais P, Parkhomenko A, Verheugt FWA, Zhu J, Wallentin L. Apixaban versus warfarin in patients with atrial fibrillation. N Engl J Med 2011; 365:981-992.

375. Connolly SJ, Eikelboom J, Joyner C, Diener H-C, Hart R, Golitsyn S, Flaker G, Avezum A, Hohnloser SH, Diaz R, Talajic M, Zhu J, Pais P, Budaj A,
Parkhomenko A, Jansky P, Commerford P, Tan RS, Sim K-H, Lewis BS, Van Mieghem W, Lip GYH, Kim JH, Lanas-Zanetti F, Gonzalez-Hermosillo A, Dans AL, Munawar M, O’Donnell M, Lawrence J, Lewis G, Afzal R, Yusuf S. Apixaban in patients with atrial fibrillation. N Engl J Med 2011;364:806-817.

376. Lip GYH, Nieuwlaat R, Pisters R, Lane DA, Crijns HJGM. Refining clinical risk stratification for predicting stroke and thromboembolism in atrial fibrillation using a novel risk factor-based approach: the Euro Heart Survey on atrial fibrillation. Chest 2010;137:263-272.

377. Pisters R, Lane DA, Nieuwlaat R. A novel user-friendly score (HAS BLED) to asses 1 -year risk of major bleeding in patients with atrial fibrillation. Chest J 2010;138: 1093-1100.

378. Connolly SJ, Ezekowitz MD, Yusuf S, Reilly PA, Wallentin L. Newly identified events in the RE-LY trial. N Engl J Med 2010;363:1875-1876.

379. Hart RG, Pearce LA, Aguilar MI. Meta-analysis: antithrombotic therapy to prevent stroke in patients who have nonvalvular atrial fibrillation. Ann Intern Med 2007; 146:857-867.

380. Eikelboom JW, Connolly SJ, Brueckmann M, Granger CB, Kappetein AP, Mack MJ, Blatchford J, Devenny K, Friedman J, Guiver K, Harper R, Khder Y, Lobmeyer MT, Maas H, Voigt J-U, Simoons ML, Van de Werf F. Dabigatran versus warfarin in patients with mechanical heart valves. N Engl J Med 2013;369:1206-1214.

381. Holmes DR, Doshi SK, Kar S, Price MJ, Sanchez JM, Sievert H, Valderrabano M, Reddy VY. Left atrial appendage closure as an alternative to warfarin for stroke prevention in atrial fibrillation: a patient-level meta-analysis. J Am Coll Cardiol 2015;65:2614-2623.

382. Price MJ, Reddy VY, Valderrábano M, Halperin JL, Gibson DN, Gordon N, Huber KC, Holmes DR. Bleeding outcomes after left atrial appendage closure compared with long-term warfarin: a pooled, patient-level analysis of the WATCHMAN randomized trial experience. JACC Cardiovasc Interv 2015;8: 1925-1932.

383. Cleland JG, Massie BM, Packer M. Sudden death in heart failure: vascular or electrical? Eur J Heart Fail 1999;1:41-45.

384. Desai AS, McMurray JJ V, Packer M, Swedberg K, Rouleau JL, Chen F, Gong J, Rizkala AR, Brahimi A, Claggett B, Finn P V, Hartley LH, Liu J, Lefkowitz M, Shi V, Zile MR, Solomon SD. Effect of the angiotensin-receptor-neprilysin inhibitor LCZ696 compared with enalapril on mode of death in heart failure patients. Eur Heart J 2015;36:1990-1997.

385. Velazquez EJ, Lee KL, Deja MA, Jain A, Sopko G, Marchenko A, Ali IS, Pohost G, Gradinac S, Abraham WT, Yii M, Prabhakaran D, Szwed H, Ferrazzi P, Petrie MC, O'Connor CM, Panchavinnin P, She L, Bonow RO, Rankin GR, Jones RH, Rouleau J-L, STICH Investigators. Coronary-artery bypass surgery in patients with left ventricular dysfunction. N Engl J Med 2011;364:1607-1616.

386. Panza JA, Holly TA, Asch FM, She L, Pellikka PA, Velazquez EJ, Lee KL, Borges-Neto S, Farsky PS, Jones RH, Berman DS, Bonow RO. Inducible myocardial ischemia and outcomes in patients with coronary artery disease and left ventricular dysfunction. J Am Coll Cardiol 2013;61:1860-1870.

387. Carson P, Wertheimer J, Miller A, O'Connor CM, Pina IL, Selzman C, Sueta C, She L, Greene D, Lee KL, Jones RH, Velazquez EJ. The STICH trial (Surgical Treatment for Ischemic Heart Failure): mode-of-death results. JACC Heart Fail 2013;1: 400-408.

388. Oseroff O, Retyk E, Bochoeyer A. Subanalyses of secondary prevention implantable cardioverter-defibrillator trials: antiarrhythmics versus implantable defibrillators (AVID), Canadian Implantable Defibrillator Study (CIDS), and Cardiac Arrest Study Hamburg (CASH). Curr Opin Cardiol 2004;19:26-30.

389. Brignole M, Auricchio A, Baron-Esquivias G, Bordachar P, Boriani G, Breithardt O-A, Cleland J, Deharo J-C, Delgado V, Elliott PM, Gorenek B, Israel CW, Leclercq C, Linde C, Mont L, Padeletti L, Sutton R, Vardas PE, Zamorano JL, Achenbach S, Baumgartner H, Bax JJ, Bueno H, Dean V, Deaton C, Erol C, Fagard R, Ferrari R, Hasdai D, Hoes AW, Kirchhof P, Knuuti J, Kolh P, Lancellotti P, Linhart A, Nihoyannopoulos P, Piepoli MF, Ponikowski P, Sirnes PA, Tamargo JL, Tendera M, Torbicki A, Wijns W, Windecker S, Blomstrom-Lundqvist C, Badano LP, Aliyev F, Bänsch D, Bsata W, Buser P, Charron P, Daubert J-C, Dobreanu D, Faerestrand S, Le Heuzey J-Y, Mavrakis H, McDonagh T, Merino JL, Nawar MM, Nielsen JC, Pieske B, Poposka L, Ruschitzka F, Van Gelder IC, Wilson CM. 2013 ESC Guidelines on cardiac pacing and cardiac resynchronization therapy: the Task Force on cardiac pacing and resynchronization therapy of the European Society of Cardiology (ESC). Developed in collaboration with the European Heart Rhythm Association. Eur Heart J 2013;34:2281-2329.

390. Blondé-Cynober F, Morineau G, Estrugo B, Fillie E, Aussel C, Vincent J-P. Diagnostic and prognostic value of brain natriuretic peptide (BNP) concentrations in very elderly heart disease patients: specific geriatric cut-off and impacts of age, gender, renal dysfunction, and nutritional status. Arch Gerontol Geriatr 2011;52:106-110.

391. Hawkins NM, Virani S, Ceconi C. Heart failure and chronic obstructive pulmonary disease: the challenges facing physicians and health services. Eur Heart J 2013;34: 2795-2807. 
392. Enjuanes C, Klip IT, Bruguera J, Cladellas M, Ponikowski P, Banasiak W, van Veldhuisen DJ, van der Meer P, Jankowska EA, Comín-Colet J. Iron deficiency and health-related quality of life in chronic heart failure: results from a multicenter European study. Int J Cardiol 2014;174:268-275.

393. Braunstein JB, Anderson GF, Gerstenblith G, Weller W, Niefeld M, Herbert R, Wu AW. Noncardiac comorbidity increases preventable hospitalizations and mortality among Medicare beneficiaries with chronic heart failure. J Am Coll Cardiol 2003;42:1226-1233.

394. Muzzarelli S, Leibundgut G, Maeder MT, Rickli H, Handschin R, Gutmann M, Jeker U, Buser P, Pfisterer M, Brunner-La Rocca H-P. Predictors of early readmission or death in elderly patients with heart failure. Am Heart J 2010;160:308-314.

395. Reddel HK, Bateman ED, Becker A, Boulet L-P, Cruz AA, Drazen JM, Haahtela T, Hurd SS, Inoue H, de Jongste JC, Lemanske RF, Levy ML, O'Byrne PM, Paggiaro P, Pedersen SE, Pizzichini E, Soto-Quiroz M, Szefler SJ, Wong GWK, FitzGerald JM. A summary of the new GINA strategy: a roadmap to asthma control. Eur Respir 」 2015;46:622-639.

396. Global Initiative for Asthma. Global Strategy for Asthma Management and Prevention, Available at http://ginasthma.org/gina-report-global-strategy-for-asthmamanagement-and-prevention (date last accessed 12 February 2016).

397. Eschenhagen T, Force T, Ewer MS, De Keulenaer GW, Suter TM, Anker SD, Avkiran M, De Azambuja E, Balligand JL, Brutsaert DL, Condorelli G, Hansen A, Heymans S, Hill J a, Hirsch E, Hilfiker-Kleiner D, Janssens S, De Jong S, Neubauer G, Pieske B, Ponikowski P, Pirmohamed M, Rauchhaus M, Sawyer D, Sugden PH, Wojta J, Zannad F, Shah AM. Cardiovascular side effects of cancer therapies: a position statement from the Heart Failure Association of the European Society of Cardiology. Eur J Heart Fail 2011;13:1-10.

398. Paulus WJ, Tschöpe C. A novel paradigm for heart failure with preserved ejection fraction. J Am Coll Cardiol 2013;62:263-271.

399. Fox K, Ford I, Steg PG, Tardif J-C, Tendera M, Ferrari R. Ivabradine in stable coronary artery disease without clinical heart failure. N Engl J Med 2014;371: 1091-1099.

400. Marazzi G, Wajngarten M, Vitale C, Patrizi R, Pelliccia F, Gebara O, Pierri H, Ramires JAF, Volterrani M, Fini M, Rosano GMC. Effect of free fatty acid inhibition on silent and symptomatic myocardial ischemia in diabetic patients with coronary artery disease. Int J Cardiol 2007;120:79-84.

401. Vitale C, Spoletini I, Malorni W, Perrone-Filardi P, Volterrani M, Rosano GMC. Efficacy of trimetazidine on functional capacity in symptomatic patients with stable exertional angina - the VASCO-angina study. Int J Cardiol 2013;168:1078-1081.

402. Vitale C, Wajngaten M, Sposato B, Gebara O, Rossini P, Fini M, Volterrani M, Rosano GMC. Trimetazidine improves left ventricular function and quality of life in elderly patients with coronary artery disease. Eur Heart J 2004;25: 1814-1821.

403. Gao D, Ning N, Niu X, Hao G, Meng Z. Trimetazidine: a meta-analysis of randomised controlled trials in heart failure. Heart 2011;97:278-286.

404. Tuunanen $H$, Engblom E, Naum A, Någren K, Scheinin M, Hesse B, Juhani Airaksinen KE, Nuutila P, lozzo P, Ukkonen H, Opie LH, Knuuti J. Trimetazidine, a metabolic modulator, has cardiac and extracardiac benefits in idiopathic dilated cardiomyopathy. Circulation 2008;118:1250-1258.

405. Marazzi G, Gebara O, Vitale C, Caminiti G, Wajngarten M, Volterrani M, Ramires JAF, Rosano G, Fini M. Effect of trimetazidine on quality of life in elderly patients with ischemic dilated cardiomyopathy. Adv Ther 2009;26:455-461.

406. Rosano G, Vitale C, Sposato B, Mercuro G, Fini M. Trimetazidine improves left ventricular function in diabetic patients with coronary artery disease: a doubleblind placebo-controlled study. Cardiovasc Diabetol 2003;2:16.

407. Wijeysundera HC, Hansen MS, Stanton E, Cropp a. S, Hall C, Dhalla NS, Ghali J, Rouleau JL. Neurohormones and oxidative stress in nonischemic cardiomyopathy: Relationship to survival and the effect of treatment with amlodipine. Am Heart J 2003;146:291-297.

408. IONA Study Group. Effect of nicorandil on coronary events in patients with stable angina: the Impact Of Nicorandil in Angina (IONA) randomised trial. Lancet 2002; 359:1269-1275.

409. Cohn JN, Johnson G, Ziesche S, Cobb F, Francis G, Tristani F, Smith R, Dunkman WB, Loeb H, Wong M, Bhat G, Goldman S, Fletcher RD, Doherty J, Hughes CV, Carson P, Cintron G, Shabetai R, Haakenson C. A comparison of enalapril with hydralazine-isosorbide dinitrate in the treatment of chronic congestive heart failure. N Engl J Med 1991;325:303-310.

410. Fox K, Ford I, Steg PG, Tendera M, Ferrari R. Ivabradine for patients with stable coronary artery disease and left-ventricular systolic dysfunction (BEAUTIFUL): a randomised, double-blind, placebo-controlled trial. Lancet 2008;372:807-816.

411. Fox K, Ford I, Steg PG, Tendera M, Robertson M, Ferrari R. Relationship between ivabradine treatment and cardiovascular outcomes in patients with stable coronary artery disease and left ventricular systolic dysfunction with limiting angina: a subgroup analysis of the randomized, controlled BEAUTIFUL trial. Eur Heart J 2009;30:2337-2345.
412. Pursnani S, Korley F, Gopaul R, Kanade P, Chandra N, Shaw RE, Bangalore S. Percutaneous coronary intervention versus optimal medical therapy in stable coronary artery disease: a systematic review and meta-analysis of randomized clinical trials. Circ Cardiovasc Interv 2012;5:476-490.

413. Trikalinos TA, Alsheikh-Ali AA, Tatsioni A, Nallamothu BK, Kent DM. Percutaneous coronary interventions for non-acute coronary artery disease: a quantitative 20-year synopsis and a network meta-analysis. Lancet 2009;373:911-918.

414. von Haehling S, Anker SD. Prevalence, incidence and clinical impact of cachexia: facts and numbers-update 2014. J Cachexia Sarcopenia Muscle 2014;5:261-263.

415. von Haehling S, Lainscak M, Springer J, Anker SD. Cardiac cachexia: a systematic overview. Pharmacol Ther 2009;121:227-252.

416. Evans WJ, Morley JE, Argilés J, Bales C, Baracos V, Guttridge D, Jatoi A, Kalantar-Zadeh K, Lochs H, Mantovani G, Marks D, Mitch WE, Muscaritoli M, Najand A, Ponikowski P, Rossi Fanelli F, Schambelan M, Schols A, Schuster M, Thomas D, Wolfe R, Anker SD. Cachexia: a new definition. Clin Nutr 2008;27: 793-799.

417. Akashi YJ, Springer J, Anker SD. Cachexia in chronic heart failure: prognostic implications and novel therapeutic approaches. Curr Heart Fail Rep 2005;2:198-203.

418. Anker SD, Chua TP, Ponikowski P, Harrington D, Swan JW, Kox WJ, Poole-Wilson PA, Coats AJ. Hormonal changes and catabolic/anabolic imbalance in chronic heart failure and their importance for cardiac cachexia. Circulation 1997; 96:526-534.

419. Fülster S, Tacke M, Sandek A, Ebner N, Tschöpe C, Doehner W, Anker SD, Von Haehling S. Muscle wasting in patients with chronic heart failure: results from the studies investigating co-morbidities aggravating heart failure (SICA-HF). Eur Heart J 2013;34:512-519.

420. Morley JE, Anker SD, von Haehling S. Prevalence, incidence, and clinical impact of sarcopenia: facts, numbers, and epidemiology-update 2014. J Cachexia Sarcopenia Muscle 2014;5:253-259.

421. von Haehling S, Anker SD. Treatment of cachexia: an overview of recent developments. J Am Med Dir Assoc 2014;15:866-872.

422. Jones AL, Barlow M, Barrett-Lee PJ, Canney PA, Gilmour IM, Robb SD, Plummer CJ, Wardley AM, Verrill MW. Management of cardiac health in trastuzumab-treated patients with breast cancer: updated United Kingdom National Cancer Research Institute recommendations for monitoring. $\mathrm{Br} J$ Cancer 2009;100:684-692.

423. van Dalen EC, Caron HN, Dickinson HO, Kremer LCM. Cardioprotective interventions for cancer patients receiving anthracyclines. Cochrane Database Syst Rev 2005;6:CD003917.

424. Ezaz G, Long JB, Gross CP, Chen J. Risk prediction model for heart failure and cardiomyopathy after adjuvant trastuzumab therapy for breast cancer. J Am Heart Assoc 2014;3:e000472.

425. Suter TM, Ewer MS. Cancer drugs and the heart: Importance and management. Eur Heart J 2013;34:1102-1111.

426. Zamorano J-L, Lancelotti $P$ et al. 2016 Position paper on anticancer treatments and cardiovascular toxicity. Eur Heart J. 2016. doi: 10.1093/eurheartj/ehw211. Forthcoming.

427. Tjeerdsma G, Szabó BM, van Wijk LM, Brouwer J, Tio RA, Crijns HJ, van Veldhuisen $D$ J. Autonomic dysfunction in patients with mild heart failure and coronary artery disease and the effects of add-on beta-blockade. Eur J Heart Fail 2001; 3:33-39.

428. Fan H, Yu W, Zhang Q, Cao H, Li J, Wang J, Shao Y, Hu X. Depression after heart failure and risk of cardiovascular and all-cause mortality: a meta-analysis. Prev Med 2014;63:36-42.

429. Diez-Quevedo C, Lupón J, González B, Urrutia A, Cano L, Cabanes R, Altimir S, Coll R, Pascual T, de Antonio M, Bayes-Genis A. Depression, antidepressants, and long-term mortality in heart failure. Int J Cardiol 2013;167:1217-1225.

430. Newhouse A, Jiang W. Heart failure and depression. Heart Fail Clin 2014;10: 295-304.

431. Lahlou-Laforêt K, Ledru F, Niarra R, Consoli SM. Validity of beck depression inventory for the assessment of depressive mood in chronic heart failure Patients. J Affect Disord 2015;184:256-260.

432. Ski CF, Thompson DR, Hare DL, Stewart AG, Watson R. Cardiac Depression Scale: Mokken scaling in heart failure patients. Health Qual Life Outcomes 2012; 10:141.

433. Tu R-H, Zeng Z-Y, Zhong G-Q, Wu W-F, Lu Y-j, Bo Z-D, He Y, Huang W-Q, Yao L-M. Effects of exercise training on depression in patients with heart failure: a systematic review and meta-analysis of randomized controlled trials. Eur J Heart Fail 2014;16:749-757.

434. Blumenthal JA, Babyak MA, O'Connor C, Keteyian S, Landzberg J, Howlett J, Kraus W, Gottlieb S, Blackburn G, Swank A, Whellan DJ. Effects of exercise training on depressive symptoms in patients with chronic heart failure: the HF-ACTION randomized trial. JAMA 2012;308:465-474.

435. O'Connor CM, Jiang W, Kuchibhatla M, Silva SG, Cuffe MS, Callwood DD, Zakhary B, Stough WG, Arias RM, Rivelli SK, Krishnan R. Safety and efficacy of 
sertraline for depression in patients with heart failure: results of the SADHART-CHF (Sertraline Against Depression and Heart Disease in Chronic Heart Failure) trial. J Am Coll Cardiol 2010;56:692-699.

436. Bakris GL, Fonseca V, Katholi RE, McGill JB, Messerli FH, Phillips RA, Raskin P, Wright JT, Oakes R, Lukas MA, Anderson KM, Bell DSH, GEMINI Investigators for the. Metabolic effects of carvedilol vs metoprolol in patients with type 2 diabetes mellitus and hypertension: a randomized controlled trial. JAMA 2004;292: 2227-2236.

437. Aguilar D, Bozkurt B, Ramasubbu K, Deswal A. Relationship of hemoglobin A1C and mortality in heart failure patients with diabetes. J Am Coll Cardiol 2009;54: $422-428$.

438. Gerstein HC, Swedberg K, Carlsson J, McMurray JJ V, Michelson EL, Olofsson B, Pfeffer MA, Yusuf S. The hemoglobin A1c level as a progressive risk factor for cardiovascular death, hospitalization for heart failure, or death in patients with chronic heart failure: an analysis of the Candesartan in Heart failure: Assessment of Reduction in Mortality and Morbidity (CHARM) program. Arch Intern Med 2008;168:1699-1704.

439. Goode KM, John J, Rigby AS, Kilpatrick ES, Atkin SL, Bragadeesh T, Clark AL, Cleland JGF. Elevated glycated haemoglobin is a strong predictor of mortality in patients with left ventricular systolic dysfunction who are not receiving treatment for diabetes mellitus. Heart 2009;95:917-923.

440. MacDonald MR, Eurich DT, Majumdar SR, Lewsey JD, Bhagra S, Jhund PS, Petrie MC, McMurray JJ V, Petrie JR, McAlister FA. Treatment of type 2 diabetes and outcomes in patients with heart failure: a nested case-control study from the U.K. General Practice Research Database. Diabetes Care 2010;33:1213-1218.

441. Boussageon R, Supper I, Bejan-Angoulvant T, Kellou N, Cucherat M, Boissel J-P, Kassai B, Moreau A, Gueyffier F, Cornu C. Reappraisal of metformin efficacy in the treatment of type 2 diabetes: a meta-analysis of randomised controlled trials. PLoS Med 2012;9:e1001204.

442. Monami M, Dicembrini I, Mannucci E. Dipeptidyl peptidase-4 inhibitors and heart failure: a meta-analysis of randomized clinical trials. Nutr Metab Cardiovasc Dis 2014;24:689-697.

443. Savarese G, Perrone-Filardi P, D'Amore C, Vitale C, Trimarco B, Pani L, Rosano GMC. Cardiovascular effects of dipeptidyl peptidase-4 inhibitors in diabetic patients: a meta-analysis. Int J Cardiol 2015;181:239-244.

444. Giagulli VA, Moghetti P, Kaufman JM, Guastamacchia E, lacoviello M, Triggiani V. Managing erectile dysfunction in heart failure. Endocr Metab Immune Disord Drug Targets 2013;13:125-134.

445. Vlachopoulos C, Jackson G, Stefanadis C, Montorsi P. Erectile dysfunction in the cardiovascular patient. Eur Heart J 2013;34:2034-2046.

446. Guazzi M, Vicenzi M, Arena R, Guazzi MD. PDE5 inhibition with sildenafil improves left ventricular diastolic function, cardiac geometry, and clinical status in patients with stable systolic heart failure: results of a 1-year, prospective, randomized, placebo-controlled study. Circ Heart Fail 2011;4:8-17.

447. Giannetta E, Feola T, Gianfrilli D, Pofi R, Dall'Armi V, Badagliacca R, Barbagallo F, Lenzi A, Isidori AM, Dall'Armi V, Badagliacca R, Barbagallo F, Lenzi A, Isidori AM. Is chronic inhibition of phosphodiesterase type 5 cardioprotective and safe? A meta-analysis of randomized controlled trials. BMC Med 2014;12:185.

448. Anker SD. Uric acid and survival in chronic heart failure: validation and application in metabolic, functional, and hemodynamic staging. Circulation 2003;107: 1991-1997.

449. Zhang W. EULAR evidence-based recommendations for gout. Part II: management. Report of a task force of the EULAR Standing Committee for International Clinical Studies Including Therapeutics (ESCISIT). Ann Rheum Dis 2006;65: 1312-1324.

450. Uric acid and xanthine oxidase in heart failure - Emerging data and therapeutic implications. Doehner W, Jankowska EA, Springer J, Lainscak M, Anker SD. Int J Cardiol. 2015 Aug 11. pii: S0167-5273(15)30319-3. doi: 10.1016/j.ijcard. 2015.08.089. [Epub ahead of print]

451. Desai AS. Hyperkalemia in patients with heart failure: incidence, prevalence, and management. Curr Heart Fail Rep 2009;6:272-280.

452. Mahoney BA, Smith WA, Lo D, Tsoi K, Tonelli M, Clase C. Emergency interventions for hyperkalaemia. Cochrane Database Syst Rev 2005;2:CD003235.

453. Packham DK, Rasmussen HS, Lavin PT, El-Shahawy MA, Roger SD, Block G, Qunibi W, Pergola P, Singh B. Sodium zirconium cyclosilicate in hyperkalemia. N Engl J Med 2015;372:222-231.

454. Weir MR, Bakris GL, Bushinsky DA, Mayo MR, Garza D, Stasiv Y, Wittes J, Christ-Schmidt H, Berman L, Pitt B. Patiromer in patients with kidney disease and hyperkalemia receiving RAAS inhibitors. N Engl J Med 2015;372:211-221.

455. Anker SD, Kosiborod M, Zannad F, Piña IL, McCullough PA, Filippatos G, van der Meer P, Ponikowski P, Rasmussen HS, Lavin PT, Singh B, Yang A, Deedwania P. Maintenance of serum potassium with sodium zirconium cyclosilicate (ZS-9) in heart failure patients: results from a phase 3 randomized, double-blind, placebocontrolled trial. Eur J Heart Fail 2015;17:1050-1056.
456. Pitt B, Bakris GL, Bushinsky DA, Garza D, Mayo MR, Stasiv Y, Christ-Schmidt H, Berman L, Weir MR. Effect of patiromer on reducing serum potassium and preventing recurrent hyperkalaemia in patients with heart failure and chronic kidney disease on RAAS inhibitors. Eur J Heart Fail 2015;17:1057-1065.

457. Tavazzi L, Maggioni AP, Marchioli R, Barlera S, Franzosi MG, Latini R, Lucci D, Nicolosi GL, Porcu M, Tognoni G. Effect of rosuvastatin in patients with chronic heart failure (the GISSI-HF trial): a randomised, double-blind, placebo-controlled trial. Lancet 2008;372:1231-1239.

458. Major cardiovascular events in hypertensive patients randomized to doxazosin vs chlorthalidone. ALLHAT Collaborative Research Group. JAMA 2000;283: 1967-1975.

459. Lip GYH, Skjøth F, Overvad K, Rasmussen LH, Larsen TB. Blood pressure and prognosis in patients with incident heart failure: the Diet, Cancer and Health (DCH) cohort study. Clin Res Cardiol 2015;104:1088-1096.

460. Cohn JN, Pfeffer MA, Rouleau J, Sharpe N, Swedberg K, Straub M, Wiltse C, Wright TJ, MOXCON Investigators. Adverse mortality effect of central sympathetic inhibition with sustained-release moxonidine in patients with heart failure (MOXCON). Eur J Heart Fail 2003;5:659-667.

461. Maggioni AP, Anand I, Gottlieb SO, Latini R, Tognoni G, Cohn JN. Effects of valsartan on morbidity and mortality in patients with heart failure not receiving angiotensin-converting enzyme inhibitors. J Am Coll Cardiol 2002;40:1414-1421.

462. Pitt B, Anker SD, Bushinsky DA, Kitzman DW, Zannad F, Huang I-Z. Evaluation of the efficacy and safety of RLY5016, a polymeric potassium binder, in a doubleblind, placebo-controlled study in patients with chronic heart failure (the PEARL-HF) trial. Eur Heart J 2011;32:820-828.

463. Packer M, Bristow MR, Cohn JN, Colucci WS, Fowler MB, Gilbert EM, Shusterman NH, U.S. Carvedilol Heart Failure Study Group. The effect of carvedilol on morbidity and mortality in patients with chronic heart failure. N Engl J Med 1996:334:1349-1355.

464. Dorszewski A, Göhmann E, Dorsźewski B, Werner GS, Kreuzer H, Figulla HR. Vasodilation by urapidil in the treatment of chronic congestive heart failure in addition to angiotensin-converting enzyme inhibitors is not beneficial: results of a placebo-controlled, double-blind study. J Card Fail 1997;3:91-96.

465. Bayliss J, Norell MS, Canepa-Anson R, Reid C, Poole-Wilson P, Sutton G. Clinical importance of the renin-angiotensin system in chronic heart failure: double blind comparison of captopril and prazosin. BrMedJ (Clin Res Ed) 1985;290:1861-1865.

466. Jankowska EA, von Haehling S, Anker SD, Macdougall IC, Ponikowski P. Iron deficiency and heart failure: diagnostic dilemmas and therapeutic perspectives. Eur Heart J 2013;34:816-826.

467. Jankowska EA, Kasztura M, Sokolski M, Bronisz M, Nawrocka S, Oleśkowska-Florek W, Zymliński R, Biegus J, Siwołowski P, Banasiak W, Anker SD, Filippatos G, Cleland JGF, Ponikowski P. Iron deficiency defined as depleted iron stores accompanied by unmet cellular iron requirements identifies patients at the highest risk of death after an episode of acute heart failure. Eur Heart 2014:35:2468-2476.

468. Jankowska EA, Malyszko J, Ardehali H, Koc-Zorawska E, Banasiak W, von Haehling S, Macdougall IC, Weiss G, McMurray JJV, Anker SD, Gheorghiade M, Ponikowski P. Iron status in patients with chronic heart failure. Eur Heart J 2013;34:827-834.

469. Anker SD, Comin Colet J, Filippatos G, Willenheimer R, Dickstein K, Drexler H, Luscher TF, Bart B, Banasiak W, Niegowska J, Kirwan BA, Mori C, von Eisenhart Rothe B, Pocock SJ, Poole-Wilson PA, Ponikowski P. Ferric carboxymaltose in patients with heart failure and iron deficiency. N Engl J Med 2009;361:2436-2448.

470. Ponikowski P, van Veldhuisen DJ, Comin-Colet J, Ertl G, Komajda M, Mareev V, McDonagh T, Parkhomenko A, Tavazzi L, Levesque V, Mori C, Roubert B, Filippatos G, Ruschitzka F, Anker SD. Beneficial effects of long-term intravenous iron therapy with ferric carboxymaltose in patients with symptomatic heart failure and iron deficiency. Eur Heart J 2015;36:657-668.

471. Filippatos G, Farmakis D, Colet JC, Dickstein K, Lüscher TF, Willenheimer R, Parissis J, Gaudesius G, Mori C, von Eisenhart Rothe B, Greenlaw N, Ford I, Ponikowski P, Anker SD. Intravenous ferric carboxymaltose in iron-deficient chronic heart failure patients with and without anaemia: a subanalysis of the FAIR-HF trial. Eur J Heart Fail 2013;15:1267-1276.

472. Effects of intravenous iron therapy in iron-deficient patients with systolic heart failure: a meta-analysis of randomized controlled trials. Jankowska EA, Tkaczyszyn M, Suchocki T, Drozd M, von Haehling S, Doehner W, Banasiak W, Filippatos G, Anker SD, Ponikowski P. Eur J Heart Fail. 2016 Jan 28. doi: 10.1002/ejhf.473. [Epub ahead of print].

473. Angermann C, Pia M, Erdmann E, Levy P, Simonds AK, Somers VK, Zannad F, Cowie MR, Woehrle H, Wegscheider K, Angermann C, D'Ortho M-P, Erdmann E, Levy P, Simonds AK, Somers VK, Zannad F, Teschler H. Adaptive servo-ventilation for central sleep apnea in systolic heart failure. N Engl J Med 2015;373:1095-1105.

474. O'Meara E, Rouleau JL, White M, Roy K, Blondeau L, Ducharme A, Neagoe P-E, Sirois MG, Lavoie J, Racine N, Liszkowski M, Madore F, Tardif J-C, de Denus S, 
ANCHOR Investigators. Heart failure with anemia: novel findings on the roles of renal disease, interleukins, and specific left ventricular remodeling processes. Circ Heart Fail 2014;7:773-781.

475. Swedberg K, Young JB, Anand IS, Cheng S, Desai AS, Diaz R, Maggioni AP, McMurray JJV, O'Connor C, Pfeffer MA, Solomon SD, Sun Y, Tendera M, van Veldhuisen DJ. Treatment of anemia with darbepoetin alfa in systolic heart failure. N Engl J Med 2013;368:1210-1219.

476. Damman K, Valente MAE, Voors AA, O'Connor CM, van Veldhuisen DJ, Hillege HL. Renal impairment, worsening renal function, and outcome in patients with heart failure: an updated meta-analysis. Eur Heart J 2014;35:455-469.

477. Filippatos G, Farmakis D, Parissis J. Renal dysfunction and heart failure: things are seldom what they seem. Eur Heart / 2014;35:416-418.

478. Chawla LS, Eggers PW, Star RA, Kimmel PL. Acute kidney injury and chronic kidney disease as interconnected syndromes. N Engl / Med 2014;371:58-66.

479. Damman K, Testani JM. The kidney in heart failure: an update. Eur Heart J 2015;36: 1437-1444.

480. Clark H, Krum H, Hopper I. Worsening renal function during renin-angiotensin-aldosterone system inhibitor initiation and long-term outcomes in patients with left ventricular systolic dysfunction. Eur J Heart Fail 2014; 16:41-48.

481. Brenner S, Güder G, Berliner D, Deubner N, Fröhlich K, Ertl G, Jany B, Angermann CE, Störk S. Airway obstruction in systolic heart failure - COPD or congestion? Int J Cardiol 2013;168:1910-1916.

482. Güder G, Brenner S, Störk S, Hoes A, Rutten FH. Chronic obstructive pulmonary disease in heart failure: accurate diagnosis and treatment. Eur J Heart Fail 2014;16: $1-10$.

483. Perk J, De Backer G, Gohlke H, Graham I, Reiner Z, Verschuren M, Albus C, Benlian P, Boysen G, Cifkova R, Deaton C, Ebrahim S, Fisher M, Germano G, Hobbs R, Hoes A, Karadeniz S, Mezzani A, Prescott E, Ryden L, Scherer M, Syvänne M, Scholte op, Reimer WJM, Vrints C, Wood D, Zamorano JL, Zannad F, European Association for Cardiovascular Prevention \& Rehabilitation (EACPR), ESC Committee for Practice Guidelines (CPG). European Guidelines on cardiovascular disease prevention in clinical practice (version 2012). The Fifth Joint Task Force of the European Society of Cardiology and Other Societies on Cardiovascular Disease Prevention in Clinical Practice. Eur Heart J 2012;33: 1635-1701.

484. McKelvie RS, Moe GW, Cheung A, Costigan J, Ducharme A, Estrella-Holder E, Ezekowitz JA, Floras J, Giannetti N, Grzeslo A, Harkness K, Heckman GA, Howlett JG, Kouz S, Leblanc K, Mann E, O'Meara E, Rajda M, Rao V, Simon J, Swiggum E, Zieroth S, Arnold JMO, Ashton T, D'Astous M, Dorian P, Haddad H, Isaac DL, Leblanc M-H, Liu P, Sussex B, Ross HJ. The 2011 Canadian Cardiovascular Society heart failure management guidelines update: focus on sleep apnea, renal dysfunction, mechanical circulatory support, and palliative care. Can J Cardiol 2011;27:319-338.

485. Khayat R, Jarjoura D, Porter K, Sow A, Wannemacher J, Dohar R, Pleister A, Abraham WT. Sleep disordered breathing and post-discharge mortality in patients with acute heart failure. Eur Heart J 2015;36:1463-1469.

486. Nakamura S, Asai K, Kubota Y, Murai K, Takano H, Tsukada YT, Shimizu W. Impact of sleep-disordered breathing and efficacy of positive airway pressure on mortality in patients with chronic heart failure and sleep-disordered breathing: a meta-analysis. Clin Res Cardiol 2015;104:208-216.

487. Gottlieb DJ, Yenokyan G, Newman AB, O'Connor GT, Punjabi NM, Quan SF, Redline S, Resnick HE, Tong EK, Diener-West M, Shahar E. Prospective study of obstructive sleep apnea and incident coronary heart disease and heart failure: the Sleep Heart Health Study. Circulation 2010;122:352-360.

488. Imadojemu VA, Sinoway LI, Leuenberger UA. Vascular dysfunction in sleep apnea. Am J Respir Crit Care Med 2004;169:328-329.

489. Randerath WJ, Nothofer G, Priegnitz C, Anduleit N, Treml M, Kehl V, Galetke W. Long-term auto-servoventilation or constant positive pressure in heart failure and coexisting central with obstructive sleep apnea. Chest 2012;142:440-447.

490. Yumino D, Kasai T, Kimmerly D, Amirthalingam V, Floras JS, Bradley TD. Differing effects of obstructive and central sleep apneas on stroke volume in patients with heart failure. Am / Respir Crit Care Med 2013;187:433-438.

491. Bradley TD, Logan AG, Kimoff RJ, Series F, Morrison D, Ferguson K, Belenkie I, Pfeifer M, Fleetham J, Hanly P, Smilovitch M, Tomlinson G, Floras JS. Continuous positive airway pressure for central sleep apnea and heart failure. N Engl J Med 2005;353:2025-2033.

492. Costanzo MR, Augostini R, Goldberg LR, Ponikowski P, Stellbrink C, Javaheri S. Design of the remede System Pivotal Trial: a prospective, randomized study in the use of respiratory rhythm management to treat central sleep apnea. J Card Fail 2015;21:892-902

493. Vahanian A, Alfieri $O$, Andreotti F, Antunes MJ, Baron-Esquivias G, Baumgartner H, Borger MA, Carrel TP, De Bonis M, Evangelista A, Falk V, lung B, Lancellotti P, Pierard L, Price S, Schafers H-J, Schuler G, Stepinska J, Swedberg K, Takkenberg J, Von Oppell UO, Windecker S, Zamorano JL,
Zembala M, Bax J, Baumgartner H, Ceconi C, Dean V, Deaton C, Fagard R, Funck-Brentano C, Hasdai D, Hoes A, Kirchhof P, Knuuti J, Kolh P, McDonagh T, Moulin C, Popescu BA, Reiner Z, Sechtem U, Sirnes PA, Tendera M, Torbicki A, Vahanian A, Windecker S, Popescu BA, Von Segesser L, Badano LP, Bunc M, Claeys MJ, Drinkovic N, Filippatos G, Habib G, Kappetein AP, Kassab R, Lip GYH, Moat N, Nickenig G, Otto CM, Pepper J, Piazza N, Pieper PG, Rosenhek R, Shuka N, Schwammenthal E, Schwitter J, Mas PT, Trindade PT, Walther T. Guidelines on the management of valvular heart disease (version 2012): the Joint Task Force on the Management of Valvular Heart Disease of the European Society of Cardiology (ESC) and the European Association for Cardio-Thoracic Surgery (EACTS). Eur Heart J 2012;33:2451-2496.

494. Nishimura RA, Otto CM, Bonow RO, Carabello BA, Erwin JP, Guyton RA O'Gara PT, Ruiz CE, Skubas NJ, Sorajja P, Sundt TM, Thomas JD, American College of Cardiology/American Heart Association Task Force on Practice Guidelines. 2014 AHA/ACC guideline for the management of patients with valvular heart disease: executive summary: a report of the American College of Cardiology/American Heart Association Task Force on Practice Guidelines. J Am Coll Cardiol 2014;63:2438-2488.

495. Smith CR, Leon MB, Mack MJ, Miller DC, Moses JW, Svensson LG, Tuzcu EM, Webb JG, Fontana GP, Makkar RR, Williams M, Dewey T, Kapadia S, Babaliaros V, Thourani VH, Corso P, Pichard AD, Bavaria JE, Herrmann HC, Akin Jj, Anderson WN, Wang D, Pocock SJ. Transcatheter versus surgical aorticvalve replacement in high-risk patients. N Engl J Med 2011;364:2187-2198.

496. Leon MB, Smith CR, Mack M, Miller DC, Moses JW, Svensson LG, Tuzcu EM, Webb JG, Fontana GP, Makkar RR, Brown DL, Block PC, Guyton RA, Pichard AD, Bavaria JE, Herrmann HC, Douglas PS, Petersen JL, Akin JJ, Anderson WN, Wang D, Pocock S. Transcatheter aortic-valve implantation for aortic stenosis in patients who cannot undergo surgery. N Engl J Med 2010;363: 1597-1607.

497. Reardon MJ, Adams DH, Kleiman NS, Yakubov SJ, Coselli JS, Deeb GM, Gleason TG, Lee JS, Hermiller JB, Chetcuti S, Heiser J, Merhi W, Zorn GL, Tadros P, Robinson N, Petrossian G, Hughes GC, Harrison JK, Maini B, Mumtaz M, Conte JV, Resar JR, Aharonian V, Pfeffer T, Oh JK, Qiao H, Popma JJ. 2-Year outcomes in patients undergoing surgical or self-expanding transcatheter aortic valve replacement. J Am Coll Cardiol 2015;66:113-121.

498. Adams DH, Popma JJ, Reardon MJ, Yakubov SJ, Coselli JS, Deeb GM, Gleason TG Buchbinder M, Hermiller J, Kleiman NS, Chetcuti S, Heiser J, Merhi W, Zorn G, Tadros P, Robinson N, Petrossian G, Hughes GC, Harrison JK, Conte J, Maini B, Mumtaz M, Chenoweth S, Oh JK. Transcatheter aortic-valve replacement with a self-expanding prosthesis. N Engl J Med 2014;370:1790-1798.

499. Lancellotti P, Tribouilloy C, Hagendorff A, Popescu BA, Edvardsen T, Pierard LA Badano L, Zamorano JL. Recommendations for the echocardiographic assessment of native valvular regurgitation: an executive summary from the European Association of Cardiovascular Imaging. Eur Heart J Cardiovasc Imaging 2013;14: 611-644.

500. Chaliki HP, Mohty D, Avierinos JF, Scott CG, Schaff H V, Tajik AJ, Enriquez-Sarano M. Outcomes after aortic valve replacement in patients with severe aortic regurgitation and markedly reduced left ventricular function. Circulation 2002;106:2687-2693.

501. Smith PK, Puskas JD, Ascheim DD, Voisine P, Gelijns AC, Moskowitz AJ, Hung JW, Parides MK, Ailawadi G, Perrault LP, Acker MA, Argenziano M, Thourani V, Gammie JS, Miller MA, Pagé P, Overbey JR, Bagiella E, Dagenais F, Blackstone EH, Kron IL, Goldstein DJ, Rose EA, Moquete EG, Jeffries N, Gardner TJ, O'Gara PT, Alexander JH, Michler RE. Surgical treatment of moderate ischemic mitral regurgitation. N Engl J Med 2014;371:2178-2188.

502. Acker MA, Parides MK, Perrault LP, Moskowitz AJ, Gelijns AC, Voisine P, Smith PK, Hung JW, Blackstone EH, Puskas JD, Argenziano M, Gammie JS, Mack M, Ascheim DD, Bagiella E, Moquete EG, Ferguson TB, Horvath KA, Geller NL, Miller MA, Woo YJ, D'Alessandro DA, Ailawadi G, Dagenais F, Gardner TJ, O'Gara PT, Michler RE, Kron IL. Mitral-valve repair versus replacement for severe ischemic mitral regurgitation. N Engl J Med 2014;370:23-32.

503. De Bonis M, Al-Attar N, Antunes M, Borger M, Casselman F, Falk V, Folliguet T, lung B, Lancellotti P, Lentini S, Maisano F, Messika-Zeitoun D, Muneretto C, Pibarot P, Pierard L, Punjabi P, Rosenhek R, Suwalski P, Vahanian A. Surgical and interventional management of mitral valve regurgitation: a position statement from the European Society of Cardiology Working Groups on Cardiovascular Surgery and Valvular Heart Disease. Eur Heart J 2016;37:133-139.

504. D'ascenzo F, Moretti C, Marra WG, Montefusco A, Omede P, Taha S, Castagno D, Gaemperli O, Taramasso M, Frea S, Pidello S, Rudolph V, Franzen O, Braun D, Giannini C, Ince H, Perl L, Zoccai G, Marra S, D’Amico M, Maisano F, Rinaldi M, Gaita F. Meta-analysis of the usefulness of mitraclip in patients with functional mitral regurgitation. Am J Cardiol 2015;116:325-331.

505. Nickenig G, Estevez-Loureiro R, Franzen O, Tamburino C, Vanderheyden M, Lüscher TF, Moat N, Price S, Dall'Ara G, Winter R, Corti R, Grasso C, Snow TM, Jeger R, Blankenberg S, Settergren M, Tiroch K, Balzer J, 
Petronio AS, Büttner H-J, Ettori F, Sievert H, Fiorino MG, Claeys M, Ussia GP, Baumgartner H, Scandura S, Alamgir F, Keshavarzi F, Colombo A, Maisano F, Ebelt H, Aruta P, Lubos E, Plicht B, Schueler R, Pighi M, Di Mario C, Transcatheter Valve Treatment Sentinel Registry Investigators of the EURObservational Research Programme of the European Society of Cardiology. Percutaneous mitral valve edge-to-edge repair: in-hospital results and 1-year follow-up of 628 patients of the 2011-2012 pilot European Sentinel Registry. J Am Coll Cardiol 2014;64: $875-884$.

506. Puls M, Lubos E, Boekstegers P, von Bardeleben RS, Ouarrak T, Butter C, Zuern CS, Bekeredjian R, Sievert H, Nickenig G, Eggebrecht H, Senges J, Schillinger W. One-year outcomes and predictors of mortality after MitraClip therapy in contemporary clinical practice: results from the German transcatheter mitral valve interventions registry. Eur Heart J 2016;37:703-12.

507. Perez-Ayuso RM, Arroyo V, Planas R, Gaya J, Bory F, Rimola A, Rivera F, Rodes J. Randomized comparative study of efficacy of furosemide versus spironolactone in nonazotemic cirrhosis with ascites. Relationship between the diuretic response and the activity of the renin-aldosterone system. Gastroenterology 1983;84: 961-968.

508. Schofer J, Bijuklic K, Tiburtius C, Hansen L, Groothuis A, Hahn RT. First-in-human transcatheter tricuspid valve repair in a patient with severely regurgitant tricuspid valve. J Am Coll Cardiol 2015;65:1190-1195.

509. Herrmann HC, Pibarot P, Hueter I, Gertz ZM, Stewart WJ, Kapadia S, Tuzcu EM, Babaliaros V, Thourani V, Szeto WY, Bavaria JE, Kodali S, Hahn RT, Williams M, Miller DC, Douglas PS, Leon MB. Predictors of mortality and outcomes of therapy in low-flow severe aortic stenosis: a Placement of Aortic Transcatheter Valves (PARTNER) trial analysis. Circulation 2013;127:2316-2326.

510. Ponikowski P, Jankowska EA. Pathogenesis and clinical presentation of acute heart failure. Rev Esp Cardiol (Engl Ed) 2015;68:331-337.

511. Metra M, Felker GM, Zacà V, Bugatti S, Lombardi C, Bettari L, Voors AA, Gheorghiade M, Dei Cas L. Acute heart failure: multiple clinical profiles and mechanisms require tailored therapy. Int J Cardiol 2010;144:175-179.

512. Filippatos G, Zannad F. An introduction to acute heart failure syndromes: definition and classification. Heart Fail Rev 2007;12:87-90.

513. Alla F, Zannad F, Filippatos G. Epidemiology of acute heart failure syndromes. Heart Fail Rev 2007;12:91-95.

514. Nohria A, Tsang SW, Fang JC, Lewis EF, Jarcho JA, Mudge GH, Stevenson LW. Clinical assessment identifies hemodynamic profiles that predict outcomes in patients admitted with heart failure. J Am Coll Cardiol 2003;41:1797-1804.

515. Stevenson LW. Design of therapy for advanced heart failure. Eur J Heart Fail 2005; 7:323-331.

516. Maisel AS, Peacock WF, McMullin N, Jessie R, Fonarow GC, Wynne J, Mills RM. Timing of immunoreactive B-type natriuretic peptide levels and treatment delay in acute decompensated heart failure: an ADHERE (Acute Decompensated Heart Failure National Registry) analysis. J Am Coll Cardiol 2008;52:534-540.

517. Peacock WF, Emerman C, Costanzo MR, Diercks DB, Lopatin M, Fonarow GC. Early vasoactive drugs improve heart failure outcomes. Congest Heart Fail 15: $256-264$.

518. Wuerz RC, Meador SA. Effects of prehospital medications on mortality and length of stay in congestive heart failure. Ann Emerg Med 1992;21:669-674.

519. Chakko S, Woska D, Martinez H, de Marchena E, Futterman L, Kessler KM, Myerberg RJ. Clinical, radiographic, and hemodynamic correlations in chronic congestive heart failure: conflicting results may lead to inappropriate care. Am J Med 1991;90:353-359.

520. Wang CS, FitzGerald JM, Schulzer M, Mak E, Ayas NT. Does this dyspneic patient in the emergency department have congestive heart failure? JAMA 2005;294: 1944-1956.

521. Kelder JC, Cowie MR, McDonagh TA, Hardman SM, Grobbee DE, Cost B, Hoes AW. Quantifying the added value of BNP in suspected heart failure in general practice: an individual patient data meta-analysis. Heart 2011;97:959-963.

522. Gupta DK, Wang TJ. Natriuretic peptides and cardiometabolic health. Circ J 2015; 79:1647-1655.

523. Zois NE, Bartels ED, Hunter I, Kousholt BS, Olsen LH, Goetze JP. Natriuretic peptides in cardiometabolic regulation and disease. Nat Rev Cardiol 2014;11:403-412.

524. Nishikimi T, Kuwahara K. Current biochemistry, molecular biology, and clinical relevance of natriuretic peptides. J Cardiol 2011;57:131-140.

525. Felker GM, Mentz RJ, Teerlink JR, Voors AA, Pang PS, Ponikowski P, Greenberg BH, Filippatos G, Davison BA, Cotter G, Prescott MF, Hua TA, Lopez-Pintado S, Severin T, Metra M. Serial high sensitivity cardiac troponin T measurement in acute heart failure: insights from the RELAX-AHF study. Eur J Heart Fail 2015;17:1262-70.

526. Konstantinides S V, Torbicki A, Agnelli G, Danchin N, Fitzmaurice D, Galiè N, Gibbs JSR, Huisman MV, Humbert M, Kucher N, Lang I, Lankeit M, Lekakis J, Maack C, Mayer E, Meneveau N, Perrier A, Pruszczyk P, Rasmussen LH, Schindler TH, Svitil P, Vonk Noordegraaf A, Zamorano JL, Zompatori M, Task Force for the Diagnosis and Management of Acute Pulmonary Embolism of the
European Society of Cardiology (ESC). 2014 ESC Guidelines on the diagnosis and management of acute pulmonary embolism. Eur Heart J 2014;35:3033-3073.

527. Maisel A, Neath SX, Landsberg J, Mueller C, Nowak RM, Peacock WF, Ponikowski P, Möckel M, Hogan C, Wu AHB, Richards M, Clopton P, Filippatos GS, Di Somma S, Anand I, Ng LL, Daniels LB, Christenson RH, Potocki M, McCord J, Terracciano G, Hartmann O, Bergmann A, Morgenthaler NG, Anker SD. Use of procalcitonin for the diagnosis of pneumonia in patients presenting with a chief complaint of dyspnoea: results from the BACH (Biomarkers in Acute Heart Failure) trial. Eur J Heart Fail 2012;14: $278-286$.

528. van Deursen VM, Damman K, Hillege HL, van Beek AP, van Veldhuisen DJ, Voors AA. Abnormal liver function in relation to hemodynamic profile in heart failure patients. J Card Fail 2010;16:84-90.

529. Biegus J, Zymliński R, Sokolski M, Nawrocka S, Siwołowski P, Szachniewicz J, Jankowska EA, Banasiak W, Ponikowski P. Liver function tests in patients with acute heart failure. Pol Arch Med Wewne?trznej 2012;122:471-479.

530. Nikolaou M, Parissis J, Yilmaz MB, Seronde M-F, Kivikko M, Laribi S, Paugam-Burtz C, Cai D, Pohjanjousi P, Laterre P-F, Deye N, Poder P, Cohen-Solal A, Mebazaa A. Liver function abnormalities, clinical profile, and outcome in acute decompensated heart failure. Eur Heart J 2013;34:742-749.

531. Moe GW, Howlett J, Januzzi JL, Zowall H. N-terminal pro-B-type natriuretic peptide testing improves the management of patients with suspected acute heart failure: primary results of the Canadian prospective randomized multicenter IMPROVE-CHF study. Circulation 2007;115:3103-3110.

532. Maisel A, Mueller C, Nowak R, Peacock WF, Landsberg JW, Ponikowski P, Mockel M, Hogan C, Wu AHB, Richards M, Clopton P, Filippatos GS, Di_Somma S, Anand I, Ng L, Daniels LB, Neath S-X, Christenson R, Potocki M, McCord J, Terracciano G, Kremastinos D, Hartmann O, von Haehling S, Bergmann A, Morgenthaler NG, Anker SD, Di Somma S, Anand I, Ng L, Daniels LB, Neath S-X, Christenson R, Potocki M, McCord J, Terracciano G, Kremastinos D, Hartmann O, von Haehling S, Bergmann A, Morgenthaler NG, Anker SD. Mid-region pro-hormone markers for diagnosis and prognosis in acute dyspnea. J Am Coll Cardiol 2010;55:2062-2076.

533. Mueller C, Scholer A, Laule-Kilian K, Martina B, Schindler C, Buser P, Pfisterer M, Perruchoud AP. Use of B-type natriuretic peptide in the evaluation and management of acute dyspnea. N Engl J Med 2004;350:647-654.

534. Krishnaswamy P, Nowak RM, McCord J, Hollander JE, Duc P, Omland T, Storrow AB, Abraham WT, Wu AHB, Clopton P, Steg PG, Westheim A, Knudsen CW, Perez A, Kazanegra R, Herrmann HC, McCullough PA. Rapid measurement of B-type natriuretic peptide in the emergency diagnosis of heart failure. N Engl J Med 2002;347:161-167.

535. Task Force on the management of ST-segment elevation acute myocardial infarction of the European Society of Cardiology (ESC), Steg PG, James SK, Atar D, Badano LP, Blömstrom-Lundqvist C, Borger MA, Di Mario C, Dickstein K, Ducrocq G, Fernandez-Aviles F, Gershlick AH, Giannuzzi P, Halvorsen S, Huber K, Juni P, Kastrati A, Knuuti J, Lenzen MJ, Mahaffey KW, Valgimigli M, van 't Hof A, Widimsky P, Zahger D. ESC Guidelines for the management of acute myocardial infarction in patients presenting with ST-segment elevation. Eur Heart J 2012;33:2569-2619.

536. Levy P, Compton S, Welch R, Delgado G, Jennett A, Penugonda N, Dunne R, Zalenski R. Treatment of severe decompensated heart failure with high-dose intravenous nitroglycerin: a feasibility and outcome analysis. Ann Emerg Med 2007;50:144-152.

537. Cotter G, Metzkor E, Kaluski E, Faigenberg Z, Miller R, Simovitz A, Shaham O, Marghitay D, Koren M, Blatt A, Moshkovitz Y, Zaidenstein R, Golik A. Randomised trial of high-dose isosorbide dinitrate plus low-dose furosemide versus high-dose furosemide plus low-dose isosorbide dinitrate in severe pulmonary oedema. Lancet 1998;351:389-393.

538. Peterson PN, Rumsfeld JS, Liang L, Albert NM, Hernandez AF, Peterson ED, Fonarow GC, Masoudi FA. A validated risk score for in-hospital mortality in patients with heart failure from the American Heart Association get with the guidelines program. Circ Cardiovasc Qual Outcomes 2010;3:25-32.

539. Adams KF, Abraham WT, Yancy CW, Boscardin WJ. Risk stratification for inhospital mortality in acutely decompensated heart failure classification and regression tree analysis. JAMA 2015;293:572-580.

540. Mebazaa A, Yilmaz MB, Levy P, Ponikowski P, Peacock WF, Laribi S, Ristic AD, Lambrinou E, Masip J, Riley JP, McDonagh T, Mueller C, DeFilippi C, Harjola V-P, Thiele H, Piepoli MF, Metra M, Maggioni A, McMurray J, Dickstein K, Damman K, Seferovic PM, Ruschitzka F, Leite-Moreira AF, Bellou A, Anker SD, Filippatos G. Recommendations on pre-hospital and early hospital management of acute heart failure: a consensus paper from the HFA of the ESC, the European Society of Emergency Medicine and the Society of Academic Emergency Medicine. Eur J Heart Fail 2015;17:544-558. 
541. Weng C-L, Zhao Y-T, Liu Q-H, Fu C-J, Sun F, Ma Y-L, Chen Y-W, He Q-Y. Meta-analysis: noninvasive ventilation in acute cardiogenic pulmonary edema. Ann Intern Med 2010;152:590-600

542. Gray AJ, Goodacre S, Newby DE, Masson MA, Sampson F, Dixon S, Crane S, Elliott M, Nicholl J. A multicentre randomised controlled trial of the use of continuous positive airway pressure and non-invasive positive pressure ventilation in the early treatment of patients presenting to the emergency department with severe acute cardiogenic pulmonary oedema: the 3CPO Trial. Health Technol Assess 2009;13:1-106.

543. Vital FMR, Ladeira MT, Atallah AN. Non-invasive positive pressure ventilation (CPAP or bilevel NPPV) for cardiogenic pulmonary oedema. Cochrane Database Syst Rev 2013;5:CD005351.

544. Park M, Sangean MC, Volpe MDS, Feltrim MIZ, Nozawa E, Leite PF, Passos Amato MB, Lorenzi-Filho G. Randomized, prospective trial of oxygen, continuous positive airway pressure, and bilevel positive airway pressure by face mask in acute cardiogenic pulmonary edema. Crit Care Med 2004;32:2407-2415.

545. Gray A, Goodacre S, Newby DE, Masson M, Sampson F, Nicholl J, 3CPO Trialists. Noninvasive ventilation in acute cardiogenic pulmonary edema. N EnglJ Med 2008; 359:142-151.

546. Rawles JM, Kenmure AC. Controlled trial of oxygen in uncomplicated myocardial infarction. Br Med J 1976;1:1121-1123.

547. Park JH, Balmain S, Berry C, Morton JJ, McMurray JJV. Potentially detrimental cardiovascular effects of oxygen in patients with chronic left ventricular systolic dysfunction. Heart 2010;96:533-538.

548. Felker GM, Lee KL, Bull DA, Redfield MM, Stevenson LW, Goldsmith SR, LeWinter MM, Deswal A, Rouleau JL, Ofili EO, Anstrom KJ, Hernandez AF, McNulty SE, Velazquez EJ, Kfoury AG, Chen HH, Givertz MM, Semigran MJ, Bart BA, Mascette AM, Braunwald E, O'Connor CM. Diuretic strategies in patients with acute decompensated heart failure. N Engl J Med 2011;364:797-805.

549. Jentzer JC, DeWald TA, Hernandez AF. Combination of loop diuretics with thiazide-type diuretics in heart failure. J Am Coll Cardiol 2010;56:1527-1534.

550. O'Connor CM, Starling RC, Hernandez AF, Armstrong PW, Dickstein K, Hasselblad V, Heizer GM, Komajda M, Massie BM, McMurray IJ V, Nieminen MS, Reist CJ, Rouleau JL, Swedberg K, Adams KF, Anker SD, Atar D, Battler A, Botero R, Bohidar NR, Butler J, Clausell N, Corbalán R, Costanzo MR, Dahlstrom U, Deckelbaum LI, Diaz R, Dunlap ME, Ezekowitz JA, Feldman D, Felker GM, Fonarow GC, Gennevois D, Gottlieb SS, Hill JA, Hollander JE, Howlett JG, Hudson MP, Kociol RD, Krum H, Laucevicius A, Levy WC, Méndez GF, Metra M, Mittal S, Oh B-H, Pereira NL, Ponikowski P, Tang WHW, Wilson WH, Tanomsup S, Teerlink JR, Triposkiadis F, Troughton RW, Voors AA, Whellan DJ, Zannad F, Califf RM. Effect of nesiritide in patients with acute decompensated heart failure. N Engl J Med 2011;365: 32-43.

551. Wakai A, McCabe A, Kidney R, Brooks SC, Seupaul RA, Diercks DB, Salter N, Fermann GJ, Pospisil C. Nitrates for acute heart failure syndromes. Cochrane Database Syst Rev 2013;8:CD005151.

552. Mebazaa A, Parissis J, Porcher R, Gayat E, Nikolaou M, Boas FV, Delgado JF, Follath $F$. Short-term survival by treatment among patients hospitalized with acute heart failure: the global ALARM-HF registry using propensity scoring methods. Intensive Care Med 2011;37:290-301.

553. Sharon A, Shpirer I, Kaluski E, Moshkovitz Y, Milovanov O, Polak R, Blatt A, Simovitz A, Shaham O, Faigenberg Z, Metzger M, Stav D, Yogev R, Golik A, Krakover R, Vered Z, Cotter G. High-dose intravenous isosorbide-dinitrate is safer and better than Bi-PAP ventilation combined with conventional treatment for severe pulmonary edema. J Am Coll Cardiol 2000;36:832-837.

554. Publication Committee for the VMAC Investigators (Vasodilatation in the Management of Acute CHF). Intravenous nesiritide vs nitroglycerin for treatment of decompensated congestive heart failure: a randomized controlled trial. JAMA 2002; 287:1531-1540.

555. Cohn JN, Franciosa JA, Francis GS, Archibald D, Tristani F, Fletcher R, Montero A, Cintron G, Clarke J, Hager D, Saunders R, Cobb F, Smith R, Loeb H, Settle H. Effect of short-term infusion of sodium nitroprusside on mortality rate in acute myocardial infarction complicated by left ventricular failure: results of a Veterans Administration cooperative study. N Engl J Med 1982;306:1129-1135.

556. Elkayam U, Tasissa G, Binanay C, Stevenson LW, Gheorghiade M, Warnica JW, Young JB, Rayburn BK, Rogers JG, DeMarco T, Leier C V. Use and impact of inotropes and vasodilator therapy in hospitalized patients with severe heart failure. Am Heart J 2007;153:98-104.

557. Belletti A, Castro ML, Silvetti S, Greco T, Biondi-Zoccai G, Pasin L, Zangrillo A, Landoni $G$. The effect of inotropes and vasopressors on mortality: a meta-analysis of randomized clinical trials. BrJ Anaesth 2015;115:656-675.

558. De Backer D, Biston P, Devriendt J, Madl C, Chochrad D, Aldecoa C, Brasseur A, Defrance P, Gottignies P, Vincent J-L. Comparison of dopamine and norepinephrine in the treatment of shock. N Engl J Med 2010;362:779-789.
559. Gong B, Li Z, Yat Wong PC. Levosimendan treatment for heart failure: a systematic review and meta-analysis. J Cardiothorac Vasc Anesth 2015;29:1415-25.

560. Tang X, Liu P, Li R, Jing Q, Lv J, Liu L, Liu Y. Milrinone for the treatment of acute heart failure after acute myocardial infarction: a systematic review and meta-analysis. Basic Clin Pharmacol Toxicol 2015;117:186-194.

561. Cuffe MS, Califf RM, Adams KF, Benza R, Bourge R, Colucci WS, Massie BM, O'Connor CM, Pina I, Quigg R, Silver MA, Gheorghiade M, Outcomes of a Prospective Trial of Intravenous Milrinone for Exacerbations of Chronic Heart Failure (OPTIME-CHF) Investigators. Short-term intravenous milrinone for acute exacerbation of chronic heart failure: a randomized controlled trial. JAMA 2002; 287:1541-1547.

562. O'Connor CM, Gattis WA, Uretsky BF, Adams KF, McNulty SE, Grossman SH, McKenna WJ, Zannad F, Swedberg K, Gheorghiade M, Califf RM. Continuous intravenous dobutamine is associated with an increased risk of death in patients with advanced heart failure: insights from the Flolan International Randomized Survival Trial (FIRST). Am Heart J 1999;138:78-86.

563. Wang X-C, Zhu D-M, Shan Y-X. Dobutamine therapy is associated with worse clinical outcomes compared with nesiritide therapy for acute decompensated heart failure: a systematic review and meta-analysis. Am J Cardiovasc Drugs 2015; 15:429-37.

564. Dentali F, Douketis JD, Gianni M, Lim W, Crowther M a. Meta-analysis: anticoagulant prophylaxis to prevent symptomatic venous thromboembolism in hospitalized medical patients. Ann Intern Med 2007;146:278-288.

565. Clemo H, Wood M, Gilligan D, Ellenbogen K. Intravenous amiodarone for acute heart rate control in the critically ill patient with atrial tachyarrhythmias. Am J Cardiol 1998;81:594-598.

566. Hou ZY, Chang MS, Chen CY, Tu MS, Lin SL, Chiang HT, Woosley RL. Acute treatment of recent-onset atrial fibrillation and flutter with a tailored dosing regimen of intravenous amiodarone. A randomized, digoxin-controlled study. Eur Heart J 1995; 16:521-528.

567. Delle Karth G, Geppert A, Neunteufl T, Priglinger U, Haumer M, Gschwandtner M, Siostrzonek P, Heinz G. Amiodarone versus diltiazem for rate control in critically ill patients with atrial tachyarrhythmias. Crit Care Med 2001;29:1149-1153.

568. Iakobishvili Z, Cohen E, Garty M, Behar S, Shotan A, Sandach A, Gottlieb S, Mager A, Battler A, Hasdai D. Use of intravenous morphine for acute decompensated heart failure in patients with and without acute coronary syndromes. Acute Card Care 2011;13:76-80.

569. Peacock WF, Hollander JE, Diercks DB, Lopatin M, Fonarow G, Emerman CL. Morphine and outcomes in acute decompensated heart failure: an ADHERE analysis. Emerg Med J 2008;25:205-209.

570. Cox ZL, Lenihan DJ. Loop diuretic resistance in heart failure: resistance etiologybased strategies to restoring diuretic efficacy. J Card Fail 2014;20:611-622.

571. Mentz RJ, Kjeldsen K, Rossi GP, Voors AA, Cleland JGF, Anker SD, Gheorghiade M, Fiuzat M, Rossignol P, Zannad F, Pitt B, O'Connor C, Felker GM. Decongestion in acute heart failure. Eur J Heart Fail 2014;16:471-482.

572. Mebazaa A, Nieminen MS, Filippatos GS, Cleland JG, Salon JE, Thakkar R, Padley RJ, Huang B, Cohen-Solal A. Levosimendan vs. dobutamine: outcomes for acute heart failure patients on $\beta$-blockers in SURVIVE. Eur J Heart Fail 2009; 11:304-311.

573. Pathak A, Lebrin M, Vaccaro A, Senard JM, Despas F. Pharmacology of levosimendan: inotropic, vasodilatory and cardioprotective effects. J Clin Pharm Ther 2013; 38:341-349.

574. Papp Z, Edes I, Fruhwald S, De Hert SG, Salmenpera M, Leppikangas H, Mebazaa A, Landoni G, Grossini E, Caimmi P, Morelli A, Guarracino F, Schwinger RH, Meyer S, Algotsson L, Wikstrom BG, Jorgensen K, Filippatos G, Parissis JT, Gonzalez MJ, Parkhomenko A, Yilmaz MB, Kivikko M, Pollesello P, Follath F. Levosimendan: molecular mechanisms and clinical implications: consensus of experts on the mechanisms of action of levosimendan. Int / Cardiol 2012; 159:82-87.

575. Delaney A, Bradford C, McCaffrey J, Bagshaw SM, Lee R. Levosimendan for the treatment of acute severe heart failure: a meta-analysis of randomised controlled trials. Int J Cardiol 2010;138:281-289.

576. Monsieurs KG, Nolan JP, Bossaert LL, Greif R, Maconochie IK, Nikolaou NI, Perkins GD, Soar J, Truhláŕ A, Wyllie J, Zideman DA. European Resuscitation Council Guidelines for Resuscitation 2015: Section 1. Executive summary. Resuscitation 2015;95:1-80.

577. Gheorghiade M, Konstam MA, Burnett JC, Grinfeld L, Maggioni AP, Swedberg K, Udelson JE, Zannad F, Cook T, Ouyang J, Zimmer C, Orlandi C, Efficacy of Vasopressin Antagonism in Heart Failure Outcome Study With Tolvaptan (EVEREST) Investigators. Short-term clinical effects of tolvaptan, an oral vasopressin antagonist, in patients hospitalized for heart failure: the EVEREST Clinical Status Trials. JAMA 2007:297:1332-1343.

578. Bart BA, Goldsmith SR, Lee KL, Givertz MM, O'Connor CM, Bull DA, Redfield MM, Deswal A, Rouleau JL, LeWinter MM, Ofili EO, Stevenson LW, 
Semigran MJ, Felker GM, Chen HH, Hernandez AF, Anstrom KJ, McNulty SE, Velazquez EJ, Ibarra JC, Mascette AM, Braunwald E. Ultrafiltration in decompensated heart failure with cardiorenal syndrome. N Engl J Med 2012;367: 2296-2304.

579. Costanzo MR, Guglin ME, Saltzberg MT, Jessup ML, Bart BA, Teerlink JR, Jaski BE, Fang JC, Feller ED, Haas GJ, Anderson AS, Schollmeyer MP, Sobotka PA. Ultrafiltration versus intravenous diuretics for patients hospitalized for acute decompensated heart failure. J Am Coll Cardiol 2007;49:675-683.

580. Costanzo MR, Saltzberg MT, Jessup M, Teerlink JR, Sobotka PA. Ultrafiltration is associated with fewer rehospitalizations than continuous diuretic infusion in patients with decompensated heart failure: results from UNLOAD. J Card Fail 2010;16:277-284.

581. Mullens W, Abrahams Z, Francis GS, Taylor DO, Starling RC, Tang WHW. Prompt reduction in intra-abdominal pressure following large-volume mechanical fluid removal improves renal insufficiency in refractory decompensated heart failure. J Card Fail 2008;14:508-514.

582. Fuhrmann JT, Schmeisser A, Schulze MR, Wunderlich C, Schoen SP, Rauwolf T, Weinbrenner C, Strasser RH. Levosimendan is superior to enoximone in refractory cardiogenic shock complicating acute myocardial infarction. Crit Care Med 2008:36:2257-2266.

583. Russ MA, Prondzinsky R, Christoph A, Schlitt A, Buerke U, Söffker G, Lemm H, Swyter M, Wegener N, Winkler M, Carter JM, Reith S, Werdan K, Buerke M. Hemodynamic improvement following levosimendan treatment in patients with acute myocardial infarction and cardiogenic shock. Crit Care Med 2007;35: 2732-2739.

584. Felker GM, Benza RL, Chandler AB, Leimberger JD, Cuffe MS, Califf RM, Gheorghiade M, O'Connor CM. Heart failure etiology and response tomilrinone in decompensated heart failure. J Am Coll Cardiol 2003;41:997-1003.

585. Thiele H, Zeymer U, Neumann F-J, Ferenc M, Olbrich H-G, Hausleiter J, Richardt G, Hennersdorf M, Empen K, Fuernau G, Desch S, Eitel I, Hambrecht R, Fuhrmann J, Böhm M, Ebelt H, Schneider S, Schuler G, Werdan K. Intraaortic balloon support for myocardial infarction with cardiogenic shock. N Engl J Med 2012;367:1287-1296.

586. Thiele H, Zeymer U, Neumann F-J, Ferenc M, Olbrich H-GG, Hausleiter J, de Waha A, Richardt G, Hennersdorf M, Empen K, Fuernau G, Desch S, Eitel I, Hambrecht R, Lauer B, Böhm M, Ebelt H, Schneider S, Werdan K, Schuler G, De Waha A, Richardt G, Hennersdorf M, Empen K, Fuernau G, Desch S, Eitel I, Hambrecht R, Lauer B, Böhm M, Ebelt H, Schneider S, Werdan K, Schuler G. Intra-aortic balloon counterpulsation in acute myocardial infarction complicated by cardiogenic shock (IABP-SHOCK II): final 12 month results of a randomised, open-label trial. Lancet 2013;382:1638-1645.

587. Prins KW, Neill JM, Tyler JO, Eckman PM, Duval S. Effects of beta-blocker withdrawal in acute decompensated heart failure: a systematic review and meta-analysis. JACC Heart Fail 2015;3:647-653.

588. Santaguida PL, Don-Wauchope AC, Oremus M, McKelvie R, Ali U, Hill SA, Balion C, Booth RA, Brown JA, Bustamam A, Sohel N, Raina P. BNP and NT-proBNP as prognostic markers in persons with acute decompensated heart failure: a systematic review. Heart Fail Rev 2014;19:453-470.

589. Savarese G, Musella F, D'Amore C, Vassallo E, Losco T, Gambardella F, Cecere M, Petraglia L, Pagano G, Fimiani L, Rengo G, Leosco D, Trimarco B, Perrone-Filardi P. Changes of natriuretic peptides predict hospital admissions in patients with chronic heart failure: a meta-analysis. JACC Heart Fail 2014;2: $148-158$.

590. Volpe M, Rubattu S, Burnett J. Natriuretic peptides in cardiovascular diseases: current use and perspectives. Eur Heart J 2014;35:419-425.

591. Lainscak M, Blue L, Clark AL, Dahlström U, Dickstein K, Ekman I, McDonagh T, McMurray JJ, Ryder M, Stewart S, Strömberg A, Jaarsma T. Self-care management of heart failure: practical recommendations from the Patient Care Committee of the Heart Failure Association of the European Society of Cardiology. Eur J Heart Fail 2011;13:115-126.

592. McDonagh TA, Blue L, Clark AL, Dahlström U, Ekman I, Lainscak M, McDonald K, Ryder M, Strömberg A, Jaarsma T. European Society of Cardiology Heart Failure Association Standards for delivering heart failure care. Eur J Heart Fail 2011;13: 235-241.

593. Stewart GC, Givertz MM. Mechanical circulatory support for advanced heart failure: patients and technology in evolution. Circulation 2012;125:1304-1315.

594. Schmidt M, Burrell A, Roberts L, Bailey M, Sheldrake J, Rycus PT, Hodgson C, Scheinkestel C, Cooper DJ, Thiagarajan RR, Brodie D, Pellegrino V, Pilcher D. Predicting survival after ECMO for refractory cardiogenic shock: the survival after veno-arterial-ECMO (SAVE)-score. Eur Heart J 2015;36:1-11

595. Riebandt J, Haberl T, Mahr S, Laufer G, Rajek A, Steinlechner B, Schima H, Zimpfer D. Preoperative patient optimization using extracorporeal life support improves outcomes of INTERMACS level I patients receiving a permanent ventricular assist device. Eur J Cardiothorac Surg 2014;46:486-492.
596. Cheng JM, den Uil CA, Hoeks SE, van der Ent M, Jewbali LSD, van Domburg RT, Serruys PW. Percutaneous left ventricular assist devices vs. intra-aortic balloon pump counterpulsation for treatment of cardiogenic shock: a meta-analysis of controlled trials. Eur Heart J 2009;30:2102-2108.

597. O'Neill WW, Kleiman NS, Moses J, Henriques JPS, Dixon S, Massaro J, Palacios I, Maini B, Mulukutla S, Džavík V, Popma J, Douglas PS, Ohman M. A prospective, randomized clinical trial of hemodynamic support with impella 2.5 versus intra-aortic balloon pump in patients undergoing high-risk percutaneous coronary intervention: the PROTECT II study. Circulation 2012;126:1717-1727.

598. Rahmel A, ed. Eurotransplant International Foundation Annual Report 2013. Leiden, The Netherlands: CIP-Gegevens Koninklijke Bibliotheek, 2013.

599. Trivedi JR, Cheng A, Singh R, Williams ML, Slaughter MS. Survival on the heart transplant waiting list: impact of continuous flow left ventricular assist device as bridge to transplant. Ann Thorac Surg 2014;98:830-834.

600. Miller LW, Pagani FD, Russell SD, John R, Boyle AJ, Aaronson KD, Conte J V, Naka Y, Mancini D, Delgado RM, MacGillivray TE, Farrar DJ, Frazier OH. Use of a continuous-flow device in patients awaiting heart transplantation. $N$ Engl J Med 2007;357:885-896.

601. Kirklin JK, Naftel DC, Pagani FD, Kormos RL, Stevenson LW, Blume ED, Miller MA, Baldwin J, Young JB. Sixth INTERMACS annual report: a 10,000-patient database. J Heart Lung Transplant 2014;33:555-564.

602. Westaby S. Cardiac transplant or rotary blood pump: contemporary evidence. J Thorac Cardiovasc Surg 2013;145:24-31.

603. Teuteberg JJ, Slaughter MS, Rogers JG, McGee EC, Pagani FD, Gordon R, Rame E, Acker M, Kormos RL, Salerno C, Schleeter TP, Goldstein DJ, Shin J, Starling RC, Wozniak T, Malik AS, Silvestry S, Ewald GA, Jorde UP, Naka Y, Birks E, Najarian KB, Hathaway DR, Aaronson KD. The HVAD left ventricular assist device: risk factors for neurological events and risk mitigation strategies. JACC Heart Fail 2015;3:818-828.

604. Kirklin JK, Naftel DC, Pagani FD, Kormos RL, Stevenson LW, Blume ED, Myers SL, Miller MA, Baldwin JT, Young JB. Seventh INTERMACS annual report: 15,000 patients and counting. J Heart Lung Transplant 2015;34:1495-1504.

605. Estep JD, Starling RC, Horstmanshof DA, Milano CA, Selzman CH, Shah KB, Loebe M, Moazami N, Long JW, Stehlik J, Kasirajan V, Haas DC, O'Connell JB, Boyle AJ, Farrar DJ, Rogers JG. Risk assessment and comparative effectiveness of left ventricular assist device and medical management in ambulatory heart failure patients: results from the ROADMAP study. J Am Coll Cardiol 2015;66:1747-1761.

606. Thompson JS, Matlock DD, Mcllvennan CK, Jenkins AR, Allen LA. Development of a decision aid for patients with advanced heart failure considering a destination therapy left ventricular assist device. JACC Heart Fail 2015;3:965-976.

607. Lambrinou E, Kalogirou F, Lamnisos D, Sourtzi P. Effectiveness of heart failure management programmes with nurse-led discharge planning in reducing re-admissions: a systematic review and meta-analysis. Int J Nurs Stud 2012;49:610-624.

608. Birks EJ, Tansley PD, Hardy J, George RS, Bowles CT, Burke M, Banner NR, Khaghani A, Yacoub MH. Left ventricular assist device and drug therapy for the reversal of heart failure. N Engl J Med 2006;355:1873-1884.

609. Dandel M, Knosalla C, Hetzer R. Contribution of ventricular assist devices to the recovery of failing hearts: a review and the Berlin Heart Center Experience. Eur J Heart Fail 2014;16:248-263.

610. Jorde UP, Kushwaha SS, Tatooles AJ, Naka Y, Bhat G, Long JW, Horstmanshof DA, Kormos RL, Teuteberg JJ, Slaughter MS, Birks EJ, Farrar DJ, Park SJ. Results of the destination therapy post-food and drug administration approval study with a continuous flow left ventricular assist device: a prospective study using the INTERMACS registry (Interagency Registry for Mechanically Assisted Circulatory Support). J Am Coll Cardiol 2014;63:1751-1757.

611. Khazanie P, Rogers JG. Patient selection for left ventricular assist devices. Congest Heart Fail 2011;17:227-234.

612. Rose EA, Gelijns AC, Moskowitz AJ, Heitjan DF, Stevenson LW, Dembitsky W, Long JW, Ascheim DD, Tierney AR, Levitan RG, Watson JT, Meier P, Ronan NS, Shapiro PA, Lazar RM, Miller LW, Gupta L, Frazier OH, Desvigne-Nickens $\mathrm{P}, \mathrm{Oz}$ MC, Poirier VL. Long-term use of a left ventricular assist device for end-stage heart failure. N Engl J Med 2001;345:1435-1443.

613. Slaughter MS, Rogers JG, Milano C a, Russell SD, Conte J V, Feldman D, Sun B, Tatooles AJ, Delgado RM, Long JW, Wozniak TC, Ghumman W, Farrar DJ, Frazier $\mathrm{OH}$. Advanced heart failure treated with continuous-flow left ventricular assist device. N Engl J Med 2009;361:2241-2251.

614. Banner NR, Bonser RS, Clark AL, Clark S, Cowburn PJ, Gardner RS, Kalra PR, McDonagh T, Rogers CA, Swan L, Parameshwar J, Thomas HL, Williams SG. UK guidelines for referral and assessment of adults for heart transplantation. Heart 2011;97:1520-1527.

615. Mehra M, Kobashigawa J, Starling R, Russell S, Uber P, Parameshwar J, Mohacsi P, Augustine S, Aaronson K, Barr M. Listing criteria for heart transplantation: International Society for Heart and Lung Transplantation guidelines for the care of cardiac transplant candidates-2006. J Heart Lung Transplant 2006;25:1024-1042. 
616. Mehra MR, Canter CE, Hannan MM, Semigran MJ, Uber PA, Baran DA, Danziger-Isakov L, Kirklin JK, Kirk R, Kushwaha SS, Lund LH, Potena L, Ross HJ, Taylor DO, Verschuuren EAM, Zuckermann A. The 2016 International Society for Heart Lung Transplantation listing criteria for heart transplantation: a 10-year update. J Heart Lung Transplant 2016;35:1-23.

617. McDonagh TA, Gardner RS, Lainscak M, Nielsen OW, Parissis J, Filippatos G, Anker SD. Heart failure association of the European Society of Cardiology specialist heart failure curriculum. Eur J Heart Fail 2014;16:151-162.

618. O'Connor CM, Whellan DJ, Lee KL, Keteyian SJ, Cooper LS, Ellis SJ, Leifer ES, Kraus WE, Kitzman DW, Blumenthal JA, Rendall DS, Miller NH, Fleg JL, Schulman KA, McKelvie RS, Zannad F, Piña IL, HF-ACTION Investigators. Efficacy and safety of exercise training in patients with chronic heart failure: HF-ACTION randomized controlled trial. JAMA 2009;301:1439-1450.

619. Taylor RS, Sagar VA, Davies EJ, Briscoe S, Coats AJ, Dalal H, Lough F, Rees K, Singh S. Exercise-based rehabilitation for heart failure. Cochrane Database Syst Rev 2014;4:CD003331.

620. Nolte K, Herrmann-Lingen C, Wachter R, Gelbrich G, Dungen H-D, Duvinage A, Hoischen N, Von Oehsen K, Schwarz S, Hasenfuss G, Halle M, Pieske B, Edelmann F. Effects of exercise training on different quality of life dimensions in heart failure with preserved ejection fraction: the Ex-DHF-P trial. EurJ Prev Cardiol 2015;22:582-593.

621. Ismail H, McFarlane JR, Nojoumian AH, Dieberg G, Smart NA. Clinical outcomes and cardiovascular responses to different exercise training intensities in patients with heart failure: a systematic review and meta-analysis. JACC Heart Fail 2013;1:514-522.

622. Phillips CO, Wright SM, Kern DE, Singa RM, Shepperd S, Rubin HR. Comprehensive discharge planning with postdischarge support for older patients with congestive heart failure: a meta-analysis. JAMA 2004;291:1358-1367.

623. Stewart S, Vandenbroek AJ, Pearson S, Horowitz JD. Prolonged beneficial effects of a home-based intervention on unplanned readmissions and mortality among patients with congestive heart failure. Arch Intern Med 1999;159:257-261.

624. McAlister FA, Stewart S, Ferrua S, McMurray JJ. Multidisciplinary strategies for the management of heart failure patients at high risk for admission: a systematic review of randomized trials. J Am Coll Cardiol 2004;44:810-819.

625. Feltner C, Jones CD, Cené CW, Zheng Z-J, Sueta CA, Coker-Schwimmer EJLL, Arvanitis M, Lohr KN, Middleton JC, Jonas DE. Transitional care interventions to prevent readmissions for persons with heart failure: a systematic review and meta-analysis. Ann Intern Med 2014;160:774-784.

626. Schou M, Gustafsson F, Videbaek L, Tuxen C, Keller N, Handberg J, Sejr Knudsen A, Espersen G, Markenvard J, Egstrup K, Ulriksen H, Hildebrandt PR. Extended heart failure clinic follow-up in low-risk patients: a randomized clinical trial (NorthStar). Eur Heart J 2013;34:432-442.

627. Luttik MLA, Jaarsma T, van Geel PP, Brons M, Hillege HL, Hoes AW, de Jong R, Linssen G, Lok DJA, Berge M, van Veldhuisen DJ. Long-term follow-up in optimally treated and stable heart failure patients: primary care vs. heart failure clinic. Results of the COACH-2 study. Eur J Heart Fail 2014;16:1241-1248.

628. Abraham WT, Adamson PB, Bourge RC, Aaron MF, Costanzo MR, Stevenson LW, Strickland W, Neelagaru S, Raval N, Krueger S, Weiner S, Shavelle D, Jeffries B, Yadav JS. Wireless pulmonary artery haemodynamic monitoring in chronic heart failure: a randomised controlled trial. Lancet 2011;377:658-666.

629. Abraham WT, Stevenson LW, Bourge RC, Lindenfeld JA, Bauman JG, Adamson PB. Sustained efficacy of pulmonary artery pressure to guide adjustment of chronic heart failure therapy: complete follow-up results from the CHAMPION randomised trial. Lancet 2016;387:453-461.

630. Hindricks G, Taborsky M, Glikson M, Heinrich U, Schumacher B, Katz A, Brachmann J, Lewalter T, Goette A, Block M, Kautzner J, Sack S, Husser D, Piorkowski C, Søgaard P. Implant-based multiparameter telemonitoring of patients with heart failure (IN-TIME): a randomised controlled trial. Lancet 2014; 384:583-590.

631. Mueller C, Christ M, Cowie M, Cullen L, Maisel AS, Masip J, Miro O, McMurray J, Peacock FW, Price S, DiSomma S, Bueno H, Zeymer U, Mebazaa A. European Society of Cardiology-Acute Cardiovascular Care Association position paper on acute heart failure: a call for interdisciplinary care. Eur Heart J Acute Cardiovasc Care 2015 Jun 29. pii: 2048872615593279 [Epub ahead of print].

632. Bradley EH, Sipsma H, Horwitz LI, Ndumele CD, Brewster AL, Curry LA, Krumholz HM. Hospital strategy uptake and reductions in unplanned readmission rates for patients with heart failure: a prospective study. J Gen Intern Med 2015;30: 605-611.

633. Conraads VM, Deaton C, Piotrowicz E, Santaularia N, Tierney S, Piepoli MF, Pieske B, Schmid J-P, Dickstein K, Ponikowski PP, Jaarsma T. Adherence of heart failure patients to exercise: barriers and possible solutions: a position statement of the Study Group on Exercise Training in Heart Failure of the Heart Failure Association of the European Society of Cardiology. Eur J Heart Fail 2012;14:451-458.

634. Kitzman DW, Brubaker PH, Herrington DM, Morgan TM, Stewart KP, Hundley WG, Abdelhamed A, Haykowsky MJ. Effect of endurance exercise training on endothelial function and arterial stiffness in older patients with heart failure and preserved ejection fraction: a randomized, controlled, single-blind trial. J Am Coll Cardiol 2013;62:584-592.

635. Balion C, McKelvie R, Don-Wauchope AC, Santaguida PL, Oremus M, Keshavarz H, Hill SA, Booth RA, Ali U, Brown JA, Bustamam A, Sohel N, Raina P. B-type natriuretic peptide-guided therapy: a systematic review. Heart Fail Rev 2014;19:553-564.

636. Troughton RW, Frampton CM, Brunner-La Rocca H-P, Pfisterer M, Eurlings LWM, Erntell H, Persson H, O'Connor CM, Moertl D, Karlstrom P, Dahlstrom U, Gaggin HK, Januzzi JL, Berger R, Richards AM, Pinto YM, Nicholls MG. Effect of B-type natriuretic peptide-guided treatment of chronic heart failure on total mortality and hospitalization: an individual patient meta-analysis. Eur Heart J 2014;35:1559-1567.

637. De Vecchis R, Esposito C, Di Biase G, Ariano C, Giasi A, Cioppa C. B-type natriuretic peptide-guided versus symptom-guided therapy in outpatients with chronic heart failure: a systematic review with meta-analysis. J Cardiovasc Med (Hagerstown) 2014; 15:122-134.

638. Troughton R, Michael Felker G, Januzzi JL. Natriuretic peptide-guided heart failure management. Eur Heart J 2014;35:16-24.

639. Anker SD, Koehler F, Abraham WT. Telemedicine and remote management of patients with heart failure. Lancet 2011;378:731-739.

640. Chaudhry SI, Mattera JA, Curtis JP, Spertus JA, Herrin J, Lin Z, Phillips CO Hodshon B V, Cooper LS, Krumholz HM. Telemonitoring in patients with heart failure. N Engl J Med 2010;363:2301-2309.

641. Koehler F, Winkler S, Schieber M, Sechtem U, Stangl K, Böhm M, Boll H, Kim SS, Koehler K, Lücke S, Honold M, Heinze P, Schweizer T, Braecklein M, Kirwan B-A, Gelbrich G, Anker SD. Telemedical Interventional Monitoring in Heart Failure (TIM-HF), a randomized, controlled intervention trial investigating the impact of telemedicine on mortality in ambulatory patients with heart failure: study design. Eur J Heart Fail 2010;12:1354-1362.

642. Angermann CE, Störk S, Gelbrich G, Faller H, Jahns R, Frantz S, Loeffler M, Ertl G. Mode of action and effects of standardized collaborative disease management on mortality and mobidity in patient with systolic heart failure. Circ Heart Fail 2012;5:25-35.

643. Lyng P, Persson H, Hgg-Martinell A, Hgglund E, Hagerman I, Langius-Eklf A, Rosenqvist M, Lynga P, Persson H, Hagg-Martinell A, Hagglund E, Hagerman I, Langius-Eklof $A$, Rosenqvist $M$. Weight monitoring in patients with severe heart failure (WISH). A randomized controlled trial. Eur J Heart Fail 2012;14: 438-444.

644. Boyne JJJ, Vrijhoef HJM, Crijns HJGM, DeWeerd G, Kragten J, Gorgels APM. Tailored telemonitoring in patients with heart failure: results of a multicentre randomized controlled trial. Eur J Heart Fail 2012;14:791-801.

645. Vidán MT, Sánchez E, Fernández-Avilés F, Serra-Rexach JA, Ortiz J, Bueno H. FRAIL-HF, a study to evaluate the clinical complexity of heart failure in nondependent older patients: rationale, methods and baseline characteristics. Clin Cardiol 2014;37:725-732.

646. Turner G, Clegg A. Best practice guidelines for the management of frailty: a British Geriatrics Society, Age UK and Royal College of General Practitioners report. Age Ageing 2014;43:744-748.

647. Woo J, Leung J, Morley JE. Comparison of frailty indicators based on clinical phenotype and the multiple deficit approach in predicting mortality and physical limitation. J Am Geriatr Soc 2012;60:1478-1486.

648. Fried LP, Tangen CM, Walston J, Newman AB, Hirsch C, Gottdiener J, Seeman T, Tracy R, Kop WJ, Burke G, McBurnie MA. Frailty in older adults: evidence for a phenotype. J Gerontol A Biol Sci Med Sci 2001;56:M146-M156.

649. Folstein MF, Folstein SE, McHugh PR. "Mini-mental state". A practical method for grading the cognitive state of patients for the clinician. J Psychiatr Res 1975;12: 189-198.

650. Nasreddine ZS, Phillips NA, Bedirian V, Charbonneau S, Whitehead V, Collin I, Cummings JL, Chertkow $\mathrm{H}$. The Montreal Cognitive Assessment, MoCA: a brief screening tool for mild cognitive impairment. J Am Geriatr Soc 2005;53: 695-699.

651. Cannon JA, McMurray JJ, Quinn TJ. 'Hearts and minds': association, causation and implication of cognitive impairment in heart failure. Alzheimers Res Ther 2015; 7:22.

652. Denvir MA, Murray SA, Boyd KJ. Future care planning: a first step to palliative care for all patients with advanced heart disease. Heart 2015;101:1002-1007.

653. Evangelista LS, Lombardo D, Malik S, Ballard-Hernandez J, Motie M, Liao S. Examining the effects of an outpatient palliative care consultation on symptom burden, depression, and quality of life in patients with symptomatic heart failure. J Card Fail 2012;18:894-899.

654. Jaarsma T, Beattie JM, Ryder M, Rutten FH, McDonagh T, Mohacsi P, Murray SA, Grodzicki T, Bergh I, Metra M, Ekman I, Angermann C, Leventhal M, Pitsis A, Anker SD, Gavazzi A, Ponikowski P, Dickstein K, Delacretaz E, Blue L, Strasser F, McMurray J. Palliative care in heart failure: a position statement from the palliative care workshop of the Heart Failure Association of the European Society of Cardiology. Eur J Heart Fail 2009;11:433-443. 
655. Brännström M, Boman K. Effects of person-centred and integrated chronic heart failure and palliative home care. PREFER: a randomized controlled study. Eur J Heart Fail 2014;16:1142-1151.

656. Johnson MJ, McDonagh TA, Harkness A, McKay SE, Dargie HJ. Morphine for the relief of breathlessness in patients with chronic heart failure-a pilot study. Eur J Heart Fail 2002;4:753-756.

657. Palliative Care Outcome Scale (POS). Available at http://pos-pal.org (last accessed 2 December 2015)
658. Johnson MJ, Bland JM, Davidson PM, Newton PJ, Oxberry SG, Abernethy AP, Currow DC. The relationship between two performance scales: New York Heart Association Classification and Karnofsky Performance Status Scale.J Pain Symptom Manage 2014;47:652-658

659. Lyons KD, Bakitas M, Hegel MT, Hanscom B, Hull J, Ahles TA. Reliability and validity of the Functional Assessment of Chronic Illness TherapyPalliative care (FACIT-Pal) scale. J Pain Symptom Manage 2009;37: $23-32$. 